TABLE S7. Alignment of CU and GU strains' virulence determinants required for virulence in the human challenge model of $H$. ducreyi infection.

\title{
1) $\mathrm{CpxA}(\mathrm{HD} 1470)$
}

$\begin{array}{ll}35000 \mathrm{HP} & \text { MPKFLKKIKSVRNYLAYQLFAYFGLTFAIMLAITLAIPNFDARSFSRLEKGEHEFFIQES } \\ 33921 & \text { MPKFLKKIKSVRNYLAYQLFAYFGLTFAIMLAITLAIPNFDARSFSRLEKGEHEFFIQES } \\ \text { CIP542 } & \text { MPKFLKKIKSVRNYLAYQLFAYFGLTFAIMLAITLAIPNFDARSFSRLEKGEHEFFIQES } \\ \text { DMC64 } & \text { MPKFLKKIKSVRNYLAYQLFAYFGLTFAIMLAITLAIPNFDARSFSRLEKGEHEFFIQES } \\ \text { DMC111 } & \text { MPKFLKKIKSVRNYLAYQLFAYFGLTFAIMLAITLAIPNFDARSFSRLEKGEHEFFIQES } \\ & * * * * * * * * * * * * * * * * * * * * * * * * * * * * * * * * * * * * * * * * * * * * * * * * * * * * * * * * * * * *\end{array}$

$35000 \mathrm{HP}$ RQTELQYNLDEIFERRLSVATQNGFNIILFDPKTRIFVGAGADSNRIQSFQVFLYRAQTP

33921

CIP542

DMC6 4

DMC 111 RQTELQYNLDEIFERRLSVATQSGFNI ILFDPKTRIFVGAGADSNRIQSFQVFLYRAQTP ROTELQYNLDE IFERRLSVATQSGFNI ILFDPKTRIFVGAGADSNRIOSFOVFLYRAOTP RQTELQYNLDEIFERRLSVATQSGFNI ILFDPKTRIFVGAGADSNRIQSFQVFLYRAQTP RQTELQYNLDE IFERRLSVATQSGFNIILFDPKTRIFVGAGADSNRIQSFQVFLYRAQTP $* * * * * * * * * * * * * * * * * * * * * * . * * * * * * * * * * * * * * * * * * * * * * * * * * * * * * * * * * * * *$

$35000 \mathrm{HP} \quad$ LEPLORRFGSLEISGPFLVKSNKREYMOYFAOIVDPOEEFFNRIFDSPWLMLMIVLIVSV

33921

CIP5 42 LEPLQRRFGSLEISGPFLVKSNKREYMQYFAQIVDPQEEFFNRIFDSPWLMLMIVLIVSV LEPLQRRFGSLEISGPFLVKSNKREYMQYFAQIVDPQEEFFNRIFDSPWLMLMIVLIVSV LEPLQRRFGSLEISGPFLVKSNKREYMQYFAQIVDPQEEFFNRIFDSPWLMLMIVLIVSV

DMC64

DMC 111 LEPLQRRFGSLEISGPFLVKSNKREYMQYFAQIVDPQEEFFNRIFDSPWLMLMIVLIVSV

PILLWLSWKIARPVKELRICANAVATGNLAINPKLETEGIHEFREVGRSFNOMITSLQEL PILLWLSWKIARPVKELRICANAVATGNLAINPKLETEGIHEFREVGRSFNQMITSLQEL PILLWLSWKIARPVKELRICANAVATGNLAINPKLETEGIHEFREVGRSFNQMITSLQEL PILLWLSWKIARPVKELRICANAVATGNLAINPKLETEGI HEFREVGRSFNQMITSLQEL PILLWLSWKIARPVKELRICANAVATGNLAINPKLETEGIHEFREVGRSFNQMITSLQEL $* * * * * * * * * * * * * * * * * * * * * * * * * * * * * * * * * * * * * * * * * * * * * * * * * * * * * * * * * * * *$

$35000 \mathrm{HP}$

33921

CIP5 42

DMC 64

DMC 111

$35000 \mathrm{HP}$

33921

CIP5 42

DMC 64

TEYQQRLLSDISHELKTPLARLQLATALIRRRNGDSAELTRIDNQIMKLDTMVHDLLSLS TEYQQRLLSDISHELKTPLARLQLATALIRRRNGDSAELTRIDNQIMKLDTMVHDLLSLS TEYQQRLLSDISHELKTPLARLQLATALIRRRNGDSAELTRIDNQIMKLDTMVHDLLSLS TEYQQRLLSDISHELKTPLARLQLATALIRRRNGDSAELTRIDNQIMKLDTMVHDLLSLS TEYQQRLLSDISHELKTPLARLQLATALIRRRNGDSAELTRIDNQIMKLDTMVHDLLSLS $* * * * * * * * * * * * * * * * * * * * * * * * * * * * * * * * * * * * * * * * * * * * * * * * * * * * * * * * * * * *$

DMC 111

$35000 \mathrm{HP}$

33921

CIP5 42

DMC 64

RQQINQHLMREVFSINKIWDDILEDAKFEAEQNQIDLF IEQR IDNVEGYF INGNEI ILAS RQQINQHLMREVFS INK IWDD ILEDAKFEAEQNQIDLF IEQR I DNVEGYF INGNE I ILAS RQQINQHLMREVFS INK IWDD ILEDAKFEAEQNQIDLF IEQR IDNVEGYF INGNE I ILAS RQQINQHLMREVFS INK IWDD ILEDAKFEAEQNQIDLF IEQR I DNVEGYF INGNE I ILAS RQQINQHLMREVFS INKIWDDILEDAKFEAEQNQIDLF IEQR IDNVEGYF INGNEIILAS $* * * * * * * * * * * * * * * * * * * * * * * * * * * * * * * * * * * * * * * * * * * * * * * * * * * * * * * * * * * *$

DMC 111

ALENLIRNAQKYAKQSITVLIYIDDKELVMSVDDDGEGVPESEYKQIFRPFYRVGEARDR ALENLIRNAQKYAKQSITVLIYIDDKELVMSVDDDGEGVPESEYKQIFRPFYRVGEARDR ALENLIRNAQKYAKQSITVLIYIDDKELVMSVDDDGEGVPESEYKQIFRPFYRVGEARDR ALENLIRNAQKYAKQSITVLIYIDDKELVMSVDDDGEGVPESEYKQIFRPFYRVGEARDR ALENLIRNAQKYAKQSITVLIYIDDKELVMSVDDDGEGVPESEYKQIFRPFYRVGEARDR $* * * * * * * * * * * * * * * * * * * * * * * * * * * * * * * * * * * * * * * * * * * * * * * * * * * * * * * * * * * *$

Note: The CpxA sequence of NZS1, NZS2, NZS3, NZS4, NZV1, 82-029362, 6644, HD183, HMC46, and HMC5 6 were identical to that of $35000 \mathrm{HP}$.

\section{2) CsrA (HD1430)}

The CsrA sequence of all the class I and class II strains were identical to $35000 \mathrm{HP}$. 


\section{3) DksA (HD0603)}

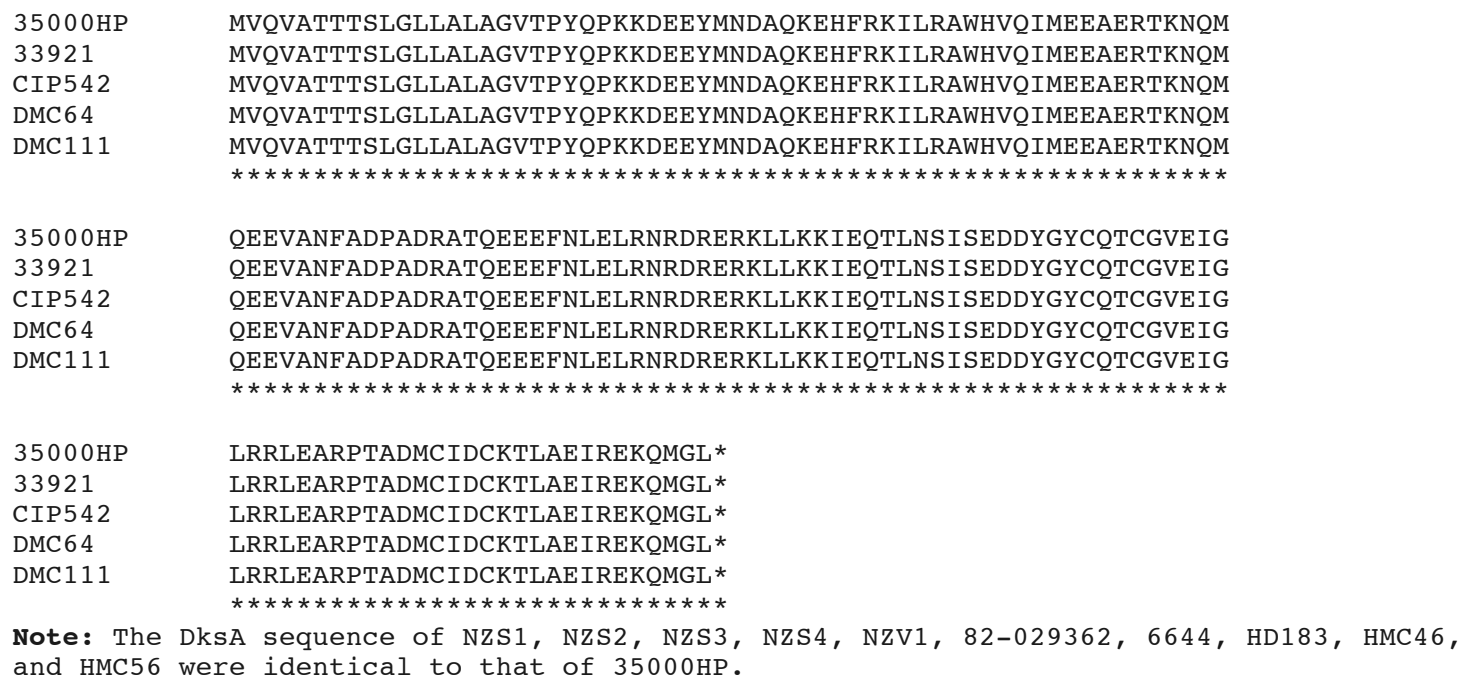

\section{4) DltA (HD0746)}

$\begin{array}{ll}35000 \mathrm{HP} & \text { MKTLGLFSIIGLLSGCVYAPPAVDHPIEDTVVIQSEHHHTIYHESDKYDRDKYDRNDRYP } \\ 33921 & \text { MKTLGLFSIIGLLSGCVYAPPAVDNPIEDTVVIQSEHHHMIYHESDKYDRDKYDRNDRYP } \\ \text { CIP542 } & \text { MKTLGLFSIIGLLSGCVYAPPAVDNPIEDTVVIQSEHHHMIYHESDKYDRDKYDRNDRYP } \\ \text { DMC64 } & \text { MKTLGLFSIIGLLSGCVYAPPAVDNPIEDTVVIQSEHHHMIYHESDKYDRDKYDRNDRYP } \\ \text { DMC111 } & \text { MKTLGLFSIIGLLSGCVYAPPAVDNPIEDTVVIQSEHHHMIYHESDKYDRDKYDRNDRYP } \\ & * * * * * * * * * * * * * * * * * * * * * * * * * * * * * * * * * * * * * * * * * * * * * * * * * * * * * * * * *\end{array}$

$35000 \mathrm{HP} \quad$ HYRYTGEIRTYWGECLDQSRSNYKGIISYRCHGGDNQRFTFYRDS IRVNGQCLDVGSENK

33921 HYHYTGEIRTYWGKCLDQSRSNYKGIISYRCHGGDNQRFTFYRDSIRVNGQCLDVGSENK

CIP5 42

DMC6 4

DMC 111 HYHYTGEIRTYWGKCLDQSRSNYKGI ISYRCHGGDNQRFTFYRDS IRVNGQCLDVGSENK HYHYTGEIRTYWGKCLDQSRSNYKGI ISYRCHGGDNQRFTFYRDS IRVNGQCLDVGSENK HYHYTGE IRTYWGKCLDQSRSNYKGI ISYRCHGGDNQRFTFYRDS IRVNGQCLDVGSENK $* *: * * * * * * * * * *: * * * * * * * * * * * * * * * * * * * * * * * * * * * * * * * * * * * * * * * * * * * * * *$

$35000 \mathrm{HP}$

33921

CIP5 42

DMC 64

DMC 111

FDGARIIAYRCHGGKNQRWFRQGHQIRSEMNGKCLEVGRDRNKLTLQQCDGSRSQOFFY* FDGARI IAYRCHGGKNQRWFRQGHQIRSEMNGKCLEVGRDRNKLTLQQCDGSRSQQFFY * FDGARI IAYRCHGGKNQRWFRQGHQIRSEMNGKCLEVGRDRNKLTLQQCDGSRSQQFFY * FDGARI IAYRCHGGKNQRWFRQGHQIRSEMNGKCLEVGRDRNKLTLQQCDGSRSQQFFY * FDGARIIAYRCHGGKNQRWFRQGHQIRSEMNGKCLEVGRDRNKLTLQQCDGSRSQQFFY * $* * * * * * * * * * * * * * * * * * * * * * * * * * * * * * * * * * * * * * * * * * * * * * * * * * * * * * * * * * * *$

Note: The DltA sequence of NZS1, NZS2, NZS3, NZS4, NZV1, 82-029362, 6644, HD183, HMC46, and HMC56 were identical to that of $35000 \mathrm{HP}$.

\section{5) DsrA (HD0769)}

$35000 \mathrm{HP}$

NZS1

NZS2

NZS 3

NZS 4

NZV1

82-029362

6644

HD 183

HMC 46

HMC 56

33921

CIP5 42

DMC 64

DMC 111
------MKIKCLVAVVGLACSTITTMAQQPPKFAGVSSLYSYEYDYGKGKWTWSNEGGFD ------MKIKCLVAVVGLACSTITTMAQQPPKFAGVSSLYSYEYDYGKGKWTWSNEDGFD -----MKIKCLVAVVGLACSTITTMAQQPPKFAGVSSLYSYEYDYGKGKWTWSNEDGFD -----MKIKCLVAVVGLACSTITTMAQQPPKFAGVSSLYSYEYDYGKGKWTWSNEDGFD ------MKIKCLVAVVGLACSTITTMAQQPPKFAGVSSLYSYEYDYGKGKWTWSNEDGFD ------MKIKCLVAVVGLACSTITTMAQQPPKFAGVSSLYSYEYDYGKGKWTWSNEDGFD -----MKIKCLVAVVGLACSTITTMAQQPPKFAGVSSLDSYEYDYGKGKWTWSEKDGFD -----MKIKCLVAVVGLACSTITTMAQQPPKFAGVSSLDSYEYDYGKGKWTWSEKDGFD ------MKIKCLVAVVGLACSTITTMAQQPPKFAGVSSLYSYEYDYGKGKWTWSNEGGFD -----MKIKCLVAVVGLACSTITTMAQQPPKFAGVSSLDSYEYDYGKGKWTWSEKDGFD ------MKIKCLVAVVGLACSTITTMAQQPPKFAGVSSLDSYEYDYGKGKWTWSEKDGFD MKMKMKMKMKLTALMVGLVASNGSLSAQMQPQNFDLGRIKIGDVSFDVDAF IKRV-DELD MKMKMKMKMKLTALMVGLVASNGSLSAQMOPQNFDLGR IKIGDVSFDVDAF IKRV-DELD MKMKMKMKMKLTALMVGLVASNGSLSAQMQPQNFDLGRIKIGDVSFDVDAF I KRV-DELD MKMKMKMKMKLTALMVGLVASNGSLSAQMQPQNFDLGR IKIGDVSFDVDAF IKRV-DELD

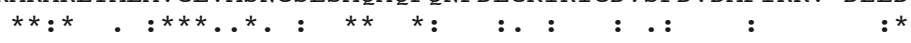


$35000 \mathrm{HP}$

NZS 1

NZS2

NZS3

NZS4

NZV1

82-029362

6644

HD 183

HMC 46

HMC 56

33921

CIP 542

DMC6 4

DMC 111

$35000 \mathrm{HP}$

NZS 1

NZS2

NZS 3

NZS4

NZV1

82-029362

6644

HD 183

HMC 46

HMC 56

33921

CIP 542

DMC6 4

DMC 111

$35000 \mathrm{HP}$

NZS 1

NZS2

NZS3

NZS4

NZV1

82-029362

6644

HD 183

HMC 46

HMC 56

33921

CIP 542

DMC 64

DMC 111

$35000 \mathrm{HP}$

NZS 1

NZS2

NZS 3

NZS4

NZV1

82-029362

6644

HD 183

HMC 46

HMC 56

33921

CIP 542

DMC 64

DMC 111

$35000 \mathrm{HP}$

NZS 1

NZS2

NZS 3

NZS 4
-IKVPGIKMKPKEWISKOATYLELQHYMPYTPVLVTYA-PGVSPSPILLYPMSDPDQLGI -IKAPRIKMKPKEWISKQVTYLKLQHYMPYTPVLVTSDVPP--PSSILLYPMSDPDQLGI -IKAPRIKMKPKEWISKQVTYLKLQHYMPYTPVLVTSDVPPPPP SS ILLYPMSDPDQLGI -IKAPRIKMKPKEWISKQVTYLKLQHYMPYTPVLVTSDVPPPPPSS ILLYPMSDPDQLGI -IKAPRIKMKPKEWISKQVTYLKLQHYMPYTPVLVTSDVPPP-PSSILLYPMSDPDQLGI -IKAPRIKMKPKEWISKQVTYLKLQHYMPYTPVLVTSDVPPP-PSSILLYPMSDPDQLGI -IKAPGIKMKPKKWISRQATYLGLQHYMPYTPVLVTYAS--AEPNTVLLYPMPDPDQLGI -IKAPGIKMKPKKWISRQATYLGLQHYMPYTPVLVTYAS--AEPNTVLLYPMPDPDQLGI -IKVPGIKMKPKEWISKQATYLELQHYMPYTPVLVTSA-PDVPPSS ILLYPMSDPDQLGI -IKAPGIKMKPKKWISRQATYLGLQHYMPYTPVLVTYAS--AEPNTVLLYPMPDPDQLGI -IKAPGIKMKPKKWISRQATYLGLQHYMPYTPVLVTYAS--AEPNTVLLYPMPDPDQLGI KNIRDGIGLGDEDFKRKYYSYVDHE---------LGS-----MIGSPFIYKEKNPLGNGI KNIRDGIGLGDEDFKRKYYSYVDHE--------LGS-----MIGSPFIYKEKNPLGNGI KNIRDGIGLGDEDFKRKYYSYVDHE---------LGS-----MIGSPF IYKEKNPLGNGI KNIRDGIGLGDEDFKRKYYSYVDHE----------LGS-----MIGSPFIYKEKNPLGNGI * : :.: : :*: : $:$ : :* :* **

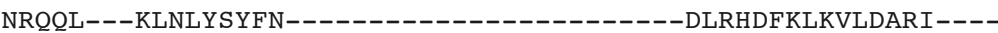
NRQQL---KLNLYSYFN------------------------DLRHDFKLNVLDARI---NRQQL---KLNLYSYFN----------------------DLRHDFKLNVLDARI---NROOL---KLNLYSYFN-_-_-_-_-_-_-_-_-_---DLRHDFKLNVLDARI-_-NRQQL---KLNLYSYFN-----------------------DLRHDFKLNVLDARI---NRQQL---KLNLYSYFN----------------------DLRHDFKLNVLDARI---NRQQL---KLNLYSYFN----------------------DLRHGF KLNVLDARI---NRQQL---KLNLYSYFN-----------------------DLRHGFKLNVLDARI---NRQQL---KLNLYSYFN-----------------------DLRHDFKLKVLDARI---NRQQL---KLNLYSYFN-----------------------DLRHGFKLNVLDARI----

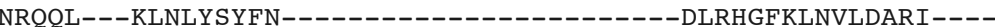
ARGLGYSGKLTMPRYIRGPLLRRSVIRGYLLGRVIGRQAHVQAKNRLNMELLQSRLMSAS ARGLGYSGKLTMPRY IRGPLLRRSVIRGYLLGRVIGRQAHVQAKNRLNMELLQSRLMSAS ARGLGYSGKLTMPRY IRGPLLRLSVIRGYLLGRVIGROAHVQAKNRLNMELLQSRLMSAS ARGLGYSGKLTMPRYIRGPLLRLSVIRGYLLGRVIGRQAHVQAKNRLNMELLQSRLMSAS

* $\quad * * .: \quad *:$. $:: .::::: *:: *$ :

SKNKONIDTISKYLLELGTYLDDSYRMMEQNTH--------------NINKLSKELQTGL SKNKONIDTISKHLLELGTYLDGSYRMMEQNTHNINKN-------THNINKLSKELQTGL SKNKQNIDTISKHLLELGTYLDGSYRMMEQNTHNINKN-------THNINKLSKELQTGL SKNKQNIDTISKHLLELGTYLDGSYRMMEQNTHNINKN-------THNINKLSKELQTGL SKNKQNIDTISKHLLELGTYLDGSYRMMEQNTHNINKN-------THNINKLSKELQTGL SKNKONIDTISKHLLELGTYLDGSYRMMEONTHNINKN--------THNINKLSKELOTGL SQNKQNIDTISEYLLKLGTYLDSSYRMMEQNTHNINKN-------THNINKLSKELQTGL SQNKONIDTISEYLLKLGTYLDSSYRMMEQNTHNINKN-------THNINKLSKELQTGL SKNKONIDTISKYLLELGTYLDGSYRMMEONTHNINKNTHNINKNTHNINKLSKELQTGL SQNKONIDTISEYLLKLGTYLDSSYRMMEQNTHNINKN-------THNINKLSKELQTGL SQNKQNIDTISEYLLKLGTYLDSSYRMMEQNTHNINKN-------THNINKLSKELQTGL LLNRAE ILNVGNHLNSLLKYTEGAFAFLN---GGVTKNSADIFKNTORIDALSKNLETGL LLNRAEILNVGNHLNSLLKYTEGAFAFLN---GGVTKNSADIFKNTQRIDALSKNLETGL LLNRAEILNVGNHLNSLLKYTEGAFAFLN---GGVTKNSADIFKNTQRIDALSKNLETGL LLNRAEILNVGNHLNSLLKYTEGAFAFLN---GGVTKNSADIFKNTQRIDALSKNLETGL

*: :* .: .::* .* .* : : : : : : .*:***:*:**

ANQSALSMLVQPNGVGKTSVSAAVGGYRDKTALAIGVGSRITDRFTAKAGVAFNTYNGGM ANQSALSMLVQPNGVGKTSVSAAVGGYRDKTALAIGVGSRITDRFTAKAGVAFNTYNGGM ANQSALSMLVQPNGVGKTSVSAAVGGYRDKTALAIGVGSRITDRFTAKAGVAFNTYNGGM ANQSALSMLVQPNGVGKTSVSAAVGGYRDKTALAIGVGSRITDRFTAKAGVAFNTYNGGM ANQSALSMLVQPNGVGKTSVSAAVGGYRDKTALAIGVGSRITDRFTAKAGVAFNTYNGGM ANQSALSMLVQPNGVGKTSVSAAVGGYRDKTALAIGVGSRITDRFTAKAGVAFNTYNGGM ANQSALSMLVQPNGVGKTSVSAAVGGYRDKTALAIGVGSRITDRFTAKAGVAFNTYNGGM ANQSALSMLVQPNGVGKTSVSAAVGGYRDKTALAIGVGSRITDRFTAKAGVAFNTYNGGM ANOSALSMLVQPNGVGKTSVSAAVGGYRDKTALAIGVGSRITDRFTAKAGVAFNTYNGGM ANQSALSMLVQPNGVGKTSVSAAVGGYRDKTALAIGVGSRITDRFTAKAGVAFNTYNGGM ANQSALSMLVQPNGVGKTSVSAAVGGYRDKTALAIGVGSRITDRFTAKAGVAFNTYNGGM ANQSALSMLVQPNGVGKTSVSAAVGGYRGKSALAIGVGSRITDAFTAKAGVAFNTYNGGM ANQSALSMLVQPNGVGKTSVSAAVGGYRGKSALAIGVGSRITDAFTAKAGVAFNTYNGGM ANQSALSMLVQPNGVGKTSVSAAVGGYRGKSALAIGVGSRITDAFTAKAGVAFNTYNGGM ANQSALSMLVQPNGVGKTSVSAAVGGYRGKSALAIGVGSRITDAFTAKAGVAFNTYNGGM $\star * * * * * * * * * * * * * * * * * * * * * * * * * * * \quad *: * * * * * * * * * * * * \quad * * * * * * * * * * * * * * * *$

SYGASVGYEF

SYGASVGYEF

SYGASVGYEF

SYGASVGYEF

SYGASVGYEF 


\begin{tabular}{|c|c|}
\hline NZV1 & SYGASVGYEF \\
\hline $82-029362$ & SYGASVGYEF \\
\hline 6644 & SYGASVGYEF \\
\hline HD 183 & SYGASVGYEF \\
\hline HMC 46 & SYGASVGYEF \\
\hline HMC 56 & SYGASVGYEF \\
\hline 33921 & SYGASVGYEF \\
\hline CIP 542 & SYGASVGYEF \\
\hline DMC6 4 & SYGASVGYEF \\
\hline DMC 111 & SYGASVGYEF \\
\hline
\end{tabular}

\section{6) $\mathrm{FgbA}(\mathrm{HD0192})$}

$35000 \mathrm{HP}$

82-029362

6644

HMC 46

HMC 56

33921

CIP 542

DMC 64

DMC 111

$35000 \mathrm{HP}$

82-029362

6644

HMC 46

HMC 56

33921

CIP 542

DMC 64

DMC 111

$35000 \mathrm{HP}$

82-029362

6644

HMC 46

HMC5 6

33921

CIP 542

DMC 64

DMC 111

MKKS ILALVLGTTFTLAACDKPQVEEMKQTVTDTAANTKAIVVEKAGDMKESASEMKDAA MKKSILALVLGTTFTLAACDKPOVEEMKOTVTDTAANTKAIVVEKAGDMKESASEMKDAA MKKSILALVLGTTFTLAACDKPQVEEMKOTVTDTAANTKAIVVEKAGDMKESASEMKDAA MKKS ILALVLGTTFTLAACDKPQVEEMKQTVTDTAANTKAIVVEKAGDMKESASEMKDAA MKKSILALVLGTTFTLAACDKPQVEEMKQTVTDTAANTKAIVVEKAGDMKESASEMKDAA MKKSILALVLGTTFTLAACDKPQVEEMKQTVTDTAANTKAIVVEKAGDMKESANEMKDAA MKKSILALVLGTTFTLAACDKPQVEEMKQTVTDTAANTKAIVVEKAGDMKESANEMKDAA MKKS ILALVLGTTFTLAACDKPQVEEMKQTVTDTAANTKAIVVEKAGDMKESANEMKDAA MKKS ILALVLGTTFTLAACDKPOVEEMKOTVTDTAANTKAIVVEKAGDMKESANEMKDAA $* * * * * * * * * * * * * * * * * * * * * * * * * * * * * * * * * * * * * * * * * * * * * * * * * * * * * * * * * * * . * * * * * *$

KAKLEDMKESAAEAKESLAEKANEMKDAAKAKLEGMKESAAEAKESLAEKANEMKDAAKA

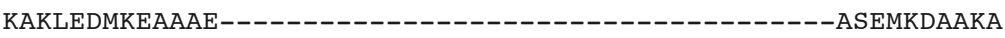

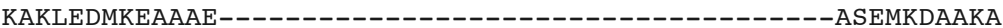

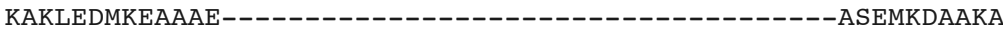

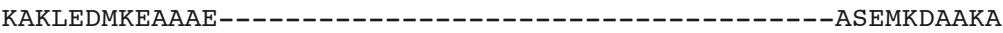

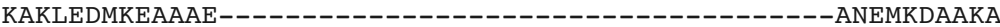
KAKLEDMKEAAAE---------------------------------ANEMKDAAKA

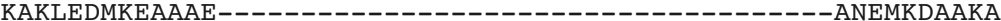
KAKLEDMKEAAAE--------------------------------ANEMKDAAKA $* * * * * * * *$ : ***) $\quad ._{*} * * * * * * *$

KLEDMKEAAADKKAEMAEKMDSAADAMKEKVDEMKK*-KLEDMKEAAADKKAEMAEKMDSAADAMKEKVDEMKK-- * KLEDMKEAAADKKAEMAEKMDSAADAMKEKVDEMKK--* KLEDMKEAAADKKAEMAEKMDSAADAMKEKVDEMKK-- * KLEDMKEAAADKKAEMAEKMDSAADAMKEKVDEMKK-- * KLEDMKEAAADKKAEMAEKKKWTPQLMO *KKKWTK * KNX KLEDMKEAAADKKAEMAEKKKWTPOLMO*KKKWTK *KNX KLEDMKEAAADKKAEMAEKKKWTPQLMQ*KKKWTK*KNX KLEDMKEAAADKKAEMAEKKKWTPOLMO *KKKWTK * KNX

$* * * * * * * * * * * * * * * * *$. : : : *: *. * *

Note: The FgbA sequence of NZS1, NZS2, NZS3, NZS4, and NZV1 were identical to that of $35000 \mathrm{HP}$.

\section{7) Flp1 (HD1312)}

$35000 \mathrm{HP}$

NZS 1

NZS2

NZS3

NZS 4

NZV1

82-029362

6644

HD 183

HMC 46

HMC5 6

33921

CIP 542

DMC64

DMC 111

$35000 \mathrm{HP}$

NZS 1

NZS2
MLSVLMTQAYISATESLRTSIQRFRKNQQGVTAIEYGLIAVAVAILI IAVFYNNQGFLMK MLSVLMTQAY ISATESLRTS IQRFRKNQQGVTA IEYGL IAVAVAIL I IAVFYNNQGFLMK MLSVLMTQAYISATESLRTSIQRFRKNQQGVTAIEYGLIAVAVAILI IAVFYNNQGFLMK MLSVLMTQAYISATESLRTSIQRFRKNQQGVTAIEYGLIAVAVAILI IAVFYNNQGFLMK MLSVLMTQAYISATESLRTS IQRFRKNQQGVTA IEYGLIAVAVAILI IAVFYNNQGFLMK MLSVLMTQAYISATESLRTSIQRFRKNQQGVTAIEYGLIAVAVAILI IAVFYNNQGFLMK MLSVLMTQAYISATESLRTSIQRFRKNQQGVTAIEYGLIAVAVAILI IAVFYNNQGFLMK MLSVLMTQAYISATESLRTS IQRFRKNQQGVTAIEYGLIAVAVAILI IAVFYNNQGFLMK MLSVLMTQAYISATESLRTSIQRFRKNQQGVTAIEYGLIAVAVAILI IAVFYNNQGFLMK MLSVLMTQAYISATESLRTSIQRFRKNQQGVTAIEYGLIAVAVAILI IAVFYNNQGFLMK MLSVLMTQAY ISATESLRTS IQRFRKNQQGVTAIEYGLIAVAVAILI IAVFYNNQGFLMK MLSTLTTQAYISATESVRAGIRRFKENQQGVTAIEYGLIAVAVAVLI IAAFYSENSFIKK MLSTLTTQAYISATESVRAGIRRFKENQQGVTAIEYGLIAVAVAVLI IAAFYSENSF IKK MLSTLTTQAYISATESVRAGIRRFKENQQGVTAIEYGLIAVAVAVLIIAAFYSENSFIKK MLSTLTTQAYISATESVRAGIRRFKENQQGVTAIEYGLIAVAVAVLI IAAFYSENSFIKK $* * * . * * * * * * * * * * *: *: . *: * *:: * * * * * * * * * * * * * * * * * *: * * * * . * * .:: .{ }^{*}: *$

LKTKFSDLATGISSANGTTSLNSFK* LKTKF SDLATGISSANGTTSLNSFK* LKTKF SDLATGISSANGTTSLNSFK* 


$\begin{array}{ll}\text { NZS3 } & \text { LKTKFSDLATGISSANGTTSLNSFK* } \\ \text { NZS4 } & \text { LKTKFSDLATGISSANGTTSLNSFK* } \\ \text { NZV1 } & \text { LKTKFSDLATGISSANGTTSLNSFK* } \\ 82-029362 & \text { LKTKFSDLATGISSANGTTSLNSFK* } \\ 6644 & \text { LKTKFSDLATGISSANGTTSLNSFK* } \\ \text { HD183 } & \text { LKTKFSDLATGISSANGTTSLNSFK* } \\ \text { HMC46 } & \text { LKTKFSDLATGISSANGTTSLNSFK* } \\ \text { HMC56 } & \text { LKTKFSDLATGISSANGTTSLNSFK* } \\ 33921 & \text { LQSKFSSLASGISNQNINFT*----- } \\ \text { CIP542 } & \text { LQSKFSSLASGISNQNINFT*----- } \\ \text { DMC64 } & \text { LQSKFSSLASGISNQNINFT*----- } \\ \text { DMC111 } & \text { LQSKFSSLASGISNQNINFT*----- } \\ & \text { *: ***.**:*** * . : }\end{array}$

\section{8) Flp2 (HD1311)}

$35000 \mathrm{HP}$

NZS 1

NZS2

NZS 3

NZS 4

NZV1

82-029362

6644

HD 183

HMC 46

HMC 56

33921

CIP 542

DMC 64

DMC 111

$35000 \mathrm{HP}$

NZS 1

NZS2

NZS 3

NZS 4

NZV1

82-029362

6644

HD 183

HMC 46

HMC 56

33921

CIP 542

DMC6 4

DMC 111
MLSVLMTQAYISATESLRTSIQRFRKNQQGVTAIEYGLIAVAVAILI IAVFYNNQGFLMK MLSVLMTQAYISATESLRTS IQRFRKNQQGVTAIEYGLIAVAVAILI IAVFYNNOGFLMK MLSVLMTQAYISATESLRTSIQRFRKNQQGVTAIEYGLIAVAVAILI IAVFYNNQGFLMK MLSVLMTQAY ISATESLRTSIQRFRKNQQGVTAIEYGLIAVAVAILI IAVFYNNOGFLMK MLSVLMTQAYISATESLRTS IQRFRKNQQGVTAIEYGLIAVAVAILI IAVFYNNQGFLMK MLSVLMTQAYISATESLRTSIQRFRKNQQGVTAIEYGLIAVAVAILI IAVFYNNQGFLMK MLSVLMTQAYISATESLRTSIQRFRKNQQGVTAIEYGLIAVAVAILI IAVFYNNQGFLMK MLSVLMTQAYISATESLRTS IQRFRKNQQGVTAIEYGLIAVAVAILI IAVFYNNOGFLMK MLSVLMTQAY ISATESLRTS IQRFRKNQQGVTAIEYGLIAVAVAILI IAVFYNNQGFLMK MLSVLMTQAYISATESLRTSIORFRKNQOGVTAIEYGLIAVAVAILI IAVFYNNOGFLMK MLSVLMTQAYISATESLRTS IQRFRKNQQGVTAIEYGLIAVAVAILI IAVFYNNQGFLMK --------VYISTTEILCSS IQRFKKNQRGVTAIEYGLIAVAVAVLI IAAFYSENSF IKK --------VYISTTEILCSSIQRFKKNQRGVTAIEYGLIAVAVAVLI IAAFYSENSF IKK --------VYISTTEILCSS IQRFKKNQRGVTAIEYGLIAVAVAVLI IAAFYSENSF IKK --------VYISTTEILCSS IQRFKKNQRGVTAIEYGLIAVAVAVLI IAAFYSENSF IKK $. * * *: * * *:: * * * * *: * * *: * * * * * * * * * * * * * * *: * * * * . * * .:: . *: *$

LKTKF SDLATGISAQSVSFNN*---LKTKF SDLATGISAQSVSFNN*---LKTKFSDLATGISAQSVSFNN*---LKTKF SDLATGISAQSVSFNN*---LKTKF SDLATGISAQSVSFNN*---LKTKFSDLATGISAQSVSFNN*---LKTKFSDLATGISAQSVSFNN*---LKTKF SDLATGISAQSVSFNN*---LKTKF SDLATGISAQSVSFNN*---LKTKFSDLATGISAQSVSFNN*---LKTKF SDLATGISAQSVSFNN*---LQSKF SNLASGINGTANELNNFHKNS LQSKFSNLASGINGTANELNNFHKNS LQSKF SNLASGINGTANELNNFHKNS LQSKF SNLASGINGTANELNNFHKNS $*:: * * *: * *: * * \ldots:$. .:**

\section{9) Flp3 (HD1310)}

$35000 \mathrm{HP}$

NZS1

NZS2

NZS3

NZS 4

NZV1

82-029362

6644

HD 183

HMC 46

HMC5 6

33921

CIP5 42

DMC 64

DMC 111

$35000 \mathrm{HP}$
MLITIITKPYLSMKETLISWFNCFKINQKGVTAIEYGLIAVAVAILI IAVFYSESGFLFA MLITIITKPYLSMKETLISWFNCFKINQKGVTAIEYGLIAVAVAILI IAVFYSESGFLFA MLITIITKPYLSMKETLISWFNCFKINOKGVTAIEYGLIAVAVAILIIAVFYSESGFLFA MLITIITKPYLSMKETLISWFNCFKINOKGVTAIEYGLIAVAVAILIIAVFYSESGFLFA MLITI ITKPYLSMKETLISWFNCFKINQKGVTAIEYGLIAVAVAILI IAVFYSESGFLFA MLITIITKPYLSMKETLISWFNCFKINQKGVTAIEYGLIAVAVAILI IAVFYSESGFLFA MLITIITKPYLSMKETLISWFNCFKINQKGVTAIEYGLIAVAVAILI IAVFYSESGFLFA MLITIITKPYLSMKETLISWFNCFKINQKGVTAIEYGLIAVAVAILIIAVFYSESGFLFA MLITIITKPYLSMKETLISWFNCFKINQKGVTAIEYGLIAVAVAILIIAVFYSESGFLFA MLITI ITKPYLSMKETLISWFNCFKINQKGVTAIEYGLIAVAVAILI IAVFYSESGFLFA MLITI ITKPYLSMKETLISWFNCFK INQKGVTAIEYGLIAVAVAILI IAVFYSESGFLFA MLSTLTTKAYISTSETLRNKMKTFRQDQQGVTAIEYGLIAVAVAVLI IAVFYSEDGFLFK MLSTLTTKAYISTSETLRNKMKTFRQDQQGVTAIEYGLIAVAVAVLI IAVFYSEDGFLFK MLSTLTTKAYISTSETLRNKMKTFRQDQQGVTAIEYGLIAVAVAVLI IAVFYSEDGFLFK MLSTLTTKAYISTSETLRNKMKTFRQDQQGVTAIEYGLIAVAVAVLI IAVFYSEDGFLFK $* * *: * * *: * . * * * .:::^{*}:: *: * * * * * * * * * * * * * *: * * * * * * * * * . * * * *$

LKEKFFQLEGGVGKAAPDSYLLNFNKGRL* 


$\begin{array}{ll}\text { NZS1 } & \text { LKEKFFQLEGGVGKAAPDSYLLNFNKGRL* } \\ \text { NZS2 } & \text { LKEKFFQLEGGVGKAAPDSYLLNFNKGRL* } \\ \text { NZS3 } & \text { LKEKFFQLEGGVGKAAPDSYLLNFNKGRL* } \\ \text { NZS4 } & \text { LKEKFFQLEGGVGKAAPDSYLLNFNKGRL* } \\ \text { NZV1 } & \text { LKEKFFQLEGGVGKAAPDSYLLNFNKGRL* } \\ 82-029362 & \text { LKEKFFQLEGGVGKAAPDSYLLNFNKGRL* } \\ 6644 & \text { LKEKFFQLEGGVGKAAPDSYLLNFNKGRL* } \\ \text { HD183 } & \text { LKEKFFQLEGGVGKAAPDSYLLNFNKGRL* } \\ \text { HMC46 } & \text { LKEKFFQLEGGVGKAAPDSYLLNFNKGRL* } \\ \text { HMC56 } & \text { LKEKFFQLEGGVGKAAPDSYLLNFNKGRL* } \\ 33921 & \text { LREKFFHLEVGLGNAAPDGDLI-------- } \\ \text { CIP542 } & \text { LREKFFHLEVGLGNAAPDGDLI-------- } \\ \text { DMC64 } & \text { LREKFFHLEVGLGNAAPDGDLI-------- } \\ \text { DMC111 } & \text { LREKFFHLEVGLGNAAPDGDLI-------- } \\ & \text { * **** ** * * * * * * * : }\end{array}$

\section{0) Hfq (HD0741)}

$35000 \mathrm{HP} \quad$ MAKGQSLQDPYLNALRRERIPVSIYLVNGIKLQGQIESFDQFIILLKNTVSQMVYKHAIS

33921 MAKGQSLQDPYLNALRRERIPVS IYLVNGIKLQGQIESFDQFVILLKNTVSQMVYKHAIS CIP542 MAKGQSLQDPYLNALRRERIPVSIYLVNGIKLQGQIESFDQFVILLKNTVSQMVYKHAIS DMC64 MAKGQSLQDPYLNALRRERIPVSIYLVNGIKLQGQIESFDQFVILLKNTVSQMVYKHAIS DMC111 MAKGQSLQDPYLNALRRERIPVSIYLVNGIKLQGQIESFDQFVILLKNTVSQMVYKHAIS

$35000 \mathrm{HP}$ TVVPARSISHNNNGSSOAOAPOOAVOTTOPVEAIVATDKME* 33921 TVVPARSISHNNNGSSQAQAPQQAVQTTQPVEATVATDKME* CIP542 TVVPARSISHNNNGSSQAQAPQQAVQTTQPVEATVATDKME* DMC64 TVVPARSISHNNNGSSQAQAPQQAVQTTQPVEATVATDKME* DMC111 TVVPARSISHNNNGSSQAQAPQQAVQTTQPVEATVATDKME* $* * * * * * * * * * * * * * * * * * * * * * * * * * * * * * * * * * * * * * * * *$

Note: The Hfq sequence of NZS1, NZS2, NZS3, NZS4, NZV1, 82-029362, 6644, HD183, HMC46, and HMC56 were identical to that of $35000 \mathrm{HP}$.

\section{1) HgbA (HD2025)}

$35000 \mathrm{HP}$

$82-029362$

6644

HMC 46

HMC 56

33921

CIP5 42

DMC 64

DMC 111

$35000 \mathrm{HP}$

82-029362

6644

HMC 46

HMC 56

33921

CIP5 42

DMC 64

DMC 111

$35000 \mathrm{HP}$

82-029362

6644

HMC 46

HMC 56

33921

CIP 542

DMC 64

DMC 111

$35000 \mathrm{HP}$
MKANKLSAITLCILGYAHTVYAESNMQTEKLETIVVSSEDDSVHNKNVGEIKKNAKALSK MKANKLSAITLCILGYAHTVYAESNMOTEKLETIVVSSEDDSVHNKNVGEIKKNAKALSK MKANKLSAITLCILGYAHTVYAESNMQTEKLETIVVSSEDDSVHNKNVGEIKKNAKALSK MKANKLSAITLCILGYAHTVYAESNMOTEKLETIVVSSEDDSVHNKNVGEIKKNAKALSK MKANKLSAITLCILGYAHTVYAESNMQTEKLETIVVSSEDDSVHNKNVGEIKKNAKALSK MKTNKLSAITLCILGYAHTVYAESNMQTEKLETIVVSSEDDSVHNKNVGEIKKNAKALSK MKTNKLSAITLCILGYAHTVYAESNMOTEKLETIVVSSEDDSVHNKNVGEIKKNAKALSK MKTNKLSAITLCILGYAHTVYAESNMQTEKLETIVVSSEDDSVHNKNVGEIKKNAKALSK MKANKLSAITLCILGYAHTVYAESNMQTEKLETIVVSSEDDSVHNKNVGE IKKNAKALSK

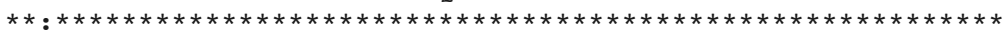

QQVQDSRDLVRYETGVTVVEKGRFGSSGYAIRGVDENRVAVVVDGLHQAETISSQGFKEL QQVQDSRDLVRYETGVTVVEKGRFGSSGYAIRGVDENRVAVVVDGLHQAETISSQGFKEL QQVQDSRDLVRYETGVTVVEKGRFGSSGYAIRGVDENRVAVVVDGLHQAETISSQGFKEL QQVQDSRDLVRYETGVTVVEKGRFGSSGYAIRGVDENRVAVVVDGLHQAETISSQGFKEL OQVQDSRDLVRYETGVTVVEKGRFGSSGYAIRGVDENRVAVVVDGLHOAETISSOGFKEL QQVQDSRDLVRYETGITVVEKGRFGSSGYAIRGVDENRVAVVVDGLHQAETISSQGFKEL QQVQDSRDLVRYETGITVVEKGRFGSSGYAIRGVDENRVAVVVDGLHQAETISSOGFKEL QQVQDSRDLVRYETGITVVEKGRFGSSGYAIRGVDENRVAVVVDGLHQAETISSQGFKEL QQVQDSRDLVRYETGVTVVEKGRFGSSGYAIRGVDENRVAVVVDGLHQAETISSQGFKEL $* * * * * * * * * * * * * * *: * * * * * * * * * * * * * * * * * * * * * * * * * * * * * * * * * * * * * * * * * * * *$

FEGYGNFNNTRNGVEVENLKOAVIQKGADAIRTGSGSLGGTVSFESKDARDYLIDKNYHF FEGYGNFNNTRNGVEVENLKQAVIQKGADAIRTGSGSLGGTVSFESKDARDYLIDKNYHF FEGYGNFNNTRNGVEVENLKQAVIQKGADAIRTGSGSLGGTVSFESKDARDYLIDKNYHF FEGYGNFNNTRNGVEVENLKQAVIQKGADAIRTGSGSLGGTVSFESKDARDYLIDKNYHF FEGYGNFNNTRNGVEVENLKQAVIQKGADAIRTGSGSLGGTVSFESKDARDYLIDKNYHF FEGYGNFNNTRNGVEVENLKOVVIOKGADAIRTGSGSLGGTVSFESKDVRDYLIDKNYHF FEGYGNFNNTRNGVEVENLKQVVIQKGADAIRTGSGSLGGTVSFESKDVRDYLIDKNYHF FEGYGNFNNTRNGVEVENLKQVVIQKGADAIRTGSGSLGGTVSFESKDVRDYLIDKNYHF FEGYGNFNNTRNGVEVENLKQAVIQKGADAIRTGSGSLGGTVSFESKDARDYLIDKNYHF $* * * * * * * * * * * * * * * * * * * * * . * * * * * * * * * * * * * * * * * * * * * * * * * * . * * * * * * * * * * *$ GYKTGYSSADNQKLHSVTAAGRYSDFDLLAVHTQRHGNELRNYGYRHYDGSVVRKEREKA 
$82-029362$

6644

HMC 46

HMC 56

33921

CIP 542

DMC 64

DMC 111

$35000 \mathrm{HP}$

82-029362

6644

HMC 46

HMC 56

33921

CIP5 42

DMC6 4

DMC 111

$35000 \mathrm{HP}$

82-029362

6644

HMC 46

HMC 56

33921

CIP 542

DMC6 4

DMC 111

$35000 \mathrm{HP}$

82-029362

6644

HMC 46

HMC 56

33921

CIP 542

DMC 64

DMC 111

$35000 \mathrm{HP}$

82-029362

6644

HMC 46

HMC 56

33921

CIP 542

DMC 64

DMC 111

$35000 \mathrm{HP}$

82-029362

6644

HMC 46

HMC 56

33921

CIP 542

DMC 64

DMC 111

$35000 \mathrm{HP}$

82-029362

6644

HMC 46

HMC 56

33921

CIP5 42

DMC6 4
GYKTGYSSADNOKLHSVTAAGRYSDFDLLAVHTQRHGNELRNYGYRHYDGSVVRKEREKA GYKTGYSSADNOKLHSVTAAGRYSDFDLLAVHTORHGNELRNYGYRHYDGSVVRKEREKA GYKTGYSSADNQKLHSVTAAGRYSDFDLLAVHTQRHGNELRNYGYRHYDGSVVRKEREKA GYKTGYSSADNQKLHSVTAAGRYSDFDLLAVHTQRHGNELRNYGYRHYDGSVVRKEREKA GYKTGYSSADNQKLHSVTAAGRYSDFDLLAVHTQRHGNELRNYGHRHYDGSVVRKEREKA GYKTGYSSADNQKLHSVTAAGRYSDFDLLAVHTORHGNELRNYGHRHYDGSVVRKEREKA GYKTGYSSADNQKLHSVTAAGRYSDFDLLAVHTQRHGNELRNYGHRHYDGSVVRKEREKA GYKTGYSSADNQKLHSVTAAGRYSDFDLLAVHTQRHGNELRNYGYRHYDGSVVRKEREKA $* * * * * * * * * * * * * * * * * * * * * * * * * * * * * * * * * * * * * * * * * * * * ; * * * * * * * * * * * * * * *$

DPYKITKOSSLIKIGYOLNDTNRFTLGYDDSRNTSRGTDWSNAFTSYNGGPFLKDVRHTN DPYKITKQSSLIKIGYQLNDTNRFTLGYDDSRNTSRGTDWSNAFTSYNGGPFLKDVRHTN DPYKITKQSSLIKIGYQLNDTNRFTLGYDDSRNTSRGTDWSNAFTSYNGGPFLKDVRHTN DPYKITKOSSLIKIGYOLNDTNRFTLGYDDSRNTSRGTDWSNAFTSYNGGPFLKDVRHTN DPYKITKQSSLIKIGYQLNDTNRFTLGYDDSRNTSRGTDWSNAFTSYNGGPFLKDVRHTN DPYKITKOSSLIKIGYOLNDTNRFTLGYDDSRNTSRGTDWSNAFTSYNGGPFLKDVRHTH DPYKITKQSSLIKIGYQLNDTNRFTLGYDDSRNTSRGTDWSNAFTSYNGGPFLKDVRHTH DPYKITKQSSLIKIGYQLNDTNRFTLGYDDSRNTSRGTDWSNAFTSYNGGPFLKDVRHTH DPYKITKQSSLIKIGYQLNDTNRFTLGYDDSRNTSRGTDWSNAFTSYNGGPFLKDVRHTN $\star * * * * * * * * * * * * * * * * * * * * * * * * * * * * * * * * * * * * * * * * * * * * * * * * * * * * * * * * * *$.

DQSNRKNISFVYENFDTNDFWDTLKITHNHQKIKLKARLDEYCDVNGEIDCPAIANPSGL DQSNRKNISFVYENFDTNDFWDTLKITHNHOKIKLKARLDEYCDVNGEIDCPAIANPSGL DQSNRKNISFVYENF DTNDFWDTLKITHNHQKIKLKARLDEYCDVNGEIDCPAIANPSGL DQSNRKN ISFVYENFDTNDFWDTLKITHNHOKIKLKARLDEYCDVNGEIDCPAIANPSGL DQSNRKNISFVYENFDTNDFWDTLKITHNHOKIKLKARLDEYCDVNGEIDCPAIANPSGL DKSNRKN ISVVYENFDTNDFWDTLKITHNHQK I KLKARLDEYCDVNGEIDCPT IANPSGL DKSNRKN ISVVYENFDTNDFWDTLKITHNHOK IKLKARLDEYCDVNGEIDCPTIANPSGL DKSNRKNISVVYENFDTNDFWDTLKITHNHQKI KLKARLDEYCDVNGEIDCPTIANP SGL DQSNRKNISFVYENFDTNDFWDTLKITHNHQKIKLKARLDEYCDVNGEIDCPAIANPSGL $*: * * * * * * * . * * * * * * * * * * * * * * * * * * * * * * * * * * * * * * * * * * * * * * * * * * * * * *:: * * * * * * *$

YINDKGIFLDKHDGEITHKKEGEFNNYFDSKGKEVRVKGFNVDS ILINCDOYDCSKPMOL YINDKGIFLDKHDGEITHKKEGEFNNYFDSKGKEVRVTGFNVDS ILINCDQYDCSKPMOL Y INDKGIFLDKHDGE ITHKKEGEFNNYFDSKGKEVRVTGFNVDS IL INCDQYDCSKPMOL YINDKGIFLDKHDGEITHKKEGEFNNYFDSKGKEVRVTGFNVDS IL INCDQYDCSKPMQI YINDKGIFLDKHDGEITHKKEGEFNNYFDSKGKEVRVTGFNVDS ILINCDQYDCSKPMOL YINDKGIFLDKHDGKITHKKEGEFNNYFDSKGKEVRVKEFNVDS ILINCDOYDCSKPMOL YINDKGIFLDKHDGKITHKKEGEFNNYFDSKGKEVRVKEFNVDS ILINCDQYDCSKPMOL Y INDKGIFLDKHDGKITHKKEGEFNNYFDSKGKEVRVKEFNVDS ILINCDOYDCSKPMOL YINDKGIFLDKHDGEITHKKEGEFNNYFDSKGKEVRVKGFNVDS ILINCDQYDCSKPMQL $* * * * * * * * * * * * * *: * * * * * * * * * * * * * * * * * * * * * * . . * * * * * * * * * * * * * * * * * * * * *$

LSSTNNGYGGSPNKYIYKTYELFEKTMNNGNGKYAVLEIRSSGHEKF SRVYLPSEKGYVE LSSTNNGYGGSPNKY IYKTYELFEKTMNNGNGKYAVLEIRSSGHEKF SRVYLPSEKGYVE LSSTNNGYGGSPNKYIYKTYELFEKTMNNGNGKYAVLEIRSSGHEKF SRVYLPSEKGYVE LSSTNNGYGGSPNKYIYKTYELFEKTMNNGNGKYAVLEIRSSGHEKF SRVYLPSEKGYVE LSSTNNGYGGSPNKYIYKTYELFEKTMNNGNGKYAVLEIRSSGHEKF SRVYLPSEKGYVE LSSTNNGYGNSPNEYKYITYELSEKTMNNGNGKYAVLESRGE-NKKF SRVYLPSEKGYVE LSSTNNGYGNSPNEYKYITYELSEKTMNNGNGKYAVLESRGE-NKKFSRVYLPSEKGYVE LSSTNNGYGNSPNEYKYITYELSEKPMNNGNGKYAVLESRGE-NKKF SRVYLPSEKGYVE LSSTNNGYGGSPNKYIYKTYELFEKTMNNGNGKYAVLEIRSSGHEKF SRVYLPSEKGYVE $* * * * * * * * * * *: * * * * * * * * * * * * * * * * * * * * * \ldots . .: * * * * * * * * * * * * * * *$

NQWKDRDLNTDTQQYNIDLTKSFKLKSVEHNATYGGLYSEVKKSMTNRAGYEAYNRQWWA NQWKDRDLNTDTQQYNIDLTKSFKLKSVEHNATYGGLYSEVKKSMTNRAGYEAYNRQWWA NOWKDRDLNTDTOOYNIDLTKSFKLKSVEHNATYGGLYSEVKKSMTNRAGYEAYNROWWA NQWKDRDLNTDTQQYNIDLTKSFKLKSVEHNATYGGLYSEVKKSMTNRAGYEAYNRQWWA NQWKDRDLNTDTQQYNIDLTKSFKLKSVEHNATYGGLYSEVKKSMTNRAGYEAYNRQWWA NQWRDRDLNTDTQOYNIDLTKSFKLKSVEHNATYGGLYSEVKKSMINRAGYEAYNROWWA NQWRDRDLNTDTQQYNIDLTKSFKLKSVEHNATYGGLYSEVKKSMINRAGYEAYNRQWWA NQWRDRDLNTDTQQYNIDLTKSFKLKSVEHNATYGGLYSEVKKSMINRAGYEAYNRQWWA NQWKDRDLNTDTQQYNIDLTKSFKLKSVEHNATYGGLYSEVKKSMTNRAGYEAYNRQWWA $* * *: * * * * * * * * * * * * * * * * * * * * * * * * * * * * * * * * * * * * * * * * * * * * * * * * * * * * * * *$

NIFFGKENNKPNKCQPYNGNSFTTLCSHEDRLFSFLIPVKTKTGALYVTDKIKLNDKVNL NIFFGKENNKPNKCQPYNGNSFTTLCSHEDRLFSFLIPVKTKTGALYVTDKIKLNDKVNL NIFFGKENNKPNKCQPYNGNSFTTLCSHEDRLFSFLIPVKTKTGALYVTDKIKLNDKVNL NIFFGKENNKPNKCOPYNGNSFTTLCSHEDRLFSFLIPVKTKTGALYVTDKIKLNDKVNL NIFFGKENNKPNKCQPYNGNSFTTLCSHEDRLFSFLIPVKTKTGALYVTDKIKLNDKVNL NIFFGEENGKPNKCQPYNGNSFTTLCSHEDRLFSFLIPVKTKTGALYVTDKIKLNDKVNL NIFFGEENGKPNKCOPYNGNSFTTLCSHEDRLFSFLIPVKTKTGALYVTDKIKLNDKVNL NIFFGEENGKPNKCQPYNGNSFTTLCSHEDRLFSFLIPVKTKTGALYVTDKIKLNDKVNL 
$35000 \mathrm{HP}$

82-029362

6644

HMC 46

HMC 56

33921

CIP 542

DMC6 4

DMC 111

$35000 \mathrm{HP}$

82-029362

6644

HMC 46

HMC 56

33921

CIP 542

DMC 64

DMC 111

$35000 \mathrm{HP}$

82-029362

6644

HMC 46

HMC 56

33921

CIP 542

DMC 64

DMC 111

$35000 \mathrm{HP}$

82-029362

6644

HMC 46

HMC 56

33921

CIP 542

DMC 64

DMC 111

$35000 \mathrm{HP}$

82-029362

6644

HMC 46

HMC 56

33921

CIP5 42

DMC 64

DMC 111

$35000 \mathrm{HP}$

82-029362

6644

HMC 46

HMC56

33921

CIP5 42

DMC 64

DMC 111

$35000 \mathrm{HP}$

$82-029362$

6644

HMC 46
DVAYRYDRIKHDPKYIPGTTPKLPTDLILGRFIEFKPKNTYATODEKNENAEKNAVYLAS DVAYRYDRIKHDPKY IPGTTPKLPTDLILGRF IEFKPKNTYATQDEKNENAEKNAVYLAS DVAYRYDRIKHDPKY IPGTTPKLPTDLILGRF IEFKPKNTYATQDEKNENAEKNAVYLAS DVAYRYDRIKHDPKYIPGTTPKLPTDLILGRF IEFKPKNTYATODEKNENAEKNAVYLAS DVAYRYDRIKHDPKY IPGTTPKLPTDLILGRF IEFKPKNTYATQDEKNENAEKNAVYLAS DLAYRYDRIKHDPKYIPGTTPKLPTDLILGRF IELKPKNTYATODEKNENAEKNAVYLAS DLAYRYDRIKHDPKY IPGTTPKLPTDLILGRF IELKPKNTYATQDEKNENAEKNAVYLAS DLAYRYDRIKHDPKYIPGTTPKLPTDLILGRF IELKPKNTYATODEKNENAEKNAVYLAS DVAYRYDRI KHDPKYIPGTTPKLPTDLILGRF IEFKPKNTYATQDEKNENAEKNAVYLAS $*: * * * * * * * * * * * * * * * * * * * * * * * * * * * * * * * *: * * * * * * * * * * * * * * * * * * * * * * * * *$

KKTKF SANSYSATFSFDPMDFLKIQAKYATGFRAPTSDEIYFVFQHPSF S IYPNLYLKAE KKTKF SANSYSATF SFDPMDFLKIQAKYATGFRAPTSDEIYFVFQHPSFS IYPNLYLKAE KKTKFSANSYSATF SFDPMDFLKIQAKYATGFRAPTSDEIYFVFQHPSF SIYPNLYLKAE KKTKF SANSYSATF SFDPMDFLKIQAKYATGFRAPTSDEIYFVFQHPSFSIYPNLYLKAE KKTKF SANSYSATFSFDPMDFLKIOAKYATGFRAPTSDEIYFVFOHPSFS IYPNLYLKAE KKTKF SANSYSATFSFDPMDFLKIQAKYATGFRAPTSDEI YFVFQHPSF S I YPNLDLKAE KKTKF SANSYSATFSFDPMDFLKIQAKYATGFRAPTSDEIYFVFOHPSF S IYPNLDLKAE KKTKF SANSYSATF SFDPMDFLKIQAKYATGFRAPTSDEIYFVFQHPSFS IYPNLDLKAE KKTKF SANSYSATF SFDPMDFLKIQAKYATGFRAPTSDEIYFVFQHPSF S IYPNLYLKAE $* * * * * * * * * * * * * * * * * * * * * * * * * * * * * * * * * * * * * * * * * * * * * * * * * * * * * * * * * * * *$

RSKNKEVAITLHKOKSFLTVNLFOTDYKDFLDLAYLKKGSLPYGNGGSQLETLLYQNVNR RSKNKEVAITLHKQKSFLTVNLFQTDYKDFLDLAYLKKGSLPYGNGGSQLETLLYQNVNR RSKNKEVAITLHKOKSFLTVNLFOTDYKDFLDLAYLKKGSLPYGNGGSOLETLLYONVNR RSKNKEVAITLHKOKSFLTVNLFQTDYKDFLDLAYLKKGSLPYGNGGSQLETLLYQNVNR RSKNKEVAITLHKQKSFLTVNLFQTDYKDFLDLAYLKKGSLPYGNGGSQLETLLYQNVNR RSKNKEVAITLHKQQSFLTVNLFQTDYKDFLDLAYLKKGSLPYGNGGSQLETLLYQNVNR RSKNKEVAITLHKQQSFLTVNLFQTDYKDFLDLAYLKKGSLPYGNGGSQLETLLYQNVNR RSKNKEVAITLHKOQSFLTVNLFOTDYKDFLDLAYLKKGSLPYGNGGSOLETLLYONVNR RSKNKEVAITLHKOKSFLTVNLFQTDYKDFLDLAYLKKGSLPYGNGGSQLETLLYQNVNR $* * * * * * * * * * * * * *: * * * * * * * * * * * * * * * * * * * * * * * * * * * * * * * * * * * * * * * * * * * * * *$

DKARVKGLEVNSKLHLGDVWRTLDGFNLSYKLSLQKGRMSSKVGEEGKORDTNKLDTPMN DKARVKGLEVNSKLHLGDVWRTLDGFNLSYKLSLQKGRMSSKVGEEGKQRDTNKLDTPMN DKARVKGLEVNSKLHLGDVWRTLDGFNLSYKLSLQKGRMSSKVGEEGKQRDTNKLDTPMN DKARVKGLEVNSKLHLGDVWRTLDGFNLSYKLSLOKGRMSSKVGEEGKORDTNKLDTPMN DKARVKGLEVNSKLHLGDVWRTLDGFNLSYKLSLQKGRMSSKVGEEGKQRDTNKLDTPMN DKARVKGLEVNSRLHLGDVWGVLDGFNLSYKLSLQKGRMS SKVGEEGKQRDTNKLDTPMN DKARVKGLEVNSRLHLGDVWGVLDGFNLSYKLSLOKGRMSSKVGEEGKORDTNKLDTPMN DKARVKGLEVNSRLHLGDVWGVLDGFNLSYKLSLQKGRMSSKVGEEGKQRDTNKLDTPMN DKARVKGLEVNSKLHLGDVWRTLDGFNLSYKLSLQKGRMSSKVGEEGKORDTNKLDTPMN $* * * * * * * * * * * *: * * * * * * * . . * * * * * * * * * * * * * * * * * * * * * * * * * * * * * * * * * * * * * *$

AIQPQTHVVGVGYEHPQEKFGVDMYLTHASAKKEKDTFNMFYDGKDQKDQHIKWRSDRYT AIQPQTHVVGVGYEHPQEKFGVDMYLTHASAKKEKDTFNMFYDGKDQKDQHIKWRSDRYT AIQPQTHVVGVGYEHPQEKFGVDMYLTHASAKKEKDTFNMFYDGKDQKDQH I KWRSDRYT AIQPQTHVVGVGYEHPQEKFGVDMYLTHASAKKEKDTFNMFYDGKDQKDQHIKWRSDRYT AIQPOTHVVGVGYEHPQEKFGVDMYLTHASAKKEKDTFNMFYDGKDQKDQH IKWRSDRYT VIQPQTHVVGLGYEHTQEKFGVDMYLTHASAKKEKDTFNMFYDGKDQKDQHIKWRSDSYT VIQPQTHVVGLGYEHTQEKFGVDMYLTHASAKKEKDTF NMFYDGKDQKDOH IKWRSDSYT VIQPQTHVVGLGYEHTQEKFGVDMYLTHASAKKEKDTFNMFYDGKDQKDQHIKWRSDSYT AIQPQTHVVGVGYEHPQEKFGVDMYLTHASAKKEKDTFNMFYDGKDQKDQHIKWRSDRYT $* * * * * * * * * \cdot * * * * \quad * * * * * * * * * * * * * * * * * * * * * * * * * * * * * * * * * * * * * * * * * * * *$

LVDLIAYVKPVKNVTLRAGVYNLTNREYGTWDS IRS IRPFGTTNLINQETGKGIKRFNAP LVDLIAYVKPVKNVTLRAGVYNLTNREYGTWDS IRS IRPFGTTNLINOETGKGIKRFNAP LVDLIAYVKPVKNVTLRAGVYNLTNREYGTWDSIRS IRPFGTTNLINQETGKGIKRFNAP LVDLIAYVKPVKNVTLRAGVYNLTNREYGTWDS IRS IRPFGTTNLINQETGKGIKRFNAP LVDLIAYVKPVKNVTLRAGVYNLTNREYGTWDS IRS IRPFGTTNLINQETGKGIKRFNAP LVDLIAY IKPVKNVTLRAGVYNLTNREYGTWDS IRS IRPFGTTNLINQKTGKGI KRFNAP LVDLIAY IKPVKNVTLRAGVYNLTNREYGTWDSIRSIRPFGTTNLINOKTGKGIKRFNAP LVDLIAY IKPVKNVTLRAGVYNLTNREYGTWDS IRS IRPFGTTNLINQKTGKGIKRFNAP LVDLIAYVKPVKNVTLRAGVYNLTNREYGTWDS IRS IRPFGTTNLINQETGKGIKRFNAP $* * * * * * *: * * * * * * * * * * * * * * * * * * * * * * * * * * * * * * * * * * * * * * * *: * * * * * * * * * * *$

GRNFRVNAEITF* GRNFRVNAEITF* GRNFRVNAEITF* GRNFRVNAEITF * 


\begin{abstract}
HMC56 GRNFRVNAEITF *
33921 GRNFRVNAEITF *

CIP542 GRNFRVNAEITF*

DMC6 4 GRNFRVNAEITF*

DMC111 GRNFRVNAEITF *

$* * * * * * * * * * * * *$

Note: The HgbA sequence of NZS1, NZS2, NZS3, NZS4, and NZV1 were identical to that of $35000 \mathrm{HP}$.
\end{abstract}

\title{
12) LspA1 (HD1505)
}

$35000 \mathrm{HP}$

NZS1

NZS2

NZS 3

NZS 4

NZV1

$82-029362$

6644

HD 183

HMC 46

HMC 56

$35000 \mathrm{HP}$

NZS 1

NZS2

NZS3

NZS 4

NZV1

$82-029362$

6644

HD 183

HMC 46

HMC 56

$35000 \mathrm{HP}$

NZS 1

NZS2

NZS3

NZS 4

NZV1

82-029362

6644

HD 183

HMC 46

HMC 56

$35000 \mathrm{HP}$

NZS 1

NZS2

NZS 3

NZS 4

NZV1

82-029362

6644

HD 183

HMC 46

HMC 56

$35000 \mathrm{HP}$

NZS 1

NZS2

NZS3

NZS4

NZV1

82-029362

6644
MNNKRYKLIFSKVKNCLVPVAENIKSASGNSGSSSNSKIAEDQEEEPDSLACSLSPLSSS MNNKRYKLIFSKVKNCLVPVAENIKSASGNSGSSSNSKIAEDQEEEPDSLACSLSPLSSS MNNKRYKLIFSKVKNCLVPVAENIKSASGNSGSSSNSKIAEDOEEEPDSLACSLSPLSSS MNNKRYKLIFSKVKNCLVPVAENIKSASGNSGSSSNSKIAEDQEEEPDSLACSLSPLSSS MNNKRYKLIFSKVKNCLVPVAENIKSASGNSGSSSNSKIAEDQEEEPDSLACSLSPLSSS MNNKRYKLIFSKVKNCLVPVAENIKSASGNSGSSSNSKIAEDQEEEPDSLACSLSPLSSS MNNKRYKLIFSKVKNCLVPVAENIKSASGNSGSSSNSKIAEDQEEEPDSLACSLSPLSSS MNNKRYKLIFSKVKNCLVPVAENIKSASGNSGSSSNSKIAEDQEEEPDSLACSLSPLSSS MNNKRYKLIFSKVKNCLVPVAENIKSASGNSGSSSNSKIAEDQEEEPDSLACSLSPLSSS MNNKRYKLIFSKVKNCLVPVAENIKSASGNSGSSSNSKIAEDQEEEPDSLACSLSPLSSS MNNKRYKLIFSKVKNCLVPVAENIKSASGNSGSSSNSKIAEDQEEEPDSLACSLSPLSSS

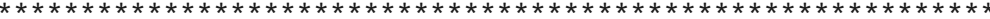

IHLGLHNHSPLKVFKGKSLSVVLLSLMPATPLLAQQNYAEALNGKVYVDSOHSSTRIYEQ IHLGLHNHSPLKVFKGKSLSVVLLSLMPATPLLAQQNYAEALNGKVYVDSQHSSTRIYEQ IHLGLHNHSPLKVFKGKSLSVVLLSLMPATPLLAQQNYAEALNGKVYVDSOHSSTRIYEQ IHLGLHNHSPLKVFKGKSLSVVLLSLMPATPLLAQQNYAEALNGKVYVDSQHSSTRIYEQ IHLGLHNHSPLKVFKGKSLSVVLLSLMPATPLLAQQNYAEALNGKVYVDSOHSSTRIYEQ IHLGLHNHSPLKVFKGKSLSVVLLSLMPATPLLAQQNYAEALNGKVYVDSQHSSTRIYEQ IHLGLHNHSPLKVFKGKSLSVVLLSLMPATPLLAQQNYAEALNGKVYVDSQHSSTRIYEQ IHLGLHNHSPLKVFKGKSLSVVLLSLMPATPLLAQQNYAEALNGKVYVDSQHSSTRIYEQ IHLGLHNHSPLKVFKGKSLSVVLLSLMPATPLLAOONYAEALNGKVYVDSOHSSTRIYEQ IHLGLHNHSPLKVFKGKSLSVVLLSLMPATPLLAQQNYAEALNGKVYVDSOHSSTRIYEQ IHLGLHNHSPLKVFKGKSLSVVLLSLMPATPLLAQONYAEALNGKVYVDSOHSSTRIYEQ $* * * * * * * * * * * * * * * * * * * * * * * * * * * * * * * * * * * * * * * * * * * * * * * * * * * * * * * * * * * * *$

KTNDNSKDGIVVVEIANPEVDGVSDNRFKEFNIPNSAVFNNSRTESTSQLVGKLHANIQL KTNDNSKDGIVVVEIANPEVDGVSDNRF KEFNIPNSAVFNNSRTESTSQLVGKLHANIQL KTNDNSKDGIVVVEIANPEVDGVSDNRFKEFNIPNSAVFNNSRTESTSOLVGKLHANIOL KTNDNSKDGIVVVEIANPEVDGVSDNRFKEFNIPNSAVFNNSRTESTSQLVGKLHANIQL KTNDNSKDGIVVVEIANPEVDGVSDNRFKEFNIPNSAVFNNSRTESTSQLVGKLHANIQL KTNDNSKDGIVVVEIANPEVDGVSDNRFKEFNIPNSAVFNNSRTESTSOLVGKLHANIOL KTNDNSKDGIVVVEIANPEVDGVSDNRFKEFNIPNSAVFNNSRTESTSQLVGKLHANIQL KTNDNSKDGIVVVEIANPEVDGVSDNRFKEFNIPNSAVFNNSRTESTSOLVGKLHANIOI KTNDNSKDGIVVVEIANPEVDGVSDNRFKEFNIPNSAVFNNSRTESTSQLVGKLHANIQI KTNDNSKDGIVVVEIANPEVDGVSDNRFKEFNIPNSAVFNNSRTESTSQLVGKLHANIQL KTNDNSKDGIVVVEIANPEVDGVSDNRFKEFNIPNSAVFNNSRTESTSQLVGKLHANIQL $* * * * * * * * * * * * * * * * * * * * * * * * * * * * * * * * * * * * * * * * * * * * * * * * * * * * * * * * * * * * * *$

QKEAKLILNQVTGDHESNIQGALEVAGKKADLIIVNPNGITLNGVKTINTDRFVVSTSDI OKEAKLILNOVTGDHESNIOGALEVAGKKADLI IVNPNGITLNGVKTINTDRFVVSTSDI QKEAKLILNQVTGDHESNIQGALEVAGKKADLIIVNPNGITLNGVKTINTDRFVVSTSDI QKEAKLILNQVTGDHESNIQGALEVAGKKADLI IVNPNGITLNGVKTINTDRFVVSTSDI QKEAKLILNQVTGDHESNIQGALEVAGKKADLI IVNPNGITLNGVKTINTDRFVVSTSDI QKEAKLILNQVTGDHESNIQGALEVAGKKADLI IVNPNGITLNGVKTINTDRFVVSTSDI QKEAKLILNQVTGDHESNIQGALEVAGKKADLI IVNPNGITLNGVKTINTDRFVVSTSDI QKEAKLILNQVTGDHESNIQGALEVAGKKADLI IVNPNGITLNGVKTINTDRFVVSTSDI OKEAKLILNQVTGDHESNIQGALEVAGKKADLI IVNPNGITLNGVKTINTDRFVVSTSDI QKEAKLILNQVTGDHESNIQGALEVAGKKADLI IVNPNGITLNGVKTINTDRFVVSTSDI QKEAKLILNQVTGDHESNIQGALEVAGKKADLIIVNPNGITLNGVKTINTDRFVVSTSDI $* * * * * * * * * * * * * * * * * * * * * * * * * * * * * * * * * * * * * * * * * * * * * * * * * * * * * * * * * * * *$

IPHRENGLLSVRNGKVTIDKGGVATNGLSHFEVVARNIDQKGKITVAKTENQKSVNPANI IPHRENGLLSVRNGKVTIDKGGVATNGLSHFEVVARNIDQKGKITVAKTENQKSVNPANI IPHRENGLLSVRNGKVTIDKGGVATNGLSHFEVVARNIDQKGKITVAKTENQKSVNPANI IPHRENGLLSVRNGKVTIDKGGVATNGLSHFEVVARNIDOKGKITVAKTENOKSVNPANI IPHRENGLLSVRNGKVTIDKGGVATNGLSHFEVVARNIDQKGKITVAKTENQKSVNPAN I IPHRENGLLSVRNGKVTIDKGGVATNGLSHFEVVARNIDOKGKITVAKTENOKSVNPAN I IPHRENGLLSVRNGKVTIDKGGVATNGLSHFEVVARNIDQKGKITVAKTENQKSVNPANI IPHRENGLLSVRNGKVTIDKGGVATNGLSHFEVVARNIDQKGKITVAKTENQKSVNPANI 
HD183

HMC 46

HMC 56

$35000 \mathrm{HP}$

NZS 1

NZS2

NZS 3

NZS4

NZV1

82-029362

6644

HD 183

HMC 46

HMC 56

$35000 \mathrm{HP}$

NZS 1

NZS2

NZS 3

NZS 4

NZV1

82-029362

6644

HD 183

HMC 46

HMC 56

$35000 \mathrm{HP}$

NZS 1

NZS2

NZS 3

NZS 4

NZV1

82-029362

6644

HD 183

HMC 46

HMC 56

$35000 \mathrm{HP}$

NZS 1

NZS2

NZS 3

NZS 4

NZV1

82-029362

6644

HD 183

HMC 46

HMC 56

$35000 \mathrm{HP}$

NZ 1

NZS2

NZS 3

NZS 4

NZV1

82-029362

6644

HD 183

HMC 46

HMC 56

$35000 \mathrm{HP}$

NZS 1

NZS2
IPHRENGLLSVRNGKVTIDKGGVATNGLSHFEVVARNIDQKGKITVAKTENQKSVNPANI IPHRENGLLSVRNGKVTIDKGGVATNGLSHFEVVARNIDOKGKITVAKTENOKSVNPANI IPHRENGLLSVRNGKVTIDKGGVATNGLSHFEVVARNIDQKGKITVAKTENQKSVNPANI $* * * * * * * * * * * * * * * * * * * * * * * * * * * * * * * * * * * * * * * * * * * * * * * * * * * * * * * * * * * *$

TFAAGSLNYNLKTREATPISSGTSRTSDTPAISADSAGSMYGSNIKFVVTDKGAGVKHKG TFAAGSLNYNLKTREATPISSGTSRTSDTPAISADSAGSMYGSNIKFVVTDKGAGVKHKG TFAAGSLNYNLKTREATPISSGTSRTSDTPAISADSAGSMYGSNIKFVVTDKGAGVKHKG TFAAGSLNYNLKTREATPISSGTSRTSDTPAISADSAGSMYGSNIKFVVTDKGAGVKHKG TFAAGSLNYNLKTREATPISSGTSRTSDTPAISADSAGSMYGSNIKFVVTDKGAGVKHKG TFAAGSLNYNLKTREATPISSGTSRTSDTPAISADSAGSMYGSNIKFVVTDKGAGVKHKG TFAAGSLNYNLKTREATPISSGTSRTSDTPAISADSAGSMYGSNIKFVVTDKGAGVKHKG TFAAGSLNYNLKTREATPISSGTSRTSDTPAISADSAGSMYGSNIKFVVTDKGAGVKHKG TFAAGSLNYNLKTREATPISSGTSRTSDTPAISADSAGSMYGSNIKFVVTDKGAGVKHKG TFAAGSLNYNLKTREATPISSGTSRTSDTPAISADSAGSMYGSNIKFVVTDKGAGVKHKG TFAAGSLNYNLKTREATPISSGTSRTSDTPAISADSAGSMYGSNIKFVVTDKGAGVKHKG $* * * * * * * * * * * * * * * * * * * * * * * * * * * * * * * * * * * * * * * * * * * * * * * * * * * * * * * * * * * *$

I IFSENDINIKMDGGNASLKELYAKKDIDILAKDIELTEKGOLOANNKI ILNSTGKINLR I IF SENDINIKMDGGNASLKELYAKKDIDILAKD IELTEKGQLQANNKI ILNSTGKINLR I IFSENDINIKMDGGNASLKELYAKKDIDILAKDIELTEKGQLQANNKI ILNSTGKINLR I IF SENDINIKMDGGNASLKELYAKKDIDILAKDIELTEKGQLQANNKI ILNSTGKINLR IIFSENDINIKMDGGNASLKELYAKKDIDILAKDIELTEKGQLQANNKI ILNSTGKINLR IIFSENDINIKMDGGNASLKELYAKKDIDILAKDIELTEKGQLQANNKI ILNSTGKINLR I IF SENDINIKMDGGNASLKELYAKKDIDILAKDIELTEKGQLQANNKI ILNSTGKINLR IIFSENDINI KMDGGNASLKELYAKKDIDILAKDIELTEKGQLQANNKI ILNSTGKINLR I IF SENDINI KMDGGNASLKELYAKKDIDILAKD IELTEKGQLQANNKI ILNSTGK INLR I IF SENDINI KMDGGNASLKELYAKKDIDILAKDIELTEKGQLQANNKI ILNSTGKINLR I IF SENDINI KMDGGNASLKELYAKKDIDILAKDIELTEKGQLQANNKI ILNSTGKINLR $* * * * * * * * * * * * * * * * * * * * * * * * * * * * * * * * * * * * * * * * * * * * * * * * * * * * * * * * * * * *$

NASEVSADNVNVKSENLALENASMSANSLDVIVTKIEVNRSSKVSAGTANIKASNITLDG NASEVSADNVNVKSENLALENASMSANSLDVIVTKIEVNRSSKVSAGTANIKASNITLDG NASEVSADNVNVKSENLALENASMSANSLDVIVTKIEVNRSSKVSAGTANIKASNITLDG NASEVSADNVNVKSENLALENASMSANSLDVIVTKIEVNRSSKVSAGTANIKASNITLDG NASEVSADNVNVKSENLALENASMSANSLDVIVTKIEVNRSSKVSAGTANIKASNITLDG NASEVSADNVNVKSENLALENASMSANSLDVIVTKIEVNRSSKVSAGTANIKASNITLDG NASEVSADNVNVKSENLALENASMSANSLDVIVTKIEVNRSSKVSAGTANIKASNITLDG NASEVSADNVNVKSENLALENASMSANSLDVIVTKIEVNRSSKVSAGTANIKASNITLDG NASEVSADNVNVKSENLALENASMSANSLDVIVTKIEVNRSSKVSAGTANIKASNITLDG NASEVSADNVNVKSENLALENASMSANSLDVIVTKIEVNRSSKVSAGTANIKASNITLDG NASEVSADNVNVKSENLALENASMSANSLDVIVTKIEVNRSSKVSAGTANIKASNITLDG $\star * * * * * * * * * * * * * * * * * * * * * * * * * * * * * * * * * * * * * * * * * * * * * * * * * * * * * * * * * * * * * * * * *$

SSVVANKITLNVTNNATLNNOSKLSAKDMELNVTHNITLNNTSKLSAOKANIKTENLTLN SSVVANKITLNVTNNATLNNQSKLSAKDMELNVTHNITLNNTSKLSAQKANIKTENLTLN SSVVANKITLNVTNNATLNNQSKLSAKDMELNVTHNITLNNTSKLSAQKANIKTENLTLN SSVVANKITLNVTNNATLNNQSKLSAKDMELNVTHNITLNNTSKLSAQKANIKTENLTLN SSVVANKITLNVTNNATLNNQSKLSAKDMELNVTHNITLNNTSKLSAQKANIKTENLTLN SSVVANKITLNVTNNATLNNQSKLSAKDMELNVTHNITLNNTSKLSAQKANIKTENLTLN SSVVANKITLNVTNNATLNNOSKLSAKDMELNVTHNITLNNTSKLSAQKANIKTENLTLN SSVVANKITLNVTNNATLNNQSKLSAKDMELNVTHNITLNNTSKLSAQKANIKTENLTLN SSVVANKITLNVTNNATLNNOSKLSAKDMELNVTHNITLNNTSKLSAQKANIKTENLTLN SSVVANKITLNVTNNATLNNQSKLSAKDMELNVTHNITLNNTSKLSAQKANIKTENLTLN SSVVANKITLNVTNNATLNNOSKLSAKDMELNVTHNITLNNTSKLSAOKANIKTENLTLN $* * * * * * * * * * * * * * * * * * * * * * * * * * * * * * * * * * * * * * * * * * * * * * * * * * * * * * * * * * * *$

GEASLVAEKLDINAIDKITNNGTIAGLTANITTKALENRDNALILAHQNLNFTVNGSHYV GEASLVAEKLDINAIDKITNNGTIAGLTANITTKALENRDNALILAHQNLNFTVNGSHYV GEASLVAEKLDINAIDKITNNGTIAGLTANITTKALENRDNALILAHONLNFTVNGSHYV GEASLVAEKLDINAIDKITNNGTIAGLTANITTKALENRDNALILAHQNLNFTVNGSHYV GEASLVAEKLDINAIDKITNNGTIAGLTANITTKALENRDNALILAHONLNFTVNGSHYV GEASLVAEKLDINAIDKITNNGTIAGLTANITTKALENRDNALILAHONLNFTVNGSHYV GEASLVAEKLDINAIDKITNNGTIAGLTANITTKALENRDNAL ILAHQNLNFTVNGSHYV GEASLVAEKLDINAIDKITNNGTIAGLTANITTKALENRDNALILAHQNLNFTVNGSHYV GEASLVAEKLDINAIDKITNNGTIAGLTANITTKALENRDNALILAHQNLNFTVNGSHYV GEASLVAEKLDINAIDKITNNGTIAGLTANITTKALENRDNALILAHQNLNFTVNGSHYV GEASLVAEKLDINAIDKITNNGTIAGLTANITTKALENRDNALILAHQNLNFTVNGSHYV

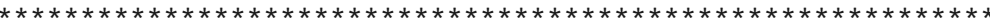

NKGDIVSKDKAIVTFSNNSDFTSNGSKLVDAQNNLTVNVNNFNITQGSEI ILHGNVTLNA NKGDIVSKDKAIVTFSNNSDFTSNGSKLVDAQNNLTVNVNNFNITQGSEI I LHGNVTLNA NKGDIVSKDKAIVTF SNNSDFTSNGSKLVDAQNNLTVNVNNFNITQGSEI ILHGNVTLNA 
NZS 3

NZS 4

NZV1

82-029362

6644

HD 183

HMC 46

HMC 56

$35000 \mathrm{HP}$

NZS 1

NZS2

NZS3

NZS4

NZV1

82-029362

6644

HD183

HMC 46

HMC 56

$35000 \mathrm{HP}$

NZS 1

NZS2

NZS 3

NZS 4

NZV1

82-029362

6644

HD 183

HMC 46

HMC 56

$35000 \mathrm{HP}$

NZS 1

NZS2

NZS 3

NZS 4

NZV1

82-029362

6644

HD 183

HMC 46

HMC 56

$35000 \mathrm{HP}$

NZS 1

NZS2

NZS 3

NZS4

NZV1

82-029362

6644

HD 183

HMC 46

HMC 56

$35000 \mathrm{HP}$

NZS 1

NZS2

NZS 3

NZS 4

NZV1

82-029362

6644

HD183

HMC 46

HMC 56
NKGDIVSKDKAIVTFSNNSDFTSNGSKLVDAONNLTVNVNNFNITOGSEI I LHGNVTLNA NKGDIVSKDKAIVTFSNNSDFTSNGSKLVDAQNNLTVNVNNFNITQGSEI ILHGNVTLNA NKGDIVSKDKAIVTF SNNSDFTSNGSKLVDAQNNLTVNVNNFNITQGSEI I LHGNVTLNA NKGDIVSKDKAIVTFSNNSDFTSNGSKLVDAQNNLTVNVNNFNITQGSE I ILHGNVTLNA NKGDIVSKDKAIVTF SNNSDFTSNGSKLVDAQNNLTVNVNNFNITQGSEI ILHGNVTLNA NKGDIVSKDKAIVTFSNNSDFTSNGSKLVDAQNNLTVNVNNFNITQGSEI ILHGNVTLNA NKGDIVSKDKAIVTF SNNSDFTSNGSKLVDAQNNLTVNVNNFNITQGSEI ILHGNVTLNA NKGDIVSKDKAIVTF SNNSDFTSNGSKLVDAQNNLTVNVNNFNITQGSEI ILHGNVTLNA

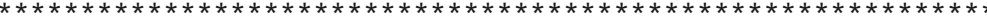

KGNFTNSGNLTTMKELNISNIESF INAGNLTTGKNLEVHSNTTVKNDGKLVSIENLNISS KGNFTNSGNLTTMKELNISNIESF INAGNLTTGKNLEVHSNTTVKNDGKLVS IENLNISS KGNFTNSGNLTTMKELNISNIESF INAGNLTTGKNLEVHSNTTVKNDGKLVS IENLNISS KGNFTNSGNLTTMKELNISNIESF INAGNLTTGKNLEVHSNTTVKNDGKLVSIENLNISS KGNFTNSGNLTTMKELNISNIESF INAGNLTTGKNLEVHSNTTVKNDGKLVS IENLNISS KGNFTNSGNLTTMKELNI SNIESF INAGNLTTGKNLEVHSNTTVKNDGKLVS IENLNISS KGNFTNSGNLTTMKELNISNIESF INAGNLTTGKNLEVHSNTTVKNDGKLVS IENLNISS KGNFTNSGNLTTMKELNISNIESF INAGNLTTGKNLEVHSNTTVKNDGKLVSIENLNISS KGNFTNSGNLTTMKELNISNIESF INAGNLTTGKNLEVHSNTTVKNDGKLVS IENLNISS KGNFTNSGNLTTMKELNISNIESF INAGNLTTGKNLEVHSNTTVKNDGKLVS IENLNISS KGNFTNSGNLTTMKELNISNIESF INAGNLTTGKNLEVHSNTTVKNDGKLVS IENLNISS $* * * * * * * * * * * * * * * * * * * * * * * * * * * * * * * * * * * * * * * * * * * * * * * * * * * * * * * * * * * * *$

KTDFTNNGTLLGLEALKIASGGNFTNASNGSLASNKSLDIYGNNFTNNGTIESVKSLNIT KTDFTNNGTLLGLEALKIASGGNFTNASNGSLASNKSLDIYGNNFTNNGTIESVKSLNIT KTDFTNNGTLLGLEALKIASGGNFTNASNGSLASNKSLDIYGNNFTNNGTIESVKSLNIT KTDFTNNGTLLGLEALKIASGGNFTNASNGSLASNKSLDIYGNNFTNNGTIESVKSLNIT KTDFTNNGTLLGLEALKIASGGNFTNASNGSLASNKSLDIYGNNFTNNGTIESVKSLNIT KTDFTNNGTLLGLEALKIASGGNFTNASNGSLASNKSLDIYGNNFTNNGTIESVKSLNIT KTDFTNNGTLLGLEALKIASGGNFTNASNGSLASNKSLDIYGNNFTNNGTIESVKSLNIT KTDFTNNGTLLGLEALKIASGGNFTNASNGSLASNKSLDIYGNNFTNNGTIESVKSLNIT KTDFTNNGTLLGLEALKIASGGNFTNASNGSLASNKSLDIYGNNFTNNGTIESVKSLNIT KTDFTNNGTLLGLEALKIASGGNFTNASNGSLASNKSLDI YGNNFTNNGTIESVKSLNIT KTDFTNNGTLLGLEALKIASGGNFTNASNGSLASNKSLDIYGNNFTNNGTIESVKSLNIT $* * * * * * * * * * * * * * * * * * * * * * * * * * * * * * * * * * * * * * * * * * * * * * * * * * * * * * * * * * * * * *$

NNYTF INNATIKSYGVLNITSQGNFTNDSNGTVMSHDLLNITSOANI INKNLLAGGQGLN NNYTF INNATI KSYGVLNITSOGNFTNDSNGTVMSHDLLNI TSQAN I INKNLLAGGQGLN NNYTF INNATI KSYGVLNITSQGNFTNDSNGTVMSHDLLNITSQANI INKNLLAGGQGLN NNYTF INNATI KSYGVLNITSOGNFTNDSNGTVMSHDLLNITSOAN I INKNLLAGGOGLN NNYTF INNAT I KSYGVLN I TSOGNFTNDSNGTVMS HDLLN I TSOAN I INKNLLAGGQGLN NNYTF INNAT I KSYGVLN I TSQGNF TNDSNGTVMSHDLLNI TSOAN I INKNLLAGGQGLN NNYTF INNAT I KSYGVLN I TSQGNFTNDSNGTVMS HDLLN ITSQAN I INKNLLAGGQGLN NNYTF INNAT I KSYGVLN I TSOGNFTNDSNGTVMSHDLLNI TSOAN I INKNLLAGGQGLN NNYTF INNATI KSYGVLN I TSQGNFTNDSNGTVMSHDLLNI TSQAN I INKNLLAGGQGLN NNYTF INNAT I KSYGVLN I TSQGNFTNDSNGTVMS HDLLN I TSQAN I INKNLLAGGQGLN NNYTF INNATI KSYGVLNI TSOGNFTNDSNGTVMSHDLLNI TSQAN I INKNLLAGGQGLN $\star * * * * * * * * * * * * * * * * * * * * * * * * * * * * * * * * * * * * * * * * * * * * * * * * * * * * * * * * * * * * * * * * * * * * *$

LTAKGNITNDSNSTAIAVLHSNNDINLNANNKVYNIGE IYSOAGNISVEAKLLHNDVKLS LTAKGNITNDSNSTAIAVLHSNNDINLNANNKVYNIGEIYSQAGNISVEAKLLHNDVKLS LTAKGNITNDSNSTAIAVLHSNNDINLNANNKVYNIGEIYSQAGN ISVEAKLLHNDVKLS LTAKGNITNDSNSTAIAVLHSNNDINLNANNKVYNIGEIYSQAGN ISVEAKLLHNDVKLS LTAKGNITNDSNSTAIAVLHSNNDINLNANNKVYNIGEIYSQAGNISVEAKLLHNDVKLS LTAKGNITNDSNSTAIAVLHSNNDINLNANNKVYNIGEIYSQAGN ISVEAKLLHNDVKLS LTAKGNITNDSNSTAIAVLHSNNDINLNANNKVYNIGEIYSQAGNISVEAKLLHNDVKLS LTAKGNITNDSNSTAIAVLHSNNDINLNANNKVYNIGEIYSOAGNISVEAKLLHNDVKLS LTAKGNITNDSNSTAIAVLHSNNDINLNANNKVYNIGEIYSQAGNISVEAKLLHNDVKLS LTAKGNITNDSNSTAIAVLHSNNDINLNANNKVYNIGEIYSQAGN ISVEAKLLHNDVKLS LTAKGNITNDSNSTAIAVLHSNNDINLNANNKVYNIGEIYSQAGNISVEAKLLHNDVKLS $* * * * * * * * * * * * * * * * * * * * * * * * * * * * * * * * * * * * * * * * * * * * * * * * * * * * * * * * * * * *$

GNITTTTKSGNATVKTNS IGGGLHDANS IRVGELTLNGKFADLDNQLKVALRGKIYAGSN GNITTTTKSGNATVKTNS IGGGLHDANS IRVGELTLNGKF ADLDNQLKVALRGKIYAGSN GN ITTTTKSGNATVKTNS IGGGLHDANS IRVGELTLNGKFADLDNQLKVALRGKIYAGSN GNITTTTKSGNATVKTNS I GGGLHDANS IRVGELTLNGKFADLDNQLKVALRGKIYAGSN GNITTTTKSGNATVKTNS IGGGLHDANS IRVGELTLNGKFADLDNOLKVALRGKIYAGSN GNITTTTKSGNATVKTNS IGGGLHDANS IRVGELTLNGKFADLDNQLKVALRGKIYAGSN GNITTTTKSGNATVKTNS IGGGLHDANS IRVGELTLNGKFADLDNOLKVALRGKIYAGSN GNITTTTKSGNATVKTNS IGGGLHDANS IRVGELTLNGKFADLDNQLKVALRGKIYAGSN GNITTTTKSGNATVKTNS IGGGLHDANS IRVGELTLNGKFADLDNQLKVALRGKIYAGSN GNITTTTKSGNATVKTNS I GGGLHDANS IRVGELTLNGKFADLDNOLKVALRGKIYAGSN GNITTTTKSGNATVKTNSIGGGLHDANS IRVGELTLNGKFADLDNQLKVALRGKIYAGSN 


\section{$35000 \mathrm{HP}$ \\ NZS 1 \\ NZS2 \\ NZS 3 \\ NZS4 \\ NZV1 \\ 82-029362 \\ 6644 \\ HD 183 \\ HMC 46 \\ HMC 56}

$35000 \mathrm{HP}$

NZS 1

NZS2

NZS 3

NZS4

NZV1

82-029362

6644

HD 183

HMC 46

HMC 56

$35000 \mathrm{HP}$

NZS 1

NZS2

NZS 3

NZS 4

NZV1

82-029362

6644

HD 183

HMC 46

HMC 56

$35000 \mathrm{HP}$

NZS 1

NZS2

NZS3

NZS4

NZV1

82-029362

6644

HD 183

HMC 46

HMC 56

$35000 \mathrm{HP}$

NZS1

NZS2

NZS 3

NZS 4

NZV1

82-029362

6644

HD 183

HMC 46

HMC 56

$35000 \mathrm{HP}$

NZS 1

NZS2

NZS 3

NZS 4

NZV1
$* * * * * * * * * * * * * * * * * * * * * * * * * * * * * * * * * * * * * * * * * * * * * * * * * * * * * * * * * * * *$

LTFKAKEGEKEQKSTAQAKI INRGTINVKNKLEYGSNVDVENNMRSMQVNLYEKIFNGDN LTFKAKEGEKEQKSTAQAK I INRGT INVKNKLEYGSNVDVENNMHSMOVNLYEKIFNGDN LTFKAKEGEKEQKSTAQAKI INRGT INVKNKLEYGSNVDVENNMHSMOVNLYEKIFNGDN LTFKAKEGEKEQKSTAQAKI INRGTINVKNKLEYGSNVDVENNMHSMQVNLYEKIFNGDN LTFKAKEGEKEQKSTAQAKI INRGTINVKNKLEYGSNVDVENNMHSMQVNLYEKIFNGDN LTFKAKEGEKEQKSTAQAKI INRGTINVKNKLEYGSNVDVENNMHSMQVNLYEKIFNGDN LTFKAKEGEKEQKSTAQAK I INRGT INVKNKLEYGSNVDVENNMHSMOVNLYEKIFNGDN LTFKAKEGEKEQKSTAQAKI INRGT INVKNKLEYGSNVDVENNMHSMQVNLYEKIFNGDN LTFKAKEGEKEOKSTAOAKI INRGT INVKNKLEYGSNVDVENNMRSMOVNLYEKIFNGDN LTFKAKEGEKEQKSTAQAKI INRGTINVKNKLEYGSNVDVENNMHSMQVNLYEKIFNGDN LTFKAKEGEKEQKSTAQAKI INRGTINVKNKLEYGSNVDVENNMHSMQVNLYEKIFNGDN

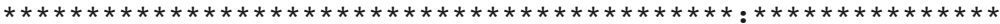

PITLTLKNGVTFAKDFSNRRRRASNDGEGTNKKTFDNVAHLIEEAFSGYSNGNDHRASDD PITLTLKNGVTFAKDFSGRR-RASNDGGGTNKKTFDNVAHLIEEAFSGYSNGNDHGASGD PITLTLKNGVTFAKDF SGRR-RASNDGGGTNKKTFDNVAHLIEEAFSGYSNGNDHGASGD PITLTLKNGVTFAKDFSGRR-RASNDGGGTNKKTFDNVAHLIEEAFSGYSNGNDHGASGD PITLTLKNGVTFAKDF SGRR-RASNDGGGTNKKTFDNVAHLIEEAF SGYSNGNDHGASGD PITLTLKNGVTFAKDFSGRR-RASNDGGGTNKKTFDNVAHLIEEAFSGYSNGNDHGASGD PITLTLKNGVTFAKDFSKRRRRASGDSSGTNEKTFDNVAHLIEEAF SGYSDGNDHGASDG PITLTLKNGVTFAKDFSKRRRRASGDSSGTNEKTFDNVAHLIEEAFSGYSDGNDHGASDG PITLTLKNGVTFAKDFSNRRRRASNDGEGTNKKTFDNVAHLIEEAFSGYSNGNDHRASDD PITLTLKNGVTFAKDF SKRRRRASGDSSGTNEKTFDNVAHLIEEAF SGYSDGNDHGASDG PITLTLKNGVTFAKDFSKRRRRASGDSSGTNEKTFDNVAHLIEEAFSGYSDGNDHGASDG $* * * * * * * * * * * * * * * * * * * * * * . * * *: * * * * * * * * * * * * * * * * * *: * * * * * *$

GHVKSPYYLLVLAQAVNNTEGENYLKTALQH IFGPNWNDLTTTNNDTTINDKWNQLKLKW GHVKSPYYLLVLAOAVNNTEGENYLKTALOH IFGPNWNDLTTTNNDTTINDKWNOLKLKW GHVKSPYYLLVLAQAVNNTEGENYLKTALOH IFGPNWNDLTTTNNDTTINDKWNOLKLKW GHVKSPYYLLVLAQAVNNTEGENYLKTALQH IFGPNWNDLTTTNNDTTINDKWNQLKLKW GHVKSPYYLLVLAQAVNNTEGENYLKTALOH IFGPNWNDLTTTNNDTTINDKWNOLKLKW GHVKSPYYLLVLAQAVNNTEGENYLKTALQH IFGPNWNDLTTTNNDTTINDKWNQLKLKW GHVKSPYYLLVLAQAVNNTEGENYLKTALQH IFGPNWNDLTTTNNGTTINDKWNQLKLKW GHVKSPYYLLVLAQAVNNTEGENYLKTALOH IFGPNWNDLTTTNNGTTINDKWNQLKLKW GHVKSPYYLLVLAQAVNNTEGENYLKTALQHIFGPNWNDLTTTNNDTTINDKWNQLKLKW GHVKSPYYLLVLAQAVNNTEGENYLKTALQH IFGPNWNDLTTTNNGTTINDKWNQLKLKW GHVKSPYYLLVLAQAVNNTEGENYLKTALQH IFGPNWNDLTTTNNGTTINDKWNQLKLKW

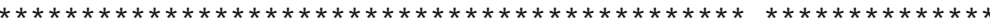

EKFKNNGENNHS INLNIYPADEGVEKAKIFAGVLRNGTNGVEDKVYQELNDKAKKEYEDK EKFKNNGENNHS INLNIYPADEGVEKAKIFAGVLRNGTNGVEDKVYQELNDKAKKEYEDK EKFKNNGENNHS INLNIYPADEGVEKAKIFAGVLRNGTNGVEDKVYQELNDKAKKEYEDK EKFKNNGENNHS INLNIYPADEGVEKAKIFAGVLRNGTNGVEDKVYQELNDKAKKEYEDK EKFKNNGENNHS INLNIYPADEGVEKAKIFAGVLRNGTNGVEDKVYQELNDKAKKEYEDK EKFKNNGENNHS INLNIYPADEGVEKAKIFAGVLRNGTNGVEDKVYQELNDKAKKEYEDK EKFKNNGENNHS INLNIYPADEGVEKAKIFAGVLRNGTNGVEDKVYQELNDKAKKEYEDK EKFKNNGENNHS INLNIYPADEGVEKAKIFAGVLRNGTNGVEDKVYQELNDKAKKEYEDK EKFKNNGENNHS INLNIYPADEGVEKAKIFAGVLRNGTNGVEDKVYQELNDKAKKEYEDK EKFKNNGENNHS INLNIYPADEGVEKAKIFAGVLRNGTNGVEDKVYQELNDKAKKEYEDK EKFKNNGENNHS INLNIYPADEGVEKAKIFAGVLRNGTNGVEDKVYQELNDKAKKEYEDK $* * * * * * * * * * * * * * * * * * * * * * * * * * * * * * * * * * * * * * * * * * * * * * * * * * * * * * * * * * * *$

FAKKFQGRFKSRFQNGEFDWAGDWAKEGNESYGSKETEEKYNGIKKEHTVNIGKHEIKVP FAKKFQGRFKSRF QNGEFDWAGDWAKEGNESYGSKETEEKYNGIKKEHTVNIGKHEIKVP FAKKFQGRFKSRFQNGEFDWAGDWAKEGNESYGSKETEEKYNGIKKEHTVNIGKHEIKVP FAKKFQGRFKSRFQNGEFDWAGDWAKEGNESYGSKETEEKYNGIKKEHTVNIGKHEIKVP FAKKFQGRFKSRFQNGEFDWAGDWAKEGNESYGSKETEEKYNGIKKEHTVNIGKHEIKVP FAKKFOGRFKSRFONGEFDWAGDWAKEGNESYGSKETEEKYNGIKKEHTVNIGKHEIKVP FAKKFQGRFKSRFQNGEFDWAGDWAKEGNESYGSKETEEKYNGIKKEHTVNIGKHEIKVP FAKKFQGRFKSRFQNGEFDWAGDWAKEGNESYGSKETEEKYNGIKKEHTVNIGKHEIKVP FAKKFQGRFKSRFQNGEFDWAGDWAKEGNESYGSKETEEKYNGIKKEHTVNIGKHEIKVP FAKKFQGRFKSRFQNGEFDWAGDWAKEGNESYGSKETEEKYNGIKKEHTVNIGKHEIKVP FAKKFQGRFKSRF QNGEFDWAGDWAKEGNESYGSKETEEKYNGIKKEHTVNIGKHEIKVP $* * * * * * * * * * * * * * * * * * * * * * * * * * * * * * * * * * * * * * * * * * * * * * * * * * * * * * * * * * * *$

TVSFENLNNINHQQDKSDGIDKSI ISELLAQP IYVAKADVPDVDPRVAQNDKAVDEDGLY TVSFENLNNINHOODKSDGIDKSI ISELLAOP IYVAKADVPDVDPRVAONDKAVDEDGLY TVSFENLNNINHQQDKSDGIDKSI ISELLAQP IYVAKADVPDVDPRVAQNDKAVDEDGLY TVSFENLNNINHQQDKSDGIDKS I ISELLAQP IYVAKADVPDVDPRVAQNDKAVDEDGLY TVSFENLNNINHQQDKSDGIDKSI ISELLAQPIYVAKADVPDVDPRVAQNDKAVDEDGLY TVSFENLNNINHQQDKSDGIDKSI ISELLAQPIYVAKADVPDVDPRVAQNDKAVDEDGLY 
$82-029362$

6644

HD 183

HMC 46

HMC 56

$35000 \mathrm{HP}$

NZS 1

NZS2

NZS 3

NZS 4

NZV1

82-029362

6644

HD 183

HMC 46

HMC 56

$35000 \mathrm{HP}$

NZS 1

NZS2

NZS 3

NZS 4

NZV1

82-029362

6644

HD 183

HMC 46

HMC 56

$35000 \mathrm{HP}$

NZ 1

NZS2

NZS 3

NZS 4

NZV1

82-029362

6644

HD 183

HMC 46

HMC 56

$35000 \mathrm{HP}$

NZS 1

NZS2

NZS3

NZS4

NZV1

82-029362

6644

HD 183

HMC 46

HMC 56

$35000 \mathrm{HP}$

NZS1

NZS2

NZS 3

NZS 4

NZV1

82-029362

6644

HD 183

HMC 46

HMC 56

$35000 \mathrm{HP}$
TVSFENLNNINHQQDKSDGIDKSI ISELLAQPIYVAKADVPDVDPRVAQNDKAVDEDGLY TVSFENLNNINHOODKSDGIDKSI ISELLAOP IYVAKADVPDVDPRVAONDKAVDEDGLY TVSFENLNNINHQQDKSDGIDKSI ISELLAQP IYVAKADVPDVDPRVAQNDKAVDEDGLY TVSFENLNNINHQQDKSDGIDKS I ISELLAQP IYVAKADVPDVDPRVAONDKAVDEDGLY TVSFENLNNINHQQDKSDGIDKSI ISELLAQP IYVAKADVPDVDPRVAQNDKAVDEDGLY $\star * * * * * * * * * * * * * * * * * * * * * * * * * * * * * * * * * * * * * * * * * * * * * * * * * * * * * * * * * * * * * * * * * * *$

RTRLSYINQNNYLGAKYFFNQLDTEDDKLKGIKRIGDNYFEHQLITRLIEKVADNHLTLK RTRLSY INQNNYLGAKYFFNQLDTEDDKLKGIKRIGDNYFEHQLITRLIEKVADNHLTLK RTRLSYINQNNYLGAKYFFNQLDTEDDKLKGIKRIGDNYFEHQLITRLIEKVADNHLTLK RTRLSY INONNYLGAKYFFNOLDTEDDKLKGIKRIGDNYFEHOLITRLIEKVADNHLTLK RTRLSYINQNNYLGAKYFFNQLDTEDDKLKGIKRIGDNYFEHQLITRLIEKVADNHLTLK RTRLSYINQNNYLGAKYFFNQLDTEDDKLKGIKRIGDNYFEHOLITRLIEKVADNHLTLK RTRLSYINQNNYLGAKYFFNQLDTEDDKLKGIKRIGDNYFEYQLITRLIEKVADNHLTLK RTRLSY INQNNYLGAKYFFNQLDTEDDKLKGIKRIGDNYFEYQLITRLIEKVADNHLTLK RTRLSYINQNNYLGAKYFFNQLDTEDDKLKGIKRIGDNYFEHQLITRLIEKVADNHLTLK RTRLSYINQNNYLGAKYFFNOLDTEDDKLKGIKRIGDNYFEYQLITRLIEKVADNHLTLK RTRLSYINQNNYLGAKYFFNQLDTEDDKLKGIKRIGDNYFEYQLITRLIEKVADNHLTLK $\star * * * * * * * * * * * * * * * * * * * * * * * * * * * * * * * * * * * * * * * * * * * * *: * * * * * * * * * * * * * * * * * * * *$

HGLHDIALVKKLIDSASIOAKDLNLKVGEALTKEOKDNLKEDIVWYVKTEVNGOEVLVPO HGLHDIALVKKLIDSAS IQAKDLNLKVGEALTKEQKDNLKEDIVWYVKTEVNGQEVLVPQ HGLHDIALVKKLIDSASIQAKDLNLKVGEALTKEQKDNLKEDIVWYVKTEVNGQEVLVPQ HGLHDIALVKKLIDSASIQAKDLNLKVGEALTKEQKDNLKEDIVWYVKTEVNGQEVLVPQ HGLHDIALVKKLIDSAS IQAKDLNLKVGEALTKEQKDNLKEDIVWYVKTEVNGQEVLVPQ HGLHDIALVKKLIDSASIQAKDLNLKVGEALTKEQKDNLKEDIVWYVKTEVNGQEVLVPQ HGLHDIALVKKLIDSASIQAKDLNLKVGEALTKEQKDNLKEDIVWYVKTEVNGQEVLVPQ HGLHDIALVKKLIDSASIOAKDLNLKVGEALTKEOKDNLKEDIVWYVKTEVNGOEVLVPO HGLHDIALVKKLIDSASIQAKDLNLKVGEALTKEQKDNLKEDIVWYVKTEVNGQEVLVPQ HGLHDIALVKKLIDSAS IQAKDLNLKVGEALTKEQKDNLKEDIVWYVKTEVNGQEVLVPQ HGLHDIALVKKLIDSASIQAKDLNLKVGEALTKEQKDNLKEDIVWYVKTEVNGQEVLVPQ $* * * * * * * * * * * * * * * * * * * * * * * * * * * * * * * * * * * * * * * * * * * * * * * * * * * * * * * * * * * *$

VYLAKQTIEEVEKQRGVGTGQIRAGI IDVKVDDVRNTGTIAGYAVGLEAKNKLKNTGDIL VYLAKQT IEEVEKQRGVGTGQIRAGI IDVKVDDVRNTGTIAGYAVGLEAKNKLKNTGDIL VYLAKQTIEEVEKQRGVGTGQIRAGI IDVKVDDVRNTGTIAGYAVGLEAKNKLKNTGDIL VYLAKQTIEEVEKQRGVGTGQIRAGIIDVKVDDVRNTGTIAGYAVGLEAKNKLKNTGDIL VYLAKQTIEEVEKQRGVGTGQIRAGI IDVKVDDVRNTGTIAGYAVGLEAKNKLKNTGDIL VYLAKQTIEEVEKQRGVGTGQIRAGI IDVKVDDVRNTGTIAGYAVGLEAKNKLKNTGDIL VYLAKOTIEEVEKORGVGTGOIRAGI IDVKVDDVRNTGTIAGYAVGLEAKNKLKNTGDIL VYLAKQTIEEVEKQRGVGTGQIRAGI IDVKVDDVRNTGTIAGYAVGLEAKNKLKNTGDIL VYLAKQTIEEVEKQRGVGTGQIRAGI IDVKVDDVRNTGTIAGYAVGLEAKNKLKNTGDIL VYLAKQTIEEVEKORGVGTGOIRAGIIDVKVDDVRNTGTIAGYAVGLEAKNKLKNTGDIL VYLAKQTIEEVEKQRGVGTGQIRAGI IDVKVDDVRNTGTIAGYAVGLEAKNKLKNTGDIL $* * * * * * * * * * * * * * * * * * * * * * * * * * * * * * * * * * * * * * * * * * * * * * * * * * * * * * * * * * * * * *$

SORLSKLVGKKGLESTGVTYVDETGATKVRKARIKSEGHIYLETDKDKNVDLTASELKGN SQRLSKLVGKKGLESTGVTYVDETGATKVRKARIKSEGHIYLETDKDKNVDLTASELKGN SQRLSKLVGKKGLESTGVTYVDETGATKVRKARIKSEGH I YLETDKDKNVDLTASELKGN SQRLSKLVGKKGLESTGVTYVDETGATKVRKARIKSEGHI YLETDKDKNVDLTASELKGN SQRLSKLVGKKGLESTGVTYVDETGATKVRKARIKSEGHIYLETDKDKNVDLTASELKGN SQRLSKLVGKKGLESTGVTYVDETGATKVRKARIKSEGHIYLETDKDKNVDLTASELKGN SQRLSKLVGKKGLESTGVTYVDETGATKVRKARIKSEGHIYLETDKDKNVDLTASELKGS SQRLSKLVGKKGLESTGVTYVDETGATKVRKARIKSEGHIYLETDKDKNVDLTASELKGS SQRLSKLVGKKGLESTGVTYVDETGATKVRKARIKSEGHIYLETDKDKNVDLTASELKGN SQRLSKLVGKKGLESTGVTYVDETGATKVRKARIKSEGHIYLETDKDKNVDLTASELKGS SQRLSKLVGKKGLESTGVTYVDETGATKVRKARIKSEGHIYLETDKDKNVDLTASELKGS $* * * * * * * * * * * * * * * * * * * * * * * * * * * * * * * * * * * * * * * * * * * * * * * * * * * * * * * * * * *$.

TGQIKAKDLNLNDIYETSYKYKYEKLFGKNGGEIGDRVTOTSOAKSVGTDASFDHLHLSL TGQIKAKDLNLNDIYETSYKYKYEKLFGKNGGEIGDRVTQTSQAKSVGTDASFDHLHLSL TGQIKAKDLNLNDIYETSYKYKYEKLFGKNGGEIGDRVTQTSQAKSVGTDASFDHLHLSL TGQIKAKDLNLNDIYETSYKYKYEKLFGKNGGEIGDRVTQTSQAKSVGTDASFDHLHLSL TGQIKAKDLNLNDIYETSYKYKYEKLFGKNGGEIGDRVTQTSQAKSVGTDASFDHLHLSL TGQIKAKDLNLNDIYETSYKYKYEKLFGKNGGEIGDRVTQTSQAKSVGTDASFDHLHLSL TGQIKAKDLNLNDIYETSYKYKYEKLFGKNGGEIGDRVTQTSQAKSVGTDASFDHLHLSL TGQIKAKDLNLNDIYETSYKYKYEKLFGKNGGEIGDRVTQTSQAKSVGTDASFDHLHLSL TGQIKAKDLNLNDIYETSYKYKYEKLFGKNGGEIGDRVTQTSQAKSVGTDASFDHLHLSL TGOIKAKDLNLNDIYETSYKYKYEKLFGKNGGEIGDRVTOTSOAKSVGTDASFDHLHLSL TGQIKAKDLNLNDIYETSYKYKYEKLFGKNGGEIGDRVTQTSQAKSVGTDASFDHLHLSI $* * * * * * * * * * * * * * * * * * * * * * * * * * * * * * * * * * * * * * * * * * * * * * * * * * * * * * * * * * * *$

EGDVNQTGSNLKANRTTGVVKGDFNTKAGKDLFHRQIDTVTSGTVYSASASGGGQSAGIS 
NZS1

NZS2

NZS 3

NZS 4

NZV1

82-029362

6644

HD 183

HMC 46

HMC 56

$35000 \mathrm{HP}$

NZS 1

NZS2

NZS3

NZS4

NZV1

82-029362

6644

HD 183

HMC 46

HMC 56

$35000 \mathrm{HP}$

NZS 1

NZS2

NZS 3

NZS4

NZV1

82-029362

6644

HD 183

HMC 46

HMC 56

$35000 \mathrm{HP}$

NZS 1

NZS2

NZS 3

NZS 4

NZV1

82-029362

6644

HD 183

HMC 46

HMC 56

$35000 \mathrm{HP}$

NZS1

NZS2

NZS3

NZS4

NZV1

82-029362

6644

HD 183

HMC 46

HMC 56

$35000 \mathrm{HP}$

NZS 1

NZS2

NZS 3

NZS 4

NZV1

82-029362

6644

HD 183
EGDVNQTGSNLKANRTTGVVKGDFNTKAGKDLFHRQIDTVTSGTVYSASASGGGQSAGIS EGDVNQTGSNLKANRTTGVVKGDFNTKAGKDLFHRQIDTVTSGTVYSASASGGGQSAGIS EGDVNQTGSNLKANRTTGVVKGDFNTKAGKDLFHRQIDTVTSGTVYSASASGGGQSAGIS EGDVNQTGSNLKANRTTGVVKGDFNTKAGKDLFHRQIDTVTSGTVYSASASGGGQSAGIS EGDVNQTGSNLKANRTTGVVKGDFNTKAGKDLFHRQIDTVTSGTVYSASASGGGQSAGIS EGDVNQTGSNLKANRTTGVVKGDFNTKAGKDLFHRQIDTVTSGTVYSASASGGGQSAGIS EGDVNOTGSNLKANRTTGVVKGDFNTKAGKDLFHROIDTVTSGTVYSASASGGGOSAGIS EGDVNQTGSNLKANRTTGVVKGDFNTKAGKDLFHRQIDTVTSGTVYSASASGGGQSAGIS EGDVNQTGSNLKANRTTGVVKGDFNTKAGKDLFHRQIDTVTSGTVYSASASGGGQSAGIS EGDVNQTGSNLKANRTTGVVKGDFNTKAGKDLFHRQIDTVTSGTVYSASASGGGQSAGIS $* * * * * * * * * * * * * * * * * * * * * * * * * * * * * * * * * * * * * * * * * * * * * * * * * * * * * * * * * * * *$

LTDQGVETYTNKTATAGANADVTNFMKRTRETETSLTHRNSEFNALSGELYVMGKADIGG LTDOGVETYTNKTATAGANADVTNFMKRTRETETSLTHRNSEFNALSGELYVMGKADIGG LTDQGVETYTNKTATAGANADVTNFMKRTRETETSLTHRNSEFNALSGELYVMGKADIGG LTDQGVETYTNKTATAGANADVTNFMKRTRETETSLTHRNSEFNALSGELYVMGKADIGG LTDQGVETYTNKTATAGANADVTNFMKRTRETETSLTHRNSEFNALSGELYVMGKADIGG LTDQGVETYTNKTATAGANADVTNFMKRTRETETSLTHRNSEFNALSGELYVMGKADIGG LTDQGVETYTNKTATAGANADVTNFMKRTRETETSLTHRNSEFNALSGELYVMGKADIGG LTDQGVETYTNKTATAGANADVTNFMKRTRETETSLTHRNSEFNALSGELYVMGKADIGG LTDQGVETYTNKTATAGANADVTNFMKRTRETETSLTHRNSEFNALSGELYVMGKADIGG LTDQGVETYTNKTATAGANADVTNFMKRTRETETSLTHRNSEFNALSGELYVMGKADIGG LTDQGVETYTNKTATAGANADVTNFMKRTRETETSLTHRNSEFNALSGELYVMGKADIGG $* * * * * * * * * * * * * * * * * * * * * * * * * * * * * * * * * * * * * * * * * * * * * * * * * * * * * * * * * * * *$

VDINRDVEVIKTPEEIAAEQKAAEEAKKAEVKENEASETAAKETEEAENDNVAEKDKTKP VDINRDVEVIKTPEEIAAEQKAAEEAKKAEVKENEASETAAKETEEAENDNVAEKDKTKP VDINRDVEVIKTPEEIAAEOKAAEEAKKAEVKENEASETAAKETEEAENDNVAEKDKTKP VDINRDVEVIKTPEEIAAEQKAAEEAKKAEVKENEASETAAKETEEAENDNVAEKDKTKP VDINRDVEVIKTPEEIAAEQKAAEEAKKAEVKENEASETAAKETEEAENDNVAEKDKTKP VDINRDVEVIKTPEEIAAEOKAAEEAKKAEVKENEASETAAKETEEAENDNVAEKDKTKP VDINRDVEVIKTPEEIAAEQKAAEEAKKAEVKENEASETAAKETEEAENDNVAEKDKTKP VDINRDVEVIKTPEE IAAEQKAAEEAKKAEVKENEASETAAKETEEAENDNVAEKDKTKP VDINRDVEVIKTPEEIAAEQKAAEEAKKAEVKENEASETAAKETEEAENDNVAEKDKTKP VDINRDVEVIKTPEEIAAEQKAAEEAKKAEVKENEASETAAKETEEAENDNVAEKDKTKP VDINRDVEVIKTPEEIAAEQKAAEEAKKAEVKENEASETAAKETEEAENDNVAEKDKTKP $* * * * * * * * * * * * * * * * * * * * * * * * * * * * * * * * * * * * * * * * * * * * * * * * * * * * * * * * * * * *$

KFKKLTDEEIAAAFETKGEDFFAAYKAREEEDRKKGFTLSAEQIESTKARDEKETTYYEL KFKKLTDEEIAAAFETKGEDFFAAYKAREEEDRKKGFTLSAEOIESTKARDEKETTYYEL KFKKLTDEEIAAAFETKGEDFFAAYKAREEEDRKKGFTLSAEQIESTKARDEKETTYYEL KFKKLTDEEIAAAFETKGEDFFAAYKAREEEDRKKGFTLSAEQIESTKARDEKETTYYEL KFKKLTDEEIAAAFETKGEDFFAAYKAREEEDRKKGFTLSAEOIESTKARDEKETTYYEL KFKKLTDEEIAAAFETKGEDFFAAYKAREEEDRKKGFTLSAEQIESTKARDEKETTYYEL KFKKLTDEEIAAAFETKGEDFFAAYKAREEEDRKKGFTLSAEQIESTKARDEKETTYYEL KFKKLTDEEIAAAFETKGEDFFAAYKAREEEDRKKGFTLSAEQIESTKARDEKETTYYEL KFKKLTDEEIAAAFETKGEDFFAAYKAREEEDRKKGFTLSAEQIESTKARDEKETTYYEL KFKKLTDEEIAAAFETKGEDFFAAYKAREEEDRKKGFTLSAEQIESTKARDEKETTYYEL KFKKLTDEEIAAAFETKGEDFFAAYKAREEEDRKKGFTLSAEQIESTKARDEKETTYYEL $* * * * * * * * * * * * * * * * * * * * * * * * * * * * * * * * * * * * * * * * * * * * * * * * * * * * * * * * * * * *$

KVGVGAEAEAHSAAADAISNKARQI IDTQNGLKQDGTVALQEASDVLNLATGDLAGASAK KVGVGAEAEAHSAAADAISNKARQI IDTQNGLKQDGTVALQEASDVLNLATGDLAGASAK KVGVGAEAEAHSAAADAISNKARQI IDTQNGLKQDGTVALQEASDVLNLATGDLAGASAK KVGVGAEAEAHSAAADAISNKARQI IDTQNGLKQDGTVALQEASDVLNLATGDLAGASAK KVGVGAEAEAHSAAADAISNKARQI IDTQNGLKQDGTVALQEASDVLNLATGDLAGASAK KVGVGAEAEAHSAAADAISNKARQI IDTQNGLKQDGTVALQEASDVLNLATGDLAGASAK KVGVGAEAEAHSAAADAISNKARQI IDTQNGLKQDGTVALQEASDVLNLATGDLAGASAK KVGVGAEAEAHSAAADAISNKARQI IDTQNGLKQDGTVALQEASDVLNLATGDLAGASAK KVGVGAEAEAHSAAADAISNKARQI IDTQNGLKQDGTVALQEASDVLNLATGDLAGASAK KVGVGAEAEAHSAAADAISNKARQI IDTQNGLKQDGTVALQEASDVLNLATGDLAGASAK KVGVGAEAEAHSAAADAISNKARQI IDTQNGLKQDGTVALQEASDVLNLATGDLAGASAK $* * * * * * * * * * * * * * * * * * * * * * * * * * * * * * * * * * * * * * * * * * * * * * * * * * * * * * * * * * *$

LKFELSTIEKKSRGASDGRS ILGGRLNLAARGGDITLNNVETTENSHLSLKARDNVNVNS LKFELSTIEKKSRGASDGRS ILGGRLNLAARGGDITLNNVETTENSHLSLKARDNVNVNS LKFELSTIEKKSRGASDGRS ILGGRLNLAARGGDITLNNVETTENSHLSLKARDNVNVNS LKFELSTIEKKSRGASDGRS ILGGRLNLAARGGDITLNNVETTENSHLSLKARDNVNVNS LKFELSTIEKKSRGASDGRS ILGGRLNLAARGGD ITLNNVETTENSHLSLKARDNVNVNS LKFELSTIEKKSRGASDGRSILGGRLNLAARGGDITLNNVETTENSHLSLKARDNVNVNS LKFELSTIEKKSRGASDGRS ILGGRLNLAARGGDITLNNVETTENSHLSLKARDNVNVNS LKFELSTIEKKSRGASDGRS ILGGRLNLAARGGDITLNNVETTENSHLSLKARDNVNVNS LKFELSTIEKKSRGASDGRS ILGGRLNLAARGGDITLNNVETTENSHLSLKARDNVNVNS 
HMC 46

HMC 56

$35000 \mathrm{HP}$

NZS 1

NZS2

NZS 3

NZS 4

NZV1

82-029362

6644

HD 183

HMC 46

HMC 56

$35000 \mathrm{HP}$

NZS 1

NZS2

NZS 3

NZS4

NZV1

82-029362

6644

HD 183

HMC 46

HMC 56

$35000 \mathrm{HP}$

NZS1

NZS2

NZS 3

NZS 4

NZV1

82-029362

6644

HD 183

HMC 46

HMC 56

$35000 \mathrm{HP}$

NZS 1

NZS2

NZS 3

NZS 4

NZV1

82-029362

6644

HD 183

HMC 46

HMC 56

$35000 \mathrm{HP}$

NZS 1

NZS2

NZS3

NZS4

NZV1

$82-029362$

6644

HD183

HMC 46

HMC5 6

$35000 \mathrm{HP}$

NZS1

NZS2

NZS3
LKFELSTIEKKSRGASDGRS ILGGRLNLAARGGDITLNNVETTENSHLSLKARDNVNVNS LKFELSTIEKKSRGASDGRS ILGGRLNLAARGGDITLNNVETTENSHLSLKARDNVNVNS $* * * * * * * * * * * * * * * * * * * * * * * * * * * * * * * * * * * * * * * * * * * * * * * * * * * * * * * * * * * *$

GVTEQKDESNSQSLKVTAGASSGCGVMAGGCSAGVSAGVSGSYNESNTESTSHTNSLLRG GVTEQKDESNSQSLKVTAGASSGCGVMAGGCSAGVSAGVSGSYNESNTESTSHTNSLLRG GVTEQKDESNSQSLKVTAGASSGCGVMAGGCSAGVSAGVSGSYNESNTESTSHTNSLLRG GVTEQKDESNSQSLKVTAGASSGCGVMAGGCSAGVSAGVSGSYNESNTESTSHTNSLLRG GVTEQKDESNSQSLKVTAGASSGCGVMAGGCSAGVSAGVSGSYNESNTESTSHTNSLLRG GVTEQKDESNSQSLKVTAGASSGCGVMAGGCSAGVSAGVSGSYNESNTESTSHTNSLLRG GVTEOKDESNSOSLKVTAGASSGCGVMAGGCSAGVSAGVSGSYNESNTESTSHTNSLLRG GVTEQKDESNSQSLKVTAGASSGCGVMAGGCSAGVSAGVSGSYNESNTESTSHTNSLLRG GVTEQKDESNSQSLKVTAGASSGCGVMAGGCSAGVSAGVSGSYNESNTESTSHTNSLLRG GVTEQKDESNSQSLKVTAGASSGCGVMAGGCSAGVSAGVSGSYNESNTESTSHTNSLLRG GVTEQKDESNSQSLKVTAGASSGCGVMAGGCSAGVSAGVSGSYNESNTESTSHTNSLLRG

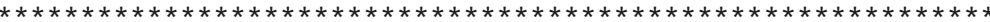

KSLRVEAGKDFNLISSNVDVDHLHLDVKGDTNVVSKQDSYSRKERGVNYSVSAGVGVSTA KSLRVEAGKDFNLISSNVDVDHLHLDVKGDTNVVSKODSYSRKERGVNYSVSAGVGVSTA KSLRVEAGKDFNLISSNVDVDHLHLDVKGDTNVVSKODSYSRKERGVNYSVSAGVGVSTA KSLRVEAGKDFNLISSNVDVDHLHLDVKGDTNVVSKODSYSRKERGVNYSVSAGVGVSTA KSLRVEAGKDFNLISSNVDVDHLHLDVKGDTNVVSKQDSYSRKERGVNYSVSAGVGVSTA KSLRVEAGKDFNLISSNVDVDHLHLDVKGDTNVVSKODSYSRKERGVNYSVSAGVGVSTA KSLRVEAGKDFNLISSNVDVDHLHLDVKGDTNVVSKQDSYSRKERGVNYSVSAGVGVSTA KSLRVEAGKDFNLISSNVDVDHLHLDVKGDTNVVSKODSYSRKERGVNYSVSAGVGVSTA KSLRVEAGKDFNLISSNVDVDHLHLDVKGDTNVVSKQDSYSRKERGVNYSVSAGVGVSTA KSLRVEAGKDFNLISSNVDVDHLHLDVKGDTNVVSKODSYSRKERGVNYSVSAGVGVSTA KSLRVEAGKDFNLISSNVDVDHLHLDVKGDTNVVSKQDSYSRKERGVNYSVSAGVGVSTA $* * * * * * * * * * * * * * * * * * * * * * * * * * * * * * * * * * * * * * * * * * * * * * * * * * * * * * * * * * * * * *$

GGARPNGSVGLGVSAENENSKIVKOQAGISAKRITGEINNLNLTGGY IENKGNPDELNVK GGARPNGSVGLGVSAENENSKIVKQQAGISAKRITGEINNLNLTGGYIENKGNPDELNVK GGARPNGSVGLGVSAENENSKIVKQOAGISAKRITGEINNLNLTGGY IENKGNPDELNVK GGARPNGSVGLGVSAENENSKIVKQQAGISAKRITGEINNLNLTGGY IENKGNPDELNVK GGARPNGSVGLGVSAENENSKIVKQQAGISAKRITGE INNLNLTGGY IENKGNPDELNVK GGARPNGSVGLGVSAENENSKIVKQQAGISAKRITGEINNLNLTGGY IENKGNPDELNVK GGARPNGSVGLGVSAENENSKIVKQQAGISAKRITGEINNLNLTGGY IENKGNPDELNVK GGARPNGSVGLGVSAENENSKIVKQQAGISAKRITGEINNLNLTGGYIENKGNPDELNVK GGARPNGSVGLGVSAENENSKIVKQQAGISAKRITGE INNLNLTGGY IENKGNPDELNVK GGARPNGSVGLGVSAENENSKIVKOOAGISAKRITGEINNLNLTGGY IENKGNPDELNVK GGARPNGSVGLGVSAENENSKIVKQQAGISAKRITGEINNLNLTGGY IENKGNPDELNVK $* * * * * * * * * * * * * * * * * * * * * * * * * * * * * * * * * * * * * * * * * * * * * * * * * * * * * * * * * * * * *$

GDITTHELKDEHHKDGGSFGGSVGVSETGVTQVNVNGGRVEQKHYEATOHSS ISGINTKG GDITTHELKDEHHKDGGSFGGSVGVSETGVTQVNVNGGRVEQKHYEATQHSS ISGINTKG GDITTHELKDEHHKDGGSFGGSVGVSETGVTQVNVNGGRVEQKHYEATQHSS ISGINTKG GDITTHELKDEHHKDGGSFGGSVGVSETGVTQVNVNGGRVEQKHYEATOHSS ISGINTKG GDITTHELKDEHHKDGGSFGGSVGVSETGVTQVNVNGGRVEQKHYEATQHSSISGINTKG GDITTHELKDEHHKDGGSFGGSVGVSETGVTQVNVNGGRVEQKHYEATQHSSISGINTKG GDITTHELKDEHHKDGGSFGGSVGVSETGVTQVNVNGGRVEQKHYEATQHSS ISGINTKG GDITTHELKDEHHKDGGSFGGSVGVSETGVTQVNVNGGRVEQKHYEATQHSSISGINTKG GDITTHELKDEHHKDGGSFGGSVGVSETGVTQVNVNGGRVEQKHYEATQHSS ISGINTKG GDITTHELKDEHHKDGGSFGGSVGVSETGVTQVNVNGGRVEQKHYEATQHSS ISGINTKG GDITTHELKDEHHKDGGSFGGSVGVSETGVTQVNVNGGRVEQKHYEATQHSS ISGINTKG $\star * * * * * * * * * * * * * * * * * * * * * * * * * * * * * * * * * * * * * * * * * * * * * * * * * * * * * * * * * * * *$

KTVGNFKTDRSOSTEVHRDDTIAATNFNFELGDIAELAKKGKEKWDNRSAKTTSSSODSA KTVGNFKTDRSQSTEVHRDDTIAATNFNFELGDIAELAKKGKEKWDNRSAKTTSSSQDSA KTVGNFKTDRSOSTEVHRDDTIAATNFNFELGDIAELAKKGKEKWDNRSAKTTSSSODSA KTVGNFKTDRSOSTEVHRDDTIAATNFNFELGDIAELAKKGKEKWDNRSAKTTSSSODSA KTVGNFKTDRSQSTEVHRDDTIAATNFNFELGDIAELAKKGKEKWDNRSAKTTSSSQDSA KTVGNFKTDRSQSTEVHRDDTIAATNFNFELGDIAELAKKGKEKWDNRSAKTTSSSQDSA KTVGNFKTDRSQSTEVHRDDTIAATNFNFELGDIAELAKKGKEKWDNRSAKTTSSSQDSA KTVGNFKTDRSOSTEVHRDDTIAATNFNFELGDIAELAKKGKEKWDNRSAKTTSSSQDSA KTVGNFKTDRSQSTEVHRDDTIAATNFNFELGDIAELAKKGKEKWDNRSAKTTSSSODSA KTVGNFKTDRSQSTEVHRDDTIAATNFNFELGDIAELAKKGKEKWDNRSAKTTSSSQDSA KTVGNFKTDRSOSTEVHRDDTIAATNFNFELGDIAELAKKGKEKWDNRSAKTTSSSODSA $* * * * * * * * * * * * * * * * * * * * * * * * * * * * * * * * * * * * * * * * * * * * * * * * * * * * * * * * * * * *$

HDPRSRSVENGYSELPRFKTADNDAGVDSPRLIKGEAEQAQTLALTKAGNDVIPEVQSLT HDPRSRSVENGYSELPRFKTADNDAGVDSPRLIKGEAEQAQTLALTKAGNDVIPEVQSLT HDPRSRSVENGYSELPRFKTADNDAGVDSPRLIKGEAEOAOTLALTKAGNDVIPEVOSLT HDPRSRSVENGYSELPRFKTADNDAGVDSPRLIKGEAEQAQTLALTKAGNDVIPEVQSLT 
NZS4

NZV1

$82-029362$

6644

HD 183

HMC 46

HMC 56

$35000 \mathrm{HP}$

NZS 1

NZS2

NZS 3

NZS 4

NZV1

82-029362

6644

HD 183

HMC 46

HMC 56

$35000 \mathrm{HP}$

NZS 1

NZS2

NZS 3

NZS4

NZV1

82-029362

6644

HD 183

HMC 46

HMC 56

$35000 \mathrm{HP}$

NZS 1

NZS2

NZS 3

NZS 4

NZV1

82-029362

6644

HD 183

HMC 46

HMC 56

$35000 \mathrm{HP}$

NZS1

NZS2

NZS 3

NZS 4

NZV1

82-029362

6644

HD183

HMC 46

HMC 56

$35000 \mathrm{HP}$

NZS 1

NZS2

NZS 3

NZS4

NZV1

$82-029362$

6644

HD 183

HMC 46

HMC5 6
HDPRSRSVENGYSELPRFKTADNDAGVDSPRLIKGEAEQAQTLALTKAGNDVIPEVQSLT HDPRSRSVENGYSELPRFKTADNDAGVDSPRLIKGEAEQAOTLALTKAGNDVIPEVQSLT HDPRSRSVENGYSELPRFKTADNDAGVDSPRLIKGEAEQAQTLALTKAGNDVIPEVQSLT HDPRSRSVENGYSELPRFKTADNDAGVDSPRLIKGEAEQAQTLALTKAGNDVIPEVQSLT HDPRSRSVENGYSELPRFKTADNDAGVDSPRLIKGEAEQAQTLALTKAGNDVIPEVQSLT HDPRSRSVENGYSELPRFKTADNDAGVDSPRLIKGEAEQAQTLALTKAGNDVIPEVQSLT HDPRSRSVENGYSELPRFKTADNDAGVDSPRLIKGEAEQAQTLALTKAGNDVIPEVQSLT $\star * * * * * * * * * * * * * * * * * * * * * * * * * * * * * * * * * * * * * * * * * * * * * * * * * * * * * * * * * * * * * * * * *$

QKARPQSLVDESPYAEIPALTRPQVKSNIAES IEVPQFRTKVSDGDEGNYAEITFPTNKA OKARPOSLVDESPYAEIPALTRPOVKSNIAES IEVPOFRTKVSDGDEGNYAEITFPTNKA QKARPQSLVDESPYAEIPALTRPQVKSNIAES IEVPQFRTKVSDGDEGNYAEITFPTNKA QKARPQSLVDESPYAEIPALTRPQVKSNIAES IEVPQFRTKVSDGDEGNYAEITFPTNKA QKARPQSLVDESPYAEIPALTRPQVKSNIAES IEVPQFRTKVSDGDEGNYAEITFPTNKA QKARPQSLVDESPYAE I PALTRPQVKSNIAES IEVPQFRTKVSDGDEGNYAEITFPTNKA OKARPQSLVDESPYAEIPALTRPOVKSNIAESIEVPQFRTKVSDGDEGNYAEITFPTNKA QKARPQSLVDESPYAE I PALTRPQVKSNIAES IEVPQFRTKVSDGDEGNYAEITFPTNKA QKARPQSLVDESPYAE I PALTRPQVKSNIAES IEVPQFRTKVSDGDEGNYAEITFPTNKA QKARPQSLVDESPYAEIPALTRPQVKSNIAESIEVPQFRTKVSDGDEGNYAEITFPTNKA QKARPQSLVDESPYAEIPALTRPQVKSNIAES IEVPQFRTKVSDGDEGNYAEITFPTNKA $* * * * * * * * * * * * * * * * * * * * * * * * * * * * * * * * * * * * * * * * * * * * * * * * * * * * * * * * * * * *$

AISSTQDVGDTPTPRALRLESESGYESAENLGLIPRGFKSSPKGEYEDISDAIEPQTRSR AISSTQDVGDTPTPRALRLESESGYESAENLGLIPRGFKSSPKGEYEDISDAIEPQTRSR AISSTQDVGDTPTPRALRLESESGYESAENLGLIPRGFKSSPKGEYEDISDAIEPQTRSR AISSTQDVGDTPTPRALRLESESGYESAENLGLIPRGFKSSPKGEYEDISDAIEPQTRSR AISSTQDVGDTPTPRALRLESESGYESAENLGLIPRGFKSSPKGEYEDISDAIEPQTRSR AISSTODVGDTPTPRALRLESESGYESAENLGLIPRGFKSSPKGEYEDISDAIEPOTRSR AISSTQDVGDTPTPRALRLESESGYESAENLGLIPRGFKSSPKGEYEDISDAIEPQTRSR AISSTQDVGDTPTPRALRLESESGYESAENLGLIPRGFKSSPKGEYEDISDAIEPQTRSR AISSTODVGDTPTPRALRLESESGYESAENLGLIPRGFKSSPKGEYEDISDAIEPQTRSR AISSTQDVGDTPTPRALRLESESGYESAENLGLIPRGFKSSPKGEYEDISDAIEPQTRSR AISSTQDVGDTPTPRALRLESESGYESAENLGLIPRGFKSSPKGEYEDISDAIEPQTRSR $* * * * * * * * * * * * * * * * * * * * * * * * * * * * * * * * * * * * * * * * * * * * * * * * * * * * * * * * * * * *$

KLDEPEPIYGTINKSPEAIARANAKADEAIQALGYDPRIKPVVPEEAPPALPPRNLQTKA KLDEPEPIYGTINKSPEAIARANAKADEAIQALGYDPRIKPVVPEEAPPALPPRNLQTKA KLDEPEPIYGTINKSPEAIARANAKADEAIOALGYDPRIKPVVPEEAPPALPPRNLOTKA KLDEPEPIYGTINKSPEAIARANAKADEAIQALGYDPRIKPVVPEEAPPALPPRNLQTKA KLDEPEPIYGTINKSPEAIARANAKADEAIOALGYDPRIKPVVPEEAPPALPPRNLOTKA KLDEPEPIYGTINKSPEAIARANAKADEAIQALGYDPRIKPVVPEEAPPALPPRNLQTKA KLDEPEPIYGTINKSPEAIARANAKADEAIQALGYDPRIKPVVPEEAPPALPPRNLQTKA KLDEPEPIYGTINKSPEAIARANAKADEAIOALGYDPRIKPVVPEEAPPALPPRNLOTKA KLDEPEPIYGTINKSPEAIARANAKADEAIQALGYDPRIKPVVPEEAPPALPPRNLQTKA KLDEPEPIYGTINKSPEAIARANAKADEAIOALGYDPRIKPVVPEEAPPALPPRNLOTKA KLDEPEPIYGTINKSPEAIARANAKADEAIQALGYDPRIKPVVPEEAPPALPPRNLQTKA $* * * * * * * * * * * * * * * * * * * * * * * * * * * * * * * * * * * * * * * * * * * * * * * * * * * * * * * * * * * * * * * *$

ISDYDDVSYVPDFKVRKTDEPEPIYGTINKSPEAIARANAKADEAIQASGYDPRIKPVVP ISDYDDVSYVPDFKVRKTDEPEPIYGTINKSPEAIARANAKADEAIQASGYDPRIKPVVP ISDYDDVSYVPDFKVRKTDEPEPIYGTINKSPEAIARANAKADEAIQASGYDPRIKPVVP ISDYDDVSYVPDFKVRKTDEPEPIYGTINKSPEAIARANAKADEAIQASGYDPRIKPVVP ISDYDDVSYVPDFKVRKTDEPEPIYGTINKSPEAIARANAKADEAIQASGYDPRIKPVVP ISDYDDVSYVPDFKVRKTDEPEPIYGTINKSPEAIARANAKADEAIQASGYDPRIKPVVP ISDYDDVSYVPDFKVRKTDEPEPIYGTINKSPEAIARANAKADEAIQASGYDPRIKPVVP ISDYDDVSYVPDFKVRKTDEPEPIYGTINKSPEAIARANAKADEAIQASGYDPRIKPVVP ISDYDDVSYVPDFKVRKTDEPEPIYGTINKSPEAIARANAKADEAIOASGYDPRIKPVVP ISDYDDVSYVPDFKVRKTDEPEPIYGTINKSPEAIARANAKADEAIQASGYDPRIKPVVP ISDYDDVSYVPDFKVRKTDEPEPIYGTINKSPEAIARANAKADEAIQASGYDPRIKPVVP $* * * * * * * * * * * * * * * * * * * * * * * * * * * * * * * * * * * * * * * * * * * * * * * * * * * * * * * * * * * * * * *$

EDAPPALPPRTOSLIDSTEVPSYRSALANVKFDDASPWPOPSALRSKAFADEPSSETPKS EDAPPALPPRTQSLIDSTEVPSYRSALANVKFDDASPWPQPSALRSKAFADEPSSETPKS EDAPPALPPRTOSLIDSTEVPSYRSALANVKFDDASPWPQPSALRSKAFADEPSSETPKS EDAPPALPPRTQSLIDSTEVPSYRSALANVKFDDASPWPQPSALRSKAFADEPSSETPKS EDAPPALPPRTQSLIDSTEVPSYRSALANVKFDDASPWPQPSALRSKAFADEPSSETPKS EDAPPALPPRTQSLIDSTEVPSYRSALANVKFDDASPWPQPSALRSKAFADEPSSETPKS EDAPPALPPRTQSLIDSTEVPSYRSALANVKFDDASPWPQPSALRSKAFADEPSSETPKS EDAPPALPPRTOSLIDSTEVPSYRSALANVKFDDASPWPOPSALRSKAFADEPSSETPKS EDAPPALPPRTQSLIDSTEVPSYRSALANVKFDDASPWPQPSALRSKAFADEPSSETPKS EDAPPALPPRTQSLIDSTEVPSYRSALANVKFDDASPWPQPSALRSKAFADEPSSETPKS EDAPPALPPRTQSLIDSTEVPSYRSALANVKFDDASPWPQPSALRSKAFADEPSSETPKS $* * * * * * * * * * * * * * * * * * * * * * * * * * * * * * * * * * * * * * * * * * * * * * * * * * * * * * * * * * * *$ 
$35000 \mathrm{HP}$

NZS 1

NZS2

NZS3

NZS 4

NZV1

82-029362

6644

HD 183

HMC 46

HMC 56

$35000 \mathrm{HP}$

NZS 1

NZS2

NZS 3

NZS 4

NZV1

82-029362

6644

HD 183

HMC 46

HMC 56

$35000 \mathrm{HP}$

NZS 1

NZS2

NZS3

NZS 4

NZV1

$82-029362$

6644

HD1 83

HMC 46

HMC 56

$35000 \mathrm{HP}$

NZS 1

NZS2

NZS 3

NZS 4

NZV1

82-029362

6644

HD 183

HMC 46

HMC 56

$35000 \mathrm{HP}$

NZS 1

NZS2

NZS 3

NZS 4

NZV1

82-029362

6644

HD 183

HMC 46

HMC 56

$35000 \mathrm{HP}$

NZS1

NZS2

NZS 3

NZS 4

NZV1

82-029362
RGKRGISEESLSSTVOPRSRKISEEDSSFERLPLRIIDNGSDYAEILPRNVKOTNEPATO RGKRGISEESLSSTVQPRSRKISEEDSSFERLPLRIIDNGSDYAEILPRNVKQTNEPATQ RGKRGISEESLSSTVOPRSRKISEEDSSFERLPLRI IDNGSDYAEILPRNVKQTNEPATQ RGKRGISEESLSSTVQPRSRKISEEDSSFERLPLRIIDNGSDYAEILPRNVKQTNEPATQ RGKRGISEESLSSTVQPRSRKISEEDSSFERLPLRI IDNGSDYAEILPRNVKQTNEPATQ RGKRGISEESLSSTVQPRSRKISEEDSSFERLPLRIIDNGSDYAEILPRNVKQTNEPATQ RGKRGISEESLSSTVQPRSRKISEEDSSFERLPLRI IDNGSDYAEILPRNVKQTNEPATQ RGKRGISEESLSSTVQPRSRKISEEDSSFERLPLRI IDNGSDYAEILPRNVKQTNEPATQ RGKRGISEESLSSTVQPRSRKISEEDSSFERLPLRI IDNGSDYAEILPRNVKQTNEPATQ RGKRGISEESLSSTVOPRSRKISEEDSSFERLPLRI IDNGSDYAE ILPRNVKOTNEPATQ RGKRGISEESLSSTVQPRSRKISEEDSSFERLPLRIIDNGSDYAEILPRNVKQTNEPATQ $\star * * * * * * * * * * * * * * * * * * * * * * * * * * * * * * * * * * * * * * * * * * * * * * * * * * * * * * * * * * * * * * *$

AIRAPKALDNNDVIAERPSFKLRQLDDDVESVNGIYSSIKPKALIEEGTPITRQVKTVQE AIRAPKALDNNDVIAERPSFKLRQLDDDVESVNGIYSSIKPKALIEEGTPITRQVKTVQE AIRAPKALDNNDVIAERPSFKLRQLDDDVESVNGIYSSIKPKALIEEGTPITRQVKTVQE AIRAPKALDNNDVIAERPSFKLRQLDDDVESVNGIYSSIKPKALIEEGTPITRQVKTVQE AIRAPKALDNNDVIAERPSFKLROLDDDVESVNGIYSSIKPKALIEEGTPITROVKTVOE AIRAPKALDNNDVIAERP SFKLRQLDDDVESVNGIYSS IKPKALIEEGTPITRQVKTVQE AIRAPKALDNNDVIAERPSFKLRQLDDDVESVNGIYSS IKPKALIEEGTP ITROVKTVQE AIRAPKALDNNDVIAERPSFKLRQLDDDVESVNGIYSSIKPKALIEEGTPITRQVKTVQE AIRAPKALDNNDVIAERPSFKLRQLDDDVESVNGIYSS IKPKALIEEGTPITRQVKTVQE AIRAPKALDNNDVIAERPSFKLRQLDDDVESVNGIYSS IKPKALIEEGTPITRQVKTVQE AIRAPKALDNNDVIAERPSFKLRQLDDDVESVNGIYSS IKPKALIEEGTPITRQVKTVQE $* * * * * * * * * * * * * * * * * * * * * * * * * * * * * * * * * * * * * * * * * * * * * * * * * * * * * * * * * * * *$

ETPVTDLVNKRELVKEDRSLLDKVQDTFOPLKVRSKINDVRSSVEEYGGEVTFKYAOSKG ETPVTNLVNKRELVKEDRSLLDKVQDTFQPLKVRSKINDVRSSVEEYGGEVTFKYAQSKG ETPVTNLVNKRELVKEDRSLLDKVQDTFQPLKVRSKINDVRSSVEEYGGEVTFKYAQSKG ETPVTNLVNKRELVKEDRSLLDKVODTFOPLKVRSKINDVRSSVEEYGGEVTFKYAOSKG ETPVTNLVNKRELVKEDRSLLDKVQDTFQPLKVRSKINDVRSSVEEYGGEVTFKYAQSKG ETPVTNLVNKRELVKEDRSLLDKVQDTFQPLKVRSKINDVRSSVEEYGGEVTFKYAQSKG ETPVTDLVNKRELVKEDRSLLDKVQDTFOPLKVRSKINDVRSSVEEYGGEVTFKYAQSKG ETPVTDLVNKRELVKEDRSLLDKVQDTFQPLKVRSKINDVRSSVEEYGGEVTFKYAQSKG ETPVTDLVNKRELVKEDRSLLDKVQDTFQPLKVRSKINDVRSSVEEYGGEVTFKYAQSKG ETPVTDLVNKRELVKEDRSLLDKVQDTFQPLKVRSKINDVRSSVEEYGGEVTFKYAQSKG ETPVTDLVNKRELVKEDRSLLDKVQDTFQPLKVRSKINDVRSSVEEYGGEVTFKYAQSKG $* * * * *: * * * * * * * * * * * * * * * * * * * * * * * * * * * * * * * * * * * * * * * * * * * * * * * * * * * * * *$

EVYNEIVKHAETQNGVCEATCSHWIAKKVNDENIWTDLYKDGQKGRKGGLNKDAIESIEK EVYNEIVKHAETQNGVCEATCSHWIAKKVNDENIWTDLYKDGQKGRKGGLNKDAIES IEK EVYNEIVKHAETONGVCEATCSHWIAKKVNDENIWTDLYKDGOKGRKGGLNKDAIESIEK EVYNE IVKHAETQNGVCEATCSHWIAKKVNDENIWTDLYKDGQKGRKGGLNKDAIES IEK EVYNE IVKHAETQNGVCEATCSHWIAKKVNDENIWTDLYKDGQKGRKGGLNKDAIESIEK EVYNE IVKHAETQNGVCEATCSHWIAKKVNDENIWTDLYKDGQKGRKGGLNKDAIESIEK EVYNE IVKHAETQNGVCEATCSHWIAKKVNDENIWTDLYKDGQKGRKGGLNKDAIESIEK EVYNE IVKHAETONGVCEATCSHWIAKKVNDENIWTDLYKDGOKGRKGGLNKDAIESIEK EVYNE IVKHAETQNGVCEATCSHWIAKKVNDENIWTDLYKDGQKGRKGGLNKDAIES IEK EVYNE IVKHAETQNGVCEATCSHWIAKKVNDENIWTDLYKDGQKGRKGGLNKDAIES IEK EVYNEIVKHAETQNGVCEATCSHWIAKKVNDENIWTDLYKDGQKGRKGGLNKDAIESIEK $* * * * * * * * * * * * * * * * * * * * * * * * * * * * * * * * * * * * * * * * * * * * * * * * * * * * * * * * * * * *$

LQTEF INAGTATQQFKLTNTWLEEQGVVPKOKYFGKLSRADEVAGTVSKNDVSALVKAIL LQTEF INAGTATQOFKLTNTWLEEQGVVPKOKYFGKLSRADEVAGTVSKNDVSALVKAIL LQTEF INAGTATQQFKLTNTWLEEQGVVPKQKYFGKLSRADEVAGTVSKNDVSALVKAIL LQTEF INAGTATQQFKLTNTWLEEQGVVPKQKYFGKLSRADEVAGTVSKNDVSALVKAIL LQTEF INAGTATQQFKLTNTWLEEQGVVPKQKYFGKLSRADEVAGTVSKNDVSALVKAIL LQTEF INAGTATQOFKLTNTWLEEQGVVPKOKYFGKLSRADEVAGTVSKNDVSALVKAIL LOTEF INAGTATOQFKLTNTWLEEOGVVPKOKYFGKLSRADEVAGTVSKNDVSALVKAIL LQTEF INAGTATQQFKLTNTWLEEQGVVPKOKYFGKLSRADEVAGTVSKNDVSALVKAIL LQTEF INAGTATQQFKLTNTWLEEQGVVPKQKYFGKLSRADEVAGTVSKNDVSALVKAIL LQTEF INAGTATQQFKLTNTWLEEQGVVPKQKYFGKLSRADEVAGTVSKNDVSALVKAIL LQTEF INAGTATQQFKLTNTWLEEQGVVPKQKYFGKLSRADEVAGTVSKNDVSALVKAIL $* * * * * * * * * * * * * * * * * * * * * * * * * * * * * * * * * * * * * * * * * * * * * * * * * * * * * * * * * * * * *$

DTGNESSAVKKIS INLEGGSHTVSAS IEGQKVVFFDPNFGE ITFKDKKSFEKWMKNAFWK DTGNESSAVKKIS INLEGGSHTVSAS IEGQKVVFFDPNFGE ITFKDKKSFEKWMKNAFWK DTGNESSAVKKIS INLEGGSHTVSAS IEGOKVVFFDPNFGEITFKDKKSFEKWMKNAFWK DTGNESSAVKK IS INLEGGSHTVSAS IEGQKVVFFDPNFGEITFKDKKSFEKWMKNAFWK DTGNESSAVKKIS INLEGGSHTVSAS IEGQKVVFFDPNFGE ITFKDKKSFEKWMKNAFWK DTGNESSAVKKISINLEGGSHTVSAS IEGOKVVFFDPNFGEITFKDKKSFEKWMKNAFWK DTGNESSAVKKIS INLEGGSHTVSAS IEGQKVVFFDPNFGEITFKDKKSFEKWMKNAFWK 
6644

HD 183

HMC 46

HMC 56

$35000 \mathrm{HP}$

NZS 1

NZS2

NZS 3

NZS 4

NZV1

82-029362

6644

HD 183

HMC 46

HMC 56

$35000 \mathrm{HP}$

NZS 1

NZS2

NZS 3

NZS 4

NZV1

82-029362

6644

HD 183

HMC 46

HMC 56

$35000 \mathrm{HP}$

NZS 1

NZS2

NZS 3

NZS 4

NZV1

82-029362

6644

HD 183

HMC 46

HMC 56

$35000 \mathrm{HP}$

NZ S 1

NZS2

NZS 3

NZS 4

NZV1

82-029362

6644

HD 183

HMC 46

HMC 56

$35000 \mathrm{HP}$

NZS 1

NZS2

NZS 3

NZS 4

NZV1

82-029362

6644

HD 183

HMC 46

HMC 56
DTGNESSAVKKIS INLEGGSHTVSASIEGQKVVFFDPNFGEITFKDKKSFEKWMKNAFWK DTGNESSAVKKIS INLEGGSHTVSASIEGOKVVFFDPNFGEITFKDKKSFEKWMKNAFWK DTGNESSAVKKIS INLEGGSHTVSAS IEGQKVVFFDPNFGEITFKDKKSFEKWMKNAFWK DTGNESSAVKKIS INLEGGSHTVSAS IEGQKVVFFDPNFGE ITFKDKKSFEKWMKNAFWK $* * * * * * * * * * * * * * * * * * * * * * * * * * * * * * * * * * * * * * * * * * * * * * * * * * * * * * * * * * * *$

KSGYAGKKDTKRFFNVVNYHKNSKRNKVIDVNQNHIQQLAGSEGFSPSLPTRPQLANAAG KSGYAGKKDTKRFFNVVNYHKNSKRNKVIDVNQNHIQQLAGSEGF SPSLPTRPQLANAAG KSGYAGKKDTKRFFNVVNYHKNSKRNKVIDVNONHIOOLAGSEGFSPSLPTRPOLANAAG KSGYAGKKDTKRFFNVVNYHKNSKRNKVIDVNQNHIQQLAGSEGF SPSLPTRPQLANAAG KSGYAGKKDTKRFFNVVNYHKNSKRNKVIDVNONHIOOLAGSEGF SPSLPTRPOLANAAG KSGYAGKKDTKRFFNVVNYHKNSKRNKVIDVNONH IQQLAGSEGFSPSLPTRPQLANAAG KSGYAGKKDTKRFFNVVNYHKNSKRNKVIDVNQNHIQQLAGSEGF SPSLPTRPQLANAAG KSGYAGKKDTKRFFNVVNYHKNSKRNKVIDVNONH IOQLAGSEGFSPSLPTRPOLANAAG KSGYAGKKDTKRF FNVVNYHKNSKRNKVIDVNQNHIQQLAGSEGFSPSLPTRPQLANAAG KSGYAGKKDTKRFFNVVNYHKNSKRNKVIDVNONH IOOLAGSEGFSPSLPTRPOLANAAG KSGYAGKKDTKRFFNVVNYHKNSKRNKVIDVNQNHIQQLAGSEGFSPSLPTRPQLANAAG $\star * * * * * * * * * * * * * * * * * * * * * * * * * * * * * * * * * * * * * * * * * * * * * * * * * * * * * * * * * * * * * * * *$

IKSNEMSSLFSWSKLKHLF SRESGKKAQVEGPEIKHLGGVVDKDAFYFPLDKIVTRRDAE IKSNEMSSLFSWSKLKHLF SRESGKKAQVEGPEIKHLGGVVDKDAFYFPLDKIVTRRDAE IKSNEMSSLF SWSKLKHLF SRESGKKAQVEGPEIKHLGGVVDKDAFYFPLDKIVTRRDAE IKSNEMSSLFSWSKLKHLF SRESGKKAOVEGPEIKHLGGVVDKDAFYFPLDKIVTRRDAE IKSNEMSSLFSWSKLKHLF SRESGKKAQVEGPEIKHLGGVVDKDAFYFPLDKIVTRRDAE IKSNEMSSLF SWSKLKHLF SRESGKKAQVEGPEIKHLGGVVDKDAFYFPLDKIVTRRDAE IKSNEMSSLFSWSKLKHLF SRESGKKAQVEGPEIKHLGGVVDKDAFYFPLDKIVTRRDAE IKSNEMSSLF SWSKLKHLF SRESGKKAQVEGPEIKHLGGVVDKDAFYFPLDKIVTRRDAE IKSNEMSSLFSWSKLKHLF SRESGKKAOVEGPEIKHLGGVVDKDAFYFPLDKIVTRRDAE IKSNEMSSLF SWSKLKHLF SRESGKKAQVEGPEIKHLGGVVDKDAFYFPLDKIVTRRDAE IKSNEMSSLFSWSKLKHLF SRESGKKAQVEGPEIKHLGGVVDKDAFYFPLDKIVTRRDAE $* * * * * * * * * * * * * * * * * * * * * * * * * * * * * * * * * * * * * * * * * * * * * * * * * * * * * * * * * * * * * * * * *$

GEIRVNMDNIKKAFNPRDKHYNSOEARSLRSLYNODPSMSGTRFIIENOVIANPFSSADI GE IRVNMDNIKKAFNPRDKHYNSQEARSLRSLYNQDPSMSGTRF I IENQVIANPFSSADL GEIRVNMDNIKKAFNPRDKHYNSOEARSLRSLYNQDPSMSGTRF I IENQVIANPFSSADI GE IRVNMDNIKKAFNPRDKHYNSQEARSLRSLYNQDPSMSGTRF I IENQVIANPFSSADL GEIRVNMDNIKKAFNPRDKHYNSQEARSLRSLYNQDPSMSGTRF I IENQVIANPF SSADI GEIRVNMDNI KKAFNPRDKHYNSOEARSLRSLYNODPSMSGTRF I IENOVIANPF SSADI GEIRVNMDNIKKAFNPRDKHYNSQEARSLRSLYNQDPSMSGTRF I IENQVIANPFSSADL GEIRVNMDNIKKAFNPRDKHYNSOEARSLRSLYNODPSMSGTRF I IENOVIANPF SSADI GEIRVNMDNIKKAFNPRDKHYNSQEARSLRSLYNQDPSMSGTRFIIENQVIANPFSSADI GEIRVNMDNIKKAFNPRDKHYNSQEARSLRSLYNQDPSMSGTRFIIENQVIANPFSSADI GEIRVNMDNI KKAFNPRDKHYNSOEARSLRSLYNODPSMSGTRF I IENOVIANPFSSADI $* * * * * * * * * * * * * * * * * * * * * * * * * * * * * * * * * * * * * * * * * * * * * * * * * * * * * * * * * * * *$

QSYIQAQQSKLPELGRQARRALPELPTAANKGRGSRVEEQNIVTRPRVEDVYATVNKGAK QSYIQAQQSKLPELGRQARRALPELPTAANKGRGSRVEEQNIVTRPRVEDVYATVNKGAK QSYIQAQQSKLPELGRQARRALPELPTAANKGRGSRVEEQNIVTRPRVEDVYATVNKGAK QSYIQAQQSKLPELGRQARRALPELPTAANKGRGSRVEEQNIVTRPRVEDVYATVNKGAK QSYIOAOQSKLPELGROARRALPELPTAANKGRGSRVEEONIVTRPRVEDVYATVNKGAK QSYIQAQQSKLPELGRQARRALPELPTAANKGRGSRVEEQNIVTRPRVEDVYATVNKGAK OSYIOAOOSKLPELGROARRALPELPTAANKGRGSRVEEONIVTRPRVEDVYATVNKGAK QSYIQAQQSKLPELGRQARRALPELPTAANKGRGSRVEEQNIVTRPRVEDVYATVNKGAK QSYIQAQQSKLPELGRQARRALPELPTAANKGRGSRVEEQNIVTRPRVEDVYATVNKGAK QSYIQAQQSKLPELGRQARRALPELPTAANKGRGSRVEEQNIVTRPRVEDVYATVNKGAK QSYIQAQQSKLPELGRQARRALPELPTAANKGRGSRVEEQNIVTRPRVEDVYATVNKGAK $* * * * * * * * * * * * * * * * * * * * * * * * * * * * * * * * * * * * * * * * * * * * * * * * * * * * * * * * * * * *$

HGEAQQPGSFYTKKLVDQVSHVPNTEPVYADLHFNRNGRVVRQTEPEVIYEKIRGQQVEV HGEAQQPGSFYTKKLVDQVSHVPNTEPVYADLHFNRNGRVVRQTEPEVIYEKIRGQQVEV HGEAQQPGSFYTKKLVDQVSHVPNTEPVYADLHFNRNGRVVRQTEPEVIYEKIRGQQVEV HGEAQQPGSFYTKKLVDQVSHVPNTEPVYADLHFNRNGRVVRQTEPEVIYEKIRGQQVEV HGEAQQPGSFYTKKLVDQVSHVPNTEPVYADLHFNRNGRVVRQTEPEVIYEKIRGQQVEV HGEAOQPGSFYTKKLVDOVSHVPNTEPVYADLHFNRNGRVVROTEPEVIYEKIRGOOVEV HGEAQQPGSFYTKKLVDQVSHVPTTEPVYADLHFNRNGRVVRQTEPEVIYEKIRGQQVEV HGEAQQPGSFYTKKLVDQVSHVPTTEPVYADLHFNRNGRVVRQTEPEVIYEKIRGQQVEV HGEAQQPGSFYTKKLVDQVSHVPNTEPVYADLHFNRNGRVVRQTEPEVIYEKIRGQQVEV HGEAQQPGSFYTKKLVDQVSHVPTTEPVYADLHFNRNGRVVRQTEPEVIYEKIRGQQVEV HGEAQQPGSFYTKKLVDQVSHVPTTEPVYADLHFNRNGRVVRQTEPEVIYEKIRGQQVEV $\star * * * * * * * * * * * * * * * * * * * * * * * * * . . * * * * * * * * * * * * * * * * * * * * * * * * * * * * * * * * * * * * * * * *$

DDPSSLYAKVNRNRRLDNVEGFYPPEOLRTRSDKLAEOVSRVPTTEPVYADLRFKSAEDD DDPSSLYAKVNRNRRLDNVEGFYPPEQLRTRSDKLAEQVSRVPTTEPVYADLRFKSAEDD 
NZS2

NZS 3

NZS4

NZV1

82-029362

6644

HD 183

HMC 46

HMC 56

$35000 \mathrm{HP}$

NZS 1

NZS2

NZS 3

NZS 4

NZV1

82-029362

6644

HD 183

HMC 46

HMC 56

$35000 \mathrm{HP}$

NZS 1

NZS2

NZS 3

NZS 4

NZV1

82-029362

6644

HD 183

HMC 46

HMC 56

$35000 \mathrm{HP}$

NZS 1

NZS2

NZS 3

NZS4

NZV1

82-029362

6644

HD 183

HMC 46

HMC 56

$35000 \mathrm{HP}$

NZS 1

NZS2

NZS 3

NZS 4

NZV1

82-029362

6644

HD 183

HMC 46

HMC 56

$35000 \mathrm{HP}$

NZS1

NZS2

NZS3

NZS 4

NZV1

82-029362

6644

HD 183

HMC 46
DDPSSLYAKVNRNRRLDNVEGFYPPEQLRTRSDKLAEQVSRVPTTEPVYADLRFKSAEDD DDPSSLYAKVNRNRRLDNVEGFYPPEQLRTRSDKLAEQVSRVPTTEPVYADLRFKSAEDD DDPSSLYAKVNRNRRLDNVEGFYPPEQLRTRSDKLAEQVSRVPTTEPVYADLRFKSAEDD DDPSSLYAKVNRNRRLDNVEGFYPPEOLRTRSDKLAEOVSRVPTTEPVYADLRFKSAEDD DDPSSLYAKVNRNRRLDNVEGFYPPEQLRTRSDKLAEQVSRVPTTEPVYADLRFKSAEDD DDPSSLYAKVNRNRRLDNVEGFYPPEQLRTRSDKLAEQVSRVPTTEPVYADLRFKSAEDD DDPSSLYAKVNRNRRLDNVEGFYPPEQLRTRSDKLAEQVSRVPTTEPVYADLRFKSAEDD DDPSSLYAKVNRNRRLDNVEGFYPPEQLRTRSDKLAEQVSRVPTTEPVYADLRFKSAEDD DDPSSLYAKVNRNRRLDNVEGFYPPEQLRTRSDKLAEQVSRVPTTEPVYADLRFKSAEDD $* * * * * * * * * * * * * * * * * * * * * * * * * * * * * * * * * * * * * * * * * * * * * * * * * * * * * * * * * * * *$

YAPALPARPELGNAAGFRKAKVKGEESESTWSRLKHLFSRESGKTKVEEVENEYKSQTNG YAPALPARPELGNAAGFRKAKVKGEESESTWSRLKHLFSRESGKTKVEEVENEYKSQTNG YAPALPARPELGNAAGFRKAKVKGEESESTWSRLKHLFSRESGKTKVEEVENEYKSQTNG YAPALPARPELGNAAGFRKAKVKGEESESTWSRLKHLFSRESGKTKVEEVENEYKSQTNG YAPALPARPELGNAAGFRKAKVKGEESESTWSRLKHLFSRESGKTKVEEVENEYKSQTNG YAPALPARPELGNAAGFRKAKVKGEESESTWSRLKHLFSRESGKTKVEEVENEYKSQTNG YAPALPARPELGNAAGFRKAKVKGEESESTWSRLKHLFSRESGKTKVEEVENEYKSQTNG YAPALPARPELGNAAGFRKAKVKGEESESTWSRLKHLFSRESGKTKVEEVENEYKSOTNG YAPALPARPELGNAAGFRKAKVKGEESESTWSRLKHLF SRESGKTKVEEVENEYKSQTNG YAPALPARPELGNAAGFRKAKVKGEESESTWSRLKHLFSRESGKTKVEEVENEYKSQTNG YAPALPARPELGNAAGFRKAKVKGEESESTWSRLKHLF SRESGKTKVEEVENEYKSOTNG $* * * * * * * * * * * * * * * * * * * * * * * * * * * * * * * * * * * * * * * * * * * * * * * * * * * * * * * * * * * *$

ENSLEIKTIEHIPTRLKNLESPREAOSDLGENALIYGLQRGROALISKANAADKEGKNAI ENSLEIKTIEHIPTRLKNLESPREAQSDLGENALIYGLQRGRQALISKANAADKEGKNAI ENSLEIKTIEHIPTRLKNLESPREAQSDLGENALIYGLQRGRQALISKANAADKEGKNAI ENSLEIKTIEH IPTRLKNLESPREAOSDLGENALIYGLORGROALISKANAADKEGKNAI ENSLEIKTIEH IPTRLKNLES PREAQSDLGENALIYGLQRGRQALISKANAADKEGKNAI ENSLEIKTIEH I PTRLKNLESPREAQSDLGENALIYGLQRGRQALISKANAADKEGKNAI ENSLEIKTIEHIPTRLKNLESPREAOSDLGENALIYGLQRGROALISKANAADKEGKNAI ENSLEIKTIEHIPTRLKNLESPREAQSDLGENALIYGLQRGRQALISKANAADKEGKNAI ENSLEIKTIEH IPTRLKNLESPREAQSDLGENAL I YGLQRGRQALISKANAADKEGKNAI ENSLE IKTIEH IPTRLKNLES PREAQSDLGENAL I YGLQRGRQALISKANAADKEGKNAI ENSLEIKTIEHIPTRLKNLESPREAQSDLGENALIYGLQRGRQALISKANAADKEGKNAI $* * * * * * * * * * * * * * * * * * * * * * * * * * * * * * * * * * * * * * * * * * * * * * * * * * * * * * * * * * * *$ t

LADSYIGKLNLGFEFGELTKFAKQVKDGKVTEQDIQNIASFNDETAKLARRSEPKNRIND LADSY IGKLNLGFEFGELTKFAKQVKDGKVTEQDIQNIASFNDETAKLARRSEPKNRIND LADSYIGKLNLGFEFGELTKFAKOVKDGKVTEODIONIASFNDETAKLARRSEPKNRIND LADSYIGKLNLGFEFGELTKFAKQVKDGKVTEQDIQNIASFNDETAKLARRSEPKNRIND LADSY IGKLNLGFEFGELTKFAKQVKDGKVTEQDIQNIASFNDETAKLARRSEPKNRIND LADSYIGKLNLGFEFGELTKFAKOVKDGKVTEODIONIASFNDETAKLARRSEPKNRIND LADSY I GKLNLGFEFGELTKFAKQVKDGKVTEQDIQNIASFNDETAKLARRSEPKNRIND LADSY IGKLNLGFEFGELTKFAKQVKDGKVTEQDIQNIASFNDETAKLARRSEPKNRIND LADSY I GKLNLGFEFGELTKFAKQVKDGKVTEQDIQNIASFNDETAKLARRSEPKNRIND LADSYIGKLNLGFEFGELTKFAKQVKDGKVTEQDIQNIASFNDETAKLARRSEPKNRIND LADSYIGKLNLGFEFGELTKFAKQVKDGKVTEQDIQNIASFNDETAKLARRSEPKNRIND $* * * * * * * * * * * * * * * * * * * * * * * * * * * * * * * * * * * * * * * * * * * * * * * * * * * * * * * * * * * *$

ANVDDNQRI IRELINNEAAVDALKRIATLSDQEKAMHSTLRANEKFDMDELEESPNYTTA ANVDDNQRI IREL INNEAAVDALKRIATLSDQEKAMHSTLRANEKF DMDELEESPNYTTA ANVDDNQRI IRELINNEAAVDALKRIATLSDQEKAMHSTLRANEKFDMDELEESPNYTTA ANVDDNQRI IRELINNEAAVDALKRIATLSDQEKAMHSTLRANEKFDMDELEESPNYTTA ANVDDNQRI IRELINNEAAVDALKRIATLSDQEKAMHSTLRANEKFDMDELEESPNYTTA ANVDDNQRI IRELINNEAAVDALKRIATLSDQEKAMHSTLRANEKFDMDELEESPNYTTA ANVDDNQRI IRELINNEAAVDALKRIATLSDQEKAMHSTLRANEKFDMDELEESPNYTTA ANVDDNQRI IRELINNEAAVDALKRIATLSDQEKAMHSTLRANEKFDMDELEESPNYTTA ANVDDNQRI IRELINNEAAVDALKRIATLSDQEKAMHSTLRANEKFDMDELEESPNYTTA ANVDDNQRI IRELINNEAAVDALKRIATLSDQEKAMHSTLRANEKF DMDELEESPNYTTA ANVDDNQRI IREL INNEAAVDALKRIATLSDQEKAMHSTLRANEKF DMDELEESPNYTTA $* * * * * * * * * * * * * * * * * * * * * * * * * * * * * * * * * * * * * * * * * * * * * * * * * * * * * * * * * * * *$

ENKS IRDYKDTQKALNDARMDFFTEKTKF IAKETLERGGQLYFALDGLVTNSPGFRADTQ ENKS IRDYKDTQKALNDARMDFFTEKTKF IAKETLERGGQLYFALDGLVTNSPGFRADTQ ENKSIRDYKDTQKALNDARMDFFTEKTKF IAKETLERGGQLYFALDGLVTNSPGFRADTQ ENKSIRDYKDTQKALNDARMDFFTEKTKFIAKETLERGGQLYFALDGLVTNSPGFRADTQ ENKS IRDYKDTQKALNDARMDFFTEKTKF IAKETLERGGQLYFALDGLVTNSPGFRADTQ ENKSIRDYKDTOKALNDARMDFFTEKTKF IAKETLERGGOLYFALDGLVTNSPGFRADTO ENKSIRDYKDTQKALNDARMDFFTEKTKFIAKETLERGGQLYFALDGLVTNSPGFRADTQ ENKSIRDYKDTQKALNDARMDFFTEKTKF IAKETLERGGQLYFALDGLVTNSPGFRADTQ ENKS IRDYKDTQKALNDARMDFFTEKTKF IAKETLERGGQLYFALDGLVTNSPGFRADTQ ENKSIRDYKDTQKALNDARMDFFTEKTKFIAKETLERGGQLYFALDGLVTNSPGFRADTQ 
NZS 1

NZS2

NZS3

NZS4

NZV1

$82-029362$

6644

HD183

HMC 46

HMC 56

$35000 \mathrm{HP}$

NZ 1

NZS2

NZS 3

NZS 4

NZV1

82-029362

6644

HD 183

HMC 46

HMC 56

$35000 \mathrm{HP}$

NZS1

NZS2

NZS 3

NZS4

NZV1

82-029362

6644

HD 183

HMC 46

HMC 56

$35000 \mathrm{HP}$

NZS 1

NZS2

NZS 3

NZS 4

NZV1

82-029362

6644

HD 183

HMC 46

HMC 56

$35000 \mathrm{HP}$

NZS 1

NZS2

NZS 3

NZS 4

NZV1

82-029362

6644

HD 183

HMC 46

HMC 56

$35000 \mathrm{HP}$

NZS 1

NZS2

NZS3

NZS4
INMDKLKDVFNPNHEHYDSVTSRELRYLYENYKDNPNLKFTLKDHVIANPLKTLKTSISE INMDKLKDVFNPNHEHYDSVTSRELRYLYENYKDNPNLKFTLKDHVIANPLKTLKTSISE INMDKLKDVFNPNHEHYDSVTSRELRYLYENYKDNPNLKFTLKDHVIANPLKTLKTSISE INMDKLKDVFNPNHEHYDSVTSRELRYLYENYKDNPNLKFTLKDHVIANPLKTLKTSISE INMDKLKDVFNPNHEHYDSVTSRELRYLYENYKDNPNLKFTLKDHVIANPLKTLKTSISE INMDKLKDVFNPNHEHYDSVTSRELRYLYENYKDNPNLKFTLKDHVIANPLKTLKTSISE INMDKLKDVFNPNHEHYDSVTSRELRYLYENYKDNPNLKFTLKDHVIANPLKTLKTSISE INMDKLKDVFNPNHEHYDSVTSRELRYLYENYKDNPNLKFTLKDHVIANPLKTLKTSISE INMDKLKDVFNPNHEHYDSVTSRELRYLYENYKDNPNLKFTLKDHVIANPLKTLKTSISE INMDKLKDVFNPNHEHYDSVTSRELRYLYENYKDNPNLKFTLKDHVIANPLKTLKTSISE INMDKLKDVFNPNHEHYDSVTSRELRYLYENYKDNPNLKFTLKDHVIANPLKTLKTSISE $* * * * * * * * * * * * * * * * * * * * * * * * * * * * * * * * * * * * * * * * * * * * * * * * * * * * * * * * * * * *$

SDLKSSPRRARQEGPSLLQRVRNLFDKSSSNKRSEKDTAQTSVGYRNTNIDI INDKTKGV SDLKSSPRRARQEGPSLLQRVRNLFDKSSSNKRSEKDTAQTSVGYRNTNIDI INDKTKGV SDLKSSPRRAROEGPSLLORVRNLFDKSSSNKRSEKDTAOTSVGYRNTNIDIINDKTKGV SDLKSSPRRARQEGPSLLQRVRNLFDKSSSNKRSEKDTAQTSVGYRNTNIDI INDKTKGV SDLKSSPRRAROEGPSLLQRVRNLFDKSSSNKRSEKDTAQTSVGYRNTNIDI INDKTKGV SDLKSSPRRARQEGPSLLQRVRNLFDKSSSNKRSEKDTAQTSVGYRNTNIDIINDKTKGV SDLKSSPRRARQEGPSLLQRVRNLFDKSSSNKRSEKDTAQTSVGYRNTNIDI INDKTKGV SDLKSSPRRARQEGPSLLQRVRNLFDKSSSNKRSEKDTAQTSVGYRNTNIDI INDKTKGV SDLKSSPRRARQEGPSLLQRVRNLFDKSSSNKRSEKDTAQTSVGYRNTNIDIINDKTKGV SDLKSSPRRARQEGPSLLQRVRNLFDKSSSNKRSEKDTAQTSVGYRNTNIDI INDKTKGV SDLKSSPRRARQEGPSLLQRVRNLFDKSSSNKRSEKDTAQTSVGYRNTNIDI INDKTKGV $\star * * * * * * * * * * * * * * * * * * * * * * * * * * * * * * * * * * * * * * * * * * * * * * * * * * * * * * * * * * * * * * * * * * *$

NHIVENGVEVALTNTDYKPLKLPNVEAAFKQTKLKAENIDPHIEAVKKLEI IASSANS IP NHIVENGVEVALTNTDYKPLKLPNVEAAFKQTKLKAENIDPH IEAVKKLEI IASSANS IP NHIVENGVEVALTNTDYKPLKLPNVEAAFKQTKLKAENIDPHIEAVKKLEI IASSANS IP NHIVENGVEVALTNTDYKPLKLPNVEAAFKQTKLKAENIDPHIEAVKKLEI IASSANS IP NHIVENGVEVALTNTDYKPLKLPNVEAAFKQTKLKAENIDPHIEAVKKLEIIASSANSIP NHIVENGVEVALTNTDYKPLKLPNVEAAFKQTKLKAENIDPHIEAVKKLEI IASSANS IP NH IVENGVEVALTNTDYKPLKLPNVEAAFKOTKLKAENIDPHIEAVKKLEI IASSANSIP NHIVENGVEVALTNTDYKPLKLPNVEAAFKQTKLKAENIDPHIEAVKKLEI IASSANS IP NHIVENGVEVALTNTDYKPLKLPNVEAAFKOTKLKAENIDPHIEAVKKLEI IASSANS IP NHIVENGVEVALTNTDYKPLKLPNVEAAFKQTKLKAENIDPHIEAVKKLEI IASSANS IP NHIVENGVEVALTNTDYKPLKLPNVEAAFKQTKLKAENIDPHIEAVKKLEIIASSANSIP $* * * * * * * * * * * * * * * * * * * * * * * * * * * * * * * * * * * * * * * * * * * * * * * * * * * * * * * * * * * * * * * *$

KEHLLKALIEVTEGKTDEDINVYOKLFNTRONISNEVAPTYSLRNLDGKDGKOILRSVAE KEHLLKALIEVTEGKTDEDINVYQKLFNTRQNISNEVAPTYSLRNLDGKDGKQILRSVAE KEHLLKALIEVTEGKTDEDINVYQKLFNTRQNISNEVAPTYSLRNLDGKDGKQILRSVAE KEHLLKALIEVTEGKTDEDINVYQKLFNTRQNISNEVAPTYSLRNLDGKDGKQILRSVAE KEHLLKALIEVTEGKTDEDINVYOKLFNTRONISNEVAPTYSLRNLDGKDGKOILRSVAE KEHLLKALIEVTEGKTDEDINVYQKLFNTRQNISNEVAPTYSLRNLDGKDGKQILRSVAE KEHLLKALIEVTEGKTDEDINVYQKLFNTRQNISNEVAPTYSLRNLDGKDGKQILRSVAE KEHLLKALIEVTEGKTDEDINVYQKLFNTRQNISNEVAPTYSLRNLDGKDGKQILRSVAE KEHLLKALIEVTEGKTDEDINVYQKLFNTRQNISNEVAPTYSLRNLDGKDGKOILRSVAE KEHLLKALIEVTEGKTDEDINVYQKLFNTRONISNEVAPTYSLRNLDGKDGKOILRSVAE KEHLLKALIEVTEGKTDEDINVYQKLFNTRQNISNEVAPTYSLRNLDGKDGKQILRSVAE $* * * * * * * * * * * * * * * * * * * * * * * * * * * * * * * * * * * * * * * * * * * * * * * * * * * * * * * * * * * * * * * * * * *$

IYKNLPLSDTYQAVRNYVNNRLIEKLSSNRLLLEHLANSKISGNEYAIKYIFDTVSRAKO IYKNLPLSDTYQAVRNYVNNRLIEKLSSNRLLLEHLANSK I SGNEYAIKY IFDTVSRAKQ IYKNLPLSDTYQAVRNYVNNRLIEKLSSNRLLLEHLANSKISGNEYAIKYIFDTVSRAKQ IYKNLPLSDTYQAVRNYVNNRLIEKLSSNRLLLEHLANSKISGNEYAIKYIFDTVSRAKQ IYKNLPLSDTYQAVRNYVNNRLIEKLSSNRLLLEHLANSKISGNEYAIKYIFDTVSRAKO IYKNLPLSDTYQAVRNYVNNRLIEKLSSNRLLLEHLANSKISGNEYAIKYIFDTVSRAKQ IYKNLPLSDTYQAVRNYVNNRLIEKLSSNRLLLEHLANSKISGNEYAIKYIFDTVSRAKQ IYKNLPLSDTYQAVRNYVNNRLIEKLSSNRLLLEHLANSKISGNEYAIKYIFDTVSRAKO IYKNLPLSDTYQAVRNYVNNRLIEKLSSNRLLLEHLANSK ISGNEYAIKY IFDTVSRAKQ IYKNLPLSDTYQAVRNYVNNRLIEKLSSNRLLLEHLANSKISGNEYAIKY IFDTVSRAKO IYKNLPLSDTYQAVRNYVNNRLIEKLS SNRLLLEHLANSKISGNEYAIKY IFDTVSRAKQ

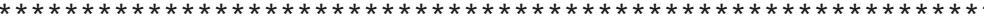

EIFEQELNTELAPVSLDIMRRKAANILSSEHGSYKDGTLS IYDKPVKSAFHSRLKNNGEV EIFEQELNTELAPVSLDIMRRKAANILSSEHGSYKDGTLS I YDKPVKSAFHSRLKNNGEV EIFEQELNTELAPVSLDIMRRKAANILSSEHGSYKDGTLS IYDKPVKSAFHSRLKNNGEV EIFEQELNTELAPVSLDIMRRKAANILSSEHGSYKDGTLS IYDKPVKSAFHSRLKNNGEV EIFEQELNTELAPVSLDIMRRKAANILSSEHGSYKDGTLS I YDKPVKSAFHSRLKNNGEV 
NZV1

82-029362

6644

HD 183

HMC 46

HMC 56

$35000 \mathrm{HP}$

NZS 1

NZS2

NZS3

NZS4

NZV1

82-029362

6644

HD 183

HMC 46

HMC 56

$35000 \mathrm{HP}$

NZS 1

NZS2

NZS 3

NZS 4

NZV1

82-029362

6644

HD 183

HMC 46

HMC 56

$35000 \mathrm{HP}$

NZS 1

NZS2

NZS3

NZS4

NZV1

82-029362

6644

HD 183

HMC 46

HMC 56

EIFEQELNTELAPVSLDIMRRKAANILSSEHGSYKDGTLS IYDKPVKSAFHSRLKNNGEV EIFEOELNTELAPVSLDIMRRKAANILSSEHGSYKDGTLS IYDKPVKSAFHSRLKNNGEV EIFEQELNTELAPVSLDIMRRKAANILSSEHGSYKDGTLS IYDKPVKSAF HSRLKNNGEV EIFEOELNTELAPVSLDIMRRKAAN ILSSEHGSYKDGTLS IYDKPVKSAFHSRLKNNGEV EIFEQELNTELAPVSLDIMRRKAANILSSEHGSYKDGTLS IYDKPVKSAFHSRLKNNGEV EIFEQELNTELAPVSLDIMRRKAAN ILSSEHGSYKDGTLS IYDKPVKSAFHSRLKNNGEV $* * * * * * * * * * * * * * * * * * * * * * * * * * * * * * * * * * * * * * * * * * * * * * * * * * * * * * * * * * * *$

LNTIVHELTHHEODALAKI IDNKGYDAKLFDKNNILYITGGLGYPKOALERDAFLSGDSV LNTIVHELTHHEQDALAKI IDNKGYDAKLFDKNN ILYITGGLGYPKQALERDAFLSGDSV LNTIVHELTHHEODALAKI IDNKGYDAKLFDKNN ILY ITGGLGYPKOALERDAFLSGDSV LNTIVHELTHHEQDALAKI IDNKGYDAKLFDKNNILYITGGLGYPKQALERDAFLSGDSV LNTIVHELTHHEQDALAKI IDNKGYDAKLFDKNN ILYITGGLGYPKQALERDAFLSGDSV LNTIVHELTHHEQDALAKI IDNKGYDAKLFDKNN ILYITGGLGYPKQALERDAFLSGDSV LNT IVHELTHHEQDALAKI IDNKGYDAKLFDKNN ILYITGGLGYPKQALERDAFLSGDSV LNTIVHELTHHEODALAKI IDNKGYDAKLFDKNN ILYITGGLGYPKOALERDAFLSGDSV LNTIVHELTHHEQDALAKI IDNKGYDAKLFDKNNILYITGGLGYPKQALERDAFLSGDSV LNTIVHELTHHEQDALAKI IDNKGYDAKLFDKNN ILYITGGLGYPKQALERDAFLSGDSV LNTIVHELTHHEQDALAKI IDNKGYDAKLFDKNNILYITGGLGYPKQALERDAFLSGDSV $* * * * * * * * * * * * * * * * * * * * * * * * * * * * * * * * * * * * * * * * * * * * * * * * * * * * * * * * * * * *$

SEAFMKKAKEYHERTKQERKDAKKDEARIAKLYKQWEQEEANKKSASLNGSSQSLDSRSE SEAFMKKAKEYHERTKQERKDAKKDEARIAKLYKQWEQEEANKKSASLNGSSQSLDSRSE SEAFMKKAKEYHERTKQERKDAKKDEARIAKLYKQWEQEEANKKSASLNGSSQSLDSRSE SEAFMKKAKEYHERTKQERKDAKKDEARIAKLYKQWEQEEANKKSASLNGSSQSLDSRSE SEAFMKKAKEYHERTKQERKDAKKDEARIAKLYKQWEQEEANKKSASLNGSSQSLDSRSE SEAFMKKAKEYHERTKQERKDAKKDEARIAKLYKQWEQEEANKKSASLNGSSQSLDSRSE SEAFMKKAKEYHERTKOERKDAKKDEARIAKLYKOWEOEEANKKSASLNGSSOSLDSRSE SEAFMKKAKEYHERTKQERKDAKKDEARIAKLYKQWEQEEANKKSASLNGSSQSLDSRSE SEAFMKKAKEYHERTKQERKDAKKDEARIAKLYKQWEQEEANKKSASLNGSSQSLDSRSE SEAFMKKAKEYHERTKQERKDAKKDEARIAKLYKQWEQEEANKKSASLNGSSQSLDSRSE SEAFMKKAKEYHERTKQERKDAKKDEARIAKLYKQWEQEEANKKSASLNGSSQSLDSRSE $* * * * * * * * * * * * * * * * * * * * * * * * * * * * * * * * * * * * * * * * * * * * * * * * * * * * * * * * * * * *$

VEFNRVSHKSVR* VEFNRVSHKSVR * VEFNRVSHKSVR * VEFNRVSHKSVR * VEFNRVSHKSVR * VEFNRVSHKSVR * VEFNRVSHKSVR* VEFNRVSHKSVR * VEFNRVSHKSVR * VEFNRVSHKSVR * VEFNRVSHKSVR*

$* * * * * * * * * * * * *$

Note: Due to difficulty in sorting reads of 1 spAl and 1 spA2 genes of class II strains, LspAl sequences of class II strains were excluded from this analysis.

\section{3) LspA2 (HD1156)}

$35000 \mathrm{HP}$

NZS 1

NZS2

NZS3

NZS4

NZV1

82-029362

6644

HD 183

HMC 46

HMC 56

$35000 \mathrm{HP}$

NZS 1

NZS2

NZS 3

NZS 4

NZV1

82-029362
MNNKRYKLIFSKVKNCLVPVAENIKSASGNSGSSSNSKIAEDOEEEPDSLACSLSPLSSS MNNKRYKLIFSKVKNCLVPVAENIKSASGNSGSSSNSKIAEDQEEEPDSLACSLSPLSSS MNNKRYKLIFSKVKNCLVPVAENIKSASGNSGSSSNSKIAEDQEEEPDSLACSLSPLSSS MNNKRYKLIFSKVKNCLVPVAENIKSASGNSGSSSNSKIAEDQEEEPDSLACSLSPLSSS MNNKRYKLIFSKVKNCLVPVAENIKSASGNSGSSSNSKIAEDQEEEPDSLACSLSPLSSS MNNKRYKLIFSKVKNCLVPVAENIKSASGNSGSSSNSKIAEDOEEEPDSLACSLSPLSSS MNNKRYKLIFSKVKNCLVPVAENIKSASGNSGSSSNSKIAEDQEEEPDSLACSLSPLSSS MNNKRYKLIFSKVKNCLVPVAENIKSASGNSGSSSNSKIAEDQEEEPDSLACSLSPLSSS MNNKRYKLIFSKVKNCLVPVAENIKSASGNSGSSSNSKIAEDOEEEPDSLACSLSPLSSS MNNKRYKLIFSKVKNCLVPVAENIKSASGNSGSSSNSKIAEDQEEEPDSLACSLSPLSSS MNNKRYKLIFSKVKNCLVPVAENIKSASGNSGSSSNSKIAEDQEEEPDSLACSLSPLSSS $* * * * * * * * * * * * * * * * * * * * * * * * * * * * * * * * * * * * * * * * * * * * * * * * * * * * * * * * * * * *$

IHLGLHNHSPLKVFKGKNLSVVLLSLMPAMQVWADSSNAIVDHSHGAKQTAVDERDPKNG IHLGLHNHSPLKVFKGKNLSVVLLSLMPAMQVWADSSNAIVDHSHGAKQTAVDERDPKNG IHLGLHNHSPLKVFKGKNLSVVLLSLMPAMQVWADSSNAIVDHSHGAKQTAVDERDPKNG IHLGLHNHSPLKVFKGKNLSVVLLSLMPAMQVWADSSNAIVDHSHGAKQTAVDERDPKNG IHLGLHNHSPLKVFKGKNLSVVLLSLMPAMOVWADSSNAIVDHSHGAKQTAVDERDPKNG IHLGLHNHSPLKVFKGKNLSVVLLSLMPAMQVWADSSNAIVDHSHGAKQTAVDERDPKNG IHLGLHNHSPLKVFKGKNLSVVLLSLMPAMQVWADSSNAIVDHSHGAKQTAVDERDPKNG 
6644

HD 183

HMC 46

HMC 56

$35000 \mathrm{HP}$

NZS 1

NZS2

NZS 3

NZS 4

NZV1

82-029362

6644

HD 183

HMC 46

HMC 56

$35000 \mathrm{HP}$

NZS 1

NZS2

NZS 3

NZS 4

NZV1

82-029362

6644

HD 183

HMC 46

HMC 56

$35000 \mathrm{HP}$

NZS 1

NZS2

NZS 3

NZS 4

NZV1

82-029362

6644

HD 183

HMC 46

HMC 56

$35000 \mathrm{HP}$

NZ S 1

NZS2

NZS 3

NZS 4

NZV1

82-029362

6644

HD 183

HMC 46

HMC 56

$35000 \mathrm{HP}$

NZS 1

NZS2

NZS 3

NZS4

NZV1

82-029362

6644

HD 183

HMC 46

HMC 56
IHLGLHNHSPLKVFKGKNLSVVLLSLMPAMOVWADSSNAIVDHSHGAKQTAVDERDPKNG IHLGLHNHSPLKVFKGKNLSVVLLSLMPAMQVWADSSNAIVDHSHGAKOTAVDERDPKNG IHLGLHNHSPLKVFKGKNLSVVLLSLMPAMQVWADSSNAIVDHSHGAKQTAVDERDPKNG IHLGLHNHSPLKVFKGKNLSVVLLSLMPAMOVWADSSNAIVDHSHGAKOTAVDERDPKNG $* * * * * * * * * * * * * * * * * * * * * * * * * * * * * * * * * * * * * * * * * * * * * * * * * * * * * * * * * * * *$

KEKVVVINIAKPDEQGISDNHFSKFNIPNSAVFNNSIKEGNSQLVGLLGENKNLGSQAAK KEKVVVINIAKPDEQGISDNHF SKFNIPNSAVFNNSIKEGNSQLVGLLGENKNLGSQAAK KEKVVVINIAKPDEOGISDNHFSKFNIPNSAVFNNSIKEGNSOLVGLLGENKNLGSOAAK KEKVVVINIAKPDEQGISDNHF SKFNIPNSAVFNNS I KEGNSQLVGLLGENKNLGSQAAK KEKVVVINIAKPDEOGISDNHF SKFNIPNSAVFNNSIKEGNSOLVGLLGENKNLGSOAAK KEKVVVINIAKPDEQGISDNHF SKFNI PNSAVFNNSIKEGNSQLVGLLGENKNLGSQAAK KEKVVVINIAKPDEQGISDNHF SKFNI PNSAVFNNSIKEGNSQLVGLLGENKNLGSQAAK KEKVVVINIAKPDEOGISDNHF SKFNI PNSAVFNNS I KEGNSOLVGLLGENKNLGSOAAK KEKVVVINIAKPDEQGISDNHF SKFNIPNSAVFNNSIKEGNSQLVGLLGENKNLGSQAAK KEKVVVINIAKPDEQGISDNHF SKFNI PNSAVFNNS I KEGNSQLVGLLGENKNLGSQAAK KEKVVVINIAKPDEQGISDNHFSKFNI PNSAVFNNS I KEGNSQLVGLLGENKNLGSQAAK $\star * * * * * * * * * * * * * * * * * * * * * * * * * * * * * * * * * * * * * * * * * * * * * * * * * * * * * * * * * * *$

TIFNQVTGDQESKISGGLEVFGEKADLF I INPNGVTLNGVKTINTDRFVASTSEVVEPH I TIFNQVTGDQESK ISGGLEVFGEKADLF I INPNGVTLNGVKTINTDRFVAST SEVVEPH I TIFNQVTGDQESKISGGLEVFGEKADLF I INPNGVTLNGVKTINTDRFVASTSEVVEPH I TIFNOVTGDOESKISGGLEVFGEKADLF I INPNGVTLNGVKTINTDRFVASTSEVVEPH I TIFNQVTGDQESK ISGGLEVFGEKADLF I INPNGVTLNGVKTINTDRFVASTSEVVEPH I TIFNQVTGDQESKISGGLEVFGEKADLF I INPNGVTLNGVKTINTDRFVASTSEVVEPH I TIFNQVTGDQESKISGGLEVFGEKADLF I INPNGVTLNGVKTINTDRFVASTSEVVEPH I TIFNQVTGDQESK I SGGLEVFGEKADLF I INPNGVTLNGVKTINTDRFVASTSEVVEPHI TIFNOVTGDOESK ISGGLEVFGEKADLF I INPNGVTLNGVKTINTDRFVASTSEVVEPH I TIFNQVTGDQESKISGGLEVFGEKADLF I INPNGVTLNGVKTINTDRFVASTSEVVEPH I TIFNOVTGDQESK ISGGLEVFGEKADLF I INPNGVTLNGVKT INTDRFVASTSEVVEPH I $* * * * * * * * * * * * * * * * * * * * * * * * * * * * * * * * * * * * * * * * * * * * * * * * * * * * * * * * * * * * * * * * *$

KOLNVORGKVIIGKDGVATNGLSHFDVVAKNIEOOGKVSIEGDSKPAKLANVTFAAGNLT KQLNVQRGKVIIGKDGVATNGLSHFDVVAKNIEQQGKVSIEGDSKPAKLANVTFAAGNLT KQLNVQRGKVIIGKDGVATNGLSHFDVVAKNIEQQGKVSIEGDSKPAKLANVTFAAGNLT KQLNVQRGKVIIGKDGVATNGLSHFDVVAKNIEQQGKVSIEGDSKPAKLANVTFAAGNLT KQLNVQRGKVIIGKDGVATNGLSHFDVVAKNIEQQGKVSIEGDSKPAKLANVTFAAGNLT KOLNVORGKVIIGKDGVATNGLSHFDVVAKNIEOOGKVSIEGDSKPAKLANVTFAAGNLT KQLNVQRGKVIIGKDGVATNGLSHFDVVAKNIEQQGKVSIEGDSKPAKLANVTFAAGNLT KOLNVORGKVIIGKDGVATNGLSHFDVVAKNIEOOGKVSIEGDSKPAKLANVTFAAGNLT KQLNVQRGKVIIGKDGVATNGLSHFDVVAKNIEQQGKVS IEGDSKPAKLANVTFAAGNLT KQLNVQRGKVI IGKDGVATNGLSHFDVVAKNIEQQGKVSIEGDSKPAKLANVTFAAGNLT KOLNVQRGKVIIGKDGVATNGLSHFDVVAKNIEOOGKVS IEGDSKPAKLANVTFAAGNLT $* * * * * * * * * * * * * * * * * * * * * * * * * * * * * * * * * * * * * * * * * * * * * * * * * * * * * * * * * * * *$

YDVNTRDVNRNTNPKKPITDNTRKDNIAISGESAGSMYGRNIKF IVTDKGAGVNHOGVIF YDVNTRDVNRNTNPKKPITDNTRKDNIAISGESAGSMYGRNIKF IVTDKGAGVNHQGVIF YDVNTRDVNRNTNPKKPITDNTRKDNIAISGESAGSMYGRNIKF IVTDKGAGVNHOGVIF YDVNTRDVNRNTNPKKPITDNTRKDNIAISGESAGSMYGRNIKF IVTDKGAGVNHOGVIF YDVNTRDVNRNTNPKKPITDNTRKDNIAISGESAGSMYGRNIKF IVTDKGAGVNHOGVIF YDVNTRDVNRNTNPKKPITDNTRKDNIAISGESAGSMYGRNI KF IVTDKGAGVNHQGVIF YDVNTRDVNRNTNPKKPITDNTRKDNIAISGESAGSMYGRNIKF IVTDKGAGVNHOGVIF YDVNTRDVNRNTNPKKPITDNTRKDNIAISGESAGSMYGRNIKF IVTDKGAGVNHOGVIF YDVNTRDVNRNTNPKKPITDNTRKDNIAISGESAGSMYGRNIKF IVTDKGAGVNHOGVIF YDVNTRDVNRNTNPKKPITDNTRKDNIAISGESAGSMYGRNIKF IVTDKGAGVNHOGVIF YDVNTRDVNRNTNPKKPITDNTRKDNIAISGESAGSMYGRNIKF IVTDKGAGVNHQGVIF $* * * * * * * * * * * * * * * * * * * * * * * * * * * * * * * * * * * * * * * * * * * * * * * * * * * * * * * * * * * * *$

AEDDINILTDDGNSRLNKVYADYVRVVGKDIELANNGQIHADQQLILNATGHVKLNDGSS AEDDINILTDDGNSRLNKVYADYVRVVGKDIELANNGQIHADQQLILNATGHVKLNDGSS AEDDINILTDDGNSRLNKVYADYVRVVGKDIELANNGQIHADQQLILNATGHVKLNDGSS AEDDINILTDDGNSRLNKVYADYVRVVGKDIELANNGQIHADQQLILNATGHVKLNDGSS AEDDINILTDDGNSRLNKVYADYVRVVGKDIELANNGQIHADQQLILNATGHVKLNDGSS AEDDINILTDDGNSRLNKVYADYVRVVGKDIELANNGOIHADOQLILNATGHVKLNDGSS AEDDINILTDDGNSRLNKVYADYVRVVGKDIELANNGQIHADQQLILNATGHVKLNDGSS AEDDINILTDDGNSRLNKVYADYVRVVGKDIELANNGQIHADQQLILNATGHVKLNDGSS AEDDINILTDDGNSRLNKVYADYVRVVGKDIELANNGQIHADQQLILNATGHVKLNDGSS AEDDINILTDDGNSRLNKVYADYVRVVGKDIELANNGQIHADQQLILNATGHVKLNDGSS AEDDINILTDDGNSRLNKVYADYVRVVGKDIELANNGQIHADQQLILNATGHVKLNDGSS $* * * * * * * * * * * * * * * * * * * * * * * * * * * * * * * * * * * * * * * * * * * * * * * * * * * * * * * * * * * *$

VISNNNLGISALNLTLENATVSANNLSFRVTNDTKLNNLSKVSARAADLOSGNLNLDKAS VISNNNLGISALNLTLENATVSANNLSFRVTNDTKLNNLSKVSARAADLQSGNLNLDKAS 
NZS2

NZS 3

NZS4

NZV1

82-029362

6644

HD 183

HMC 46

HMC 56

$35000 \mathrm{HP}$

NZS 1

NZS2

NZS 3

NZS 4

NZV1

82-029362

6644

HD 183

HMC 46

HMC 56

$35000 \mathrm{HP}$

NZS 1

NZS2

NZS 3

NZS 4

NZV1

82-029362

6644

HD 183

HMC 46

HMC 56

$35000 \mathrm{HP}$

NZS 1

NZS2

NZS 3

NZS4

NZV1

82-029362

6644

HD 183

HMC 46

HMC 56

$35000 \mathrm{HP}$

NZS 1

NZS2

NZS 3

NZS 4

NZV1

82-029362

6644

HD 183

HMC 46

HMC 56

$35000 \mathrm{HP}$

NZS1

NZS2

NZS3

NZS 4

NZV1

$82-029362$

6644

HD 183

HMC 46
VISNNNLGISALNLTLENATVSANNLSFRVTNDTKLNNLSKVSARAADLQSGNLNLDKAS VISNNNLGISALNLTLENATVSANNLSFRVTNDTKLNNLSKVSARAADLQSGNLNLDKAS VISNNNLGISALNLTLENATVSANNLSFRVTNDTKLNNLSKVSARAADLQSGNLNLDKAS VISNNNLGISALNLTLENATVSANNLSFRVTNDTKLNNLSKVSARAADLQSGNLNLDKAS VISNNNLGISALNLTLENATVSANNLSFRVTNDTKLNNLSKVSARAADLQSGNLNLDKAS VISNNNLGISALNLTLENATVSANNLSFRVTNDTKLNNLSKVSARAADLQSGNLNLDKAS VISNNNLGISALNLTLENATVSANNLSFRVTNDTKLNNLSKVSARAADLQSGNLNLDKAS VISNNNLGISALNLTLENATVSANNLSFRVTNDTKLNNLSKVSARAADLQSGNLNLDKAS VISNNNLGISALNLTLENATVSANNLSFRVTNDTKLNNLSKVSARAADLQSGNLNLDKAS $* * * * * * * * * * * * * * * * * * * * * * * * * * * * * * * * * * * * * * * * * * * * * * * * * * * * * * * * * * * *$

VLAHKLTLNISNDVSLNNQSKLSANNLKIKKVRDLNLNNSELSANNLTLNTSNNITLKNK VLAHKLTLNISNDVSLNNQSKLSANNLKIKKVRDLNLNNSELSANNLTLNTSNNITLKNK VLAHKLTLNISNDVSLNNQSKLSANNLKIKKVRDLNLNNSELSANNLTLNTSNNITLKNK VLAHKLTLNISNDVSLNNQSKLSANNLKIKKVRDLNLNNSELSANNLTLNTSNNITLKNK VLAHKLTLNISNDVSLNNQSKLSANNLKI KKVRDLNLNNSELSANNLTLNTSNNITLKNK VLAHKLTLNISNDVSLNNQSKLSANNLKIKKVRDLNLNNSELSANNLTLNTSNNITLKNK VLAHKLTLNISNDVSLNNQSKLSANNLKIKKVRDLNLNNSELSANNLTLNTSNNITLKNK VLAHKLTLNISNDVSLNNQSKLSANNLKIKKVRDLNLNNSELSANNLTLNTSNNITLKNK VLAHKLTLNISNDVSLNNQSKLSANNLKI KKVRDLNLNNSELSANNLTLNTSNNITLKNK VLAHKLTLNISNDVSLNNOSKLSANNLKI KKVRDLNLNNSELSANNLTLNTSNNITLKNK VLAHKLTLNISNDVSLNNQSKLSANNLKI KKVRDLNLNNSELSANNLTLNTSNNITLKNK $* * * * * * * * * * * * * * * * * * * * * * * * * * * * * * * * * * * * * * * * * * * * * * * * * * * * * * * * * * * *$

SKFTAGNMTLNVTNNVTLNNDSELAANNLTLNVTKNVTLNDASKLSANKLDLNVTDNVTL SKFTAGNMTLNVTNNVTLNNDSELAANNLTLNVTKNVTLNDASKLSANKLDLNVTDNVTL SKFTAGNMTLNVTNNVTLNNDSELAANNLTLNVTKNVTLNDASKLSANKLDLNVTDNVTL SKFTAGNMTLNVTNNVTLNNDSELAANNLTLNVTKNVTLNDASKLSANKLDLNVTDNVTL SKFTAGNMTLNVTNNVTLNNDSELAANNLTLNVTKNVTLNDASKLSANKLDLNVTDNVTL SKFTAGNMTLNVTNNVTLNNDSELAANNLTLNVTKNVTLNDASKLSANKLDLNVTDNVTL SKFTAGNMTLNVTNNVTLNNDSELAANNLTLNVTKNVTLNDASKLSANKLDLNVTDNVTL SKFTAGNMTLNVTNNVTLNNDSELAANNLTLNVTKNVTLNDASKLSANKLDLNVTDNVTL SKFTAGNMTLNVTNNVTLNNDSELAANNLTLNVTKNVTLNDASKLSANKLDLNVTDNVTL SKFTAGNMTLNVTNNVTLNNDSELAANNLTLNVTKNVTLNDASKLSANKLDLNVTDNVTL SKFTAGNMTLNVTNNVTLNNDSELAANNLTLNVTKNVTLNDASKLSANKLDLNVTDNVTL $* * * * * * * * * * * * * * * * * * * * * * * * * * * * * * * * * * * * * * * * * * * * * * * * * * * * * * * * * * * *$

NSKSTLSAGELTFKKVKNVTLNNDSELAANNLSLNASHNVTLNNKSKLSAQKADIKAVNL NSKSTLSAGELTFKKVKNVTLNNDSELAANNLSLNASHNVTLNNKSKLSAQKADIKAVNL NSKSTLSAGELTFKKVKNVTLNNDSELAANNLSLNASHNVTLNNKSKLSAOKADIKAVNL NSKSTLSAGELTFKKVKNVTLNNDSELAANNLSLNASHNVTLNNKSKLSAQKADIKAVNL NSKSTLSAGELTFKKVKNVTLNNDSELAANNLSLNASHNVTLNNKSKLSAQKADIKAVNL NSKSTLSAGELTFKKVKNVTLNNDSELAANNLSLNASHNVTLNNKSKLSAOKADIKAVNL NSKSTLSAGELTFKKVKNVTLNNDSELAANNLSLNASHNVTLNNKSKLSAQKADIKAVNL NSKSTLSAGELTFKKVKNVTLNNDSELAANNLSLNASHNVTLNNKSKLSAOKADIKAVNL NSKSTLSAGELTFKKVKNVTLNNDSELAANNLSLNASHNVTLNNKSKLSAQKADIKAVNL NSKSTLSAGELTFKKVKNVTLNNDSELAANNLSLNASHNVTLNNKSKLSAOKADIKAVNL NSKSTLSAGELTFKKVKNVTLNNDSELAANNLSLNASHNVTLNNKSKLSAQKADIKAVNL $* * * * * * * * * * * * * * * * * * * * * * * * * * * * * * * * * * * * * * * * * * * * * * * * * * * * * * * * * * * *$

TLNDTTELTAKNLDINSTTITNNGTIAGIFANITTEKLNNKEKALILAEQNLNFTVNGSH TLNDTTELTAKNLDINSTTITNNGTIAGIFANITTEKLNNKEKALILAEQNLNFTVNGSH TLNDTTELTAKNLDINSTTITNNGTIAGIFANITTEKLNNKEKALILAEQNLNFTVNGSH TLNDTTELTAKNLDINSTTITNNGTIAGIFANITTEKLNNKEKALILAEQNLNFTVNGSH TLNDTTELTAKNLDINSTTITNNGTIAGIFANITTEKLNNKEKALILAEQNLNFTVNGSH TLNDTTELTAKNLDINSTTITNNGTIAGIFANITTEKLNNKEKALILAEQNLNFTVNGSH TLNDTTELTAKNLDINSTTITNNGTIAGIFANITTEKLNNKEKALILAEONLNFTVNGSH TLNDTTELTAKNLDINSTTITNNGTIAGIFANITTEKLNNKEKALILAEQNLNFTVNGSH TLNDTTELTAKNLDINSTTITNNGTIAGIFANITTEKLNNKEKALILAEQNLNFTVNGSH TLNDTTELTAKNLDINSTTITNNGTIAGIFANITTEKLNNKEKALILAEONLNFTVNGSH TLNDTTELTAKNLDINSTTITNNGTIAGIFANITTEKLNNKEKALILAEQNLNFTVNGSH $* * * * * * * * * * * * * * * * * * * * * * * * * * * * * * * * * * * * * * * * * * * * * * * * * * * * * * * * * * * *$

YENKGDIVSKDKATVTFSKNSDFTSNGSKLVNAONQLKVNVNNFTISOGDDITLIGNVTL YENKGDIVSKDKATVTFSKNSDFTSNGSKLVNAQNQLKVNVNNFTISQGDDITLIGNVTL YENKGDIVSKDKATVTFSKNSDFTSNGSKLVNAQNQLKVNVNNFTISQGDDITLIGNVTL YENKGDIVSKDKATVTFSKNSDFTSNGSKLVNAQNQLKVNVNNFTISQGDDITLIGNVTL YENKGDIVSKDKATVTFSKNSDFTSNGSKLVNAQNQLKVNVNNFTISQGDDITLIGNVTL YENKGDIVSKDKATVTFSKNSDFTSNGSKLVNAONOLKVNVNNFTISOGDDITLIGNVTL YENKGDIVSKDKATVTFSKNSDFTSNGSKLVNAQNQLKVNVNNFTISQGDDITLIGNVTL YENKGDIVSKDKATVTFSKNSDFTSNGSKLVNAQNQLKVNVNNFTISOGDDITLIGNVTL YENKGDIVSKDKATVTFSKNSDFTSNGSKLVNAONOLKVNVNNFTISOGDDITLIGNVTL YENKGDIVSKDKATVTFSKNSDFTSNGSKLVNAQNQLKVNVNNFTISQGDDITLIGNVTL 
NZS 1

NZS2

NZS3

NZS4

NZV1

$82-029362$

6644

HD183

HMC 46

HMC 56

$35000 \mathrm{HP}$

NZ 1

NZS2

NZS 3

NZS 4

NZV1

82-029362

6644

HD 183

HMC 46

HMC 56

$35000 \mathrm{HP}$

NZS1

NZS2

NZS 3

NZS4

NZV1

82-029362

6644

HD 183

HMC 46

HMC 56

$35000 \mathrm{HP}$

NZS1

NZS2

NZS 3

NZS 4

NZV1

82-029362

6644

HD 183

HMC 46

HMC 56

$35000 \mathrm{HP}$

NZS 1

NZS2

NZS 3

NZS 4

NZV1

82-029362

6644

HD 183

HMC 46

HMC 56

$35000 \mathrm{HP}$

NZS 1

NZS2

NZS3

NZS4
NASGTFTNSGNLTTVKTLDVGDIONFTNKGNLTVGEDLHIKSKTKITNDGKLIS IKNLN I NASGTFTNSGNLTTVKTLDVGDIQNFTNKGNLTVGEDLHIKSKTKITNDGKLISIKNLNI NASGTFTNSGNLTTVKTLDVGDIQNFTNKGNLTVGEDLHIKSKTKITNDGKLIS IKNLN I NASGTFTNSGNLTTVKTLDVGDIONFTNKGNLTVGEDLHIKSKTKITNDGKLISIKNLNI NASGTFTNSGNLTTVKTLDVGDIQNFTNKGNLTVGEDLHIKSKTKITNDGKLISIKNLNI NASGTFTNSGNLTTVKTLDVGDIQNFTNKGNLTVGEDLHIKSKTKITNDGKLISIKNLNI NASGTFTNSGNLTTVKTLDVGDIQNFTNKGNLTVGEDLHIKSKTKITNDGKLIS IKNLN I NASGTFTNSGNLTTVKTLDVGDIONFTNKGNLTVGEDLHIKSKTKITNDGKLISIKNLNI NASGTFTNSGNLTTVKTLDVGDIQNFTNKGNLTVGEDLHIKSKTKITNDGKLISIKNLN I NASGTFTNSGNLTTVKTLDVGDIQNFTNKGNLTVGEDLHIKSKTKITNDGKLIS IKNLNI NASGTFTNSGNLTTVKTLDVGDIONFTNKGNLTVGEDLHIKSKTKITNDGKLIS IKNLN I $* * * * * * * * * * * * * * * * * * * * * * * * * * * * * * * * * * * * * * * * * * * * * * * * * * * * * * * * * * * *$

SSEADF INNGTLLGIEALKIATKGNFTNKEKAILASNSLLDISVAEGKKTFNNGTIESGK SSEADF INNGTLLGIEALKIATKGNFTNKEKAILASNSLLDISVAEGKKTFNNGTIESGK SSEADF INNGTLLGIEALKIATKGNFTNKEKAILASNSLLDISVAEGKKTFNNGTIESGK SSEADF INNGTLLGIEALKIATKGNFTNKEKAILASNSLLDISVAEGKKTFNNGTIESGK SSEADF INNGTLLGIEALKIATKGNFTNKEKAILASNSLLDISVAEGKKTFNNGTIESGK SSEADF INNGTLLGIEALKIATKGNFTNKEKAILASNSLLDISVAEGKKTFNNGTIESGK SSEADF INNGTLLGIEALKIATKGNFTNKEKAILASNSLLDISVAEGKKTFNNGTIESGK SSEADF INNGTLLGIEALK IATKGNFTNKEKAILASNSLLDISVAEGKKTFNNGTIESGK SSEADF INNGTLLGIEALKIATKGNFTNKEKAILASNSLLDISVAEGKKTFNNGTIESGK SSEADF INNGTLLGIEALKIATKGNFTNKEKAILASNSLLDISVAEGKKTFNNGTIESGK SSEADF INNGTLLGIEALKIATKGNFTNKEKAILASNSLLDISVAEGKKTFNNGTIESGK

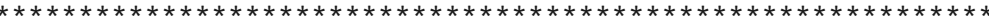

NLNITNTGAFLNVDNATIRSFGVLNITSTGNVSNNGTLISNERLNITSAANFTNESNGTV NLNITNTGAFLNVDNATIRSFGVLNITSTGNVSNNGTLISNERLNITSAANFTNESNGTV NLNITNTGAFLNVDNATIRSFGVLNITSTGNVSNNGTLISNERLNITSAANFTNESNGTV NLNITNTGAFLNVDNATIRSFGVLNITSTGNVSNNGTLISNERLNITSAANFTNESNGTV NLNITNTGAFLNVDNATIRSFGVLNITSTGNVSNNGTLISNERLNITSAANFTNESNGTV NLNITNTGAFLNVDNATIRSFGVLNITSTGNVSNNGTLISNERLNITSAANFTNESNGTV NLNITNTGAFLNVDNATIRSFGVLNITSTGNVSNNGTLISNERLNITSAANFTNESNGTV NLNITNTGAFLNVDNATIRSFGVLNITSTGNVSNNGTLISNERLNITSAANFTNESNGTV NLNITNTGAFLNVDNATIRSFGVLNITSTGNVSNNGTLISNERLNITSAANFTNESNGTV NLNITNTGAFLNVDNATIRSFGVLNITSTGNVSNNGTLISNERLNITSAANFTNESNGTV NLNITNTGAFLNVDNATIRSFGVLNITSTGNVSNNGTLISNERLNITSAANFTNESNGTV $* * * * * * * * * * * * * * * * * * * * * * * * * * * * * * * * * * * * * * * * * * * * * * * * * * * * * * * * * * * * * * *$

MSNGLLNIIAKOGNITNKNLIASRQOLNLTAVADNITNDSNISNKIAVLHSLGNISLNSK MSNGLLNI IAKQGNITNKNLIASRQQLNLTAVADNITNDSNISNKIAVLHSLGNISLNSK MSNGLLNI IAKOGNITNKNLIASROOLNLTAVADNITNDSNISNK IAVLHSLGNISLNSK MSNGLLNI IAKQGNITNKNLIASRQQLNLTAVADNITNDSNISNKIAVLHSLGNISLNSK MSNGLLNI IAKQGNITNKNLIASRQQLNLTAVADNITNDSNISNKIAVLHSLGNISLNSK MSNGLLNI IAKQGNITNKNLIASRQQLNLTAVADNITNDSNISNKIAVLHSLGNISLNSK MSNGLLNI IAKQGNITNKNLIASRQQLNLTAVADNITNDSNISNKIAVLHSLGNISLNSK MSNGLLNI IAKQGNITNKNLIASRQOLNLTAVADNITNDSNISNKIAVLHSLGNISLNSK MSNGLLNI IAKOGNITNKNLIASRQQLNLTAVADNITNDSNISNKIAVLHSLGNISLNSK MSNGLLNI IAKQGNITNKNLIASRQQLNLTAVADNITNDSNISNKIAVLHSLGNISLNSK MSNGLLNI IAKOGNITNKNLIASRQQLNLTAVADNITNDSNISNKIAVLHSLGNISLNSK $* * * * * * * * * * * * * * * * * * * * * * * * * * * * * * * * * * * * * * * * * * * * * * * * * * * * * * * * * * * * * * * * *$

DQVYNLGEIYAGNNISVKAHQLKNDVKLMGDITTKTKEGQASYKLYQASNGGHFGNDGSS DQVYNLGEIYAGNNISVKAHQLKNDVKLMGDITTKTKEGQASYKLYQASNGGHFGNDGSS DQVYNLGEIYAGNNISVKAHQLKNDVKLMGDITTKTKEGQASYKLYQASNGGHFGNDGSS DQVYNLGEIYAGNNISVKAHQLKNDVKLMGDITTKTKEGQASYKLYQASNGGHFGNDGSS DQVYNLGEIYAGNNISVKAHOLKNDVKLMGDITTKTKEGQASYKLYQASNGGHFGNDGSS DQVYNLGEIYAGNNISVKAHQLKNDVKLMGDITTKTKEGQASYKLYQASNGGHF GNDGSS DQVYNLGE I YAGNNISVKAHQLKNDVKLMGDITTKTKEGQASYKLYQASNGGHF GNDGSS DQVYNLGEIYAGNNISVKAHQLKNDVKLMGDITTKTKEGQASYKLYQASNGGHFGNDGSS DOVYNLGEIYAGNNISVKAHOLKNDVKLMGDITTKTKEGQASYKLYQASNGGHFGNDGSS DQVYNLGEIYAGNNISVKAHQLKNDVKLMGDITTKTKEGQASYKLYQASNGGHFGNDGSS DQVYNLGEIYAGNNISVKAHQLKNDVKLMGDITTKTKEGQASYKLYQASNGGHFGNDGSS

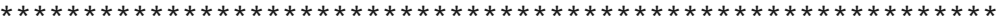

GYSEGDLNIKGKFADLDNKLTVORIGKIYAGRDLTFNKSNAGGKSEI INRGTINVKNKLS GYSEGDLNIKGKF ADLDNKLTVQRIGKIYAGRDLTFNKSNAGGKSEI INRGT INVKNKLS GYSEGDLNIKGKFADLDNKLTVQRIGKIYAGRDLTFNKSNAGGKSEI INRGTINVKNKLS GYSEGDLNIKGKF ADLDNKLTVORIGKIYAGRDLTFNKSNAGGKSEI INRGTINVKNKLS GYSEGDLNIKGKFADLDNKLTVQRIGKIYAGRDLTFNKSNAGGKSEI INRGTINVKNKLS 
NZV1

82-029362

6644

HD 183

HMC 46

HMC 56

$35000 \mathrm{HP}$

NZS 1

NZS2

NZS 3

NZS 4

NZV1

82-029362

6644

HD 183

HMC 46

HMC 56

$35000 \mathrm{HP}$

NZS 1

NZS2

NZS 3

NZS 4

NZV1

82-029362

6644

HD 183

HMC 46

HMC 56

$35000 \mathrm{HP}$

NZS 1

NZS2

NZS 3

NZS4

NZV1

82-029362

6644

HD 183

HMC 46

HMC 56

$35000 \mathrm{HP}$

NZS 1

NZS2

NZS 3

NZS 4

NZV1

82-029362

6644

HD 183

HMC 46

HMC 56

$35000 \mathrm{HP}$

NZS 1

NZS2

NZS 3

NZS 4

NZV1

82-029362

6644

HD 183

HMC 46

HMC 56
GYSEGDLNIKGKF ADLDNKLTVQRIGKIYAGRDLTFNKSNAGGKSEI INRGTINVKNKLS GYSEGDLNIKGKF ADLDNKLTVQRIGKIYAGRDLTFNKSNAGGKSEI INRGT INVKNKLS GYSEGDLNIKGKF ADLDNKLTVQRIGKIYAGRDLTFNKSNAGGKSE I INRGTINVKNKLS GYSEGDLNI KGKFADLDNKLTVORIGKIYAGRDLTFNKSNAGGKSEI INRGT INVKNKLS GYSEGDLNIKGKF ADLDNKLTVQRIGKIYAGRDLTFNKSNAGGKSEI INRGTINVKNKLS GYSEGDLNI KGKFADLDNKLTVQRIGKIYAGRDLTFNKSNAGGKSEI INRGTINVKNKLS $* * * * * * * * * * * * * * * * * * * * * * * * * * * * * * * * * * * * * * * * * * * * * * * * * * * * * * * * * * * *$

YDSDVSFENNMOSOKVDLYTKIFEAKSDIELTFKTNGTHPVYLNFKSNNNEKKYRNSENT YDSDVSFENNMQSQKVDLYTKIFEAKSDIELTFKTNGTHPVYLNFKSNNNEKKYRNSENT YDSDVSFENNMOSOKVDLYTKIFEAKSDIELTFKTNGTHPVYLNFKSNNNEKKYRNSENT YDSDVSFENNMQSQKVDLYTKIFEAKSDIELTFKTNGTHPVYLNFKSNNNEKKYRNSENT YDSDVSFENNMOSOKVDLYTKIFEAKSDIELTFKTNGTHPVYLNFKSNNNEKKYRNSENT YDSDVSFENNMOSOKVDLYTKIFEAKSDIELTFKTNGTHPVYLNFKSNNNEKKYRNSENT YDSDVSFENNMOSQKVDLYTKIFEAKSDIELTFKTNGTHPVYLNFKSNNNEKKYRNSENT YDSDVSFENNMOSOKVDLYTKIFEAKSDIELTFKTNGTHPVYLNFKSNNNEKKYRNSENT YDSDVSFENNMOSOKVDLYTKIFEAKSDIELTFKTNGTHPVYLNFKSNNNEKKYRNSENT YDSDVSFENNMQSQKVDLYTKIFEAKSDIELTFKTNGTHPVYLNFKSNNNEKKYRNSENT YDSDVSFENNMQSQKVDLYTKIFEAKSDIELTFKTNGTHPVYLNFKSNNNEKKYRNSENT $* * * * * * * * * * * * * * * * * * * * * * * * * * * * * * * * * * * * * * * * * * * * * * * * * * * * * * * * * * * *$

KNFKSIGDLINEALSDSAPEAIEAYYSGSSSNYINPVSYLAALGNANNSSNPHYLNTALK KNFKSIGDLINEALSDSAPEAIEAYYSGSSSNY INPVSYLAALGNANNSSNPHYLNTALK KNFKSIGDLINEALSDSAPEAIEAYYSGSSSNYINPVSYLAALGNANNSSNPHYLNTALK KNFKSIGDLINEALSDSAPEAIEAYYSGSSSNYINPVSYLAALGNANNSSNPHYLNTALK KNFKSIGDLINEALSDSAPEAIEAYYSGSSSNYINPVSYLAALGNANNSSNPHYLNTALK KNFKSIGDLINEALSDSAPEAIEAYYSGSSSNYINPVSYLAALGNANNSSNPHYLNTALK KNFKSIGDLINEALSDSAPEAIEAYYSGSSSNCINPVSYLAALGNANNSSNPHYLNTALK KNFKSIGDLINEALSDSAPEAIEAYYSGSSSNCINPVSYLAALGNANNSSNPHYLNTALK KNFKSIGDLINEALSDSAPEAIEAYYSGSSSNYINPVSYLAALGNANNSSNPHYLNTALK KNFKSIGDLINEALSDSAPEAIEAYYSGSSSNCINPVSYLAALGNANNSSNPHYLNTALK KNFKSIGDLINEALSDSAPEAIEAYYSGSSSNCINPVSYLAALGNANNSSNPHYLNTALK

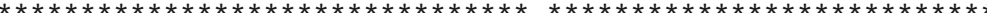

HILGNGWQDDLKKQEN I KVLKOKWEDFKKDKGASKMLDLYPNTDKEKAKIFAGI IRNGND HILGNGWQDDLKKQEN I KVLKQKWEDFKKDKGASKMLDLYPNTDKEKAKIFAGI IRNGND HILGNGWQDDLKKQENIKVLKQKWEDFKKDKGASKMLDLYPNTDKEKAKIFAGIIRNGND HILGNGWQDDLKKQEN I KVLKQKWEDFKKDKGASKMLDLYPNTDKEKAKIFAGI IRNGND HILGNGWQDDLKKQEN I KVLKQKWEDFKKDKGASKMLDLYPNTDKEKAKIFAGI IRNGND HILGNGWODDLKKOENIKVLKOKWEDFKKDKGASKMLDLYPNTDKEKAKIFAGIIRNGND HILGNGWQDDLKKQENIKVLKQKWEDFKKDKGASKMLDLYPNTDKEKAKIFAGIIRNGND HILGNGWQDDLKKQENIKVLKQKWEDFKKDKGASKMLDLYPNTDKEKAKIFAGIIRNGND HILGNGWODDLKKOENIKVLKOKWEDFKKDKGASKMLDLYPNTDKEKAKIFAGIIRNGND HILGNGWQDDLKKQENIKVLKOKWEDFKKDKGASKMLDLYPNTDKEKAKIFAGI IRNGND HILGNGWQDDLKKQENIKVLKQKWEDFKKDKGASKMLDLYPNTDKEKAKIFAGI IRNGND $* * * * * * * * * * * * * * * * * * * * * * * * * * * * * * * * * * * * * * * * * * * * * * * * * * * * * * * * * * * *$

TISDVESEDFKKKYSKFQNGEWAKNDTGTDSYDSTKASEKYKKVENVDHKENIDEHKLNI TISDVESEDFKKKYSKFONGEWAKNDTGTDSYDSTKASEKYKKVENVDHKENIDEHKLNI TISDVESEDFKKKYSKFQNGEWAKNDTGTDSYDSTKASEKYKKVENVDHKENIDEHKLNI TISDVESEDFKKKYSKFQNGEWAKNDTGTDSYDSTKASEKYKKVENVDHKENIDEHKLNI TISDVESEDFKKKYSKFONGEWAKNDTGTDSYDSTKASEKYKKVENVDHKENIDEHKLNI TISDVESEDFKKKYSKFQNGEWAKNDTGTDSYDSTKASEKYKKVENVDHKENIDEHKLNI TISDVESEDFKKKYSKFQNGEWAKNDTGTDSYDSTKASEKYKKVENVDHKENIDEHKLNI TISDVESEDFKKKYSKFONGEWAKNDTGTDSYDSTKASEKYKKVENVDHKENIDEHKLNI TISDVESEDFKKKYSKFQNGEWAKNDTGTDSYDSTKASEKYKKVENVDHKENIDEHKLNI TISDVESEDFKKKYSKFONGEWAKNDTGTDSYDSTKASEKYKKVENVDHKENIDEHKLNI TISDVESEDFKKKYSKFQNGEWAKNDTGTDSYDSTKASEKYKKVENVDHKENIDEHKLNI $* * * * * * * * * * * * * * * * * * * * * * * * * * * * * * * * * * * * * * * * * * * * * * * * * * * * * * * * * * * *$

GKHEITVPGVSFENLNNKNMDHQPDKLGEIDKS I ISELLAQPVYTEKSAARDSDPRVNQN GKHEITVPGVSFENLNNKNMDHQPDKLGEIDKS I ISELLAQPVYTEKSAARDSDPRVNQN GKHEITVPGVSFENLNNKNMDHOPDKLGEIDKSI ISELLAQPVYTEKSAARDSDPRVNQN GKHEITVPGVSFENLNNKNMDHQPDKLGEIDKS I ISELLAQPVYTEKSAARDSDPRVNQN GKHEITVPGVSFENLNNKNMDHQPDKLGEIDKS I ISELLAQPVYTEKSAARDSDPRVNQN GKHEITVPGVSFENLNNKNMDHQPDKLGEIDKSI ISELLAQPVYTEKSAARDSDPRVNQN GKHEITVPGVSFENLNNKNMDHQPDKLGEIDKS I ISELLAQPVYTEKSAARDSDPRVNQN GKHEITVPGVSFENLNNKNMDHOPDKLGEIDKS I ISELLAQPVYTEKSAARDSDPRVNQN GKHEITVPGVSFENLNNKNMDHOPDKLGEIDKS I ISELLAOPVYTEKSAARDSDPRVNON GKHEITVPGVSFENLNNKNMDHQPDKLGEIDKSI I SELLAQPVYTEKSAARDSDPRVNQN GKHEITVPGVSFENLNNKNMDHQPDKLGE IDKS I ISELLAQPVYTEKSAARDSDPRVNQN $* * * * * * * * * * * * * * * * * * * * * * * * * * * * * * * * * * * * * * * * * * * * * * * * * * * * * * * * * * * *$ 
$35000 \mathrm{HP}$

NZS 1

NZS2

NZS3

NZS4

NZV1

82-029362

6644

HD 183

HMC 46

HMC 56

$35000 \mathrm{HP}$

NZS 1

NZS2

NZS 3

NZS 4

NZV1

82-029362

6644

HD 183

HMC 46

HMC 56

$35000 \mathrm{HP}$

NZS 1

NZS2

NZS 3

NZS 4

NZV1

82-029362

6644

HD 183

HMC 46

HMC 56

$35000 \mathrm{HP}$

NZS 1

NZS2

NZS 3

NZS4

NZV1

82-029362

6644

HD 183

HMC 46

HMC 56

$35000 \mathrm{HP}$

NZS 1

NZS2

NZS 3

NZS 4

NZV1

82-029362

6644

HD 183

HMC 46

HMC 56

$35000 \mathrm{HP}$

NZS 1

NZS2

NZS3

NZS 4

NZV1

82-029362

6644
DKEALDNLYRTRLSY INONNYLGAKYFFNOLDTEDDKLKGIKRIGDNYFEHOLITRLIEK DKEALDNLYRTRLSYINQNNYLGAKYFFNQLDTEDDKLKGIKRIGDNYFEHQLITRLIEK DKEALDNLYRTRLSY INQNNYLGAKYFFNQLDTEDDKLKGIKRIGDNYFEHQLITRLIEK DKEALDNLYRTRLSY INQNNYLGAKYFFNQLDTEDDKLKGIKRIGDNYFEHOLITRLIEK DKEALDNLYRTRLSY INQNNYLGAKYFFNQLDTEDDKLKGIKRIGDNYFEHQLITRLIEK DKEALDNLYRTRLSY INQNNYLGAKYFFNQLDTEDDKLKGIKRIGDNYFEHQLITRLIEK DKEALDNLYRTRLSYINQNNYLGAKYFFNQLDTEDDKLKGIKRIGDNYFEYQLITRLIEK DKEALDNLYRTRLSY INQNNYLGAKYFFNQLDTEDDKLKGIKRIGDNYFEYQLITRLIEK DKEALDNLYRTRLSYINQNNYLGAKYFFNOLDTEDDKLKGIKRIGDNYFEHOLITRLIEK DKEALDNLYRTRLSY INQNNYLGAKYFFNQLDTEDDKLKGIKRIGDNYFEYQLITRLIEK DKEALDNLYRTRLSY INQNNYLGAKYFFNQLDTEDDKLKGIKRIGDNYFEYQLITRLIEK $* * * * * * * * * * * * * * * * * * * * * * * * * * * * * * * * * * * * * * * * * * * * * * * * * * *: * * * * * * * * *$

VADNHLTLKHGLHDIALVKKLIDSASIQAKDLNLKVGEALTKEQKDNLKEDIVWYVKTEV VADNHLTLKHGLHDIALVKKLIDSASIQAKDLNLKVGEALTKEQKDNLKEDIVWYVKTEV VADNHLTLKHGLHDIALVKKLIDSASIOAKDLNLKVGEALTKEOKDNLKEDIVWYVKTEV VADNHLTLKHGLHDIALVKKLIDSAS IQAKDLNLKVGEALTKEQKDNLKEDIVWYVKTEV VADNHLTLKHGLHDIALVKKLIDSASIQAKDLNLKVGEALTKEQKDNLKEDIVWYVKTEV VADNHLTLKHGLHDIALVKKLIDSASIOAKDLNLKVGEALTKEOKDNLKEDIVWYVKTEV VADNHLTLKHGLHDIALVKKLIDSASIQAKDLNLKVGEALTKEQKDNLKEDIVWYVKTEV VADNHLTLKHGLHDIALVKKLIDSAS IOAKDLNLKVGEALTKEOKDNLKEDIVWYVKTEV VADNHLTLKHGLHDIALVKKLIDSAS IQAKDLNLKVGEALTKEQKDNLKEDIVWYVKTEV VADNHLTLKHGLHDIALVKKLIDSAS IQAKDLNLKVGEALTKEQKDNLKEDIVWYVKTEV VADNHLTLKHGLHDIALVKKLIDSASIQAKDLNLKVGEALTKEQKDNLKEDIVWYVKTEV $\star * * * * * * * * * * * * * * * * * * * * * * * * * * * * * * * * * * * * * * * * * * * * * * * * * * * * * * * * * * * * * * * * * *$

NGQEVLVPQVYLAKQTIEEVEKQRGVGTGQIRAGI IDVKVDDVRNTGTIAGYAVGLEAKN NGOEVLVPOVYLAKOTIEEVEKORGVGTGOIRAGI IDVKVDDVRNTGTIAGYAVGLEAKN NGQEVLVPQVYLAKQTIEEVEKQRGVGTGQIRAGI IDVKVDDVRNTGTIAGYAVGLEAKN NGQEVLVPQVYLAKQTIEEVEKQRGVGTGQIRAGI IDVKVDDVRNTGTIAGYAVGLEAKN NGQEVLVPOVYLAKOTIEEVEKORGVGTGOIRAGI IDVKVDDVRNTGTIAGYAVGLEAKN NGQEVLVPQVYLAKQTIEEVEKQRGVGTGQIRAGI IDVKVDDVRNTGTIAGYAVGLEAKN NGQEVLVPQVYLAKQTIEEVEKQRGVGTGQIRAGI IDVKVDDVRNTGTIAGYAVGLEAKN NGQEVLVPQVYLAKQTIEEVEKQRGVGTGQIRAGI IDVKVDDVRNTGTIAGYAVGLEAKN NGQEVLVPQVYLAKQTIEEVEKQRGVGTGQIRAGI IDVKVDDVRNTGTIAGYAVGLEAKN NGQEVLVPQVYLAKQTIEEVEKORGVGTGQIRAGI IDVKVDDVRNTGTIAGYAVGLEAKN NGQEVLVPQVYLAKQTIEEVEKQRGVGTGQIRAGI IDVKVDDVRNTGTIAGYAVGLEAKN

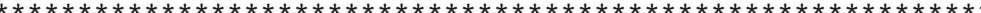

KLKNTGDILSORLSKLVGKKGLESTGVTYVDETGATKVRKARIKSEGHIYLETDKDKNVD KLKNTGDILSQRLSKLVGKKGLESTGVTYVDETGATKVRKARIKSEGHIYLETDKDKNVD KLKNTGDILSQRLSKLVGKKGLESTGVTYVDETGATKVRKARIKSEGHIYLETDKDKNVD KLKNTGDILSORLSKLVGKKGLESTGVTYVDETGATKVRKARIKSEGHIYLETDKDKNVD KLKNTGDILSQRLSKLVGKKGLESTGVTYVDETGATKVRKARIKSEGHIYLETDKDKNVD KLKNTGDILSORLSKLVGKKGLESTGVTYVDETGATKVRKARIKSEGHIYLETDKDKNVD KLKNTGDILSQRLSKLVGKKGLESTGVTYVDETGATKVRKARIKSEGHIYLETDKDKNVD KLKNTGDILSORLSKLVGKKGLESTGVTYVDETGATKVRKARIKSEGHIYLETDKDKNVD KLKNTGDILSORLSKLVGKKGLESTGVTYVDETGATKVRKARIKSEGHIYLETDKDKNVD KLKNTGDILSQRLSKLVGKKGLESTGVTYVDETGATKVRKARIKSEGHIYLETDKDKNVD KLKNTGDILSORLSKLVGKKGLESTGVTYVDETGATKVRKARIKSEGHIYLETDKDKNVD $* * * * * * * * * * * * * * * * * * * * * * * * * * * * * * * * * * * * * * * * * * * * * * * * * * * * * * * * * * * *$

LTASELKGNTGQIKAKDLNLNDIYETSYKYKYEKLFGKNGGEIGDRVTQTSQAKSVGTDA LTASELKGNTGQIKAKDLNLNDIYETSYKYKYEKLFGKNGGEIGDRVTQTSQAKSVGTDA LTASELKGNTGQIKAKDLNLNDIYETSYKYKYEKLFGKNGGEIGDRVTQTSQAKSVGTDA LTASELKGNTGQIKAKDLNLNDIYETSYKYKYEKLFGKNGGEIGDRVTQTSQAKSVGTDA LTASELKGNTGOIKAKDLNLNDIYETSYKYKYEKLFGKNGGEIGDRVTOTSOAKSVGTDA LTASELKGNTGQIKAKDLNLNDIYETSYKYKYEKLFGKNGGEIGDRVTQTSQAKSVGTDA LTASELKGSTGQIKAKDLNLNDIYETSYKYKYEKLFGKNGGEIGDRVTQTSQAKSVGTDA LTASELKGSTGQIKAKDLNLNDIYETSYKYKYEKLFGKNGGEIGDRVTOTSOAKSVGTDA LTASELKGNTGQIKAKDLNLNDIYETSYKYKYEKLFGKNGGE I GDRVTQTSQAKSVGTDA LTASELKGSTGQIKAKDLNLNDIYETSYKYKYEKLFGKNGGEIGDRVTQTSQAKSVGTDA LTASELKGSTGQIKAKDLNLNDIYETSYKYKYEKLFGKNGGEIGDRVTQTSQAKSVGTDA $* * * * * * * * . * * * * * * * * * * * * * * * * * * * * * * * * * * * * * * * * * * * * * * * * * * * * * * * * * * * * * * * *$

SFDHLHLSLEGDVNQTGSNLKANRTTGVVKGDFNTKAGKDLFHRQIDTVTSGTVYSASAS SFDHLHLSLEGDVNQTGSNLKANRTTGVVKGDFNTKAGKDLFHRQIDTVTSGTVYSASAS SFDHLHLSLEGDVNQTGSNLKANRTTGVVKGDFNTKAGKDLFHRQIDTVTSGTVYSASAS SFDHLHLSLEGDVNOTGSNLKANRTTGVVKGDFNTKAGKDLFHROIDTVTSGTVYSASAS SFDHLHLSLEGDVNQTGSNLKANRTTGVVKGDFNTKAGKDLFHRQIDTVTSGTVYSASAS SFDHLHLSLEGDVNQTGSNLKANRTTGVVKGDFNTKAGKDLFHRQIDTVTSGTVYSASAS SFDHLHLSLEGDVNOTGSNLKANRTTGVVKGDFNTKAGKDLFHROIDTVTSGTVYSASAS SFDHLHLSLEGDVNQTGSNLKANRTTGVVKGDFNTKAGKDLFHRQIDTVTSGTVYSASAS 
HD183

HMC 46

HMC 56

$35000 \mathrm{HP}$

NZS 1

NZS2

NZS 3

NZS4

NZV1

82-029362

6644

HD 183

HMC 46

HMC 56

$35000 \mathrm{HP}$

NZS 1

NZS2

NZS 3

NZS 4

NZV1

82-029362

6644

HD 183

HMC 46

HMC 56

$35000 \mathrm{HP}$

NZS 1

NZS2

NZS 3

NZS4

NZV1

82-029362

6644

HD 183

HMC 46

HMC 56

$35000 \mathrm{HP}$

NZS 1

NZS2

NZS 3

NZS 4

NZV1

$82-029362$

6644

HD 183

HMC 46

HMC 56

$35000 \mathrm{HP}$

NZ 1

NZS2

NZS 3

NZS 4

NZV1

82-029362

6644

HD 183

HMC 46

HMC 56

$35000 \mathrm{HP}$

NZS 1

NZS2
SFDHLHLSLEGDVNQTGSNLKANRTTGVVKGDFNTKAGKDLFHRQIDTVTSGTVYSASAS SFDHLHLSLEGDVNQTGSNLKANRTTGVVKGDFNTKAGKDLFHRQIDTVTSGTVYSASAS SFDHLHLSLEGDVNQTGSNLKANRTTGVVKGDFNTKAGKDLFHRQIDTVTSGTVYSASAS

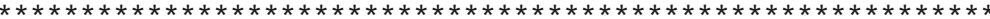

GGGOSAGISLTDQGVETYTNKTATAGANADVTNFMKRTRETETSLTHRNSEFNALSGELY GGGQSAGISLTDQGVETYTNKTATAGANADVTNFMKRTRETETSLTHRNSEFNALSGELY GGGOSAGISLTDQGVETYTNKTATAGANADVTNFMKRTRETETSLTHRNSEFNALSGELY GGGQSAGISLTDQGVETYTNKTATAGANADVTNFMKRTRETETSLTHRNSEFNALSGELY GGGQSAGISLTDQGVETYTNKTATAGANADVTNFMKRTRETETSLTHRNSEFNALSGELY GGGOSAGISLTDOGVETYTNKTATAGANADVTNFMKRTRETETSLTHRNSEFNALSGELY GGGQSAGISLTDQGVETYTNKTATAGANADVTNFMKRTRETETSLTHRNSEFNALSGELY GGGQSAGISLTDQGVETYTNKTATAGANADVTNFMKRTRETETSLTHRNSEFNALSGELY GGGQSAGISLTDQGVETYTNKTATAGANADVTNFMKRTRETETSLTHRNSEFNALSGELY GGGQSAGISLTDQGVETYTNKTATAGANADVTNFMKRTRETETSLTHRNSEFNALSGELY GGGQSAGISLTDQGVETYTNKTATAGANADVTNFMKRTRETETSLTHRNSEFNALSGELY $* * * * * * * * * * * * * * * * * * * * * * * * * * * * * * * * * * * * * * * * * * * * * * * * * * * * * * * * * * * *$

VMGKADIGGVDINRDVEVIKTPEEIAAEOKAAEEAKKAEVKENEASETAAKETEEAENDN VMGKADIGGVDINRDVEVIKTPEEIAAEQKAAEEAKKAEVKENEASETAAKETEEAENDN VMGKADIGGVDINRDVEVIKTPEEIAAEQKAAEEAKKAEVKENEASETAAKETEEAENDN VMGKADIGGVDINRDVEVIKTPEEIAAEQKAAEEAKKAEVKENEASETAAKETEEAENDN VMGKADIGGVDINRDVEVIKTPEEIAAEQKAAEEAKKAEVKENEASETAAKETEEAENDN VMGKADIGGVDINRDVEVIKTPEEIAAEQKAAEEAKKAEVKENEASETAAKETEEAENDN VMGKADIGGVDINRDVEVIKTPEEIAAEQKAAEEAKKAEVKENEASETAAKETEEAENDN VMGKADIGGVDINRDVEVIKTPEEIAAEQKAAEEAKKAEVKENEASETAAKETEEAENDN VMGKADIGGVDINRDVEVIKTPEEIAAEQKAAEEAKKAEVKENEASETAAKETEEAENDN VMGKADIGGVDINRDVEVIKTPEEIAAEOKAAEEAKKAEVKENEASETAAKETEEAENDN VMGKADIGGVDINRDVEVIKTPEE IAAEQKAAEEAKKAEVKENEASETAAKETEEAENDN $* * * * * * * * * * * * * * * * * * * * * * * * * * * * * * * * * * * * * * * * * * * * * * * * * * * * * * * * * * * *$

VAEKDKTKPKFKKLTDEEIAAAFETKGEDFFAAYKAREEEDRKKGFTLSAEQIESTKARD VAEKDKTKPKFKKLTDEE IAAAFETKGEDFFAAYKAREEEDRKKGFTLSAEQIESTKARD VAEKDKTKPKFKKLTDEEIAAAFETKGEDFFAAYKAREEEDRKKGFTLSAEQIESTKARD VAEKDKTKPKFKKLTDEEIAAAFETKGEDFFAAYKAREEEDRKKGFTLSAEQIESTKARD VAEKDKTKPKFKKLTDEEIAAAFETKGEDFFAAYKAREEEDRKKGFTLSAEQIESTKARD VAEKDKTKPKFKKLTDEEIAAAFETKGEDFFAAYKAREEEDRKKGFTLSAEQIESTKARD VAEKDKTKPKFKKLTDEEIAAAFETKGEDFFAAYKAREEEDRKKGFTLSAEQIESTKARD VAEKDKTKPKFKKLTDEEIAAAFETKGEDFFAAYKAREEEDRKKGFTLSAEQIESTKARD VAEKDKTKPKFKKLTDEEIAAAFETKGEDFFAAYKAREEEDRKKGFTLSAEOIESTKARD VAEKDKTKPKFKKLTDEEIAAAFETKGEDFFAAYKAREEEDRKKGFTLSAEQIESTKARD VAEKDKTKPKFKKLTDEEIAAAFETKGEDFFAAYKAREEEDRKKGFTLSAEQIESTKARD $* * * * * * * * * * * * * * * * * * * * * * * * * * * * * * * * * * * * * * * * * * * * * * * * * * * * * * * * * * * * * * *$

EKETTYYELKVGVGAEAEAHSAAADAISNKAROI IDTONGLKODGTVALOEASDVLNLAT EKETTYYELKVGVGAEAEAHSAAADAISNKAROI IDTQNGLKODGTVALQEASDVLNLAT EKETTYYELKVGVGAEAEAHSAAADAISNKARQI IDTQNGLKQDGTVALQEASDVLNLAT EKETTYYELKVGVGAEAEAHSAAADAISNKARQI IDTQNGLKQDGTVALQEASDVLNLAT EKETTYYELKVGVGAEAEAHSAAADAISNKARQI IDTQNGLKQDGTVALQEASDVLNLAT EKETTYYELKVGVGAEAEAHSAAADAISNKARQI IDTQNGLKODGTVALQEASDVLNLAT EKETTYYELKVGVGAEAEAHSAAADAISNKARQI IDTQNGLKODGTVALQEASDVLNLAT EKETTYYELKVGVGAEAEAHSAAADAISNKARQI IDTQNGLKODGTVALQEASDVLNLAT EKETTYYELKVGVGAEAEAHSAAADAISNKARQI IDTQNGLKQDGTVALQEASDVLNLAT EKETTYYELKVGVGAEAEAHSAAADAISNKARQI IDTQNGLKQDGTVALQEASDVLNLAT EKETTYYELKVGVGAEAEAHSAAADAISNKARQI IDTONGLKODGTVALQEASDVLNLAT $* * * * * * * * * * * * * * * * * * * * * * * * * * * * * * * * * * * * * * * * * * * * * * * * * * * * * * * * * * * *$

GDLAGASAKLKFELSTIEKKSRGASDGRSILGGRLNLAARGGDITLNNVETTENSHLSLK GDLAGASAKLKFELSTIEKKSRGASDGRSILGGRLNLAARGGDITLNNVETTENSHLSLK GDLAGASAKLKFELSTIEKKSRGASDGRS ILGGRLNLAARGGDITLNNVETTENSHLSLK GDLAGASAKLKFELSTIEKKSRGASDGRS ILGGRLNLAARGGDITLNNVETTENSHLSLK GDLAGASAKLKFELSTIEKKSRGASDGRS ILGGRLNLAARGGDITLNNVETTENSHLSLK GDLAGASAKLKFELSTIEKKSRGASDGRSILGGRLNLAARGGDITLNNVETTENSHLSLK GDLAGASAKLKFELSTIEKKSRGASDGRS ILGGRLNLAARGGDITLNNVETTENSHLSLK GDLAGASAKLKFELSTIEKKSRGASDGRS ILGGRLNLAARGGDITLNNVETTENSHLSLK GDLAGASAKLKFELSTIEKKSRGASDGRS ILGGRLNLAARGGDITLNNVETTENSHLSLK GDLAGASAKLKFELSTIEKKSRGASDGRS ILGGRLNLAARGGDITLNNVETTENSHLSLK GDLAGASAKLKFELSTIEKKSRGASDGRSILGGRLNLAARGGDITLNNVETTENSHLSLK

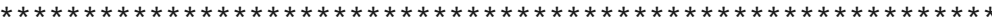

ARDNVNVNSGVTEQKDESNSQSLKVTAGASSGCGVMAGGCSAGVSAGVSGSYNESNTEST ARDNVNVNSGVTEQKDESNSOSLKVTAGASSGCGVMAGGCSAGVSAGVSGSYNESNTEST ARDNVNVNSGVTEQKDESNSQSLKVTAGASSGCGVMAGGCSAGVSAGVSGSYNESNTEST 
NZS 3

NZS 4

NZV1

82-029362

6644

HD 183

HMC 46

HMC 56

$35000 \mathrm{HP}$

NZS 1

NZS2

NZS3

NZS4

NZV1

82-029362

6644

HD 183

HMC 46

HMC 56

$35000 \mathrm{HP}$

NZS 1

NZS2

NZS 3

NZS 4

NZV1

82-029362

6644

HD 183

HMC 46

HMC 56

$35000 \mathrm{HP}$

NZS 1

NZS2

NZS 3

NZS 4

NZV1

82-029362

6644

HD 183

HMC 46

HMC 56

$35000 \mathrm{HP}$

NZS 1

NZS2

NZS 3

NZS4

NZV1

82-029362

6644

HD 183

HMC 46

HMC 56

$35000 \mathrm{HP}$

NZS 1

NZS2

NZS 3

NZS 4

NZV1

82-029362

6644

HD 183

HMC 46

HMC 56
ARDNVNVNSGVTEQKDESNSQSLKVTAGASSGCGVMAGGCSAGVSAGVSGSYNESNTEST ARDNVNVNSGVTEOKDESNSOSLKVTAGASSGCGVMAGGCSAGVSAGVSGSYNESNTEST ARDNVNVNSGVTEQKDESNSQSLKVTAGASSGCGVMAGGCSAGVSAGVSGSYNESNTEST ARDNVNVNSGVTEQKDESNSQSLKVTAGASSGCGVMAGGCSAGVSAGVSGSYNESNTEST ARDNVNVNSGVTEQKDESNSQSLKVTAGASSGCGVMAGGCSAGVSAGVSGSYNESNTEST ARDNVNVNSGVTEQKDESNSQSLKVTAGASSGCGVMAGGCSAGVSAGVSGSYNESNTEST ARDNVNVNSGVTEQKDESNSQSLKVTAGASSGCGVMAGGCSAGVSAGVSGSYNESNTEST ARDNVNVNSGVTEQKDESNSQSLKVTAGASSGCGVMAGGCSAGVSAGVSGSYNESNTEST $* * * * * * * * * * * * * * * * * * * * * * * * * * * * * * * * * * * * * * * * * * * * * * * * * * * * * * * * * * * *$

SHTNSLLRGKSLRVEAGKDFNLISSNVDVDHLHLDVKGDTNVVSKQDSYSRKERGVNYSV SHTNSLLRGKSLRVEAGKDFNLISSNVDVDHLHLDVKGDTNVVSKQDSYSRKERGVNYSV SHTNSLLRGKSLRVEAGKDFNLISSNVDVDHLHLDVKGDTNVVSKQDSYSRKERGVNYSV SHTNSLLRGKSLRVEAGKDFNLISSNVDVDHLHLDVKGDTNVVSKODSYSRKERGVNYSV SHTNSLLRGKSLRVEAGKDFNLISSNVDVDHLHLDVKGDTNVVSKQDSYSRKERGVNYSV SHTNSLLRGKSLRVEAGKDFNLISSNVDVDHLHLDVKGDTNVVSKODSYSRKERGVNYSV SHTNSLLRGKSLRVEAGKDFNLISSNVDVDHLHLDVKGDTNVVSKQDSYSRKERGVNYSV SHTNSLLRGKSLRVEAGKDFNLISSNVDVDHLHLDVKGDTNVVSKQDSYSRKERGVNYSV SHTNSLLRGKSLRVEAGKDFNLISSNVDVDHLHLDVKGDTNVVSKQDSYSRKERGVNYSV SHTNSLLRGKSLRVEAGKDFNLISSNVDVDHLHLDVKGDTNVVSKQDSYSRKERGVNYSV SHTNSLLRGKSLRVEAGKDFNLISSNVDVDHLHLDVKGDTNVVSKQDSYSRKERGVNYSV $* * * * * * * * * * * * * * * * * * * * * * * * * * * * * * * * * * * * * * * * * * * * * * * * * * * * * * * * * * * *$

SAGVGVSTAGGARPNGSVGLGVSAENENSKIVKQQAGISAKRITGE INNLNLTGGYIENK SAGVGVSTAGGARPNGSVGLGVSAENENSKIVKQQAGISAKRITGE INNLNLTGGYIENK SAGVGVSTAGGARPNGSVGLGVSAENENSKIVKQQAGISAKRITGE INNLNLTGGYIENK SAGVGVSTAGGARPNGSVGLGVSAENENSKIVKQQAG ISAKRITGE INNLNLTGGYIENK SAGVGVSTAGGARPNGSVGLGVSAENENSKIVKOOAGISAKRITGE INNLNLTGGY IENK SAGVGVSTAGGARPNGSVGLGVSAENENSKIVKQQAGISAKRITGE INNLNLTGGYIENK SAGVGVSTAGGARPNGSVGLGVSAENENSKIVKQQAGISAKRITGE INNLNLTGGYIENK SAGVGVSTAGGARPNGSVGLGVSAENENSKIVKQQAGISAKRITGE INNLNLTGGY IENK SAGVGVSTAGGARPNGSVGLGVSAENENSKIVKQQAGISAKRITGE INNLNLTGGYIENK SAGVGVSTAGGARPNGSVGLGVSAENENSKIVKQQAGISAKRITGE INNLNLTGGYIENK SAGVGVSTAGGARPNGSVGLGVSAENENSKIVKQQAGISAKRITGE INNLNLTGGYIENK $* * * * * * * * * * * * * * * * * * * * * * * * * * * * * * * * * * * * * * * * * * * * * * * * * * * * * * * * * * * *$

GNPDELNVKGDITTHELKDEHHKDGGSFGGSVGVSETGVTQVNVNGGRVEQKHYEATQHS GNPDELNVKGDITTHELKDEHHKDGGSFGGSVGVSETGVTQVNVNGGRVEQKHYEATQHS GNPDELNVKGDITTHELKDEHHKDGGSFGGSVGVSETGVTQVNVNGGRVEQKHYEATQHS GNPDELNVKGDITTHELKDEHHKDGGSFGGSVGVSETGVTOVNVNGGRVEOKHYEATOHS GNPDELNVKGDITTHELKDEHHKDGGSFGGSVGVSETGVTQVNVNGGRVEQKHYEATQHS GNPDELNVKGDITTHELKDEHHKDGGSFGGSVGVSETGVTQVNVNGGRVEQKHYEATQHS GNPDELNVKGDITTHELKDEHHKDGGSFGGSVGVSETGVTQVNVNGGRVEQKHYEATQHS GNPDELNVKGDITTHELKDEHHKDGGSFGGSVGVSETGVTQVNVNGGRVEQKHYEATQHS GNPDELNVKGDITTHELKDEHHKDGGSFGGSVGVSETGVTQVNVNGGRVEQKHYEATQHS GNPDELNVKGDITTHELKDEHHKDGGSFGGSVGVSETGVTQVNVNGGRVEQKHYEATQHS GNPDELNVKGDITTHELKDEHHKDGGSFGGSVGVSETGVTQVNVNGGRVEQKHYEATQHS $* * * * * * * * * * * * * * * * * * * * * * * * * * * * * * * * * * * * * * * * * * * * * * * * * * * * * * * * * * * *$

SISGINTKGKTVGNFKTDRSOSTEVHRDDTIAATNFNFELGDIAELAKKGKEKWDNRSAK SISGINTKGKTVGNFKTDRSQSTEVHRDDTIAATNFNFELGDIAELAKKGKEKWDNRSAK SISGINTKGKTVGNFKTDRSQSTEVHRDDTIAATNFNFELGDIAELAKKGKEKWDNRSAK SISGINTKGKTVGNFKTDRSQSTEVHRDDTIAATNFNFELGDIAELAKKGKEKWDNRSAK SISGINTKGKTVGNFKTDRSQSTEVHRDDTIAATNFNFELGDIAELAKKGKEKWDNRSAK SISGINTKGKTVGNFKTDRSQSTEVHRDDTIAATNFNFELGDIAELAKKGKEKWDNRSAK SISGINTKGKTVGNFKTDRSQSTEVHRDDTIAATNFNFELGDIAELAKKGKEKWDNRSAK SISGINTKGKTVGNFKTDRSOSTEVHRDDTIAATNFNFELGDIAELAKKGKEKWDNRSAK SISGINTKGKTVGNFKTDRSOSTEVHRDDTIAATNFNFELGDIAELAKKGKEKWDNRSAK SISGINTKGKTVGNFKTDRSQSTEVHRDDTIAATNFNFELGDIAELAKKGKEKWDNRSAK SISGINTKGKTVGNFKTDRSOSTEVHRDDTIAATNFNFELGDIAELAKKGKEKWDNRSAK $\star * * * * * * * * * * * * * * * * * * * * * * * * * * * * * * * * * * * * * * * * * * * * * * * * * * * * * * * * * * *$

TTSSSQDSAHDPRSRSVENGYSELPRFKTADNDAGVDSPRLIKGEAEQAQTLALTKAGND TTSSSQDSAHDPRSRSVENGYSELPRFKTADNDAGVDSPRLIKGEAEQAQTLALTKAGND TTSSSQDSAHDPRSRSVENGYSELPRFKTADNDAGVDSPRLIKGEAEQAQTLALTKAGND TTSSSQDSAHDPRSRSVENGYSELPRFKTADNDAGVDSPRLIKGEAEQAQTLALTKAGND TTSSSQDSAHDPRSRSVENGYSELPRFKTADNDAGVDSPRLIKGEAEQAQTLALTKAGND TTSSSQDSAHDPRSRSVENGYSELPRFKTADNDAGVDSPRLIKGEAEQAQTLALTKAGND TTSSSODSAHDPRSRSVENGYSELPRFKTADNDAGVDSPRLIKGEAEOAOTLALTKAGND TTSSSQDSAHDPRSRSVENGYSELPRFKTADNDAGVDSPRLIKGEAEQAQTLALTKAGND TTSSSQDSAHDPRSRSVENGYSELPRFKTADNDAGVDSPRLIKGEAEQAQTLALTKAGND TTSSSODSAHDPRSRSVENGYSELPRFKTADNDAGVDSPRLIKGEAEQAQTLALTKAGND TTSSSQDSAHDPRSRSVENGYSELPRFKTADNDAGVDSPRLIKGEAEQAQTLALTKAGND 


\section{$35000 \mathrm{HP}$ \\ NZS 1 \\ NZS2 \\ NZS 3 \\ NZS4 \\ NZV1 \\ 82-029362 \\ 6644 \\ HD 183 \\ HMC 46 \\ HMC 56}

$35000 \mathrm{HP}$

NZS 1

NZS2

NZS 3

NZS4

NZV1

82-029362

6644

HD 183

HMC 46

HMC 56

$35000 \mathrm{HP}$

NZS1

NZS2

NZS 3

NZS 4

NZV1

82-029362

6644

HD 183

HMC 46

HMC 56

$35000 \mathrm{HP}$

NZS1

NZS2

NZS3

NZS4

NZV1

82-029362

6644

HD 183

HMC 46

HMC 56

$35000 \mathrm{HP}$

NZS1

NZS2

NZS 3

NZS 4

NZV1

82-029362

6644

HD 183

HMC 46

HMC 56

$35000 \mathrm{HP}$

NZS 1

NZS2

NZS 3

NZS 4

NZV1
$* * * * * * * * * * * * * * * * * * * * * * * * * * * * * * * * * * * * * * * * * * * * * * * * * * * * * * * * * * * *$

VIPEVQSLTQKARPQSLVDESPYAEIPALTRPQVKSNIAESIEVPQFRTKVSDGDEGNYA VIPEVQSLTOKARPQSLVDESPYAEIPALTRPQVKSNIAES IEVPQFRTKVSDGDEGNYA VIPEVQSLTQKARPQSLVDESPYAEIPALTRPQVKSNIAESIEVPQFRTKVSDGDEGNYA VIPEVQSLTQKARPQSLVDESPYAEIPALTRPQVKSNIAES IEVPQFRTKVSDGDEGNYA VIPEVQSLTQKARPQSLVDESPYAEIPALTRPQVKSNIAESIEVPQFRTKVSDGDEGNYA VIPEVQSLTQKARPOSLVDESPYAEIPALTRPQVKSNIAES IEVPQFRTKVSDGDEGNYA VIPEVQSLTQKARPQSLVDESPYAEIPALTRPQVKSNIAES IEVPQFRTKVSDGDEGNYA VIPEVQSLTQKARPQSLVDESPYAEIPALTRPQVKSNIAES IEVPQFRTKVSDGDEGNYA VIPEVOSLTOKARPOSLVDESPYAEIPALTRPOVKSNIAESIEVPOFRTKVSDGDEGNYA VIPEVQSLTQKARPQSLVDESPYAEIPALTRPQVKSNIAESIEVPQFRTKVSDGDEGNYA VIPEVQSLTQKARPQSLVDESPYAEIPALTRPQVKSNIAES IEVPQFRTKVSDGDEGNYA $* * * * * * * * * * * * * * * * * * * * * * * * * * * * * * * * * * * * * * * * * * * * * * * * * * * * * * * * * * * * * * * *$

EITFPTNKAAISSTQDVGDTPTPRALRLESESGYESAENLGLIPRGFKSSPKGEYEDISD EITFPTNKAAISSTQDVGDTPTPRALRLESESGYESAENLGLIPRGFKSSPKGEYEDISD EITFPTNKAAISSTQDVGDTPTPRALRLESESGYESAENLGLIPRGFKSSPKGEYEDISD EITFPTNKAAISSTODVGDTPTPRALRLESESGYESAENLGLIPRGFKSSPKGEYEDISD EITFPTNKAAISSTQDVGDTPTPRALRLESESGYESAENLGLIPRGFKSSPKGEYEDISD EITFPTNKAAISSTQDVGDTPTPRALRLESESGYESAENLGLIPRGFKSSPKGEYEDISD EITFPTNKAAISSTQDVGDTPTPRALRLESESGYESAENLGLIPRGFKSSPKGEYEDISD EITFPTNKAAISSTQDVGDTPTPRALRLESESGYESAENLGLIPRGFKSSPKGEYEDISD EITFPTNKAAISSTQDVGDTPTPRALRLESESGYESAENLGLIPRGFKSSPKGEYEDISD EITFPTNKAAISSTQDVGDTPTPRALRLESESGYESAENLGLIPRGFKSSPKGEYEDISD EITFPTNKAAISSTQDVGDTPTPRALRLESESGYESAENLGLIPRGFKSSPKGEYEDISD $* * * * * * * * * * * * * * * * * * * * * * * * * * * * * * * * * * * * * * * * * * * * * * * * * * * * * * * * * * *$

AIEPQTRSRKLDEPEPIYGTINKSPEAIARANAKADEAIQALGYDPRIKPVVPEEAPPAL AIEPQTRSRKLDEPEPIYGTINKSPEAIARANAKADEAIQALGYDPRIKPVVPEEAPPAL AIEPQTRSRKLDEPEPIYGTINKSPEAIARANAKADEAIQALGYDPRIKPVVPEEAPPAL AIEPQTRSRKLDEPEPIYGTINKSPEAIARANAKADEAIQALGYDPRIKPVVPEEAPPAL AIEPQTRSRKLDEPEPIYGTINKSPEAIARANAKADEAIQALGYDPRIKPVVPEEAPPAI AIEPQTRSRKLDEPEPIYGTINKSPEAIARANAKADEAIQALGYDPRIKPVVPEEAPPAL AIEPQTRSRKLDEPEPIYGTINKSPEAIARANAKADEAIQALGYDPRIKPVVPEEAPPAL AIEPQTRSRKLDEPEPIYGTINKSPEAIARANAKADEAIQALGYDPRIKPVVPEEAPPAL AIEPQTRSRKLDEPEPIYGTINKSPEAIARANAKADEAIQALGYDPRIKPVVPEEAPPAL AIEPQTRSRKLDEPEPIYGTINKSPEAIARANAKADEAIQALGYDPRIKPVVPEEAPPAL AIEPQTRSRKLDEPEPIYGTINKSPEAIARANAKADEAIQALGYDPRIKPVVPEEAPPAL $* * * * * * * * * * * * * * * * * * * * * * * * * * * * * * * * * * * * * * * * * * * * * * * * * * * * * * * * * * * *$

PPRNLQTKAISDYDDVSYVPDFKVRKTDEPEPIYGTINKSPEAIARANAKADEAIQASGY PPRNLQTKAISDYDDVSYVPDFKVRKTDEPEPIYGTINKSPEAIARANAKADEAIQASGY PPRNLQTKAISDYDDVSYVPDFKVRKTDEPEPIYGTINKSPEAIARANAKADEAIQASGY PPRNLQTKAISDYDDVSYVPDFKVRKTDEPEPIYGTINKSPEAIARANAKADEAIQASGY PPRNLQTKAISDYDDVSYVPDFKVRKTDEPEPIYGTINKSPEAIARANAKADEAIQASGY PPRNLQTKAISDYDDVSYVPDFKVRKTDEPEPIYGTINKSPEAIARANAKADEAIQASGY PPRNLQTKAISDYDDVSYVPDFKVRKTDEPEPIYGTINKSPEAIARANAKADEAIQASGY PPRNLQTKAISDYDDVSYVPDFKVRKTDEPEPIYGTINKSPEAIARANAKADEAIQASGY PPRNLQTKAISDYDDVSYVPDFKVRKTDEPEPIYGTINKSPEAIARANAKADEAIQASGY PPRNLQTKAISDYDDVSYVPDFKVRKTDEPEPIYGTINKSPEAIARANAKADEAIQASGY PPRNLQTKAISDYDDVSYVPDFKVRKTDEPEPIYGTINKSPEAIARANAKADEAIQASGY $* * * * * * * * * * * * * * * * * * * * * * * * * * * * * * * * * * * * * * * * * * * * * * * * * * * * * * * * * * * *$

DPRIKPVVPEDAPPALPPRTOSLIDSTEVPSYRSALANVKFDDASPWPOPSALRSKAFAD DPRIKPVVPEDAPPALPPRTQSLIDSTEVPSYRSALANVKFDDASPWPQPSALRSKAFAD DPRIKPVVPEDAPPALPPRTOSLIDSTEVPSYRSALANVKFDDASPWPOPSALRSKAFAD DPRIKPVVPEDAPPALPPRTQSLIDSTEVPSYRSALANVKFDDASPWPQPSALRSKAFAD DPRIKPVVPEDAPPALPPRTQSLIDSTEVPSYRSALANVKFDDASPWPQPSALRSKAFAD DPRIKPVVPEDAPPALPPRTOSLIDSTEVPSYRSALANVKFDDASPWPOPSALRSKAFAD DPRIKPVVPEDAPPALPPRTQSLIDSTEVPSYRSALANVKFDDASPWPQPSALRSKAFAD DPRIKPVVPEDAPPALPPRTQSLIDSTEVPSYRSALANVKFDDASPWPQPSALRSKAFAD DPRIKPVVPEDAPPALPPRTQSLIDSTEVPSYRSALANVKFDDASPWPQPSALRSKAFAD DPRIKPVVPEDAPPALPPRTQSLIDSTEVPSYRSALANVKFDDASPWPQPSALRSKAFAD DPRIKPVVPEDAPPALPPRTQSLIDSTEVPSYRSALANVKFDDASPWPQPSALRSKAFAD $* * * * * * * * * * * * * * * * * * * * * * * * * * * * * * * * * * * * * * * * * * * * * * * * * * * * * * * * * * * *$

EPSSETPKSRGKRGISEESLSSTVQPRSRKISEEDSSFERLPLRIIDNGSDYAEILPRNV EPSSETPKSRGKRGISEESLSSTVOPRSRKISEEDSSFERLPLRIIDNGSDYAEILPRNV EPSSETPKSRGKRGISEESLSSTVQPRSRKISEEDSSFERLPLRI IDNGSDYAEILPRNV EPSSETPKSRGKRGISEESLSSTVQPRSRKISEEDSSFERLPLRIIDNGSDYAEILPRNV EPSSETPKSRGKRGISEESLSSTVOPRSRKISEEDSSFERLPLRIIDNGSDYAEILPRNV EPSSETPKSRGKRGISEESLSSTVQPRSRKISEEDSSFERLPLRIIDNGSDYAEILPRNV 
$82-029362$

6644

HD 183

HMC 46

HMC 56

$35000 \mathrm{HP}$

NZS 1

NZS2

NZS 3

NZS 4

NZV1

82-029362

6644

HD 183

HMC 46

HMC 56

$35000 \mathrm{HP}$

NZS 1

NZS2

NZS 3

NZS4

NZV1

82-029362

6644

HD 183

HMC 46

HMC 56

$35000 \mathrm{HP}$

NZ 1

NZS2

NZS3

NZS 4

NZV1

82-029362

6644

HD 183

HMC 46

HMC 56

$35000 \mathrm{HP}$

NZS 1

NZS2

NZS3

NZS4

NZV1

82-029362

6644

HD 183

HMC 46

HMC 56

$35000 \mathrm{HP}$

NZS1

NZS2

NZS3

NZS4

NZV1

$82-029362$

6644

HD 183

HMC 46

HMC56

$35000 \mathrm{HP}$
EPSSETPKSRGKRGISEESLSSTVQPRSRKISEEDSSFERLPLRI IDNGSDYAEILPRNV EPSSETPKSRGKRGISEESLSSTVOPRSRKISEEDSSFERLPLRIIDNGSDYAEILPRNV EPSSETPKSRGKRGISEESLSSTVQPRSRKISEEDSSFERLPLRIIDNGSDYAEILPRNV EPSSETPKSRGKRGISEESLSSTVQPRSRKISEEDSSFERLPLRI IDNGSDYAEILPRNV EPSSETPKSRGKRGISEESLSSTVQPRSRKISEEDSSFERLPLRIIDNGSDYAEILPRNV $* * * * * * * * * * * * * * * * * * * * * * * * * * * * * * * * * * * * * * * * * * * * * * * * * * * * * * * * * * * *$

KQTNEPATQAIRAPKALDNNDVIAERPSFKLRQLDDDVESVNGIYSS IKPKALIEEGTPI KQTNEPATQAIRAPKALDNNDVIAERP SFKLRQLDDDVESVNGIYSS IKPKALIEEGTPI KQTNEPATQAIRAPKALDNNDVIAERP SFKLRQLDDDVESVNGIYSS IKPKALIEEGTPI KOTNEPATOAIRAPKALDNNDVIAERPSFKLROLDDDVESVNGIYSS IKPKALIEEGTPI KQTNEPATQAIRAPKALDNNDVIAERP SFKLRQLDDDVESVNGIYSS IKPKALIEEGTPI KQTNEPATQAIRAPKALDNNDVIAERPSFKLRQLDDDVESVNGIYSS IKPKALIEEGTPI KQTNEPATQAIRAPKALDNNDVIAERP SFKLRQLDDDVESVNGIYSS IKPKALIEEGTPI KQTNEPATQAIRAPKALDNNDVIAERP SFKLRQLDDDVESVNG IYSS IKPKALIEEGTPI KOTNEPATOAIRAPKALDNNDVIAERPSFKLRQLDDDVESVNGIYSSIKPKALIEEGTPI KQTNEPATQA IRAPKALDNNDVIAERP SFKLRQLDDDVESVNGIYSS IKPKALIEEGTPI KQTNEPATQAIRAPKALDNNDVIAERPSFKLRQLDDDVESVNGIYSSIKPKALIEEGTPI $\star * * * * * * * * * * * * * * * * * * * * * * * * * * * * * * * * * * * * * * * * * * * * * * * * * * * * * * * * * * * * * * *$ t

TROVKTVOEETPVTDLVNKRELVKEDRSLLDKVODTFOPLKVRSKINDVRSSVEEYGGEV TRQVKTVQEETPVTNLVNKRELVKEDRSLLDKVQDTFQPLKVRSKINDVRSSVEEYGGEV TRQVKTVQEETPVTNLVNKRELVKEDRSLLDKVQDTFOPLKVRSKINDVRSSVEEYGGEV TRQVKTVQEETPVTNLVNKRELVKEDRSLLDKVQDTFQPLKVRSKINDVRSSVEEYGGEV TRQVKTVQEETPVTNLVNKRELVKEDRSLLDKVQDTFQPLKVRSKINDVRSSVEEYGGEV TRQVKTVQEETPVTNLVNKRELVKEDRSLLDKVQDTFOPLKVRSKINDVRSSVEEYGGEV TRQVKTVQEETPVTDLVNKRELVKEDRSLLDKVQDTFQPLKVRSKINDVRSSVEEYGGEV TROVKTVOEETPVTDLVNKRELVKEDRSLLDKVODTFOPLKVRSKINDVRSSVEEYGGEV TRQVKTVQEETPVTDLVNKRELVKEDRSLLDKVQDTFQPLKVRSKINDVRSSVEEYGGEV TRQVKTVQEETPVTDLVNKRELVKEDRSLLDKVQDTFQPLKVRSKINDVRSSVEEYGGEV TROVKTVQEETPVTDLVNKRELVKEDRSLLDKVQDTFOPLKVRSKINDVRSSVEEYGGEV $* * * * * * * * * * * * * *: * * * * * * * * * * * * * * * * * * * * * * * * * * * * * * * * * * * * * * * * * * * * *$

TFKYAQSKGEVYNEIVKHAETQNGVCEATCSHWIAKKVNDENIWTDLYKDGQKGRKGGLN TFKYAQSKGEVYNEIVKHAETQNGVCEATCSHWIAKKVNDEN IWTDLYKDGQKGRKGGLN TFKYAQSKGEVYNEIVKHAETQNGVCEATCSHWIAKKVNDENIWTDLYKDGQKGRKGGLN TFKYAQSKGEVYNEIVKHAETQNGVCEATCSHWIAKKVNDEN IWTDLYKDGQKGRKGGLN TFKYAQSKGEVYNEIVKHAETQNGVCEATCSHWIAKKVNDEN IWTDLYKDGQKGRKGGLN TFKYAQSKGEVYNEIVKHAETQNGVCEATCSHWIAKKVNDEN IWTDLYKDGQKGRKGGLN TFKYAOSKGEVYNEIVKHAETONGVCEATCSHWIAKKVNDEN IWTDLYKDGOKGRKGGLN TFKYAQSKGEVYNEIVKHAETQNGVCEATCSHWIAKKVNDEN IWTDLYKDGQKGRKGGLN TFKYAQSKGEVYNEIVKHAETQNGVCEATCSHWIAKKVNDENIWTDLYKDGQKGRKGGLN TFKYAQSKGEVYNEIVKHAETONGVCEATCSHWIAKKVNDENIWTDLYKDGOKGRKGGLN TFKYAQSKGEVYNEIVKHAETQNGVCEATCSHWIAKKVNDENIWTDLYKDGQKGRKGGLN

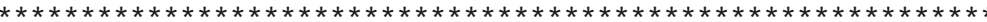

KDAIES IEKLQTEF INAGTATQOFKLTNTWLEEOGVVPKOKYFGKLSRADEVAGTVSKND KDAIES IEKLQTEF INAGTATQQFKLTNTWLEEQGVVPKQKYFGKLSRADEVAGTVSKND KDAIESIEKLQTEF INAGTATQQFKLTNTWLEEQGVVPKQKYFGKLSRADEVAGTVSKND KDAIES IEKLQTEF INAGTATQQFKLTNTWLEEQGVVPKQKYFGKLSRADEVAGTVSKND KDAIES IEKLQTEF INAGTATQQFKLTNTWLEEQGVVPKQKYFGKLSRADEVAGTVSKND KDAIES IEKLQTEF INAGTATQOFKLTNTWLEEQGVVPKOKYFGKLSRADEVAGTVSKND KDAIES IEKLQTEF INAGTATQQFKLTNTWLEEQGVVPKQKYFGKLSRADEVAGTVSKND KDAIES IEKLQTEF INAGTATQQFKLTNTWLEEQGVVPKQKYFGKLSRADEVAGTVSKND KDAIES IEKLQTEF INAGTATQQFKLTNTWLEEQGVVPKOKYFGKLSRADEVAGTVSKND KDAIES IEKLQTEF INAGTATQQFKLTNTWLEEQGVVPKOKYFGKLSRADEVAGTVSKND KDAIES IEKLQTEF INAGTATQQFKLTNTWLEEQGVVPKQKYFGKLSRADEVAGTVSKND $* * * * * * * * * * * * * * * * * * * * * * * * * * * * * * * * * * * * * * * * * * * * * * * * * * * * * * * * * * * *$

VSALVKAILDTGNESSAVKKIS INLEGGSHTVSASIEGQKVVFFDPNFGEITFKDKKSFE VSALVKAILDTGNESSAVKKIS INLEGGSHTVSASIEGQKVVFFDPNFGEITFKDKKSFE VSALVKAILDTGNESSAVKKISINLEGGSHTVSASIEGQKVVFFDPNFGEITFKDKKSFE VSALVKAILDTGNESSAVKKISINLEGGSHTVSASIEGQKVVFFDPNFGEITFKDKKSFE VSALVKAILDTGNESSAVKKIS INLEGGSHTVSASIEGQKVVFFDPNFGEITFKDKKSFE VSALVKAILDTGNESSAVKKIS INLEGGSHTVSASIEGQKVVFFDPNFGEITFKDKKSFE VSALVKAILDTGNESSAVKKISINLEGGSHTVSASIEGQKVVFFDPNFGEITFKDKKSFE VSALVKAILDTGNESSAVKKIS INLEGGSHTVSASIEGQKVVFFDPNFGEITFKDKKSFE VSALVKAILDTGNESSAVKKIS INLEGGSHTVSASIEGQKVVFFDPNFGEITFKDKKSFE VSALVKAILDTGNESSAVKKIS INLEGGSHTVSASIEGOKVVFFDPNFGEITFKDKKSFE VSALVKAILDTGNESSAVKKIS INLEGGSHTVSAS IEGQKVVFFDPNFGEITFKDKKSFE $* * * * * * * * * * * * * * * * * * * * * * * * * * * * * * * * * * * * * * * * * * * * * * * * * * * * * * * * * * * *$

KWMKNAFWKKSGYAGKKDTKRFFNVVNYHKNSKRNKVIDVNQNH IQQLAGSEGFSPSLPT 
NZS1

NZS2

NZS 3

NZS 4

NZV1

82-029362

6644

HD 183

HMC 46

HMC 56

$35000 \mathrm{HP}$

NZS 1

NZS2

NZS3

NZS4

NZV1

82-029362

6644

HD 183

HMC 46

HMC 56

$35000 \mathrm{HP}$

NZS 1

NZS2

NZS 3

NZS4

NZV1

82-029362

6644

HD 183

HMC 46

HMC 56

$35000 \mathrm{HP}$

NZS 1

NZS2

NZS 3

NZS 4

NZV1

82-029362

6644

HD 183

HMC 46

HMC 56

$35000 \mathrm{HP}$

NZS1

NZS2

NZS3

NZS4

NZV1

82-029362

6644

HD 183

HMC 46

HMC 56

$35000 \mathrm{HP}$

NZS 1

NZS2

NZS 3

NZS 4

NZV1

82-029362

6644

HD 183
KWMKNAFWKKSGYAGKKDTKRFFNVVNYHKNSKRNKVIDVNQNHIQQLAGSEGFSPSLPT KWMKNAFWKKSGYAGKKDTKRFFNVVNYHKNSKRNKVIDVNQNH IQQLAGSEGFSPSLPT KWMKNAFWKKSGYAGKKDTKRFFNVVNYHKNSKRNKVIDVNQNH IQQLAGSEGFSPSLPT KWMKNAFWKKSGYAGKKDTKRFFNVVNYHKNSKRNKVIDVNQNHIQQLAGSEGFSPSLPT KWMKNAFWKKSGYAGKKDTKRFFNVVNYHKNSKRNKVIDVNQNH IQQLAGSEGFSPSLPT KWMKNAFWKKSGYAGKKDTKRFFNVVNYHKNSKRNKVIDVNQNH IQQLAGSEGFSPSLPT KWMKNAFWKKSGYAGKKDTKRFFNVVNYHKNSKRNKVIDVNONH IOQLAGSEGFSPSLPT KWMKNAFWKKSGYAGKKDTKRFFNVVNYHKNSKRNKVIDVNQNHIQQLAGSEGFSPSLPT KWMKNAFWKKSGYAGKKDTKRFFNVVNYHKNSKRNKVIDVNQNHIQQLAGSEGFSPSLPT KWMKNAFWKKSGYAGKKDTKRFFNVVNYHKNSKRNKVIDVNQNHIQQLAGSEGFSPSLPT $* * * * * * * * * * * * * * * * * * * * * * * * * * * * * * * * * * * * * * * * * * * * * * * * * * * * * * * * * * * *$

RPQLANAAGIKSNEMSSLFSWSKLKHLFSRESGKKAQVEGPEIKHLGGVVDKDAFYFPLD RPOLANAAGIKSNEMSSLF SWSKLKHLFSRESGKKAOVEGPEIKHLGGVVDKDAFYFPLD RPQLANAAGIKSNEMSSLFSWSKLKHLFSRESGKKAQVEGPEIKHLGGVVDKDAFYFPLD RPQLANAAGIKSNEMSSLFSWSKLKHLFSRESGKKAQVEGPEIKHLGGVVDKDAFYFPLD RPQLANAAGIKSNEMSSLFSWSKLKHLF SRESGKKAQVEGPEIKHLGGVVDKDAFYFPLD RPQLANAAGIKSNEMSSLFSWSKLKHLFSRESGKKAQVEGPEIKHLGGVVDKDAFYFPLD RPQLANAAGIKSNEMSSLFSWSKLKHLF SRESGKKAQVEGPEIKHLGGVVDKDAFYFPLD RPQLANAAGIKSNEMSSLFSWSKLKHLFSRESGKKAQVEGPEIKHLGGVVDKDAFYFPLD RPQLANAAGIKSNEMSSLFSWSKLKHLF SRESGKKAQVEGPEIKHLGGVVDKDAFYFPLD RPQLANAAGIKSNEMSSLFSWSKLKHLFSRESGKKAQVEGPEIKHLGGVVDKDAFYFPLD RPQLANAAGIKSNEMSSLFSWSKLKHLFSRESGKKAQVEGPEIKHLGGVVDKDAFYFPLD $* * * * * * * * * * * * * * * * * * * * * * * * * * * * * * * * * * * * * * * * * * * * * * * * * * * * * * * * * * * *$

KIVTRRDAEGE IRVNMDNIKKAFNPRDKHYNSQEARSLRSLYNQDPSMSGTRF I IENQVI KIVTRRDAEGEIRVNMDN I KKAFNPRDKHYNSQEARSLRSLYNQDPSMSGTRF I IENQVI KIVTRRDAEGEIRVNMDNIKKAFNPRDKHYNSOEARSLRSLYNODPSMSGTRF I IENOVI KIVTRRDAEGEIRVNMDNI KKAFNPRDKHYNSQEARSLRSLYNQDPSMSGTRF I IENQVI KIVTRRDAEGEIRVNMDN I KKAFNPRDKHYNSQEARSLRSLYNQDPSMSGTRF I IENQVI KIVTRRDAEGE IRVNMDN I KKAFNPRDKHYNSQEARSLRSLYNQDP SMSGTRF I IENQVI KIVTRRDAEGEIRVNMDNI KKAFNPRDKHYNSQEARSLRSLYNQDPSMSGTRF I IENQVI KIVTRRDAEGE IRVNMDN I KKAFNPRDKHYNSQEARSLRSLYNQDPSMSGTRF I IENOVI KIVTRRDAEGE IRVNMDN I KKAFNPRDKHYNSQEARSLRSLYNQDPSMSGTRF I IENQVI KIVTRRDAEGEIRVNMDNIKKAFNPRDKHYNSQEARSLRSLYNQDPSMSGTRF I IENQVI KIVTRRDAEGEIRVNMDNIKKAFNPRDKHYNSQEARSLRSLYNQDPSMSGTRF I IENQVI $* * * * * * * * * * * * * * * * * * * * * * * * * * * * * * * * * * * * * * * * * * * * * * * * * * * * * * * * * * * *$

ANPFSSADLQSYIQAQQSKLPELGRQARRALPELPTAANKGRGSRVEEQNIVTRPRVEDV ANPFSSADLOSYIOAOQSKLPELGROARRALPELPTAANKGRGSRVEEONIVTRPRVEDV ANPFSSADLQSYIQAQQSKLPELGRQARRALPELPTAANKGRGSRVEEQNIVTRPRVEDV ANPFSSADLQSYIQAQQSKLPELGRQARRALPELPTAANKGRGSRVEEQNIVTRPRVEDV ANPFSSADLOSYIOAOOSKLPELGROARRALPELPTAANKGRGSRVEEONIVTRPRVEDV ANPFSSADLQSYIQAQQSKLPELGRQARRALPELPTAANKGRGSRVEEQNIVTRPRVEDV ANPFSSADLQSYIQAQQSKLPELGRQARRALPELPTAANKGRGSRVEEQNIVTRPRVEDV ANPFSSADLQSYIQAQQSKLPELGRQARRALPELPTAANKGRGSRVEEQN IVTRPRVEDV ANPFSSADLQSYIQAQQSKLPELGRQARRALPELPTAANKGRGSRVEEQNIVTRPRVEDV ANPFSSADLQSYIQAQQSKLPELGRQARRALPELPTAANKGRGSRVEEQNIVTRPRVEDV ANPFSSADLQSYIQAQQSKLPELGRQARRALPELPTAANKGRGSRVEEQNIVTRPRVEDV $* * * * * * * * * * * * * * * * * * * * * * * * * * * * * * * * * * * * * * * * * * * * * * * * * * * * * * * * * * * *$

YATVNKGAKHGEAQQPGSFYTKKLVDQVSHVPNTEPVYADLHFNRNGRVVRQTEPEVIYE YATVNKGAKHGEAQQPGSFYTKKLVDQVSHVPNTEPVYADLHFNRNGRVVRQTEPEVIYE YATVNKGAKHGEAQQPGSFYTKKLVDQVSHVPNTEPVYADLHFNRNGRVVRQTEPEVIYE YATVNKGAKHGEAQQPGSFYTKKLVDQVSHVPNTEPVYADLHFNRNGRVVRQTEPEVIYE YATVNKGAKHGEAQQPGSFYTKKLVDQVSHVPNTEPVYADLHFNRNGRVVRQTEPEVIYE YATVNKGAKHGEAQQPGSFYTKKLVDQVSHVPNTEPVYADLHFNRNGRVVRQTEPEVIYE YATVNKGAKHGEAQQPGSFYTKKLVDQVSHVPTTEPVYADLHFNRNGRVVRQTEPEVIYE YATVNKGAKHGEAQQPGSFYTKKLVDQVSHVPTTEPVYADLHFNRNGRVVRQTEPEVIYE YATVNKGAKHGEAQQPGSFYTKKLVDQVSHVPNTEPVYADLHFNRNGRVVRQTEPEVIYE YATVNKGAKHGEAQQPGSFYTKKLVDQVSHVPTTEPVYADLHFNRNGRVVRQTEPEVIYE YATVNKGAKHGEAQQPGSFYTKKLVDQVSHVPTTEPVYADLHFNRNGRVVRQTEPEVIYE $* * * * * * * * * * * * * * * * * * * * * * * * * * * * * * * * . * * * * * * * * * * * * * * * * * * * * * * * * * * *$

KIRGQQVEVDDPSSLYAKVNRNRRLDNVEGFYPPEQLRTRSDKLAEQVSRVPTTEPVYAD KIRGQQVEVDDPSSLYAKVNRNRRLDNVEGFYPPEQLRTRSDKLAEQVSRVPTTEPVYAD KIRGQQVEVDDPSSLYAKVNRNRRLDNVEGFYPPEQLRTRSDKLAEQVSRVPTTEPVYAD KIRGQQVEVDDPSSLYAKVNRNRRLDNVEGFYPPEQLRTRSDKLAEQVSRVPTTEPVYAD KIRGQQVEVDDPSSLYAKVNRNRRLDNVEGFYPPEQLRTRSDKLAEQVSRVPTTEPVYAD KIRGQQVEVDDPSSLYAKVNRNRRLDNVEGFYPPEQLRTRSDKLAEQVSRVPTTEPVYAD KIRGQQVEVDDPSSLYAKVNRNRRLDNVEGFYPPEQLRTRSDKLAEQVSRVPTTEPVYAD KIRGQQVEVDDPSSLYAKVNRNRRLDNVEGFYPPEQLRTRSDKLAEQVSRVPTTEPVYAD KIRGQQVEVDDPSSLYAKVNRNRRLDNVEGFYPPEQLRTRSDKLAEQVSRVPTTEPVYAD 
HMC 46

HMC 56

$35000 \mathrm{HP}$

NZS 1

NZS2

NZS 3

NZS 4

NZV1

82-029362

6644

HD 183

HMC 46

HMC 56

$35000 \mathrm{HP}$

NZS1

NZS2

NZS 3

NZS4

NZV1

82-029362

6644

HD 183

HMC 46

HMC 56

$35000 \mathrm{HP}$

NZS1

NZS2

NZS 3

NZS 4

NZV1

82-029362

6644

HD 183

HMC 46

HMC 56

$35000 \mathrm{HP}$

NZS 1

NZS2

NZS3

NZS4

NZV1

82-029362

6644

HD 183

HMC 46

HMC 56

$35000 \mathrm{HP}$

NZS 1

NZS2

NZS3

NZS4

NZV1

$82-029362$

6644

HD183

HMC 46

HMC5 6

$35000 \mathrm{HP}$

NZS1

NZS2

NZS3
KIRGQQVEVDDPSSLYAKVNRNRRLDNVEGFYPPEQLRTRSDKLAEQVSRVPTTEPVYAD KIRGQQVEVDDPSSLYAKVNRNRRLDNVEGFYPPEQLRTRSDKLAEQVSRVPTTEPVYAD $* * * * * * * * * * * * * * * * * * * * * * * * * * * * * * * * * * * * * * * * * * * * * * * * * * * * * * * * * * * *$

LRFKSAEDDYAPALPARPELGNAAGFRKAKVKGEESESTWSRLKHLFSRESGKKAQVEGP LRFKSAEDDYAPALPARPELGNAAGFRKAKVKGEESESTWSRLKHLF SRESGKKAQVEGP LRFKSAEDDYAPALPARPELGNAAGFRKAKVKGEESESTWSRLKHLF SRESGKKAQVEGP LRFKSAEDDYAPALPARPELGNAAGFRKAKVKGEESESTWSRLKHLF SRESGKKAQVEGP LRFKSAEDDYAPALPARPELGNAAGFRKAKVKGEESESTWSRLKHLFSRESGKKAQVEGP LRFKSAEDDYAPALPARPELGNAAGFRKAKVKGEESESTWSRLKHLF SRESGKKAQVEGP LRFKSAEDDYAPALPARPELGNAAGFRKAKVKGEESESTWSRLKHLF SRESGKKAOVEGP LRFKSAEDDYAPALPARPELGNAAGFRKAKVKGEESESTWSRLKHLFSRESGKKAQVEGP LRFKSAEDDYAPALPARPELGNAAGFRKAKVKGEESESTWSRLKHLF SRESGKKAQVEGP LRFKSAEDDYAPALPARPELGNAAGFRKAKVKGEESESTWSRLKHLF SRESGKKAQVEGP LRFKSAEDDYAPALPARPELGNAAGFRKAKVKGEESESTWSRLKHLFSRESGKKAQVEGP $* * * * * * * * * * * * * * * * * * * * * * * * * * * * * * * * * * * * * * * * * * * * * * * * * * * * * * * * * * * * *$

EIKHLGGVVDKDAFYFPLDKIVTRRDAEGEIRVNMDNIKKAFNPRDKHYNSOEARSLRSL EIKHLGGVVDKDAFYFPLDKIVTRRDAEGE IRVNMDNI KKAFNPRDKHYNSQEARSLRSI EIKHLGGVVDKDAFYFPLDKIVTRRDAEGEIRVNMDNIKKAFNPRDKHYNSQEARSLRSL EIKHLGGVVDKDAFYFPLDKIVTRRDAEGEIRVNMDNIKKAFNPRDKHYNSQEARSLRSL EIKHLGGVVDKDAFYFPLDKIVTRRDAEGEIRVNMDNIKKAFNPRDKHYNSOEARSLRSL EIKHLGGVVDKDAFYFPLDKIVTRRDAEGEIRVNMDNIKKAFNPRDKHYNSQEARSLRSL EIKHLGGVVDKDAFYFPLDKIVTRRDAEGE IRVNMDNIKKAFNPRDKHYNSQEARSLRSL EIKHLGGVVDKDAFYFPLDKIVTRRDAEGE IRVNMDNIKKAFNPRDKHYNSOEARSLRSL EIKHLGGVVDKDAFYFPLDKIVTRRDAEGEIRVNMDNIKKAFNPRDKHYNSQEARSLRSL EIKHLGGVVDKDAFYFPLDKIVTRRDAEGEIRVNMDNI KKAFNPRDKHYNSQEARSLRSL EIKHLGGVVDKDAFYFPLDKIVTRRDAEGE IRVNMDNIKKAFNPRDKHYNSQEARSLRSL $* * * * * * * * * * * * * * * * * * * * * * * * * * * * * * * * * * * * * * * * * * * * * * * * * * * * * * * * * * * * * *$

YNQDPSMSGTRF I IENOVIANPF SSADLQSYIOAOQSKLPELGROARRALPELPTAANKG YNQDPSMSGTRF I IENQVIANPFSSADLQSYIQAQQSKLPELGRQARRALPELPTAANKG YNQDPSMSGTRF I IENQVIANPF SSADLQSY IQAQQSKLPELGRQARRALPELPTAANKG YNQDPSMSGTRF I IENQVIANPFSSADLQSYIQAQQSKLPELGRQARRALPELPTAANKG YNQDPSMSGTRF I IENQVIANPF SSADLQSY IQAQQSKLPELGRQARRALPELPTAANKG YNQDPSMSGTRF I IENQVIANPFSSADLQSYIQAQQSKLPELGRQARRALPELPTAANKG YNQDPSMSGTRF I IENQVIANPF SSADLQSYIQAQQSKLPELGRQARRALPELPTAANKG YNQDPSMSGTRF I IENQVIANPFSSADLQSYIQAQQSKLPELGRQARRALPELPTAANKG YNQDPSMSGTRF I IENQVIANPFSSADLQSYIQAQQSKLPELGRQARRALPELPTAANKG YNODPSMSGTRF I IENOVIANPFSSADLOSYIOAOOSKLPELGROARRALPELPTAANKG YNQDPSMSGTRF I IENQVIANPFSSADLQSYIQAQQSKLPELGRQARRALPELPTAANKG $* * * * * * * * * * * * * * * * * * * * * * * * * * * * * * * * * * * * * * * * * * * * * * * * * * * * * * * * * * * * * *$

RGSRVEEQNIVTRPRVEDVYATVNKGAKHGEAQQPGSFYTKKLVDQVSHVPNTEPVYADL RGSRVEEONIVTRPRVEDVYATVNKGAKHGEAOOPGSFYTKKLVDOVSHVPNTEPVYADL RGSRVEEQNIVTRPRVEDVYATVNKGAKHGEAQQPGSFYTKKLVDQVSHVPNTEPVYADL RGSRVEEQNIVTRPRVEDVYATVNKGAKHGEAQQPGSFYTKKLVDQVSHVPNTEPVYADL RGSRVEEQNIVTRPRVEDVYATVNKGAKHGEAQQPGSFYTKKLVDQVSHVPNTEPVYADL RGSRVEEQNIVTRPRVEDVYATVNKGAKHGEAQQPGSFYTKKLVDQVSHVPNTEPVYADL RGSRVEEQNIVTRPRVEDVYATVNKGAKHGEAQQPGSFYTKKLVDQVSHVPTTEPVYADL RGSRVEEQNIVTRPRVEDVYATVNKGAKHGEAQQPGSFYTKKLVDQVSHVPTTEPVYADL RGSRVEEQNIVTRPRVEDVYATVNKGAKHGEAQQPGSFYTKKLVDQVSHVPNTEPVYADL RGSRVEEQNIVTRPRVEDVYATVNKGAKHGEAQQPGSFYTKKLVDQVSHVPTTEPVYADL RGSRVEEQNIVTRPRVEDVYATVNKGAKHGEAQQPGSFYTKKLVDQVSHVPTTEPVYADL $\star * * * * * * * * * * * * * * * * * * * * * * * * * * * * * * * * * * * * * * * * * * * * * * * * * * . * * * * * * * *$

HFNRNGRVVRQTEPEVIYEKIRGQQVEVDDPSSLYAKVNRNRRLDNVEGFYPPEQLRTRS HFNRNGRVVRQTEPEVIYEKIRGQQVEVDDPSSLYAKVNRNRRLDNVEGFYPPEQLRTRS HFNRNGRVVRQTEPEVIYEKIRGQQVEVDDPSSLYAKVNRNRRLDNVEGFYPPEQLRTRS HFNRNGRVVROTEPEVIYEKIRGQOVEVDDPSSLYAKVNRNRRLDNVEGFYPPEQLRTRS HFNRNGRVVRQTEPEVIYEKIRGQQVEVDDPSSLYAKVNRNRRLDNVEGFYPPEQLRTRS HFNRNGRVVRQTEPEVIYEKIRGQQVEVDDPSSLYAKVNRNRRLDNVEGFYPPEQLRTRS HFNRNGRVVRQTEPEVIYEKIRGQQVEVDDPSSLYAKVNRNRRLDNVEGFYPPEQLRTRS HFNRNGRVVRQTEPEVIYEKIRGQQVEVDDPSSLYAKVNRNRRLDNVEGFYPPEQLRTRS HFNRNGRVVRQTEPEVIYEKIRGQQVEVDDPSSLYAKVNRNRRLDNVEGFYPPEQLRTRS HFNRNGRVVRQTEPEVIYEKIRGQQVEVDDPSSLYAKVNRNRRLDNVEGFYPPEQLRTRS HFNRNGRVVRQTEPEVIYEKIRGQQVEVDDPSSLYAKVNRNRRLDNVEGFYPPEQLRTRS $* * * * * * * * * * * * * * * * * * * * * * * * * * * * * * * * * * * * * * * * * * * * * * * * * * * * * * * * * * * *$

DKLAEQVSRVPTTEPVYADLRFKSAEDDYAPALPARPELGNAAGFRKAKVKGEESESTWS DKLAEQVSRVPTTEPVYADLRFKSAEDDYAPALPARPELGNAAGFRKAKVKGEESESTWS DKLAEQVSRVPTTEPVYADLRFKSAEDDYAPALPARPELGNAAGFRKAKVKGEESESTWS DKLAEQVSRVPTTEPVYADLRFKSAEDDYAPALPARPELGNAAGFRKAKVKGEESESTWS 
NZS4

NZV1

82-029362

6644

HD 183

HMC 46

HMC 56

$35000 \mathrm{HP}$

NZS 1

NZS2

NZS 3

NZS4

NZV1

82-029362

6644

HD 183

HMC 46

HMC 56

$35000 \mathrm{HP}$

NZS 1

NZS2

NZS3

NZS4

NZV1

82-029362

6644

HD 183

HMC 46

HMC 56

$35000 \mathrm{HP}$

NZS1

NZS2

NZS 3

NZS 4

NZV1

82-029362

6644

HD 183

HMC 46

HMC 56

$35000 \mathrm{HP}$

NZS 1

NZS2

NZS3

NZS 4

NZV1

82-029362

6644

HD 183

HMC 46

HMC 56

$35000 \mathrm{HP}$

NZS 1

NZS2

NZS3

NZS4

NZV1

$82-029362$

6644

HD 183

HMC 46

HMC 56
DKLAEQVSRVPTTEPVYADLRFKSAEDDYAPALPARPELGNAAGFRKAKVKGEESESTWS DKLAEQVSRVPTTEPVYADLRFKSAEDDYAPALPARPELGNAAGFRKAKVKGEESESTWS DKLAEQVSRVPTTEPVYADLRFKSAEDDYAPALPARPELGNAAGFRKAKVKGEESESTWS DKLAEOVSRVPTTEPVYADLRFKSAEDDYAPALPARPELGNAAGFRKAKVKGEESESTWS DKLAEQVSRVPTTEPVYADLRFKSAEDDYAPALPARPELGNAAGFRKAKVKGEESESTWS DKLAEQVSRVPTTEPVYADLRFKSAEDDYAPALPARPELGNAAGFRKAKVKGEESESTWS DKLAEQVSRVPTTEPVYADLRFKSAEDDYAPALPARPELGNAAGFRKAKVKGEESESTWS $* * * * * * * * * * * * * * * * * * * * * * * * * * * * * * * * * * * * * * * * * * * * * * * * * * * * * * * * * * * *$

RLKHLFSRESGKKAQVEGPEIKHLGGVVDKDAFYFPLDKIVTRRDAEGE IRVNMDNIKKA RLKHLFSRESGKKAOVEGPEI KHLGGVVDKDAFYFPLDKIVTRRDAEGE IRVNMDNIKKA RLKHLFSRESGKKAQVEGPEIKHLGGVVDKDAFYFPLDKIVTRRDAEGE IRVNMDNIKKA RLKHLFSRESGKKAQVEGPEIKHLGGVVDKDAFYFPLDKIVTRRDAEGEIRVNMDNIKKA RLKHLFSRESGKKAQVEGPEIKHLGGVVDKDAFYFPLDKIVTRRDAEGE IRVNMDNIKKA RLKHLFSRESGKKAQVEGPEIKHLGGVVDKDAFYFPLDKIVTRRDAEGE IRVNMDNIKKA RLKHLFSRESGKKAQVEGPEIKHLGGVVDKDAFYFPLDKIVTRRDAEGE IRVNMDNIKKA RLKHLFSRESGKKAQVEGPEIKHLGGVVDKDAFYF PLDKIVTRRDAEGE IRVNMDNIKKA RLKHLFSRESGKKAQVEGPEIKHLGGVVDKDAFYFPLDKIVTRRDAEGEIRVNMDNIKKA RLKHLFSRESGKKAQVEGPEIKHLGGVVDKDAFYFPLDKIVTRRDAEGE IRVNMDNIKKA RLKHLFSRESGKKAQVEGPEIKHLGGVVDKDAFYFPLDKIVTRRDAEGE IRVNMDNIKKA $* * * * * * * * * * * * * * * * * * * * * * * * * * * * * * * * * * * * * * * * * * * * * * * * * * * * * * * * * * * *$

FNPRDKHYNSQEARSLRSLYNQDPSMSGTRF I IENQVIANPFSSADLQSYIQAQQSKLPE FNPRDKHYNSQEARSLRSLYNQDPSMSGTRF I IENQVIANPF SSADLQSYIQAQQSKLPE FNPRDKHYNSQEARSLRSLYNQDPSMSGTRF I IENQVIANPFSSADLQSYIQAQQSKLPE FNPRDKHYNSQEARSLRSLYNQDPSMSGTRF I IENQVIANPF SSADLQSYIQAQQSKLPE FNPRDKHYNSQEARSLRSLYNQDPSMSGTRF I IENQVIANPF SSADLQSYIQAQQSKLPE FNPRDKHYNSOEARSLRSLYNQDPSMSGTRF I IENQVIANPFSSADLQSY IQAQQSKLPE FNPRDKHYNSQEARSLRSLYNQDPSMSGTRF I IENQVIANPF SSADLQSY IQAQQSKLPE FNPRDKHYNSQEARSLRSLYNQDPSMSGTRF I IENQVIANPFSSADLQSYIQAQQSKLPE FNPRDKHYNSQEARSLRSLYNQDPSMSGTRF I IENQVIANPF SSADLQSY IQAQQSKLPE FNPRDKHYNSQEARSLRSLYNQDPSMSGTRF I IENQVIANPF SSADLQSYIQAQQSKLPE FNPRDKHYNSQEARSLRSLYNQDPSMSGTRF I IENQVIANPFSSADLQSYIQAQQSKLPE $* * * * * * * * * * * * * * * * * * * * * * * * * * * * * * * * * * * * * * * * * * * * * * * * * * * * * * * * * * * *$

LGRQARRALPELPTAANKGRGSRVEEQNIVTRPRVEDVYATVNKGAKHGEAQQPGSFYTK LGRQARRALPELPTAANKGRGSRVEEQNIVTRPRVEDVYATVNKGAKHGEAQQPGSFYTK LGRQARRALPELPTAANKGRGSRVEEQNIVTRPRVEDVYATVNKGAKHGEAQQPGSFYTK LGRQARRALPELPTAANKGRGSRVEEQNIVTRPRVEDVYATVNKGAKHGEAQQPGSFYTK LGROARRALPELPTAANKGRGSRVEEQNIVTRPRVEDVYATVNKGAKHGEAQQPGSFYTK LGRQARRALPELPTAANKGRGSRVEEQNIVTRPRVEDVYATVNKGAKHGEAQQPGSFYTK LGRQARRALPELPTAANKGRGSRVEEQNIVTRPRVEDVYATVNKGAKHGEAQQPGSFYTK LGRQARRALPELPTAANKGRGSRVEEQNIVTRPRVEDVYATVNKGAKHGEAQQPGSFYTK LGRQARRALPELPTAANKGRGSRVEEQNIVTRPRVEDVYATVNKGAKHGEAQQPGSFYTK LGRQARRALPELPTAANKGRGSRVEEQNIVTRPRVEDVYATVNKGAKHGEAQQPGSFYTK LGRQARRALPELPTAANKGRGSRVEEQNIVTRPRVEDVYATVNKGAKHGEAQQPGSFYTK $* * * * * * * * * * * * * * * * * * * * * * * * * * * * * * * * * * * * * * * * * * * * * * * * * * * * * * * * * * * *$

KLVDQVSHVPNTEPVYADLHFNRNGRVVRQTEPEVIYEKIRGQQVEVDDPSSLYAKVNRN KLVDQVSHVPNTEPVYADLHFNRNGRVVRQTEPEVIYEKIRGQQVEVDDP SSLYAKVNRN KLVDQVSHVPNTEPVYADLHFNRNGRVVRQTEPEVIYEKIRGQQVEVDDPSSLYAKVNRN KLVDQVSHVPNTEPVYADLHFNRNGRVVRQTEPEVIYEKIRGQQVEVDDPSSLYAKVNRN KLVDQVSHVPNTEPVYADLHFNRNGRVVRQTEPEVIYEKIRGQQVEVDDPSSLYAKVNRN KLVDQVSHVPNTEPVYADLHFNRNGRVVRQTEPEVIYEKIRGQQVEVDDPSSLYAKVNRN KLVDQVSHVPTTEPVYADLHFNRNGRVVRQTEPEVIYEKIRGQQVEVDDPSSLYAKVNRN KLVDQVSHVPTTEPVYADLHFNRNGRVVRQTEPEVIYEKIRGQQVEVDDPSSLYAKVNRN KLVDQVSHVPNTEPVYADLHFNRNGRVVRQTEPEVIYEKIRGQQVEVDDPSSLYAKVNRN KLVDQVSHVPTTEPVYADLHFNRNGRVVRQTEPEVIYEKIRGQQVEVDDPSSLYAKVNRN KLVDQVSHVPTTEPVYADLHFNRNGRVVRQTEPEVIYEKIRGQQVEVDDPSSLYAKVNRN $* * * * * * * * * * . * * * * * * * * * * * * * * * * * * * * * * * * * * * * * * * * * * * * * * * * * * * * * * * * *$

RRLDNVEGFYPPEQLRTRSDKLAEQVSRVPTTEPVYADLRFKSAEDDYAPALPARPELGN RRLDNVEGFYPPEQLRTRSDKLAEQVSRVPTTEPVYADLRFKSAEDDYAPALPARPELGN RRLDNVEGFYPPEQLRTRSDKLAEQVSRVPTTEPVYADLRFKSAEDDYAPALPARPELGN RRLDNVEGFYPPEQLRTRSDKLAEOVSRVPTTEPVYADLRFKSAEDDYAPALPARPELGN RRLDNVEGFYPPEQLRTRSDKLAEQVSRVPTTEPVYADLRFKSAEDDYAPALPARPELGN RRLDNVEGFYPPEQLRTRSDKLAEQVSRVPTTEPVYADLRFKSAEDDYAPALPARPELGN RRLDNVEGFYPPEQLRTRSDKLAEQVSRVPTTEPVYADLRFKSAEDDYAPALPARPELGN RRLDNVEGFYPPEQLRTRSDKLAEOVSRVPTTEPVYADLRFKSAEDDYAPALPARPELGN RRLDNVEGFYPPEQLRTRSDKLAEQVSRVPTTEPVYADLRFKSAEDDYAPALPARPELGN RRLDNVEGFYPPEQLRTRSDKLAEQVSRVPTTEPVYADLRFKSAEDDYAPALPARPELGN RRLDNVEGFYPPEQLRTRSDKLAEQVSRVPTTEPVYADLRFKSAEDDYAPALPARPELGN $* * * * * * * * * * * * * * * * * * * * * * * * * * * * * * * * * * * * * * * * * * * * * * * * * * * * * * * * * * * *$ 
$35000 \mathrm{HP}$

NZS 1

NZS2

NZS 3

NZS 4

NZV1

82-029362

6644

HD 183

HMC 46

HMC 56

$35000 \mathrm{HP}$

NZS 1

NZS2

NZS 3

NZS 4

NZV1

82-029362

6644

HD 183

HMC 46

HMC 56

$35000 \mathrm{HP}$

NZS 1

NZS2

NZS3

NZS4

NZV1

$82-029362$

6644

HD1 83

HMC 46

HMC 56

$35000 \mathrm{HP}$

NZS 1

NZS2

NZS 3

NZS 4

NZV1

82-029362

6644

HD 183

HMC 46

HMC 56

$35000 \mathrm{HP}$

NZS 1

NZS2

NZS 3

NZS 4

NZV1

82-029362

6644

HD 183

HMC 46

HMC 56

$35000 \mathrm{HP}$

NZS1

NZS2

NZS 3

NZS 4

NZV1

82-029362
AAGFRKAKVKGEESESTWSRLKHLFSRESGKTKVEEVENEYKSOTNGENSLEIKTIEHIP AAGFRKAKVKGEESESTWSRLKHLFSRESGKTKVEEVENEYKSQTNGENSLEIKTIEHIP AAGFRKAKVKGEESESTWSRLKHLF SRESGKTKVEEVENEYKSQTNGENSLE IKTIEH IP AAGFRKAKVKGEESESTWSRLKHLF SRESGKTKVEEVENEYKSQTNGENSLEIKTIEHIP AAGFRKAKVKGEESESTWSRLKHLFSRESGKTKVEEVENEYKSQTNGENSLEIKTIEHIP AAGFRKAKVKGEESESTWSRLKHLFSRESGKTKVEEVENEYKSOTNGENSLEIKTIEHIP AAGFRKAKVKGEESESTWSRLKHLFSRESGKTKVEEVENEYKSQTNGENSLEIKTIEHIP AAGFRKAKVKGEESESTWSRLKHLFSRESGKTKVEEVENEYKSOTNGENSLEIKTIEHIP AAGFRKAKVKGEESESTWSRLKHLF SRESGKTKVEEVENEYKSQTNGENSLEIKTIEHIP AAGFRKAKVKGEESESTWSRLKHLFSRESGKTKVEEVENEYKSOTNGENSLEIKTIEHIP AAGFRKAKVKGEESESTWSRLKHLF SRESGKTKVEEVENEYKSQTNGENSLEIKTIEHIP $\star * * * * * * * * * * * * * * * * * * * * * * * * * * * * * * * * * * * * * * * * * * * * * * * * * * * * * * * * * * * * * *$

TRLKNLESPREAQSDLGENALIYGLQRGRQALISKANAADKEGKNAILADSYIGKLNLGF TRLKNLESPREAQSDLGENALIYGLQRGRQALISKANAADKEGKNAILADSYIGKLNLGF TRLKNLESPREAQSDLGENALIYGLQRGRQALISKANAADKEGKNAILADSYIGKLNLGF TRLKNLESPREAQSDLGENALIYGLQRGRQALISKANAADKEGKNAILADSYIGKLNLGF TRLKNLESPREAQSDLGENALIYGLQRGRQALISKANAADKEGKNAILADSYIGKLNLGF TRLKNLESPREAQSDLGENALI YGLQRGRQALISKANAADKEGKNAILADSYIGKLNLGF TRLKNLESPREAQSDLGENAL IYGLQRGRQALISKANAADKEGKNAILADSYIGKLNLGF TRLKNLESPREAQSDLGENAL I YGLQRGRQALISKANAADKEGKNAILADSY IGKLNLGF TRLKNLESPREAQSDLGENAL IYGLQRGRQALISKANAADKEGKNAILADSYIGKLNLGF TRLKNLESPREAQSDLGENALIYGLQRGRQALISKANAADKEGKNAILADSY IGKLNLGF TRLKNLESPREAQSDLGENALIYGLQRGRQALISKANAADKEGKNAILADSYIGKLNLGF *************************************************************1

EFGELTKFAKOVKDGKVTEODIONIASFNDETAKLARRSEPKNRINDANVDDNORI IREL EFGELTKFAKQVKDGKVTEQDIQNIASFNDETAKLARRSEPKNRINDANVDDNQRI IREL EFGELTKFAKQVKDGKVTEQDIQNIASFNDETAKLARRSEPKNRINDANVDDNQRI IREL EFGELTKFAKOVKDGKVTEQDIONIASFNDETAKLARRSEPKNRINDANVDDNQRI IREL EFGELTKFAKQVKDGKVTEQDIQNIASFNDETAKLARRSEPKNRINDANVDDNQRIIREL EFGELTKFAKQVKDGKVTEQDIQNIASFNDETAKLARRSEPKNRINDANVDDNQRI IREL EFGELTKFAKOVKDGKVTEQDIONIASFNDETAKLARRSEPKNRINDANVDDNQRI IREL EFGELTKFAKQVKDGKVTEQDIQNIASFNDETAKLARRSEPKNRINDANVDDNQRI IREL EFGELTKFAKOVKDGKVTEODIONIASFNDETAKLARRSEPKNRINDANVDDNORI IREL EFGELTKFAKQVKDGKVTEQDIQNIASFNDETAKLARRSEPKNRINDANVDDNQRIIREL EFGELTKFAKOVKDGKVTEQDIONIASFNDETAKLARRSEPKNRINDANVDDNORI IREI $* * * * * * * * * * * * * * * * * * * * * * * * * * * * * * * * * * * * * * * * * * * * * * * * * * * * * * * * * * * *$

INNEAAVDALKRIATLSDQEKAMHSTLRANEKFDMDELEESPNYTTAENKS IRDYKDTQK INNEAAVDALKRIATLSDQEKAMHSTLRANEKFDMDELEESPNYTTAENKS IRDYKDTOK INNEAAVDALKRIATLSDOEKAMHSTLRANEKFDMDELEESPNYTTAENKS IRDYKDTOK INNEAAVDALKRIATLSDQEKAMHSTLRANEKFDMDELEESPNYTTAENKSIRDYKDTQK INNEAAVDALKRIATLSDOEKAMHSTLRANEKFDMDELEESPNYTTAENKS IRDYKDTOK INNEAAVDALKRIATLSDQEKAMHSTLRANEKFDMDELEESPNYTTAENKSIRDYKDTOK INNEAAVDALKRIATLSDQEKAMHSTLRANEKFDMDELEESPNYTTAENKS IRDYKDTQK INNEAAVDALKRIATLSDQEKAMHSTLRANEKFDMDELEESPNYTTAENKS IRDYKDTQK INNEAAVDALKRIATLSDQEKAMHSTLRANEKFDMDELEESPNYTTAENKS IRDYKDTQK INNEAAVDALKRIATLSDQEKAMHSTLRANEKFDMDELEESPNYTTAENKS IRDYKDTQK INNEAAVDALKRIATLSDQEKAMHSTLRANEKFDMDELEESPNYTTAENKS IRDYKDTQK $* * * * * * * * * * * * * * * * * * * * * * * * * * * * * * * * * * * * * * * * * * * * * * * * * * * * * * * * * * * *$

ALNDARMDFFTEKTKF IAKETLERGGQLYFALDGLVTNSPGFRADTQINMDKLKDVFNPN ALNDARMDFFTEKTKF IAKETLERGGQLYFALDGLVTNSPGFRADTQINMDKLKDVFNPN ALNDARMDFFTEKTKF IAKETLERGGQLYFALDGLVTNSPGFRADTQINMDKLKDVFNPN ALNDARMDFFTEKTKF IAKETLERGGOLYFALDGLVTNSPGFRADTOINMDKLKDVFNPN ALNDARMDFFTEKTKFIAKETLERGGQLYFALDGLVTNSPGFRADTQINMDKLKDVFNPN ALNDARMDFFTEKTKF IAKETLERGGQLYFALDGLVTNSPGFRADTQINMDKLKDVFNPN ALNDARMDFFTEKTKF IAKETLERGGOLYFALDGLVTNSPGFRADTOINMDKLKDVFNPN ALNDARMDFFTEKTKF IAKETLERGGQLYFALDGLVTNSPGFRADTQINMDKLKDVFNPN ALNDARMDFFTEKTKF IAKETLERGGOLYFALDGLVTNSPGFRADTOINMDKLKDVFNPN ALNDARMDFFTEKTKFIAKETLERGGQLYFALDGLVTNSPGFRADTQINMDKLKDVFNPN ALNDARMDFFTEKTKF IAKETLERGGQLYFALDGLVTNSPGFRADTQINMDKLKDVFNPN $* * * * * * * * * * * * * * * * * * * * * * * * * * * * * * * * * * * * * * * * * * * * * * * * * * * * * * * * * * * *$

HEHYDSVTSRELRYLYENYKDNPNLKFTLKDHVIANPLKTLKTS ISESDLKSSPRRARQE HEHYDSVTSRELRYLYENYKDNPNLKFTLKDHVIANPLKTLKTS ISESDLKSSPRRARQE HEHYDSVTSRELRYLYENYKDNPNLKFTLKDHVIANPLKTLKTS ISESDLKSSPRRAROE HEHYDSVTSRELRYLYENYKDNPNLKFTLKDHVIANPLKTLKTS ISESDLKSSPRRARQE HEHYDSVTSRELRYLYENYKDNPNLKFTLKDHVIANPLKTLKTS ISESDLKSSPRRAROE HEHYDSVTSRELRYLYENYKDNPNLKFTLKDHVIANPLKTLKTS ISESDLKSSPRRAROE HEHYDSVTSRELRYLYENYKDNPNLKFTLKDHVIANPLKTLKTSISESDLKSSPRRARQE 
6644

HD 183

HMC 46

HMC 56

$35000 \mathrm{HP}$

NZS 1

NZS2

NZS 3

NZS 4

NZV1

82-029362

6644

HD 183

HMC 46

HMC 56

$35000 \mathrm{HP}$

NZS 1

NZS2

NZS 3

NZS 4

NZV1

82-029362

6644

HD 183

HMC 46

HMC 56

$35000 \mathrm{HP}$

NZS 1

NZS2

NZS 3

NZS 4

NZV1

82-029362

6644

HD 183

HMC 46

HMC 56

$35000 \mathrm{HP}$

NZ S 1

NZS2

NZS 3

NZS 4

NZV1

82-029362

6644

HD 183

HMC 46

HMC 56

$35000 \mathrm{HP}$

NZS 1

NZS2

NZS 3

NZS4

NZV1

82-029362

6644

HD 183

HMC 46

HMC 56
HEHYDSVTSRELRYLYENYKDNPNLKFTLKDHVIANPLKTLKTS ISESDLKSSPRRAROE HEHYDSVTSRELRYLYENYKDNPNLKFTLKDHVIANPLKTLKTSISESDLKSSPRRARQE HEHYDSVTSRELRYLYENYKDNPNLKFTLKDHVIANPLKTLKT SISESDLKSSPRRARQE HEHYDSVTSRELRYLYENYKDNPNLKFTLKDHVIANPLKTLKTS ISESDLKSSPRRAROE $* * * * * * * * * * * * * * * * * * * * * * * * * * * * * * * * * * * * * * * * * * * * * * * * * * * * * * * * * * * *$

GPSLLORVRNLFDKSSSNKRSEKDTAOTSVGYRNTNIDIINDKTKGVNH IVENGVEVALT GPSLLQRVRNLFDKSSSNKRSEKDTAQTSVGYRNTNIDI INDKTKGVNH IVENGVEVALT GPSLLQRVRNLFDKSSSNKRSEKDTAOTSVGYRNTNIDI INDKTKGVNH IVENGVEVALT GPSLLQRVRNLFDKSSSNKRSEKDTAQTSVGYRNTNIDI INDKTKGVNH IVENGVEVALT GPSLLORVRNLFDKSSSNKRSEKDTAOTSVGYRNTNIDIINDKTKGVNHIVENGVEVALT GPSLLQRVRNLFDKSSSNKRSEKDTAQTSVGYRNTNIDIINDKTKGVNHIVENGVEVALT GPSLLQRVRNLFDKSSSNKRSEKDTAQTSVGYRNTNIDI INDKTKGVNH IVENGVEVALT GPSLLQRVRNLFDKSSSNKRSEKDTAOTSVGYRNTNIDI INDKTKGVNH IVENGVEVALT GPSLLQRVRNLFDKSSSNKRSEKDTAQTSVGYRNTNIDI INDKTKGVNH IVENGVEVALT GPSLLORVRNLFDKSSSNKRSEKDTAOTSVGYRNTNIDI INDKTKGVNH IVENGVEVALT GPSLLQRVRNLFDKSSSNKRSEKDTAQTSVGYRNTNIDI INDKTKGVNH IVENGVEVALT $\star * * * * * * * * * * * * * * * * * * * * * * * * * * * * * * * * * * * * * * * * * * * * * * * * * * * * * * * * * * * * * * * *$

NTDYKPLKLPNVEAAFKQTKLKAENIDPH IEAVKKLEI IASSANS IPKEHLLKALIEVTE NTDYKPLKLPNVEAAFKQTKLKAENIDPHIEAVKKLEI IASSANS IPKEHLLKALIEVTE NTDYKPLKLPNVEAAFKQTKLKAENIDPH IEAVKKLEI IAS SANS I PKEHLLKALIEVTE NTDYKPLKLPNVEAAFKOTKLKAENIDPH IEAVKKLEI IASSANS IPKEHLLKALIEVTE NTDYKPLKLPNVEAAFKQTKLKAENIDPH IEAVKKLEI IASSANS I PKEHLLKALIEVTE NTDYKPLKLPNVEAAFKQTKLKAENIDPH IEAVKKLEI IAS SANS IPKEHLLKALIEVTE NTDYKPLKLPNVEAAFKOTKLKAENIDPH IEAVKKLEI IASSANS I PKEHLLKALIEVTE NTDYKPLKLPNVEAAFKQTKLKAENIDPH IEAVKKLEI IASSANS IPKEHLLKALIEVTE NTDYKPLKLPNVEAAFKOTKLKAENIDPHIEAVKKLEI IASSANSIPKEHLLKALIEVTE NTDYKPLKLPNVEAAFKQTKLKAENIDPH IEAVKKLEI IASSANS IPKEHLLKALIEVTE NTDYKPLKLPNVEAAFKQTKLKAENIDPHIEAVKKLEI IASSANS I PKEHLLKALIEVTE $* * * * * * * * * * * * * * * * * * * * * * * * * * * * * * * * * * * * * * * * * * * * * * * * * * * * * * * * * * * * * *$

GKTDEDINVYOKLFNTRONISNEVAPTYSLRNLDGKDGKOILRSVAEIYKNLPLSDTYOA GKTDEDINVYQKLFNTRQNISNEVAPTYSLRNLDGKDGKQILRSVAEIYKNLPLSDTYQA GKTDEDINVYQKLFNTRQNISNEVAPTYSLRNLDGKDGKQILRSVAEIYKNLPLSDTYQA GKTDEDINVYQKLFNTRQNISNEVAPTYSLRNLDGKDGKQILRSVAEIYKNLPLSDTYQA GKTDEDINVYQKLFNTRQNISNEVAPTYSLRNLDGKDGKQILRSVAEIYKNLPLSDTYQA GKTDEDINVYOKLFNTRONISNEVAPTYSLRNLDGKDGKOILRSVAEIYKNLPLSDTYQA GKTDEDINVYQKLFNTRQNISNEVAPTYSLRNLDGKDGKQILRSVAEIYKNLPLSDTYQA GKTDEDINVYOKLFNTRONISNEVAPTYSLRNLDGKDGKOILRSVAEIYKNLPLSDTYOA GKTDEDINVYQKLFNTRQNISNEVAPTYSLRNLDGKDGKQILRSVAEIYKNLPLSDTYQA GKTDEDINVYQKLFNTRQNISNEVAPTYSLRNLDGKDGKQILRSVAEIYKNLPLSDTYQA GKTDEDINVYOKLFNTRONISNEVAPTYSLRNLDGKDGKOILRSVAEIYKNLPLSDTYOA $* * * * * * * * * * * * * * * * * * * * * * * * * * * * * * * * * * * * * * * * * * * * * * * * * * * * * * * * * * * *$

VRNYVNNRLIEKLSSNRLLLEHLANSKISGNEYAIKYIFDTVSRAKOEIFEQELNTELAP VRNYVNNRLIEKLSSNRLLLEHLANSKISGNEYAIKY IFDTVSRAKQEIFEQELNTELAP VRNYVNNRLIEKLSSNRLLLEHLANSKISGNEYAIKYIFDTVSRAKQEIFEQELNTELAP VRNYVNNRLIEKLSSNRLLLEHLANSKISGNEYAIKYIFDTVSRAKQEIFEQELNTELAP VRNYVNNRLIEKLSSNRLLLEHLANSKISGNEYAIKY IFDTVSRAKOEIFEQELNTELAP VRNYVNNRLIEKLSSNRLLLEHLANSKISGNEYAIKYIFDTVSRAKOEIFEQELNTELAP VRNYVNNRLIEKLSSNRLLLEHLANSKISGNEYAIKY IFDTVSRAKOEIFEOELNTELAP VRNYVNNRLIEKLSSNRLLLEHLANSKISGNEYAIKY IFDTVSRAKQEIFEQELNTELAP VRNYVNNRLIEKLSSNRLLLEHLANSKISGNEYAIKY IFDTVSRAKOE IFEQELNTELAP VRNYVNNRLIEKLSSNRLLLEHLANSKISGNEYAIKYIFDTVSRAKOEIFEQELNTELAP VRNYVNNRLIEKLSSNRLLLEHLANSKISGNEYAIKYIFDTVSRAKQEIFEQELNTELAP $* * * * * * * * * * * * * * * * * * * * * * * * * * * * * * * * * * * * * * * * * * * * * * * * * * * * * * * * * * * *$

VSLDIMRRKAANILSSEHGSYKDGTLS IYDKPVKSAFHSRLKNNGEVLNTIVHELTHHEQ VSLDIMRRKAANILSSEHGSYKDGTLS I YDKPVKSAFHSRLKNNGEVLNTIVHELTHHEQ VSLDIMRRKAANILSSEHGSYKDGTLS IYDKPVKSAFHSRLKNNGEVLNTIVHELTHHEQ VSLDIMRRKAANILSSEHGSYKDGTLS IYDKPVKSAFHSRLKNNGEVLNTIVHELTHHEQ VSLDIMRRKAANILSSEHGSYKDGTLS I YDKPVKSAFHSRLKNNGEVLNTIVHELTHHEQ VSLDIMRRKAANILSSEHGSYKDGTLS IYDKPVKSAFHSRLKNNGEVLNTIVHELTHHEQ VSLDIMRRKAANILSSEHGSYKDGTLSIYDKPVKSAFHSRLKNNGEVLNTIVHELTHHEQ VSLDIMRRKAANILSSEHGSYKDGTLSIYDKPVKSAFHSRLKNNGEVLNTIVHELTHHEQ VSLDIMRRKAANILSSEHGSYKDGTLS IYDKPVKSAFHSRLKNNGEVLNTIVHELTHHEO VSLDIMRRKAANILSSEHGSYKDGTLS IYDKPVKSAFHSRLKNNGEVLNTIVHELTHHEQ VSLDIMRRKAANILSSEHGSYKDGTLSIYDKPVKSAFHSRLKNNGEVLNTIVHELTHHEQ $* * * * * * * * * * * * * * * * * * * * * * * * * * * * * * * * * * * * * * * * * * * * * * * * * * * * * * * * * * * *$

DALAKIIDNKGYDAKLFDKNNILYITGGLGYPKOALERDAFLSGDSVSEAFMKKAKEYHE DALAKI IDNKGYDAKLFDKNNILYITGGLGYPKQALERDAFLSGDSVSEAFMKKAKEYHE 
NZS2 DALAKIIDNKGYDAKLFDKNNILYITGGLGYPKQALERDAFLSGDSVSEAFMKKAKEYHE DALAKI IDNKGYDAKLFDKNNILYITGGLGYPKOALERDAFLSGDSVSEAFMKKAKEYHE DALAKI IDNKGYDAKLFDKNNILYITGGLGYPKQALERDAFLSGDSVSEAFMKKAKEYHE DALAKI IDNKGYDAKLFDKNNILYITGGLGYPKOALERDAFLSGDSVSEAFMKKAKEYHE DALAKI IDNKGYDAKLFDKNN ILYITGGLGYPKQALERDAFLSGDSVSEAFMKKAKEYHE DALAKI IDNKGYDAKLFDKNNILYITGGLGYPKQALERDAFLSGDSVSEAFMKKAKEYHE DALAKI IDNKGYDAKLFDKNNILYITGGLGYPKQALERDAFLSGDSVSEAFMKKAKEYHE DALAKI IDNKGYDAKLFDKNNILYITGGLGYPKQALERDAFLSGDSVSEAFMKKAKEYHE DALAKI IDNKGYDAKLFDKNNILYITGGLGYPKQALERDAFLSGDSVSEAFMKKAKEYHE $* * * * * * * * * * * * * * * * * * * * * * * * * * * * * * * * * * * * * * * * * * * * * * * * * * * * * * * * * * * *$

$35000 \mathrm{HP}$

NZS1

NZS2

NZS 3

NZS 4

NZV1

82-029362

6644

HD 183

HMC 46

HMC 56

RTKQERKDAKKDEARIAKLYKQWEQEEANKKSASLNGSSQSLDSRSEVEFNRVSHKSVR * RTKQERKDAKKDEARIAKLYKQWEQEEANKKSASLNGSSQSLDSRSEVEFNRVSHKSVR * RTKOERKDAKKDEARIAKLYKOWEQEEANKKSASLNGSSQSLDSRSEVEFNRVSHKSVR * RTKQERKDAKKDEARIAKLYKQWEQEEANKKSASLNGSSQSLDSRSEVEFNRVSHKSVR * RTKQERKDAKKDEARIAKLYKQWEQEEANKKSASLNGSSQSLDSRSEVEFNRVSHKSVR * RTKQERKDAKKDEARIAKLYKQWEQEEANKKSASLNGSSQSLDSRSEVEFNRVSHKSVR * RTKQERKDAKKDEARIAKLYKQWEQEEANKKSASLNGSSQSLDSRSEVEFNRVSHKSVR * RTKQERKDAKKDEARIAKLYKOWEQEEANKKSASLNGSSOSLDSRSEVEFNRVSHKSVR * RTKQERKDAKKDEARIAKLYKQWEQEEANKKSASLNGSSQSLDSRSEVEFNRVSHKSVR * RTKQERKDAKKDEARIAKLYKQWEQEEANKKSASLNGSSQSLDSRSEVEFNRVSHKSVR * RTKQERKDAKKDEARIAKLYKQWEQEEANKKSASLNGSSQSLDSRSEVEFNRVSHKSVR* $* * * * * * * * * * * * * * * * * * * * * * * * * * * * * * * * * * * * * * * * * * * * * * * * * * * * * * * * * * * *$

Note: Due to difficulty in sorting the reads of 1 spA1 and IspA2 genes of class II strains, LspA2 sequences of class II strains were excluded from this analysis.

\section{4) LuxS (HD0370)}

$35000 \mathrm{HP}$

NZS 1

NZS2

NZS 3

NZS4

NZV1

$82-029362$

6644

HMC 46

HMC 56

33921

CIP 542

DMC 64

DMC 111

$35000 \mathrm{HP}$

NZS 1

NZS2

NZS3

NZS4

NZV1

82-029362

6644

HMC 46

HMC 56

33921

CIP 542

DMC 64

DMC 111

$35000 \mathrm{HP}$

NZS 1

NZS2

NZS3

NZS4

NZV1

82-029362

6644

HMC 46

HMC 56

33921
MPLLDSFKVDHTRMNAPAVRVAKTITTPKGDLITVFDLRFCRPNME IMSSKGIHTLEHLY MPLLDSFKVDHTRMNAPAVRVAKTITTPKGDLITVFDLRFCRPNME IMSSKGIHTLEHLY MPLLDSFKVDHTRMNAPAVRVAKTITTPKGDLITVFDLRFCRPNME IMSSKGIHTLEHLY MPLLDSFKVDHTRMNAPAVRVAKTITTPKGDLITVFDLRFCRPNMEIMSSKGIHTLEHLY MPLLDSFKVDHTRMNAPAVRVAKTITTPKGDLITVFDLRFCRPNME IMSSKGIHTLEHLY MPLLDSFKVDHTRMNAPAVRVAKTITTPKGDLITVFDLRFCRPNMEIMSSKGIHTLEHLY MPLLDSFKVDHTRMNAPAVRVAKTITTPKGDLITVFDLRFCRPNME IMSSKGIHTLEHLY MPLLDSFKVDHTRMNAPAVRVAKTITTPKGDLITVFDLRFCRPNME IMSSKG I HTLEHLY MPLLDSFKVDHTRMNAPAVRVAKTITTPKGDLITVFDLRFCRPNMEIMSSKGIHTLEHLY MPLLDSFKVDHTRMNAPAVRVAKTITTPKGDLITVFDLRFCRPNME IMSSKGIHTLEHLY MPLLDSFKVDHTRMNAPAVRVAKTITTPKGDLITVFDLRFCRPNME IMSSKGIHTLEHLY MPLLDSFKVDHTRMNAPAVRVAKTITTPKGDLITVFDLRFCRPNME IMSSKGIHTLEHLY MPLLDSFKVDHTRMNAPAVRVAKTITTPKGDLITVFDLRFCRPNMEIMSSKGIHTLEHLY MPLLDSFKVDHTRMNAPAVRVAKTITTPKGDLITVFDLRFCRPNME IMSSKGIHTLEHLY $* * * * * * * * * * * * * * * * * * * * * * * * * * * * * * * * * * * * * * * * * * * * * * * * * * * * * * * * * * * *$

AGFMRDHLNSDKVEI IDISPMGCRTGFYMSLIGEPSAOAVANAWKNAMHDILTKVSDVTQ AGFMRDHLNSDKVEI IDISPMGCRTGFYMSLIGEPSAQAVANAWKNAMHDILTKVSDVTQ AGFMRDHLNSDKVEIIDISPMGCRTGFYMSLIGEPSAQAVANAWKNAMHDILTKVSDVTQ AGFMRDHLNSDKVEIIDISPMGCRTGFYMSLIGEPSAQAVANAWKNAMHDILTKVSDVTQ AGFMRDHLNSDKVEI IDISPMGCRTGFYMSLIGEPSAQAVANAWKNAMHDILTKVSDVTQ AGFMRDHLNSDKVEI IDISPMGCRTGFYMSLIGEPSAQAVANAWKNAMHDILTKVSDVTQ AGFMRDHLNSDKVEIIDISPMGCRTGFYMSLIGEPSAQAVTNAWKNAMHDILTKVSDVTQ AGFMRDHLNSDKVEIIDISPMGCRTGFYMSLIGEPSAQAVTNAWKNAMHDILTKVSDVTQ AGFMRDHLNSDKVEIIDISPMGCRTGFYMSLIGEPSAQAVTNAWKNAMHDILTKVSDVTQ AGFMRDHLNSDKVEIIDISPMGCRTGFYMSLIGEPSAQAVTNAWKNAMHDILTKVSDVTQ AGFMRDHLNSDKVEI IDISPMGCRTGFYMSLIGEP SAQAVANAWKNAMHDILTKVSDVTQ AGFMRDHLNSDKVEI IDISPMGCRTGFYMSLIGEPSAQAVANAWKNAMHDILTKVSDVTQ AGFMRDHLNSDKVEI IDISPMGCRTGFYMSLIGEPSAQAVANAWKNAMHDILTKVSDVTQ AGFMRDHLNSDKVEIIDISPMGCRTGFYMSLIGEPSAQAVANAWKNAMHDILTKVSDVTQ $* * * * * * * * * * * * * * * * * * * * * * * * * * * * * * * * * * * * * * *: * * * * * * * * * * * * * * * * * * *$

IPELNIYQCGTFSEHSLEDAHQIARDVLAKGISVNLNEDLTLDEEWLNR * IPELNIYQCGTFSEHSLEDAHQIARDVLAKGISVNLNEDLTLDEEWLNR * IPELNIYQCGTFSEHSLEDAHQIARDVLAKGISVNLNEDLTLDEEWLNR * IPELNIYQCGTFSEHSLEDAHQIARDVLAKGISVNLNEDLTLDEEWLNR * IPELNIYQCGTFSEHSLEDAHQIARDVLAKGISVNLNEDLTLDEEWLNR * IPELNIYQCGTF SEHSLEDAHQIARDVLAKGISVNLNEDLTLDEEWLNR * IPELNIYQCGTFSEHSLEDAHQIARDVLAKGISVNLNEDLTLDEEWLNR * IPELNIYQCGTFSEHSLEDAHQIARDVLAKGISVNLNEDLTLDEEWLNR * IPELNIYQCGTFSEHSLEDAHQIARDVLAKGISVNLNEDLTLDEEWLNR * IPELNIYQCGTFSEHSLEDAHQIARDVLAKGISVNLNEDLTLDEEWLNR * IPELNIYQCGTFSEHSLEDAHQIARDVLAKGIS INLNEDLTLDEEWLNR * 
CIP542 IPELNIYQCGTFSEHSLEDAHQIARDVLAKGISINLNEDLTLDEEWLNR* DMC64 IPELNIYQCGTFSEHSLEDAHQIARDVLAKGIS INLNEDLTLDEEWLNR* DMC111 IPELNIYQCGTFSEHSLEDAHQIARDVLAKGIS INLNEDLTLDEEWLNR* $* * * * * * * * * * * * * * * * * * * * * * * * * * * * * * * * *: * * * * * * * * * * * * * * * *$

\section{5) $\operatorname{NcaA}(H D 1920)$}

33921

CIP5 42

DMC6 4

DMC 111

$35000 \mathrm{HP}$

82-0369

6644

HMC 46

HMC 56

33921

CIP5 42

DMC 64

DMC 111

$35000 \mathrm{HP}$

82-0369

6644

HMC 46

HMC 56

33921

CIP 542

DMC6 4

DMC 111

$35000 \mathrm{HP}$

82-0369

6644

HMC 46

HMC 56

33921

CIP 542

DMC 64

DMC 111

$35000 \mathrm{HP}$

82-0369

6644

HMC 46

HMC 56

33921

CIP 542

DMC6 4

DMC 111

$35000 \mathrm{HP}$

82-0369

6644

HMC 46

HMC 56

33921

CIP 542

DMC 64

DMC 111

$35000 \mathrm{HP}$

82-0369

6644

HMC 46

HMC 56
MKKIITILCLACGFSNFGHAIVTPGGIPPKCYEFLYPKGTNVLSKLOTTPNDNOLEOINL MKKI ITILCLACGF SNFGHAIVTPGGIPPKCYEFLYPKGTNVLSKLQTTPNDNQLEQINL MKKI ITILCLACGF SNFGHA IVTPGGIPPKCYEFLYPKGTNVLSKLQTTPNDNQLEQINL MKKIITILCLACGF SNFGHAIVTPGGIPPKCYEFLYPKGTNVLSKLQTTPNDNQLEQINL MKKIIIVSCLMSGVLNFGYSITTES--IPTCSSFL--------SKLLSTAKSDTLSKVKL MKKI I IVSCLMSGVLNFGYSITTES--IPTCSSFL-------SKLLSTAKSDTLSKVKL MKKIIIVSCLMSGVLNFGYSITTES--IPTCSSFL--------SKLLSTAKSDTLSKVKL MKKI I IVSCLMSGVLNFGYSITTES--IPTCSSFL-------SKLLSTAKSDTLSKVKL MKKIIIVSCLMSGVLNFGYSITTES--IPTCSSFL--------SKLLSTAKSDTLSKVKL

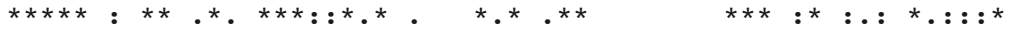

SDCKLDF---------PWSP--------LNTFADYPENRRIETLLDLWRVVRWNRGYL SDCKLDF---------PWSP--------LNTFADYPENRRIETLLDLWRVVRWNRGYL SDCKLDF---------PWSP---------LNTFADYPENRRIETLLDLWRVVRWNRGYL SDCKLDF----------PWSP---------LNTFADYPENRRIETLLDLWRVVRWNRGYL ENCTFEEFKNNYDKDKFPVEFRNNQEEKSFYRHLKERDNYAGISNLYDMWKWVRFNRGFL ENCTFEEFKNNYDKDKFPVEFRNNQEEKSFYRHLKERDNYAGISNLYDMWKWVRFNRGFL ENCTFEEFKNNYDKDKFPVEFRNNQEEKSFYRHLKERDNYAGISNLYDMWKWVRFNRGFL ENCTFEEFKNNYDKDKFPVEFRNNQEEKSFYRHLKERDNYAGISNLYDMWKWVRFNRGFL ENCTFEEFKNNYDKDKFPVEFRNNQEEKSFYRHLKERDNYAGISNLYDMWKWVRFNRGFL .: . : : $\quad$ * : : : : *..**:*: **:*** QEHNANLYDLYQRMNALESLSG----------LGNSES IAEFTKTIYPLIG--------QEHNANLYDLYQRMNALESLSG----------LGNSESIAEFTKTIYPLIG--------QEHNANLYDLYQRMNALESLSG----------LGNSES IAEFTKTIYPLIG--------QEHNANLYDLYQRMNALESLSG----------LGNSES IAEFTKTIYPLIG--------EOONEKLFHVYDKLNEVGINPESLDSMSYLNLKGINENINALTES INPSVEAIKYLLPDF EQQNEKLFHVYDKLNEVGINPESLDSMSYLNLKRINENMNALTES INPSVEAIKYLLPDF EQQNEKLFHVYDKLNEVGINPESLDSMSYLNLKRINENMNALTES INPSVEAIKYLLPDF EOQNEKLFHVYDKLNEVGINPESLDSMSYLNLKR INENMNALTES INPSVEAIKYLLPDF EQQNEKLFHVYDKLNEVGINPESLDSMSYLNLKRINENMNALTES INPSVEAIKYLLPDF : : : :*:.:*:: :* : .*.: :*::** :

----------------------------GEILKQVNQKVHELRKETYMNTANTAAM ------------------------------GEILKQVNQKVHELRKETYMNTANTAAM ------------------------------GEILKQVNQKVHELRKETYMNTANTAAM ---------------------------GEILKQVNQKVHELRKETYMNTANTAAM EKYVAAYEKLAKEGDSLSYSDFKKASGRGATTANIQQIDQRILHQFRKEMHMNTANTAAM EKYVAAYEKLAKEGDSLSYSDFKKASGRGATTANIQQIDQRILHQFRKEMHMNTANTAAM EKYVAAYEKLAKEGDSLSYSDFKKASGRGATTANIQQIDQRILHQFRKEMHMNTANTAAM EKYVAAYEKLAKEGDSLSYSDFKKASGRGATTANIQQIDQRILHQFRKEMHMNTANTAAM EKYVAAYEKLAKEGDSLSYSDFKKASGRGATTANIQQIDQRILHOFRKEMHMNTANTAAM $: *:::: *:: * * *: * * * * * * * *$

SSLNFGNSQGISFGAAIGGHKGQHSLALGTAYTDYQTQVNVKIALPVRQPKPSNITYGIG SSLNFGNSQGISFGAAIGGHKGQHSLALGTAYTDYQTQVNVKIALPVRQPKPSNITYGIG SSLNFGNSQGISFGAAIGGHKGQHSLALGTAYTDYQTQVNVKIALPVRQPKPSNITYGIG SSLNFGNSQGISFGAAIGGHKGQHSLALGTAYTDYQTQVNVKIALPVRQPKPSNITYGIG SSLNFGNGYGVSVGAAIGGHKGQYSLALGTAYTDYQTQVNVKIALPVKQPKPSNITYGVG SSLNFGNGYGVSVGAAIGGHKGQYSLALGTAYTDYQTQVNVKIALPVKQPKPSNITYGVG SSLNFGNGYGVSVGAAIGGHKGOYSLALGTAYTDYOTOVNVKIALPVKOPKPSNITYGVG SSLNFGNGYGVSVGAAIGGHKGQYSLALGTAYTDYQTQVNVKIALPVKQPKPSNITYGVG SSLNFGNGYGVSVGAAIGGHKGQYSLALGTAYTDYQTQVNVKIALPVKQPKPSNITYGVG $* * * * * * * . *: * . * * * * * * * * * *: * * * * * * * * * * * * * * * * * * * * * * *: * * * * * * * * * *: *$

FVYNFQ* FVYNFQ* FVYNFQ* FVYNFQ* FVYNFQ* FVYNFQ* FVYNFQ* FVYNFQ* FVYNFQ* $* * * * * * *$ 
Note: The NcaA sequence of NZS1, NZS2, NZS3, NZS4, and NZV1 were identical to that of $35000 \mathrm{HP}$.

\section{6) PAL (HD1772)}

$5000 \mathrm{HP}$ MKKIAKVLMIAAPAFVLTACSSSSGKTDANANMNGDAMAVNOFGGMTTEDLQTRYNTVYF

33921 MKKIAKVLMIAAPAFVLTACSSSSGKTDANANMNGDAMAVNQFGGMTSEDLQTRYNTVYF CIP542 MKKIAKVLMIAAPAFVLTACSSSSGKTDANANMNGDAMAVNQFGGMTSEDLQTRYNTVYF DMC64 MKKIAKVLMIAAPAFVLTACSSSSGKTDANANMNGDAMAVNQFGGMTSEDLQTRYNTVYF DMC111 MKKIAKVLMIAAPAFVLTACSSSSGKTDANANMNGDAMAVNQFGGMTSEDLQTRYNTVYF $* * * * * * * * * * * * * * * * * * * * * * * * * * * * * * * * * * * * * * * * * * * * * * *: * * * * * * * * * * * *$

35000HP NFDSYAVEGEYROLLDAHAAYLTSSNSKVTVTGHADERGTPEYNIALGQRRADAVKNYLA 33921 NFDSYAVEGEYRQLLDAHAAYLTSSNSKVTVTGHADERGTPEYNIALGQRRADAVKNYLA CIP54 2 NFDSYAVEGEYRQLLDAHAAYLTSSNSKVTVTGHADERGTPEYNIALGQRRADAVKNYLA DMC64 NFDSYAVEGEYRQLLDAHAAYLTSSNSKVTVTGHADERGTPEYNIALGQRRADAVKNYLA DMC111 NFDSYAVEGEYRQLLDAHAAYLTSSNSKVTVTGHADERGTPEYNIALGQRRADAVKNYLA $* * * * * * * * * * * * * * * * * * * * * * * * * * * * * * * * * * * * * * * * * * * * * * * * * * * * * * * * * * * *$

35000HP TKGVSOVSTVSYGEEKPSVLGHTEADYAKNRRAVLEY* 33921 TKGVSOVSTVSYGEEKPSVLGHTEADYAKNRRAVLEY* CIP54 2 TKGVSOVSTVSYGEEKPSVLGHTEADYAKNRRAVLEY* DMC64 TKGVSOVSTVSYGEEKPSVLGHTEADYAKNRRAVLEY* DMC111 TKGVSQVSTVSYGEEKPSVLGHTEADYAKNRRAVLEY * $* * * * * * * * * * * * * * * * * * * * * * * * * * * * * * * * * * * * * *$

Note: The PAL sequence of NZS1, NZS2, NZS3, NZS4, NZV1, 82-029362, 6644, HD183, HMC46, and HMC56 were identical to that of $35000 \mathrm{HP}$.

\section{7) RelA (HD1185)}

33921

CIP5 42

DMC 64

DMC 111

NZV1

$35000 \mathrm{HP}$

NZS 1

NZS2

NZS 3

NZS 4

82-029362

6644

HMC 46

HMC 56

33921

CIP5 42

DMC 64

DMC 111

NZV1

$35000 \mathrm{HP}$

NZS 1

NZS2

NZS 3

NZS 4

$82-029362$

6644

HMC 46

HMC 56

33921

CIP 542

DMC 64

DMC 111

NZV1

$35000 \mathrm{HP}$

NZS 1
MVAIRRSHELDPSTFELASWSASLQMSPITFEELQIAWRY IHEKLDTDSYHLMWDGVEMV MVAIRRSHELDPSTFELASWSASLQMSPITFEELQIAWRY I HEKLDTDSYHLMWDGVEMV MVAIRRSHELDPSTFELASWSASLQMSPITFEELQIAWRY I HEKLDTDSYHLMWDGVEMV MVAIRRSHELDPSTFELASWSASLOMSPITFEELOIAWRY I HEKLDTDSYHLMWDGVEMV MVAIRRSHELDPSTFELASWSASLQMSPITFEELQIAWRY IHEKLDTDSYHLMWDGVEMV MVAIRRSHELDPSTFELASWSASLQMSP ITFEELQIAWRY IHEKLDTDSYHLMWDGVEMV MVAIRRSHELDPSTFELASWSASLQMSPITFEELOIAWRY IHEKLDTDSYHLMWDGVEMV MVAIRRSHELDPSTFELASWSASLQMSPITFEELQIAWRY I HEKLDTDSYHLMWDGVEMV MVAIRRSHELDPSTFELASWSASLQMSPITFEELQIAWRY I HEKLDTDSYHLMWDGVEMV MVAIRRSHELDPSTFELASWSASLQMSPITFEELQIAWRY I HEKLDTDSYHLMWDGVEMV MVAIRRSHELDPSTFELASWSASLQMSPITFEELQIAWRY IHEKLDTDSYHLMWDGVEMV MVAIRRSHELDPSTFELASWSASLQMSP ITFEELQIAWRY I HEKLDTDSYHLMWDGVEMV MVAIRRSHELDPSTFELASWSASLQMSPITFEELQIAWRY I HEKLDTDSYHLMWDGVEMV MVAIRRSHELDPSTFELASWSASLQMSPITFEELQIAWRY I HEKLDTDSYHLMWDGVEMV $* * * * * * * * * * * * * * * * * * * * * * * * * * * * * * * * * * * * * * * * * * * * * * * * * * * * * * * * * * * *$

ELLHGLNMDDDSLVAALLFPLVKNNIVDLAQVKEEFSNQVKNLVKGVIEMDNIRQLNANS ELLHGLNMDDDSLVAALLFPLVKNNIVDLAQVKEEF SNQVKNLVKGVIEMDNIRQLNANS ELLHGLNMDDDSLVAALLFPLVKNNIVDLAOVKEEFSNOVKNLVKGVIEMDNIROLNANS ELLHGLNMDDDSLVAALLFPLVKNNIVDLAQVKEEFSNQVKNLVKGVIEMDNIRQLNANS ELLHGLNMDDDSLVAALLFPLVKNNIVDLAQVKEEF SNQVKNLVKGVIEMDNIRQLNANS ELLHGLNMDDDSLVAALLFPLVKNNIVDLAQVKEEF SNQVKNLVKGVIEMDNIRQLNASS ELLHGLNMDDDSLVAALLFPLVKNNIVDLAQVKEEF SNQVKNLVKGVIEMDNIRQLNANS ELLHGLNMDDDSLVAALLFPLVKNNIVDLAQVKEEFSNQVKNLVKGVIEMDNIRQLNANS ELLHGLNMDDDSLVAALLFPLVKNNIVDLAQVKEEF SNQVKNLVKGVIEMDNIRQLNANS ELLHGLNMDDDSLVAALLFPLVKNNIVDLAQVKEEF SNQVKNLVKGVIEMDNIRQLNANS ELLHGLNMDDDSLVAALLFPLVKNNIVDLAQVKEEF SNQVKNLVKGVIEMDNIRQLNANS ELLHGLNMDDDSLVAALLF PLVKNNIVDLAQVKEEF SNOVKNLVKGVIEMDN IRQLNANS ELLHGLNMDDDSLVAALLFPLVKNNIVDLAQVKEEFSNOVKNLVKGVIEMDNIRQLNANS ELLHGLNMDDDSLVAALLFPLVKNNIVDLAQVKEEFSNQVKNLVKGVIEMDNIRQLNANS $\star * * * * * * * * * * * * * * * * * * * * * * * * * * * * * * * * * * * * * * * * * * * * * * * * * * * * * * * * * . *$

RSDFOIDNIRRMLLAMVDDFRCVVIKLAERIAYLREIHRYTEEDLVLAAKECSHIYAPLA RSDFOIDNIRRMLLAMVDDFRCVVIKLAERIAYLREIHRYTEEDLVLAAKECSHIYAPLA RSDFQIDNIRRMLLAMVDDFRCVVIKLAERIAYLREIHRYTEEDLVLAAKECSHIYAPLA RSDFQIDNIRRMLLAMVDDFRCVVIKLAERIAYLREIHRYTEEDLVLAAKECSHIYAPLA RSDFQIDNIRRMLLAMVDDFRCVVIKLAERIAYLREIHRYTEEDLVLAAKECSHIYAPLA RSDFOIDNIRRMLLAMVDDFRCVVIKLAERIAYLREIHRYTEEDLVLAAKECSHIYAPLA RSDFQIDNIRRMLLAMVDDFRCVVIKLAERIAYLREI HRYTEEDLVLAAKECSHIYAPLA 
NZS2

NZS3

NZS 4

82-029362

6644

HMC 46

HMC 56

33921

CIP 542

DMC 64

DMC 111

NZV1

$35000 \mathrm{HP}$

NZS 1

NZS2

NZS3

NZS 4

82-029362

6644

HMC 46

HMC 56

33921

CIP 542

DMC 64

DMC 111

NZV1

$35000 \mathrm{HP}$

NZS1

NZS2

NZS 3

NZS 4

82-029362

6644

HMC 46

HMC 56

33921

CIP5 42

DMC 64

DMC 111

NZV1

$35000 \mathrm{HP}$

NZS 1

NZS2

NZS3

NZS 4

82-029362

6644

HMC 46

HMC 56

33921

CIP 542

DMC 64

DMC 111

NZV1

$35000 \mathrm{HP}$

NZS 1

NZS2

NZS 3

NZS 4

82-029362

6644

HMC 46

HMC 56
RSDFQIDNIRRMLLAMVDDFRCVVIKLAERIAYLREIHRYTEEDLVLAAKECSHIYAPLA RSDFOIDNIRRMLLAMVDDFRCVVIKLAERIAYLREIHRYTEEDLVLAAKECSHIYAPLA RSDFQIDNIRRMLLAMVDDFRCVVIKLAERIAYLREIHRYTEEDLVLAAKECSHIYAPLA RSDFOIDNIRRMLLAMVDDFRCVVIKLAERIAYLREIHRYTEEDLVLAAKECSHIYAPLA RSDFQIDNIRRMLLAMVDDFRCVVIKLAERIAYLREIHRYTEEDLVLAAKECSHIYAPLA RSDFQIDNIRRMLLAMVDDFRCVVIKLAERIAYLREI HRYTEEDLVLAAKECSHIYAPLA RSDFQIDNIRRMLLAMVDDFRCVVIKLAERIAYLREIHRYTEEDLVLAAKECSHIYAPLA $* * * * * * * * * * * * * * * * * * * * * * * * * * * * * * * * * * * * * * * * * * * * * * * * * * * * * * * * * * * *$

NRLGIGQLKWELEDYCFRVLHPQSYRRIATQLGERRLERENYIANFVSQLTASLTEEVDT NRLGIGQLKWELEDYCFRVLHPOSYRRIATOLGERRLERENYIANFVSOLTASLTEEVDT NRLGIGQLKWELEDYCFRVLHPQSYRRIATQLGERRLERENYIANFVSQLTASLTEEVDT NRLGIGQLKWELEDYCFRVLHPQSYRRIATQLGERRLERENYIANFVSQLTASLTEEVDT NRLGIGQLKWELEDYCFRVLHPQSYRRIATQLGERRLERENYIANFVSQLTASLTEEVDT NRLGIGQLKWELEDYCFRVLHPQSYRRIATQLGERRLERENYIANFVSQLTASLTEEVDT NRLGIGOLKWELEDYCFRVLHPOSYRRIATOLGERRLERENYIANFVSOLTASLTEEVDT NRLGIGQLKWELEDYCFRVLHPQSYRRIATQLGERRLERENYIANFVSQLTASLTEEVDT NRLGIGQLKWELEDYCFRVLHPQSYRRIATQLGERRLERENYIANFVSQLTASLTEEVDT NRLGIGQLKWELEDYCFRVLHPQSYRRIATQLGERRLERENYIANFVSOLTASLTEEVDT NRLGIGQLKWELEDYCFRVLHPQSYRRIATQLGERRLERENYIANFVSQLTASLTEEVDT NRLGIGQLKWELEDYCFRVLHPQSYRRIATQLGERRLERENYIANFVSQLTASLTEEVDT NRLGIGQLKWELEDYCFRVLHPQSYRRIATQLGERRLERENYIANFVSQLTASLTEEVDT NRLGIGQLKWELEDYCFRVLHPQSYRRIATQLGERRLERENYIANFVSQLTASLTEEVDT $* * * * * * * * * * * * * * * * * * * * * * * * * * * * * * * * * * * * * * * * * * * * * * * * * * * * * * * * * * * *$

LEVYGRPKH IYS IWKKMQKKNVQFEQLFDIRAVRVIVPNVQDCYSVLGI I HTQYKHLPEH LEVYGRPKH IYS IWKKMQKKNVQFEQLFDIRAVRVIVPNVQDCYSVLGI I HTQYKHLPEH LEVYGRPKH IYS IWKKMOKKNVOFEOLFDIRAVRVIVPNVODCYSVLGI I HTOYKHLPEH LEVYGRPKHIYSIWKKMQKKNVQFEQLFDIRAVRVIVPNVQDCYSVLGI I HTQYKHLPEH LEVYGRPKH IYS IWKKMQKKNVQFEQLFDIRAVRVIVPNVQDCYSVLGI I HTQYKHLPEH LEVYGRPKH IYS IWKKMQKKNVQFEQLFDIRAVRVIVPNVQDCYSVLGI I HTQYKHLPEH LEVYGRPKH IYS IWKKMQKKNVQFEQLFDIRAVRVIVPNVQDCYSVLGI I HTQYKHLPEH LEVYGRPKH IYS IWKKMQKKNVQFEQLFDIRAVRVIVPNVQDCYSVLGI I HTQYKHLPEH LEVYGRPKH IYS IWKKMQKKNVQFEQLFDIRAVRVIVPNVQDCYSVLGI I HTQYKHLPEH LEVYGRPKH IYS IWKKMQKKNVQFEQLFDIRAVRVIVPNVQDCYSVLGI I HTQYKHLPEH LEVYGRPKHIYSIWKKMQKKNVQFEQLFDIRAVRVIVPNVQDCYSVLGI I HTQYKHLPEH LEVYGRPKHIYS IWKKMQKKNVQFEQLFDIRAVRVIVPNVQDCYSVLGI I HTQYKHLPEH LEVYGRPKH IYS IWKKMOKKNVQFEQLFDIRAVRVIVPNVQDCYSVLGI I HTQYKHLPEH LEVYGRPKH IYS IWKKMQKKNVQFEQLFDIRAVRVIVPNVQDCYSVLGI I HTQYKHLPEH $* * * * * * * * * * * * * * * * * * * * * * * * * * * * * * * * * * * * * * * * * * * * * * * * * * * * * * * * * * * *$

FDDYIAHPKPNGYQSIHTVVLGEGEQPIEVQIRTRKMHDEAELGIAAHWKYKEGQTVSRS FDDYIAHPKPNGYQSIHTVVLGEGEQPIEVQIRTRKMHDEAELGIAAHWKYKEGQTVSRS FDDYIAHPKPNGYQS I HTVVLGEGEQP IEVQIRTRKMHDEAELGIAAHWKYKEGQTVSRS FDDYIAHPKPNGYQS IHTVVLGEGEQP IEVQIRTRKMHDEAELGIAAHWKYKEGQTVSRS FDDYIAHPKPNGYQS IHTVVLGEGEQPIEVQIRTRKMHDEAELGIAAHWKYKEGPTVSRS FDDYIAHPKPNGYQS IHTVVLGEGEQPIEVQIRTRKMHDEAELGIAAHWKYKEGPTVSRS FDDYIAHPKPNGYQS IHTVVLGEGEQP IEVQIRTRKMHDEAELGIAAHWKYKEGPTVSRS FDDYIAHPKPNGYQS I HTVVLGEGEQPIEVQIRTRKMHDEAELGIAAHWKYKEGPTVSRS FDDYIAHPKPNGYQS IHTVVLGEGEQP IEVQIRTRKMHDEAELGIAAHWKYKEGPTVSRS FDDYIAHPKPNGYQS IHTVVLGEGEQPIEVQIRTRKMHDEAELGIAAHWKYKEGPTVSRS FDDYIAHPKPNGYQSIHTVVLGEGEQPIEVQIRTRKMHDEAELGIAAHWKYKEGPTVSRS FDDYIAHPKPNGYQSIHTVVLGEGEQPIEVQIRTRKMHDEAELGIAAHWKYKEGPTVSRS FDDYIAHPKPNGYQSIHTVVLGEGEQPIEVQIRTRKMHDEAELGIAAHWKYKEGPTVSRS FDDYIAHPKPNGYQS I HTVVLGEGEQPIEVQIRTRKMHDEAELGIAAHWKYKEGPTVSRS $* * * * * * * * * * * * * * * * * * * * * * * * * * * * * * * * * * * * * * * * * * * * * * * * * * * * * * * * * *$

GYEEKI IWLRKLLAWQNDLAASSDMMAEMRSQIFDDRVYVFTPRGEVIDLPKNSTPLDFA GYEEKI IWLRKLLAWQNDLAASSDMMAEMRSQIFDDRVYVFTPRGEVIDLPKNSTPLDFA GYEEKI IWLRKLLAWQNDLAASSDMMAEMRSOIFDDRVYVFTPRGEVIDLPKNSTPLDFA GYEEKI IWLRKLLAWQNDLAAS SDMMAEMRSQIFDDRVYVFTPRGEVIDLPKNSTPLDFA GYEEKI IWLRKLLAWQNDLAVSSDMMADMRSQIFDDRVYVFTPRGEVIDLPKNSTPLDFA GYEEKI IWLRKLLAWQNDLAVSSDMMADMRSQIFDDRVYVFTPRGEVIDLPKNSTPLDFA GYEEKI IWLRKLLAWQNDLAVSSDMMADMRSQIFDDRVYVFTPRGEVIDLPKNSTPLDFA GYEEKI IWLRKLLAWQNDLAVSSDMMADMRSQIFDDRVYVFTPRGEVIDLPKNSTPLDFA GYEEKI IWLRKLLAWQNDLAVSSDMMADMRSQIFDDRVYVFTPRGEVIDLPKNSTPLDFA GYEEKI IWLRKLLAWQNDLAVSSDMMADMRSQIFDDRVYVFTPRGEVIDLPKNSTPLDFA GYEEKI IWLRKLLAWQNDLAVSSDMMADMRSQIFDDRVYVFTPRGEVIDLPKNSTPLDFA GYEEKI IWLRKLLAWONDLAVSSDMMADMRSOIFDDRVYVFTPRGEVIDLPKNSTPLDFA GYEEKI IWLRKLLAWQNDLAVSSDMMADMRSQIFDDRVYVFTPRGEVIDLPKNSTPLDFA GYEEKI IWLRKLLAWQNDLAVSSDMMADMRSQIFDDRVYVFTPRGEVIDLPKNSTPLDFA $* * * * * * * * * * * * * * * * * * * * . * * * * * *: * * * * * * * * * * * * * * * * * * * * * * * * * * * * * * * *$ 
33921

CIP 542

DMC 64

DMC 111

NZV1

$35000 \mathrm{HP}$

NZS1

NZS2

NZS3

NZS4

82-029362

6644

HMC 46

HMC 56

33921

CIP 542

DMC6 4

DMC 111

NZV1

$35000 \mathrm{HP}$

NZS 1

NZS2

NZS 3

NZS 4

82-029362

6644

HMC 46

HMC 56

33921

CIP5 42

DMC 64

DMC 111

NZV1

$35000 \mathrm{HP}$

NZS 1

NZS2

NZS3

NZS 4

82-029362

6644

HMC 46

HMC 56

33921

CIP 542

DMC 64

DMC 111

NZV1

$35000 \mathrm{HP}$

NZS1

NZS2

NZS3

NZS4

82-029362

6644

HMC 46

HMC 56

33921

CIP 542

DMC6 4

DMC 111

NZV1

$35000 \mathrm{HP}$

NZS1

NZS2

NZS 3
YAVHSEIGHRCIGAKIADRIVPFTYVLQMGDQVEI ITQKTPNPSRDWLSPQAGFVNTSKA YAVHSEIGHRCIGAKIADR IVPFTYVLQMGDQVEI ITQKTPNPSRDWLSPQAGFVNTSKA YAVHSE IGHRC IGAKIADR IVPFTYVLQMGDQVEI ITQKTPNPSRDWLSPQAGFVNTSKA YAVHSE IGHRC I GAKIADR IVPFTYVLOMGDOVE I ITOKTPNPSRDWLSPOAGFVNTSKA YAVHSEIGHRCIGAKIADR IVPFTYVLQMGDQVEI ITQKTPNPSRDWLSPQAGFVNTSKA YAVHSEIGHRCIGAKIADRIVPFTYVLQMGDQVEI ITQKTPNPSRDWLSPQAGFVNTSKA YAVHSE IGHRCIGAK IADR IVPFTYVLOMGDOVE I ITOKTPNPSRDWLSPOAGFVNTSKA YAVHSEIGHRCIGAKIADRIVPFTYVLQMGDQVEI ITQKTPNPSRDWLSPQAGFVNTSKA YAVHSEIGHRCIGAKIADRIVPFTYVLQMGDQVEIITQKTPNPSRDWLSPQAGFVNTSKA YAVHSEIGHRCIGAKIADR IVPFTYVLQMGDQVEI ITQKTPNPSRDWLSPQAGFVNTSKA YAVHSEIGHRCIGAKIADR IVPFTYVLOMGDOVEI ITOKTPNPSRDWLSPOAGFVNTSKA YAVHSEIGHRCIGAKIADRIVPFTYVLQMGDQVEI ITQKTPNPSRDWLSPQAGFVNTSKA YAVHSEIGHRCIGAKIADRIVPFTYVLQMGDQVEI ITQKTPNPSRDWLSPQAGFVNTSKA YAVHSEIGHRC IGAKIADR IVPFTYVLQMGDQVE I ITQKTPNPSRDWLSPQAGFVNTSKA $* * * * * * * * * * * * * * * * * * * * * * * * * * * * * * * * * * * * * * * * * * * * * * * * * * * * * * * * * * *$

RAKI IAWFKKLDREKNIPIGKEALEAEISRLGLSHKQIEQYALPRYNLKQFDDLYAAIGG RAKIIAWFKKLDREKNIPIGKEALEAEISRLGLSHKQIEQYALPRYNLKQFDDLYAAIGG RAKI IAWFKKLDREKNIP I GKEALEAE ISRLGLSHKQIEQYALPRYNLKQFDDLYAAIGG RAKIIAWF KKLDREKNIPIGKEALEAE ISRLGLSHKQIEQYALPRYNLKQFDDLYAAIGG RAKI IAWFKKLDREKNIPLGKEALEAEISRLGLSHKQIEQYALPRYNLKQFDDLYAAIGG RAKI IAWFKKLDREKNIPLGKEALEAEISRLGLSHKQIEQYALPRYNLKQFDDLYAAIGG RAKI IAWFKKLDREKNIPLGKEALEAEISRLGLSHKQIEQYALPRYNLKQFDDLYAAIGG RAKI IAWF KKLDREKNIPLGKEALEAE ISRLGLSHKQIEQYALPRYNLKQFDDLYAAIGG RAKIIAWFKKLDREKNIPLGKEALEAEISRLGLSHKQIEQYALPRYNLKQFDDLYAAIGG RAKI IAWFKKLDREKN I PLGKEALEAE ISRLGLSHKQIEQYALPRYNLKQFDDLYAAIGG RAKI IAWFKKLDREKNIPLGKEALEAEISRLGLSHKQIEQYALPRYNLKQFDDLYAAIGG RAKI IAWFKKLDREKNIPLGKEALEAEISRLGLSHKOIEOYALPRYNLKOFDDLYAAIGG RAKI IAWFKKLDREKNIPLGKEALEAE ISRLGLSHKQIEQYALPRYNLKQFDDLYAAIGG RAKI IAWFKKLDREKN IPLGKEALEAE ISRLGLSHKQIEQYALPRYNLKQFDDLYAAIGG $* * * * * * * * * * * * * * * * * *: * * * * * * * * * * * * * * * * * * * * * * * * * * * * * * * * * * * * * * * * *$

GDIRLNQLSHYLQSKLMKPTAEQEDEAVLKHVNKNAYNAQQQKGKNGQI I IDSVGNLMHN GDIRLNQLSHYLQSKLMKPTAEQEDEAVLKHVNKNAYNAQQQKGKNGQI I IDSVGNLMHN GDIRLNQLSHYLQSKLMKPTAEQEDEAVLKHVNKNAYNAQQQKGKNGQI I IDGVGNLMHN GDIRLNQLSHYLQSKLMKPTAEQEDEAVLKHVNKNAYNAQQQKGKNGQI I IDGVGNLMHN GDIRLNQLSHYLQSKLIKPTAEQEDEAVLKHVNKNAYNAQQQKGKNGQI I IDGVGNLMHN GDIRLNQLSHYLQSKLIKPTAEQEDEAVLKHVNKNAYNAQQQKGKNGQI I IDGVGNLMHN GDIRLNQLSHYLQSKLIKPTAEQEDEAVLKHVNKNAYNAQQQKGKNGQI I IDGVGNLMHN GDIRLNOLSHYLOSKL I KPTAEOEDEAVLKHVNKNAYNAOOOKGKNGOI I IDGVGNLMHN GDIRLNQLSHYLQSKLIKPTAEQEDEAVLKHVNKNAYNAQQQKGKNGQI I I DGVGNLMHN GDIRLNQLSHYLQSKLIKPTAEQEDEAVLKHVNKNAYNAQQQKGKNGQI I IDGVGNLMHN GDIRLNQLSHYLQSKLIKPTAEQEDEAVLKHVNKNAYNAQQQKGKNGQI I IDGVGNLMHN GDIRLNQLSHYLQSKLIKPTAEQEDEAVLKHVNKNAYNAQQQKGKNGQI I IDGVGNLMHN GDIRLNOLSHYLOSKLIKPTAEOEDEAVLKHVNKNAYNAOOOKGKNGOI I IDGVGNLMHN GDIRLNQLSHYLQSKLIKPTAEQEDEAVLKHVNKNAYNAQQQKGKNGQI I IDGVGNLMHN $* * * * * * * * * * * * * * * *: * * * * * * * * * * * * * * * * * * * * * * * * * * * * * * * * * * * . * * * * * * *$

LACCCQPIPGDDIVGYITQGRGISIHCANCEQLFELRSANPERIVSAQWDSHFKAGFSLR LACCCQP IPGDDIVGYITQGRG IS I HCANCEQLFELRSANPERIVSAQWDSHFKAGFSLR LACCCQPIPGDDIVGYITQGRGIS I HCANCEQLFELRSANPERIVSAQWDSHFKAGFSLR LACCCQP IPGDDIVGYITQGRGIS I HCANCEQLFELRSANPERIVSAQWDSHFKAGFSLR LARCCQP IPGDDIVGYITQGRGIS I HCANCEQLFELRSANPERIVSAQWDSHFKAGFSLR LARCCQPIPGDDIVGYITQGRGIS I HCANCEQLFELRSANPERIVSAQWDSHFKAGFSLR LARCCQP I PGDDIVGYITQGRGIS I HCANCEQLFELRSANPERIVSAQWDSHFKAGFSLR LARCCQPIPGDDIVGYITQGRGIS I HCANCEQLFELRSANPERIVSAQWDSHFKAGFSLR LARCCQP IPGDD IVGYITQGRGIS I HCANCEQLFELRSANPERIVSAQWDSHFKAGF SLR LARCCQPIPGDDIVGYITQGRGIS I HCANCEQLFELRSANPERIVSAQWDSHFKAGFSLR LARCCQPIPGDDIVGYITQGRGIS I HCANCEQLFELRSANPERIVSAQWDSHFKAGFSLR LARCCQP IPGDDIVGYITQGRGIS I HCANCEQLFELRSANPERIVSAQWDSHFKAGF SLR LARCCQPIPGDDIVGYITQGRGISIHCANCEQLFELRSANPERIVSAQWDSHFKAGFSLR LARCCQP IPGDDIVGYITQGRGIS I HCANCEQLFELRSANPERIVSAQWDSHFKAGFSLR ** $* * * * * * * * * * * * * * * * * * * * * * * * * * * * * * * * * * * * * * * * * * * * * * * * * * * * * * * *$

IRI IANECNGLLRDVSAVMANEKVNVLTVASRODI KRGLTVMDIELEMSNVEM------IRI IANECNGLLRDVSAVMANEKVNVLTVASRQDIKRGLTVMDIELEMSNVEM------IRI IANECNGLLRDVSAVMANEKVNVLTVASRQDIKRGLTVMDIELEMSNVEM-----IRI IANECNGLLRDVSAVMANEKVNVLTVASRQDIKRGLTVMDIELEMSNVEM------IRI IANERNGLLRDVSAVMANEKVNVLTVASRQDIKRGLTVMDIELEMSNVEMLAKILTR IRI IANERNGLLRDVSAVMANEKVNVLTVASRQDIKRGLTVMDIELEMSNVEML-----IRIIANERNGLLRDVSAVMANEKVNVLTVASRQDIKRGLTVMDIELEMSNVEML-----IRI IANERNGLLRDVSAVMANEKVNVLTVASRODIKRGLTVMDIELEMSNVEML-- - - IRIIANERNGLLRDVSAVMANEKVNVLTVASRQDIKRGLTVMDIELEMSNVEML------ 
NZS 4

82-029362

6644

HMC 46

HMC 56

33921

CIP 542

DMC 64

DMC 111

NZV1

$35000 \mathrm{HP}$

NZS 1

NZS2

NZS 3

NZS 4

82-029362

6644

HMC 46

HMC 56
IRI IANERNGLLRDVSAVMANEKVNVLTVASRODIKRGLTVMDIELEMSNVEML-----IRI IANERNGLLRDVSAVMANEKVNVLTVASRODIKRGLTVMDIELEMSNVEML-----IRI IANERNGLLRDVSAVMANEKVNVLTVASRQDIKRGLTVMDIELEMSNVEML-----IRI IANERNGLLRDVSAVMANEKVNVLTVASRODI KRGLTVMDIELEMSNVEML-----IRI IANERNGLLRDVSAVMANEKVNVLTVASRODIKRGLTVMDIELEMSNVEML------

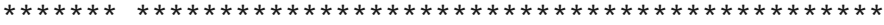

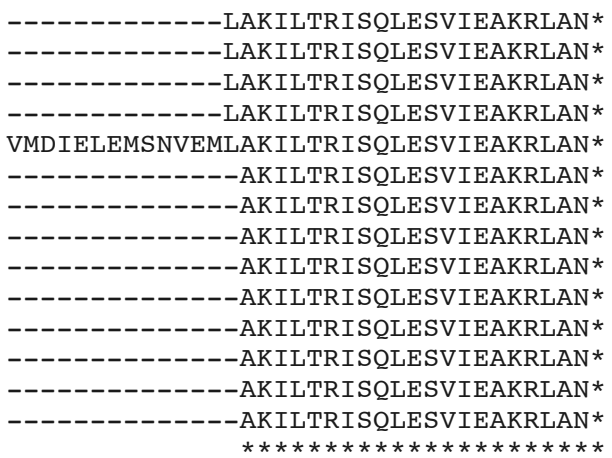

\section{8) SapA (HD1230)}

$35000 \mathrm{HP}$

$82-029362$

6644

HMC 46

HMC56

33921

CIP 542

DMC6 4

DMC 111

$35000 \mathrm{HP}$

82-029362

6644

HMC 46

HMC 56

33921

CIP 542

DMC 64

DMC 111

$35000 \mathrm{HP}$

82-029362

6644

HMC 46

HMC 56

33921

CIP 542

DMC 64

DMC 111

$35000 \mathrm{HP}$

82-029362

6644

HMC 46

HMC5 6

33921

CIP5 4

DMC 64

DMC 111

$35000 \mathrm{HP}$

82-029362
MKILALSILKFSPFFAVFCWISTAYSAPRVPKELSADSLIYCTS ISGLSFNPOKADVGTN MKILALSILKF SPFFAVFCWISTAYSAPRVPKELSADSLIYCTS ISGLSFNPQKADVGTN MKILALSILKFSPFFAVFCWISTAYSAPRVPKELSADSLIYCTS ISGLSFNPOKADVGTN MKILALSILKF SPFFAVFCWISTAYSAPRVPKELSADSLIYCTSISGLSFNPQKADVGTN MKILALSILKFSPFFAVFCWISTAYSAPRVPKELSADSLIYCTSISGLSFNPQKADVGTN MKILALSILKF SPFFAVFCWISTAYAAPRVPKELSADSLIYCTSISGLSFNPOKADVGTN MKILALSILKF SPFFAVFCWISTAYAAPRVPKELSADSLIYCTSISGLSFNPQKADVGTN MKILALSILKF SPFFAVFCWISTAYAAPRVPKELSADSLIYCTS ISGLSFNPQKADVGTN MKILALSILKF SPFFAVFCWISTAYAAPRVPKELSADSLIYCTSISGLSFNPQKADVGTN $\star * * * * * * * * * * * * * * * * * * * * * * * *: * * * * * * * * * * * * * * * * * * * * * * * * * * * * * * * * * *$

MNVVTEQIYDKLFEIDRHTHRVIPSLAETFSVSDDGKEITLNLRRQVAFHKTPWFTPTRI MNVVTEQIYDKLFEIDRHTHRVIPSLAETFSVSDDGKEITLNLRRQVAFHKTPWFTPTRL MNVVTEQIYDKLFEIDRHTHRVIPSLAETF SVSDDGKEITLNLRRQVAFHKTPWFTPTRL MNVVTEOIYDKLFEIDRHTHRVIPSLAETF SVSDDGKEITLNLRROVAFHKTPWFTPTRL MNVVTEQIYDKLFEIDRHTHRVIPSLAETF SVSDDGKEITLNLRRQVAFHKTPWFTPTRL MNVVTEQIYDKLFEIDRHTHRVIPSLAETF SVSDDGKEITLNLRRQVAFHKTPWFTPTRL MNVVTEOIYDKLFEIDRHTHRVIPSLAETFSVSDDGKEITLNLRROVAFHKTPWFTPTRL MNVVTEQI YDKLFEIDRHTHRVIPSLAETF SVSDDGKEITLNLRRQVAFHKTPWFTPTRL MNVVTEQIYDKLFEIDRHTHRVIPSLAETFSVSDDGKEITLNLRROVAFHKTPWFTPTRI $* * * * * * * * * * * * * * * * * * * * * * * * * * * * * * * * * * * * * * * * * * * * * * * * * * * * * * * * * * * * *$

FNAEDVVFSLNRMIGNVEELPALDFNEDSKEQFQQNQRYAYHFKANLAHYPYFESVALKK FNAEDVVFSLNHMIGNVEELPALDFNEDSKEQFQQNQRYAYHFKANLAHYPYFESVALKK FNAEDVVF SLNHMIGNVEELPALDFNEDSKEQFQQNQRYAYHFKANLAHYPYFESVALKK FNAEDVVF SLNHMIGNVEELPALDFNEDSKEQFQQNQRYAYHFKANLAHYPYFESVALKK FNAEDVVF SLNHMIGNVEELPALDFNEDSKEOFOQNORYAYHFKANLAHYPYFESVALKK FNAEDVVF SLNRMIGNVEELPALDFNEDSKEQFQQNQRYAYHFKANLAHYPYFESVALKK FNAEDVVF SLNRMIGNVEELPALDFNEDSKEQFQQNQRYAYHFKANLAHYPYFESVALKK FNAEDVVFSLNRMIGNVEELPALDFNEDSKEQFQQNQRYAYHFKANLAHYPYFESVALKK FNAEDVVFSLNRMIGNVEELPALDFNEDSKEQFQQNQRYAYHFKANLAHYPYFESVALKK $* * * * * * * * * * *: * * * * * * * * * * * * * * * * * * * * * * * * * * * * * * * * * * * * * * * * * * * * * * * *$

KIAKISAPNEYTVKIHLVAPDNSVLAHLASQYAVILSKEYALLLNADENLAQLDLLPVGT KIAKISAPNEYTVKIHLVAPDNSVLAHLASOYAVILSKEYALLLNADENLAOLDLLPVGT KIAKISAPNEYTVKI HLVAPDNSVLAHLASQYAVILSKEYALLLNADENLAQLDLLPVGT KIAKISAPNEYTVKIHLVAPDNSVLAHLASQYAVILSKEYALLLNADENLAQLDLLPVGT KIAKISAPNEYTVKIHLVAPDNSVLAHLASQYAVILSKEYALLLNADENLAQLDLLPVGT KIVKISAPNEYTVKIHLVAPDNSVLAHLASOYAVILSKEYALLLNADENLAQLDLLPVGT KIVKISAPNEYTVKIHLVAPDNSVLAHLASQYAVILSKEYALLLNADENLAQLDLLPVGT KIVKISAPNEYTVKIHLVAPDNSVLAHLASOYAVILSKEYALLLNADENLAQLDLLPVGT KIVKISAPNEYTVKIHLVAPDNSVLAHLASOYAVILSKEYALLLNADENLAOLDLLPVGT $* * . * * * * * * * * * * * * * * * * * * * * * * * * * * * * * * * * * * * * * * * * * * * * * * * * * * * * * * * * *$ GVYQLSDYIQNEYVRLKPNPVYWGEKAKINNVVVDFSSNSTGRMAKYLNQECDIVAQPEP GVYQLSDY IQNEYVRLKPNPVYWGEKAKINNVVVDFSSNSTGRMAKYLNQECDIVAQPEP 
6644

HMC 46

HMC 56

33921

CIP5 42

DMC6 4

DMC 111

$35000 \mathrm{HP}$

82-029362

6644

HMC 46

HMC 56

33921

CIP 542

DMC 64

DMC 111

$35000 \mathrm{HP}$

82-029362

6644

HMC 46

HMC 56

33921

CIP 542

DMC 64

DMC 111

$35000 \mathrm{HP}$

82-029362

6644

HMC 46

HMC 56

33921

CIP 542

DMC 64

DMC 111

$35000 \mathrm{HP}$

82-029362

6644

HMC 46

HMC 56

33921

CIP 542

DMC6 4

DMC 111

$35000 \mathrm{HP}$

82-029362

6644

HMC 46

HMC 56

33921

CIP 542

DMC 64

DMC 111

GVYQLSDYIQNEYVRLKPNPVYWGEKAKINNVVVDFSSNSTGRMAKYLNQECDIVAQPEP GVYQLSDYIQNEYVRLKPNPVYWGEKAKINNVVVDFSSNSTGRMAKYLNQECDIVAQPEP GVYQLSDYIQNEYVRLKPNPVYWGEKAK INNVVVDF SSNSTGRMAKYLNQECDIVAQPEP GVYQLSDYIQNEYVRLKPNPVYWGEKAKINNVVVDFSSNSTGRMAKYLNQECDIVAQPEP GVYQLSDYIQNEYVRLKPNPVYWGEKAKINNVVVDFSSNSTGRMAKYLNQECDIVAQPEP GVYQLSDYIQNEYVRLKPNPVYWGEKAKINNVVVDFSSNSTGRMAKYLNQECDIVAQPEP GVYQLSDYIQNEYVRLKPNPVYWGEKAKINNVVVDFSSNSTGRMAKYLNQECDIVAQPEP $\star * * * * * * * * * * * * * * * * * * * * * * * * * * * * * * * * * * * * * * * * * * * * * * * * * * * * * * * * * * *$

SQRRVISSYEIVESPGANLAFLAFNMQKEKMQDIAFRRQIAQA INRERLVKALFYGSAEV SORRVISSYEIVESPGANLAFLAFNMOKEKMQDIAFRRQIAQAINRERLVKALFYGSAEV SQRRVISSYEIVESPGANLAFLAFNMQKEKMQDIAFRRQIAQAINRERLVKALFYGSAEV SQRRVISSYEIVESPGANLAFLAFNMQKEKMQDIAFRRQIAQA INRERLVKALFYGSAEV SQRRVISSYEIVESPGANLAFLAFNMOKEKMODIAFRRQIAQA INRERLVKALFYGSAEV SQRRVISSYEIVESPGANLAFLAFNMQKEKMQDIAFRRQIAQA INRERLVKALFYGSAEV SQRRVISSYEIVESPGANLAFLAFNMQKEKMQDIAFRRQIAQAINRERLVKALFYGSAEV SQRRVISSYEIVESPGANLAFLAFNMQKEKMQDIAFRRQIAQA INRERLVKALFYGSAEV SQRRVISSYEIVESPGANLAFLAFNMQKEKMQDIAFRRQIAQAINRERLVKALFYGSAEV

$* * * * * * * * * * * * * * * * * * * * * * * * * * * * * * * * * * * * * * * * * * * * * * * * * * * * * * * * * * * *$

ADNVLPSALFAQKNPAAYPYKAPQPRAKNAKLDRLIFWVLDESRVYNLHPLKMAEMIRND ADNVLPSALFAQKNPAAYPYKAPQPRAKNAKLDRLIFWVLDESRVYNLHPLKMAEMIRND ADNVLPSALFAQKNPAAYPYKAPQPRAKNAKLDRLIFWVLDESRVYNLHPLKMAEMIRND ADNVLPSALFAQKNPAAYPYKAPQPRAKNAKLDRLIFWVLDESRVYNLHPLKMAEMIRND ADNVLPSALFAQKNPAAYPYKAPQPRAKNAKLDRLIFWVLDESRVYNLHPLKMAEMIRND ADNVLPSALFAQKNPAAYPYKAPQPRAKNAKLDRLIFWVLDESRVYNLHPLKMAEMIRND ADNVLPSALFAQKNPAAYPYKAPQPRAKNAKLDRLIFWVLDESRVYNLHPLKMAEMIRND ADNVLPSALFAQKNPAAYPYKAPQPRAKNAKLDRLIFWVLDESRVYNLHPLKMAEMIRND ADNVLPSALFAQKNPAAYPYKAPQPRAKNAKLDRLIFWVLDESRVYNLHPLKMAEMIRND $* * * * * * * * * * * * * * * * * * * * * * * * * * * * * * * * * * * * * * * * * * * * * * * * * * * * * * * * * * * *$

LKKINIDVI IRPVSRAKVVQLAAAGKADYDLILTGWLANNLDPNAFLSPILSCRTQNKVT LKKINIDVI IRPVSRAKVVQLAAAGKADYDLILTGWLANNLDPNAFLSP ILSCRTQNKVT LKKINIDVI IRPVSRAKVVQLAAAGKADYDLILTGWLANNLDPNAFLSPILSCRTQNKVT LKKINIDVI IRPVSRAKVVQLAAAGKADYDLILTGWLANNLDPNAFLSP ILSCRTQNKVT LKKINIDVI IRPVSRAKVVQLAAAGKADYDLILTGWLANNLDPNAFLSP ILSCRTQNKVT LKKINIDAI IRPVSRAKVVQLAAAGKADYDLILTGWLANNLDPNAFLSPILSCRTQNKVT LKKINIDAI IRPVSRAKVVQLAAAGKADYDLILTGWLANNLDPNAFLSP ILSCRTQNKVT LKK IN I DA I IRPVSRAKVVQLAAAGKADYDL ILTGWLANNLDPNAFLSP ILSCRTQNKVT LKKINIDAI IRPVSRAKVVQLAAAGKADYDLILTGWLANNLDPNAFLSPILSCRTQNKVT $* * * * * * * . * * * * * * * * * * * * * * * * * * * * * * * * * * * * * * * * * * * * * * * * * * * * * * * * * * * *$

NLANWCHQQFDEWLEIAKANQVPYVRNMIYKQTQALLEEQLPILPLLHAQRSLFVNQKIK NLANWCHQQFDEWLE IAKANQVPYVRNMIYKQTQALLEEQLP ILPLLHAQRSLFVNQKIK NLANWCHQQFDEWLE IAKANQVPYVRNMIYKQTQALLEEQLP ILPLLHAQRSLFVNQKIK NLANWCHQQFDEWLEIAKANQVPYVRNMIYKQTQALLEEQLP ILPLLHAQRSLFVNQKIK NLANWCHQQFDEWLE IAKANQVPYVRNMIYKQTQALLEEQLP ILPLLHAQRSLFVNQKIK NLANWCHQQFDEWLE IAKANQVPYVRNMIDKQTQALLEEQLP ILPLLHAQRSLFVNQKIK NLANWCHQQFDEWLEIAKANQVPYVRNMIDKQTQALLEEQLP ILPLLHAQRSLFVNQKIK NLANWCHQQFDEWLE IAKANQVPYVRNMIDKQTQALLEEQLP ILPLLHAQRSLFVNQK IK NLANWCHQQFDEWLE IAKANQVPYVRNMIDKQTQALLEEQLP ILPLLHAQRSLFVNQKIK $* * * * * * * * * * * * * * * * * * * * * * * * * * * * * * * * * * * * * * * * * * * * * * * * * * * * * * * * * * *$

NAH IEPFGQVRLSELTLHQE * NAH IEPFGOVRLSELTLHOE * NAH IEPFGQVRLSELTLHQE * NAH IEPFGQVRLSELTLHQE* NAH IEPFGQVRLSELTLHQE * NAH IEPFGQVRLSELTLHQE * NAH IEPFGQVRLSELTLHQE* NAH IEPFGQVRLSELTLHQE * NAH IEPFGQVRLSELTLHQE* $* * * * * * * * * * * * * * * * * * * * *$

Note: The SapA sequence of NZS1, NZS2, NZS3, NZS4, and NZV1 were identical to that of $35000 \mathrm{HP}$.

\section{9) SapB (HD1231)}

$35000 \mathrm{HP}$

33921

CIP 542
MLFAF IRRLFLSLITLIILTLIGYNILLRDPLNHFMDLYGIOAYFSYVMGLLHGDFGISY MLFAFIRRLFLSLITLIILTLIGYNILLRDPLNHFMDLYGIQAYFSYVMGLLHGDFGISY MLFAF IRRLFLSLITLIILTLIGYNILLRDPLNHFMDLYGIQAYFSYVMGLLHGDFGISY 
DMC64 MLFAFIRRLFLSLITLIILTLIGYNILLRDPLNHFMDLYGIOAYFSYVMGLLHGDFGISY DMC111 MLFAFIRRLFLSLITLIILTLIGYNILLRDPLNHFMDLYGIQAYFSYVMGLLHGDFGISY $\star * * * * * * * * * * * * * * * * * * * * * * * * * * * * * * * * * * * * * * * * * * * * * * * * * * * * * * * * * * *$

$35000 \mathrm{HP}$ SNGDPIANQILNVFPATISLCFAALFVSVIIGIPLGFVAASFRYNVVGKLLAIVSAFSLA 33921 SNGDPIANQILNVFPATISLCFAALFVSVIIGIPLGFVAASFRDNVVGKLLAIVSAFSLA CIP542 SNGDPIANQILNVFPATISLCFAALFVSVIIGIPLGFVAASFRDNVVGKLLAIVSAFSLA DMC64 SNGDPIANQILNVFPATISLCFAALFVSVIIGIPLGFVAASFRDNVVGKLLAIVSAFSLA DMC111 SNGDPIANQILNVFPATISLCFAALFVSVIIGIPLGFVAASFRDNVVGKLLAIVSAFSLA $* * * * * * * * * * * * * * * * * * * * * * * * * * * * * * * * * * * * * * * * * * * * * * * * * * * * * * * * * * *$

$35000 \mathrm{HP}$ IPVFWLAIMALYYAASNDWQIAAVGELHPIYEISLVTGFRLLDIFLADSPYKLKMMOSVL 33921 IPVFWLAIMALYYAASNDWQIAAVGELHPIYEISLVTGFRLLDIFLADSPYKLKMMQSVL CIP542 IPVFWLAIMALYYAASNDWOIAAVGELHPIYEISLVTGFRLLDIFLADSPYKLKMMOSVL DMC64 IPVFWLAIMALYYAASNDWQIAAVGELHPIYEISLVTGFRLLDIFLADSPYKLKMMOSVL DMC111 IPVFWLAIMALYYAASNDWQIAAVGELHPIYEISLVTGFRLLDIFLADSPYKLKMMOSVL $* * * * * * * * * * * * * * * * * * * * * * * * * * * * * * * * * * * * * * * * * * * * * * * * * * * * * * * * * * * *$

35000HP HHLALPTLILALPATLEVIRFTRORAEYVMKONYIKVARTRGWSPYKIWLKHILRNTLPA 33921

CIP5 42

DMC 64

DMC 111 HHLALPTLILALPATLEVIRFTRQRAEYVMKQNYIKVARTRGWSPYKIWLKHILRNTLPA HHLALPTLILALPATLEVIRFTRORAEYVMKQNYIKVARTRGWSPYKIWLKH ILRNTLPA HHLALPTLILALPATLEVIRFTRQRAEYVMKQNYIKVARTRGWSPYKIWLKHILRNTLPA HHLALPTLILALPATLEVIRFTRQRAEYVMKQNYIKVARTRGWSPYKIWLKHILRNTLPA $* * * * * * * * * * * * * * * * * * * * * * * * * * * * * * * * * * * * * * * * * * * * * * * * * * * * * * * * * * * *$

$35000 \mathrm{HP}$ LIPMIARNLTLVFAFAMLVENIFSWGGIGLWLINALAIODYNAISAGVVAIGLFVLGVDI 33921

CIP5 42

DMC64

DMC 111 LIPMIARNLTLVFAFAMLVENIF SWGGIGLWLINALAIQDYNAISAGVVAIDLFVLGVDI LIPMIARNLTLVFAFAMLVENIFSWGGIGLWLINALAIODYNAISAGVVAIDLFVLGVDI LIPMIARNLTLVFAFAMLVENIFSWGGIGLWLINALAIQDYNAISAGVVAIDLFVLGVDI LIPMIARNLTLVFAFAMLVENIFSWGGIGLWLINALAIQDYNAISAGVVAIDLFVLGVDI

$35000 \mathrm{HP} \quad$ LVRLVTTLLDPSOKKDWYVK *

33921 LVRLVTTLLDPSQKKDWYVK *

CIP54 2 LVRLVTTLLDPSQKKDWYVK*

DMC6 4 LVRLVTTLLDPSQKKDWYVK*

DMC111 LVRLVTTLLDPSQKKDWYVK *

$* * * * * * * * * * * * * * * * * * * * *$

Note: The SapB sequence of NZS1, NZS2, NZS3, NZS4, NZV1, 82-029362, 6644, HD183, HMC46, and HMC56 were identical to that of $35000 \mathrm{HP}$.

\section{0) SapC (HD1232)}

$35000 \mathrm{HP}$

NZS1

NZS2

NZS3

NZS 4

NZV1

82-029362

6644

HD 183

HMC 46

HMC 56

33921

CIP5 42

DMC 64

DMC 111

$35000 \mathrm{HP}$

NZS 1

NZS2

NZS 3

NZS 4

NZV1

82-029362

6644

HD 183

HMC 46

HMC 56
MLNREEPEOFROSDYAKOFWLELRODKVALASLYFFLILLFLTFAGELIAPYOVNTOFVG MLNREEPEQFRQSDYAKQFWLELRQDKVALASLYFFLILLFLTFAGELIAPYQVNTQFVG MLNREEPEOFRQSDYAKQFWLELRODKVALASLYFFLILLFLTFAGELIAPYQVNTQFVG MLNREEPEQFRQSDYAKQFWLELRODKVALASLYFFLILLFLTFAGELIAPYQVNTOFVG MLNREEPEQFRQSDYAKQFWLELRQDKVALASLYFFLILLFLTFAGELIAPYQVNTQFVG MLNREEPEQFRQSDYAKQFWLELRQDKVALASLYFFLILLFLTFAGELIAPYQVNTQFVG MLNREEPEQFRQSDYAKQFWLELRQDKVALASLYFFLILLFLTFAGELIAPYQVNTQFVG MLNREEPEQFRQSDYAKQFWLELRQDKVALASLYFFLILLFLTFAGELIAPYQVNTQFVG MLNREEPEQFRQSDYAKQFWLELRQDKVALASLYFFLILLFLTFAGELIAPYQVNTQFVG MLNREEPEOFROSDYAKOFWLELRODKVALASLYFFLILLFLTFAGELIAPYOVNTOFVG MLNREEPEQFRQSDYAKQFWLELRQDKVALASLYFFLILLFLTFAGELIAPYQVNTQFVG MLNREEPEQFRQSDYAKQFWLELRQDKVALASLYFFLILLFLTFAGELIAPYQVNTQFVG MLNREEPEQFRQSDYAKOFWLELRQDKVALASLYFFLILLFLTFAGELIAPYQVNTQFVG MLNREEPEQFRQSDYAKQFWLELRQDKVALASLYFFLILLFLTFAGELIAPYQVNTQFVG MLNREEPEOFROSDYAKOFWLELRODKVALASLYFFLILLFLTFAGELIAPYQVNTOFVG $* * * * * * * * * * * * * * * * * * * * * * * * * * * * * * * * * * * * * * * * * * * * * * * * * * * * * * * * * * * *$

FELLPPSWDDYGQISHFFGTDDLGRDIFSRILAGFYYTVGAALLISFAIAIIGGVIGVLA FELLPPSWDDYGQISHFFGTDDLGRDIF SRILAGFYYTVGAALLISFAIAIIGGVIGVLA FELLPPSWDDYGQISHFFGTDDLGRDIFSRILAGFYYTVGAALLISFAIAI IGGVIGVLA FELLPPSWDDYGQISHFFGTDDLGRDIFSRILAGFYYTVGAALLISFAIAI IGGVIGVLA FELLPPSWDDYGQISHFFGTDDLGRDIFSRILAGFYYTVGAALLISFAIAIIGGVIGVLA FELLPPSWDDYGQISHFFGTDDLGRDIFSRILAGFYYTVGAALLISFAIAI IGGVIGVLA FELLPPSWDDYGQISHFFGTDDLGRDIF SRILAGFYYTVGAALLISFAIAIIGGVIGVLA FELLPPSWDDYGQISHFFGTDDLGRDIFSRILAGFYYTVGAALLISFAIAI IGGVIGVLA FELLPPSWDDYGQISHFFGTDDLGRDIF SRILAGFYYTVGAALLISFAIAI IGGVIGVLA FELLPPSWDDYGOISHFFGTDDLGRDIFSRILAGFYYTVGAALLISFAIAIIGGVIGVLA FELLPPSWDDYGQISHFFGTDDLGRDIFSRILAGFYYTVGAALLISFAIAI IGGVIGVLA 
33921

CIP5 42

DMC 64

DMC 111

$35000 \mathrm{HP}$

NZS 1

NZS2

NZS 3

NZS 4

NZV1

82-029362

6644

HD 183

HMC 46

HMC 56

33921

CIP5 42

DMC 64

DMC 111

$35000 \mathrm{HP}$

NZS1

NZS2

NZS 3

NZS 4

NZV1

82-029362

6644

HD 183

HMC 46

HMC 56

33921

CIP5 42

DMC 64

DMC 111

$35000 \mathrm{HP}$

NZS 1

NZS2

NZS 3

NZS 4

NZV1

82-029362

6644

HD 183

HMC 46

HMC 56

33921

CIP 542

DMC 64

DMC 111
FELLPPSWGDYGQISYFFGTDDLGRDIFSRILAGFYYTVGAALLISFAIAIIGGVIGVLA FELLPPSWGDYGQISYFFGTDDLGRDIFSRILAGFYYTVGAALLISFAIAI IGGVIGVLA FELLPPSWGDYGQISYFFGTDDLGRDIF SRILAGFYYTVGAALLISFAIAI IGGVIGVLA FELLPPSWGDYGQISYFFGTDDLGRDIFSRILAGFYYTVGAALLISFAIAI IGGVIGVLA $* * * * * * * * * * * * * *: * * * * * * * * * * * * * * * * * * * * * * * * * * * * * * * * * * * * * * * * * * * *$

GTSRKAISFLGHLFDTFLF IPTLI IAII IATLMEASLINAMLAIFLAMLPHF IHKIYQAT GTSRKA ISFLGHLFDTFLF IPTLI IAI I IATLMEASLINAMLA IFLAMLPHF I HKIYQAT GTSRKA ISFLGHLFDTFLF IPTLI IAI I IATLMEASL INAMLA IFLAMLPHF I HKIYOAT GTSRKAISFLGHLFDTFLF IPTLI IAI I IATLMEASL INAMLA I FLAMLPHF I HKIYQAT GTSRKA ISFLGHLFDTFLF IPTLI I I I I ATLMEASL INAMLA I FLAMLPHF I HKIYOAT GTSRKAISFLGHLFDTFLF IPTLI I I I I ATLMEASL INAMLA I FLAMLPHF I HK I YQAT GTSRKAISFLGHLFDTFLF IPTLI IAI I IATLMEASLINAMLA I FLAMLPHF I HKIYQAT GTSRKAISFLGHLFDTFLF IPTLI IAI I IATLMEASLINAMLA I FLAMLPHF I HKIYQAT GTSRKAISFLGHLFDTFLF IPTLI IAI I IATLMEASL INAMLA I FLAMLPHF I HK I YQAT GTSRKA ISFLGHLFDTFLF IPTLI IAI I IATLMEASL INAMLA IFLAMLPHF I HK I YOAT GTSRKA ISFLGHLFDTFLF IPTLI IAI I IATLMEASL INAMLA IFLAMLPHF I HKIYQAT GTSRKA ISFLGHLFDTFLF IPTLI IAI I I ATLMEASL INAMLA IFLAMLPHF I HKIYQAT GTSRKAISFLGHLFDTFLF IPTLI IAI I IATLMEASLINAMLA I FLAMLPHF I HK IYQAT GTSRKAISFLGHLFDTFLF IPTLI IAI I IATLMEASLINAMLA I FLAMLPHF I HKIYQAT GTSRKA ISFLGHLFDTFLF IPTLI I I I IATLMEASLINAMLA I FLAMLPHF I HKI YQAT $* * * * * * * * * * * * * * * * * * * * * * * * * * * * * * * * * * * * * * * * * * * * * * * * * * * * * * * * * * * *$

EQOLKREYVITLRLDGISRWOLI KEVVLPNLTALAVKEMTH IC I IAVLDINALSF IGLGA EQQLKREYVITLRLDGISRWQLIKEVVLPNLTALAVKEMTH ICIIAVLDINALSF IGLGA EQQLKREYVITLRLDGISRWQLIKEVVLPNLTALAVKEMTH ICI IAVLDINALSF IGLGA EQQLKREYVITLRLDG ISRWQL I KEVVLPNLTALAVKEMTH ICI IAVLDINALSF IGLGA EOOLKREYVITLRLDG ISRWOL I KEVVLPNLTALAVKEMTH ICI IAVLDINALSF IGLGA EQQLKREYVITLRLDGISRWQL I KEVVLPNLTALAVKEMTH ICI IAVLDINALSF IGLGA EQQLKREYVITLRLDGISRWQLIKEVVLPNLTALAVKEMTH ICI IAVLDINALSF IGLGA EOOLKREYVITLRLDGISRWOLI KEVVLPNLTALAVKEMTH IC I IAVLDINALSF IGLGA EQQLKREYVITLRLDGISRWQL I KEVVLPNLTALAVKEMTH IC I IAVLDINALSF IGLGA EQQLKREYVITLRLDG ISRWOL I KEVVLPNLTALAVKEMTH ICI IAVLDINALSF I GLGA EQQLKREYVITLRLDG ISRWQLIKEVVLPNLTALAVKEMTH ICI IAVLDINALSF IGLGA EQQLKREYVITLRLDG ISRWQLI KEVVLPNLTALAVKEMTH IC I IAVLDINALSF IGLGA EQQLKREYVITLRLDGISRWQL I KEVVLPNLTALAVKEMTH ICI IAVLDINALSF IGLGA EQQLKREYVITLRLDGISRWQLIKEVVLPNLTALAVKEMTH ICIIAVLDINALSF IGLGA EQOLKREYVITLRLDGISRWOLI KEVVLPNLTALAVKEMTH ICI IAVLDINALSF IGLGA $* * * * * * * * * * * * * * * * * * * * * * * * * * * * * * * * * * * * * * * * * * * * * * * * * * * * * * * * * * * *$

QSPMPEWGVMIKDSIELIYIAPWTVILPGIITILVILIISMLGNSISRVLEKHRY* QSPMPEWGVMIKDS IELIY IAPWTVILPGI ITILVILI ISMLGNS ISRVLEKHRY* OSPMPEWGVMIKDSIELIYIAPWTVILPGIITILVILI ISMLGNSISRVLEKHRY* QSPMPEWGVMIKDSIELIYIAPWTVILPGIITILVILI ISMLGNSISRVLEKHRY* OSPMPEWGVMIKDS IELIY IAPWTVILPGI ITILVILI ISMLGNS I SRVLEKHRY* QSPMPEWGVMI KDSIELIYIAPWTVILPGIITILVILIISMLGNSISRVLEKHRY * QSPMPEWGVMIKDS IELIYIAPWTVILPGI ITILVILI ISMLGNS ISRVLEKHRY* OSPMPEWGVMIKDS IELIYIAPWTVILPGIITILVILI ISMLGNS I SRVLEKHRY* QSPMPEWGVMIKDSIELIYIAPWTVILPGIITILVILI ISMLGNS ISRVLEKHRY* OSPMPEWGVMIKDS IELIYIAPWTVILPGI ITILVILI ISMLGNS I SRVLEKHRY * QSPMPEWGVMI KDS IELIYIAPWTVILPGI ITILVILI ISMLGNS I SRVLEKHRY * QSPMPEWGVMIKDS IELIY IAPWTVILPGI ITILVILI ISMLGNS ISRVLEKHRY* QSPMPEWGVMIKDS IELIYIAPWTVILPGIITILVILI ISMLGNS ISRVLEKHRY * QSPMPEWGVMIKDS IELIY IAPWTVILPGI ITILVILI ISMLGNS ISRVLEKHRY * OSPMPEWGVMIKDSIELIYIAPWTVILPGIITILVILIISMLGNS ISRVLEKHRY* $* * * * * * * * * * * * * * * * * * * * * * * * * * * * * * * * * * * * * * * * * * * * * * * * * * * * * * * *$

\section{1) SpoT (HD1924)}

$35000 \mathrm{HP}$

33921

CIP5 42

DMC 64

DMC111

$35000 \mathrm{HP}$

33921

CIP5 42

DMC6 4

DMC 111
LHLFEPLHSI IOTYLPTDKIEWIORAFVVARDAHEGQTRSSGEPY ITHPVAVATI IAEMK LHLFEPLHSI IQTYLPTDKIEWIQRAFVVARDAHEGQTRSSGEPY ITHPVAVATI IAEMK LHLFEPLHSI IQTYLPTDKIEWIORAFVVARDAHEGQTRSSGEPY ITHPVAVATI IAEMK LHLFEPLHSI IQTYLPTDKIEWIQRAFVVARDAHEGQTRSSGEPYITHPVAVATI IAEMK LHLFEPLHSI IQTYLPTDKIEWIQRAFVVARDAHEGQTRSSGEPYITHPVAVATI IAEMK $* * * * * * * * * * * * * * * * * * * * * * * * * * * * * * * * * * * * * * * * * * * * * * * * * * * * * * * * * * * * *$

LDHEAIMAALLHDVIEDTPYTEEELAAEFGSNVAKIVQGVSKLDKLKFRTRQEAQVENFR LDHEAIMAALLHDVIEDTPYTEEELAAEFGSNVAKIVQGVSKLDKLKFRTRQEAQVENFR LDHEAIMAALLHDVIEDTPYTEEELAAEFGSNVAKIVQGVSKLDKLKFRTRQEAQVENFR LDHEAIMAALLHDVIEDTPYTEEELAAEFGSNVAKIVQGVSKLDKLKFRTRQEAQVENFR LDHEAIMAALLHDVIEDTPYTEEELAAEFGSNVAKIVQGVSKLDKLKFRTRQEAOVENFR 


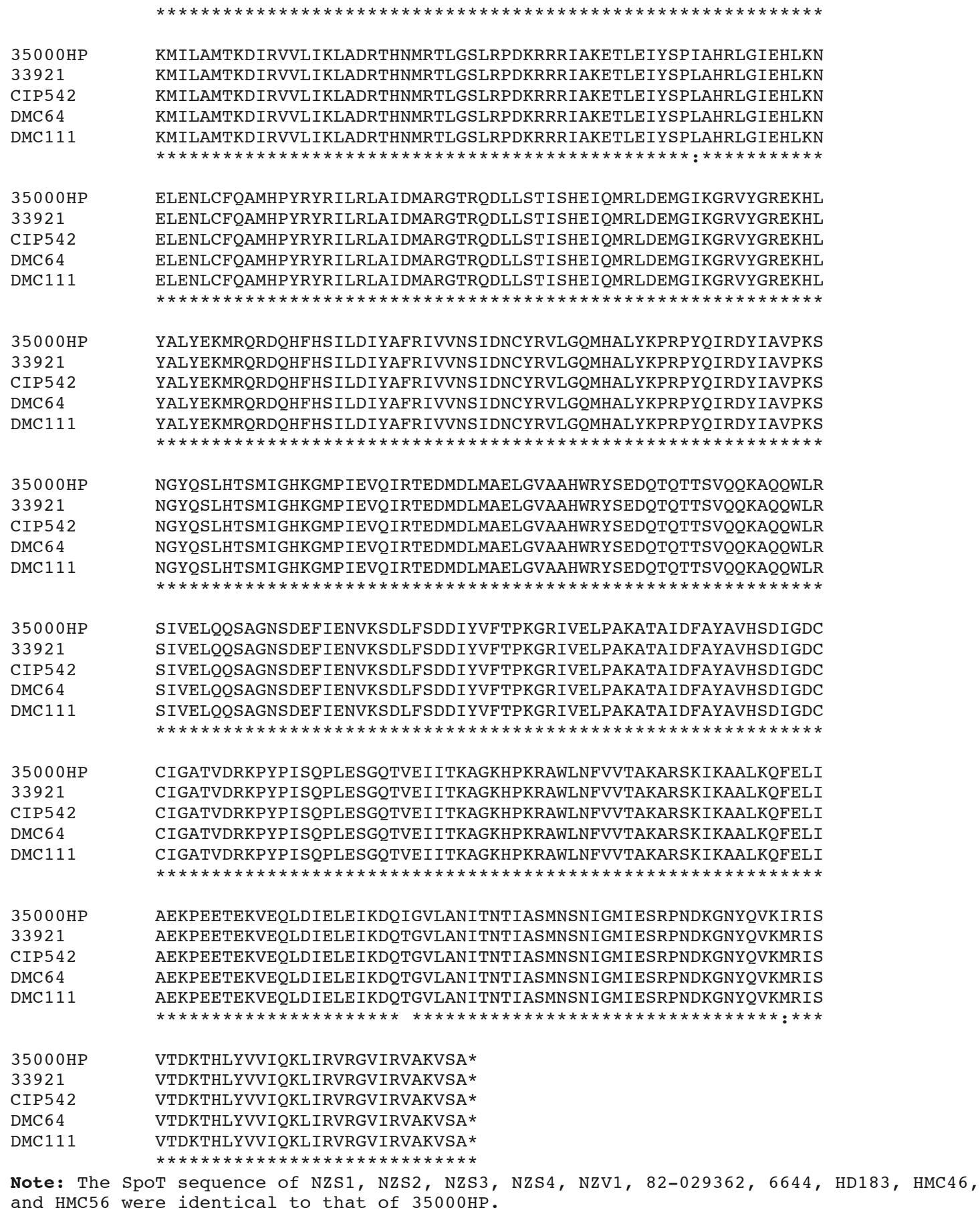

\section{2) TadA (HD1304)}

$35000 \mathrm{HP}$

$82-029362$

6644

HD183

HMC 46

HMC56

33921

CIP5 42

DMC64
MLTKDQQVFFRNALLSNLNVDTLDEIENERSKLVTELTQSLYRVANTNNIYITPYDATDM MLTKDQQVFFRNALLSNLNVDTLDEIENERSKLVTELTQSLYRVANTNNIYITPYDATDM MLTKDQQVFFRNALLSNLNVDTLDEIENERSKLVTELTQSLYRVANTNNIYITPYDATDM MLTKDQQVFFRNALLSNLNVDTLDEIENERSKLVTELTQSLYRVANTNNIYITPYDATDM MLTKDQQVFFRNALLSNLNVDTLDEIENERSKLVTELTQSLYRVANTNNIYITPYDATDM MLTKDQQVFFRNALLSNLNVDTLDEIENERSKLVTELTQSLYRVANTNNIYITPYDATDM MLTKDQQVFFRNALLSNLNVDTLDEIENERSKLVTELTQSLYRVANTNNIYITPYDATDM MLTKDQQVFFRNALLSNLNVDTLDEIENERSKLVTELTQSLYRVANTNNIYITPYDATDM MLTKDQQVFFRNALLSNLNVDTLDEIENERSKLVTELTQSLYRVANTNNIYITPYDATDM 
$35000 \mathrm{HP}$

82-029362

6644

HD 183

HMC 46

HMC 56

33921

CIP 542

DMC 64

DMC 111

$35000 \mathrm{HP}$

82-029362

6644

HD 183

HMC 46

HMC 56

33921

CIP 542

DMC 64

DMC 111

$35000 \mathrm{HP}$

82-029362

6644

HD 183

HMC 46

HMC 56

33921

CIP5 42

DMC 64

DMC 111

$35000 \mathrm{HP}$

82-029362

6644

HD 183

HMC 46

HMC 56

33921

CIP 542

DMC6 4

DMC 111

$35000 \mathrm{HP}$

82-029362

6644

HD 183

HMC 46

HMC 56

33921

CIP54 2

DMC6 4

DMC 111

$35000 \mathrm{HP}$

82-029362

6644

HD 183

HMC 46

HMC 56

33921

CIP 542

DMC6 4

DMC 111
AEIVADEIGGYGPIRELMEDDTVNDILVNGPDNIWIERAGVLEKTNKTF INNEOLTDIAK AEIVADEIGGYGP IRELMEDDTVNDILVNGPDN IWIERAGVLEKTNKTF INNEQLTDIAK AEIVADEIGGYGP IRELMEDDTVNDILVNGPDN IWIERAGVLEKTNKTF INNEQLTDIAK AEIVADEIGGYGPIRELMEDDTVNDILVNGPDNIWIERAGVLEKTNKTF INNEQLTDIAK AEIVADE IGGYGPIRELMEDDTVNDILVNGPDN IWIERAGVLEKTNKTF INNEQLTDIAK AEIVADE IGGYGP IRELMEDDTVNDILVNGPDNIWIERAGVLEKTNKTF INNEOLTDIAK AEIVADE IGGYGP IRELMEDDTVNDILVNGPDN IWVERAGVLEKTNKTF INNEQLTDIAK AEIVADEIGGYGPIRELMEDDTVNDILVNGPDN IWVERAGVLEKTNKTF INNEQLTDIAK AEIVADE I GGYGP IRELMEDDTVNDILVNGPDNIWVERAGVLEKTNKTF INNEQLTDIAK AEIVADE IGGYGP IRELMEDDTVNDILVNGPDN IWVERAGVLEKTNKTF INNEQLTDIAK $* * * * * * * * * * * * * * * * * * * * * * * * * * * * * * * * * * *: * * * * * * * * * * * * * * * * * * * * * * * *$

RLVARVGRRIDEGMPLVDSRLPDGSRLNVVIOPIALDGTS ISIRKFSKSKKSLQELVNFG RLVARVGRRIDEGMPLVDSRLPDGSRLNVVIQPIALDGTS ISIRKF SKSKKSLQELVNFG RLVARVGRRIDEGMPLVDSRLPDGSRLNVVIQPIALDGTS ISIRKF SKSKKSLQELVNFG RLVARVGRRIDEGMPLVDSRLPDGSRLNVVIOPIALDGTS ISIRKF SKSKKSLQELVNFG RLVARVGRRIDEGMPLVDSRLPDGSRLNVVIQPIALDGTS ISIRKF SKSKKSLQELVNFG RLVARVGRRIDEGMPLVDSRLPDGSRLNVVIQPIALDGTS ISIRKFSKSKKSLQELVNFG RLVARVGRRIDESMPLVDSRLPDGSRLNVVIQPIALDGTS ISIRKF SKSKKSLQELVNFG RLVARVGRRIDESMPLVDSRLPDGSRLNVVIQPIALDGTS ISIRKF SKSKKSLQELVNFG RLVARVGRRIDESMPLVDSRLPDGSRLNVVIQPIALDGTS ISIRKF SKSKKSLQELVNFG RLVARVGRRIDESMPLVDSRLPDGSRLNVVIQPIALDGTSISIRKFSKSKKSLQELVNFG $* * * * * * * * * * * * . * * * * * * * * * * * * * * * * * * * * * * * * * * * * * * * * * * * * * * * * * * * * * * *$

SMTLDMANFLIIAARSRVNI IVSGGTGSGKTTLLNALSSY ISPTERVLTLEDTAELRLEO SMTLDMANFLI IAARSRVNI IVSGGTGSGKTTLLNALSSY ISPTERVLTLEDTAELRLEQ SMTLDMANFLIIAARSRVNI IVSGGTGSGKTTLLNALSSY ISPTERVLTLEDTAELRLEQ SMTLDMANFLIIAARSRVNI IVSGGTGSGKTTLLNALSSY ISPTERVLTLEDTAELRLEQ SMTLDMANFLIIAARSRVNI IVSGGTGSGKTTLLNALSSY ISPTERVLTLEDTAELRLEQ SMTLDMANFLIIAARSRVNI IVSGGTGSGKTTLLNALSSY ISPTERVLTLEDTAELRLEQ SMTLDMANFLIIAARSRVNI IVSGGTGSGKTTLLNALSSY ISPTERVLTLEDTAELRLEQ SMTLDMANFLI IAARSRVNI IVSGGTGSGKTTLLNALSSY ISPTERVLTLEDTAELRLEO SMTLDMANFLI IAARSRVNI IVSGGTGSGKTTLLNALSSY ISPTERVLTLEDTAELRLEQ SMTLDMANFLI IAARSRVNI IVSGGTGSGKTTLLNALSSY ISPTERVLTLEDTAELRLEQ

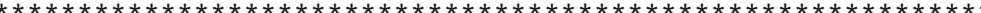

PHVVRLETRLAGVERTGEITMODLVINALRMRPERI IVGECRGAEAFOMLOAMNTGHDGS PHVVRLETRLAGVERTGEITMQDLVINALRMRPERI IVGECRGAEAFQMLQAMNTGHDGS PHVVRLETRLAGVERTGEITMQDLVINALRMRPERIIVGECRGAEAFQMLQAMNTGHDGS PHVVRLETRLAGVERTGEITMODLVINALRMRPERIIVGECRGAEAFOMLOAMNTGHDGS PHVVRLETRLAGVERTGE I TMQDLVINALRMRPERI IVGECRGAEAFOMLQAMNTGHDGS PHVVRLETRLAGVERTGEITMQDLVINALRMRPERI IVGECRGAEAFQMLQAMNTGHDGS PHVVRLETRLAGVERTGEITMODLVINALRMRPERI IVGECRGAEAFQMLQAMNTGHDGS PHVVRLETRLAGVERTGE ITMODLVINALRMRPERI IVGECRGAEAFOMLQAMNTGHDGS PHVVRLETRLAGVERTGE ITMODLVINALRMRPERI IVGECRGAEAFOMLQAMNTGHDGS PHVVRLETRLAGVERTGEITMQDLVINALRMRPERI IVGECRGAEAFQMLQAMNTGHDGS

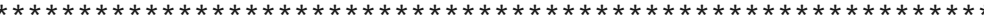

MSTLHANTPRDATARLESMVMMSNASLPLEAIRRNIASAVNI I IQASRLNDGSRKVMNIT MSTLHANTPRDATARLESMVMMSNASLPLEAIRRNIASAVNI I IQASRLNDGSRKVMNIT MSTLHANTPRDATARLESMVMMSNASLPLEAIRRNIASAVNI I IQASRLNDGSRKVMNIT MSTLHANTPRDATARLESMVMMSNASLPLEAIRRNIASAVNI I IQASRLNDGSRKVMNIT MSTLHANTPRDATARLESMVMMSNASLPLEAIRRNIASAVNIIIQASRLNDGSRKVMNIT MSTLHANTPRDATARLESMVMMSNASLPLEAIRRNIASAVNI I IOASRLNDGSRKVMNIT MSTLHANTPRDATARLESMVMMSNASLPLEAIRRNIASAVNI I IQASRLNDGSRKVMNIT MSTLHANTPRDATARLESMVMMSNASLPLEAIRRNIASAVNI I IOASRLNDGSRKVMNIT MSTLHANTPRDATARLESMVMMSNASLPLEAIRRNIASAVNII IOASRLNDGSRKVMNIT MSTLHANTPRDATARLESMVMMSNASLPLEAIRRNIASAVNIIIQASRLNDGSRKVMNIT $* * * * * * * * * * * * * * * * * * * * * * * * * * * * * * * * * * * * * * * * * * * * * * * * * * * * * * * * * * * *$

EVMGMENGQIVLQDIFSFEASQHRDENNKI IGKFVNHGLLTRSAVYQNAQVFNLTGELQN EVMGMENGQIVLQDIFSFEASQHRDENNKI IGKFVNHGLLTRSAVYQNAQVFNLTGELQN EVMGMENGQIVLQDIF SFEASQHRDENNKI I GKFVNHGLLTRSAVYQNAQVFNLTGELQN EVMGMENGQIVLQDIFSFEASQHRDENNKI IGKFVNHGLLTRSAVYQNAQVFNLTGELQN EVMGMENGQIVLQDIF SFEASOHRDENNKI I GKFVNHGLLTRSAVYQNAQVFNLTGELQN EVMGMENGOIVLODIF SFEASOHRDENNKI IGKFVNHGLLTRSAVYONAOVFNLTGELON EVMGMENGQIVLQDIF SFEPSQHRDENNKI I GKFVNHGLLTRSAVYQNAQVFNLTGELQN EVMGMENGQIVLQDIF SFEPSQHRDENNKI I GKFVNHGLLTRSAVYQNAOVFNLTGELQN EVMGMENGQIVLQDIFSFEPSQHRDENNKI IGKFVNHGLLTRSAVYQNAQVFNLTGELQN EVMGMENGQIVLQDIF SFEPSQHRDENNKI IGKFVNHGLLTRSAVYQNAQVFNLTGELQN 
Note: The TadA sequence of NZS1, NZS2, NZS3, NZS4, and NZV1 were identical to that of $35000 \mathrm{HP}$.

\section{3) WecA (HD1844)}

$35000 \mathrm{HP}$ MRRLLKKFKSRYLYAILHICYIILSFRNNFLMWLTF IAVF IVSFASLILMRPVAEKIGLI 33921 MRRLLKKFESRYLYAILHICYIILSFRNNFLMWLTFIAVF IVSFASLILMRPVAEKIGLI CIP542 MRRLLKKFESRYLYAILHICYIILSFRNNFLMWLTFIAVF IVSFASLILMRPVAEKIGLI DMC64 MRRLLKKFESRYLYAILHICYIILSFRNNFLMWLTFIAVFIVSFASLILMRPVAEKIGLI DMC111 MRRLLKKFESRYLYAILHICYIILSFRNNFLMWLTFIAVF IVSFASLILMRPVAEKIGLI $* * * * * * * *: * * * * * * * * * * * * * * * * * * * * * * * * * * * * * * * * * * * * * * * * * * * * * * * * * * * *$

$35000 \mathrm{HP}$ DKPNYRKRHQGLIPLIGGIALFLGNLTFYFIEWQDMRLPWLYLTAVTVLLVIGLLDDRFD 33921

CIP5 42

DMC 64

DMC 111 DKSNYRKRHQGLIPLIGGIALFLGNLTFYF IEWQDMRLPWLYLTAVTVLLVIGLLDDRFD DKSNYRKRHOGLIPLIGGIALFLGNLTFYF IEWODMRLPWLYLTAVTVLLVIGLLDDRFD DKSNYRKRHQGLIPLIGGIALFLGNLTFYFIEWQDMRLPWLYLTAVTVLLVIGLLDDRFD DKSNYRKRHQGLIPLIGGIALFLGNLTFYF IEWQDMRLPWLYLTAVTVLLVIGLLDDRFD

$35000 \mathrm{HP}$ VSPFLRIGLQAGLAGLMIYHGLSLESLGQVIAPFS IKLGILGTVFTILITIGVINAFNMV 33921 VSPFLRIGLQAGLAGLMIYHGLSLESLGQVIAPFSIKLGILGTVFTILITIGVINAFNMV CIP542 VSPFLRIGLQAGLAGLMIYHGLSLESLGQVIAPFSIKLGILGTVFTILITIGVINAFNMV DMC64 VSPFLRIGLQAGLAGLMIYHGLSLESLGQVIAPFSIKLGILGTVFTILITIGVINAFNMV DMC111 VSPFLRIGLQAGLAGLMIYHGLSLESLGQVIAPFS IKLGILGTVFTILITIGVINAFNMV

$35000 \mathrm{HP}$ DGIDGLLAGLSSASFAGIGVLMWLDEOYSLAYWCFALIVVLIPYAMFNLSLLGAKWKVFM 33921 DGIDGLLAGLSSASFAGIGVLMWLDEQYSLAYWCFALIVVLIPYAMFNLSLLGPKWKVFM CIP542 DGIDGLLAGLSSASFAGIGVLMWLDEQYSLAYWCFALIVVLIPYAMFNLSLLGPKWKVFM DMC64 DGIDGLLAGLSSASFAGIGVLMWLDEQYSLAYWCFALIVVLIPYAMFNLSLLGPKWKVFM DMC111 DGIDGLLAGLSSASFAGIGVLMWLDEQYSLAYWCFALIVVLIPYAMFNLSLLGPKWKVFM $* * * * * * * * * * * * * * * * * * * * * * * * * * * * * * * * * * * * * * * * * * * * * * * * * * * * * * * * * * * *$

$35000 \mathrm{HP}$ GDSGSTLIGFTIIWILLLSTQGQGHAISPITGLWLIAVPLIDMVAVVLRRLKKGKSPFRP 33921

CIP 542

DMC 64

DMC 111 GDSGSTLIGFTIIWILLLSTQGQGHAISPITGLWLIAVPLIDMVAVVLRRLKKGKSPFRP GDSGSTLIGFTI IWILLLSTQGOGHAISPITGLWLIAVPLIDMVAVVLRRLKKGKSPFRP GDSGSTLIGFTIIWILLLSTQGQGHAISPITGLWLIAVPLIDMVAVVLRRLKKGKSPFRP GDSGSTLIGFTIIWILLLSTQGQGHAISPITGLWLIAVPLIDMVAVVLRRLKKGKSPFRP $\star * * * * * * * * * * * * * * * * * * * * * * * * * * * * * * * * * * * * * * * * * * * * * * * * * * * * * * * * * * *$

$35000 \mathrm{HP}$

33921

CIP5 42

DMC 64

DMC 111

DRLHLHHLMMRAGLTSRQALAVITLGATICSMIGVFGEYYYWNQWAMTAGF IALFF IYAY DCLHLHHLMMRAGLTSRQALAVITLGATICSMIGVLGEYYYWNQWAMTAGF IALFF IYAY DCLHLHHLMMRAGLTSRQALAVITLGATICSMIGVLGEYYYWNQWAMTAGF IALFF I YAY DCLHLHHLMMRAGLTSRQALAVITLGATICSMIGVLGEYYYWNQWAMTAGF IALFF IYAY DCLHLHHLMMRAGLTSRQALAVITLGATICSMIGVLGEYYYWNQWAMTAGF IALFF I YAY $* * * * * * * * * * * * * * * * * * * * * * * * * * * * * * * * * *: * * * * * * * * * * * * * * * * * * * * * * * *$

$35000 \mathrm{HP}$ SITHAWRMTRFIRRMKRREKRKQHA*

33921 SITHAWRMTRFVRRMKRREKRKOHA*

CIP54 2 SITHAWRMTRFVRRMKRREKRKOHA*

DMC64 SITHAWRMTRFVRRMKRREKRKOHA*

DMC111 SITHAWRMTRFVRRMKRREKRKQHA* $* * * * * * * * * * *: * * * * * * * * * * * * * *$

Note: The WecA sequence of NZS1, NZS2, NZS3, NZS4, NZV1, 82-029362, 6644, HD183, HMC46, and HMC5 6 were identical to that of $35000 \mathrm{HP}$. 